\title{
Estimating 1970-99 Average Annual Groundwater Recharge in Wisconsin Using Streamflow Data
}

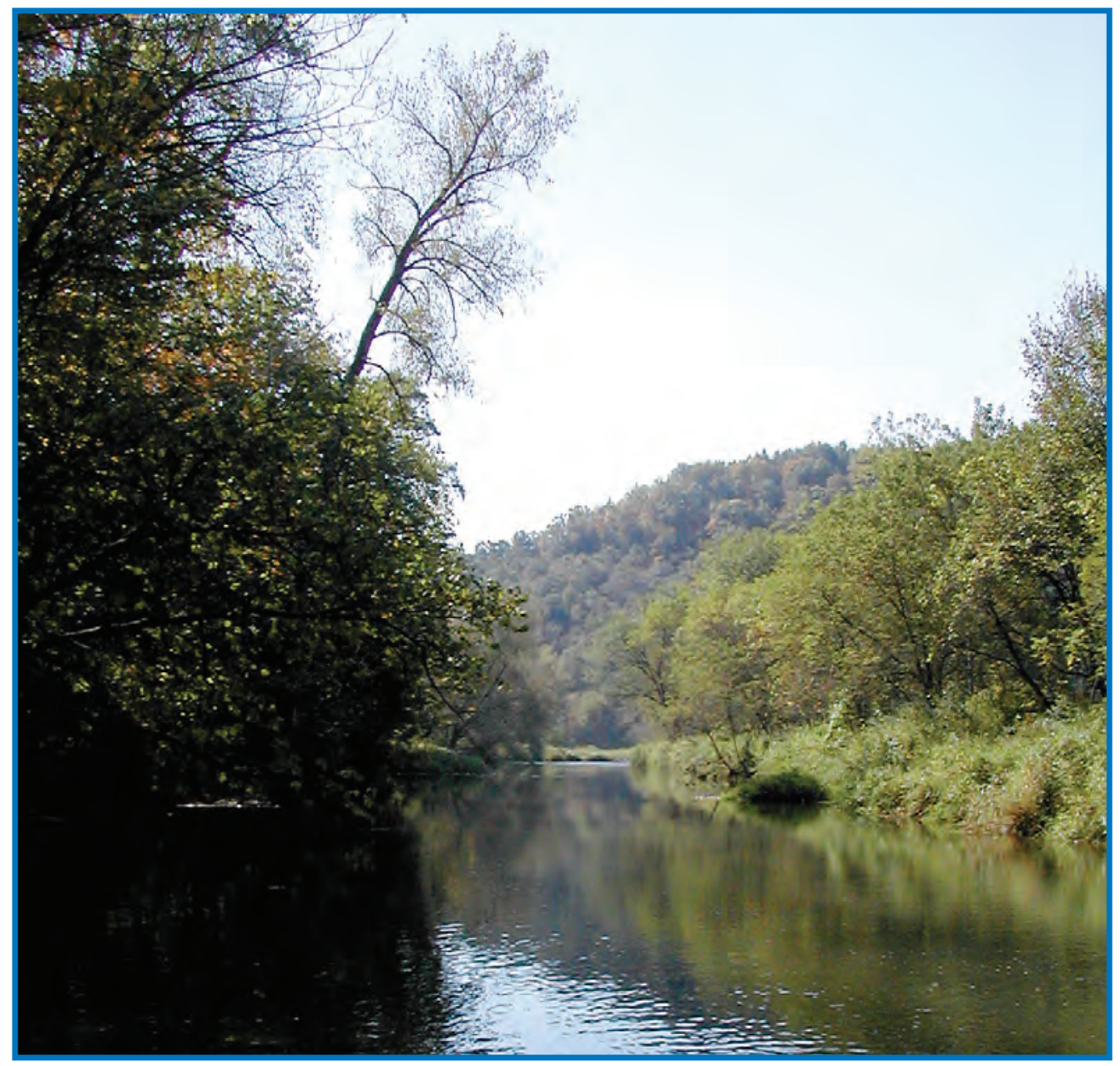

Open-File Report 2009-1210 



\section{Estimating 1970-99 Average Annual Groundwater Recharge in Wisconsin Using Streamflow Data}

By Warren A. Gebert, John F. Walker, and James L. Kennedy

Open-File Report 2009-1210 


\section{U.S. Department of the Interior \\ KEN SALAZAR, Secretary \\ U.S. Geological Survey \\ Marcia K. McNutt, Director}

U.S. Geological Survey, Reston, Virginia: 2011

For more information on the USGS - the Federal source for science about the Earth, its natural and living resources, natural hazards, and the environment, visit http://www.usgs.gov or call 1-888-ASK-USGS

For an overview of USGS information products, including maps, imagery, and publications, visit http://www.usgs.gov/pubprod

To order this and other USGS information products, visit http://store.usgs.gov

Any use of trade, product, or firm names is for descriptive purposes only and does not imply endorsement by the U.S. Government.

Although this report is in the public domain, permission must be secured from the individual copyright owners to reproduce any copyrighted materials contained within this report.

Suggested citation:

Gebert, W.A., Walker, J.F., and Kennedy, J.L., 2011, Estimating 1970-99 average annual recharge in Wisconsin using streamflow data: Reston, Va., U.S. Geological Survey Open-File Report 2009-1210, 14 p. plus appendixes.

Cover: West Fork of the Kickapoo River, Wisconsin. Photograph by Herbert S. Garn. 


\section{Contents}

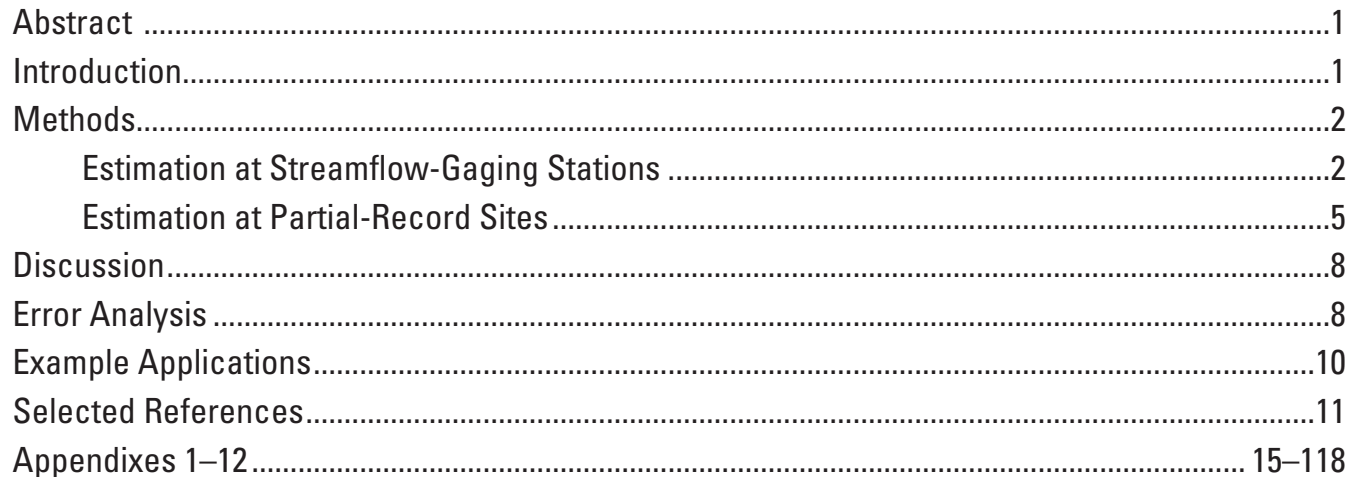

$A$, Locations of streamflow-gaging stations and spatial distribution of average annual recharge; $B$, locations of miscellaneous and partial-record sites and spatial distribution of estimated average annual recharge; $C$, average annual base flow and corresponding recharge, 1970-99, for streamflow-gaging stations; and $D$, average annual base flow and corresponding recharge, 1970-99, for partial-record stations.

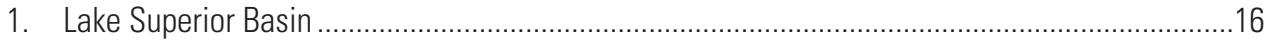

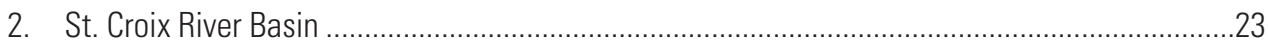

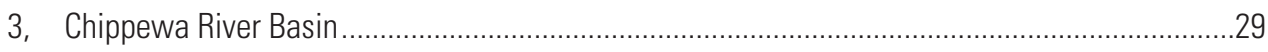

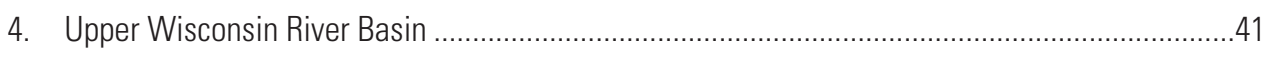

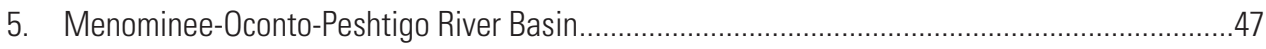

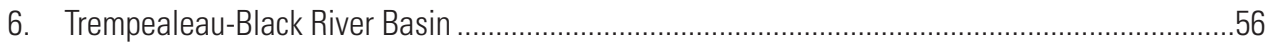

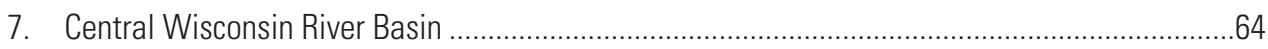

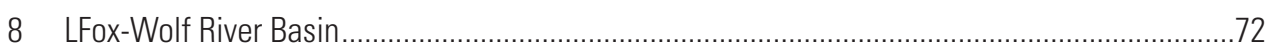

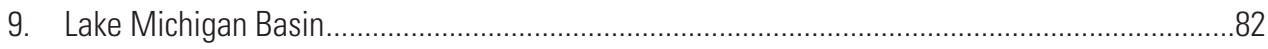

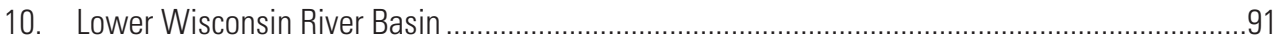

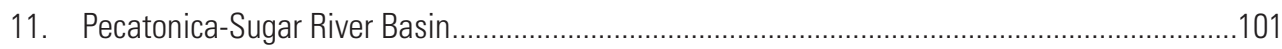

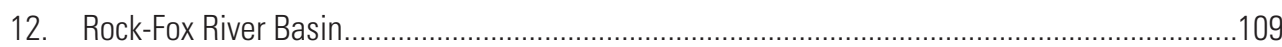

\section{Figures}

1-2. Maps showing:

1. Spatial distribution of base-flow index, 1970-99, at streamflow-gaging stations in Wisconsin.

2. Spatial distribution of average annual recharge, 1970-99, at streamflowgaging stations in Wisconsin.

3. Graph showing example relation line and estimation technique for partialrecord site Elk Creek at Elk Creek, Wisconsin.

4. Map showing spatial distribution of average annual recharge, 1970-99, at partial-record sites in Wisconsin.....

5-6. Graphs showing:

5. Comparison of annual base flow estimated using the relation-line approach (estimated) and the Base Flow Index approach (actual) at selected streamflow-gaging stations 
6. Comparison of annual base flow estimated using the statewide-regression approach (estimated) and the Base Flow Index approach (actual) at selected streamflow-gaging stations

\section{Tables}

1. Comparison of baseflow estimates from graphical and Base Flow Index methods (Institute of Hydrology, 1980a, 1980b) for six streamflow-gaging stations in Wisconsin for water year 1967

2. Comparison of recharge estimates from calibrated ground-water flow models and techniques described in this report

\section{Conversion Factors}

\section{Inch/Pound to SI}

\begin{tabular}{|c|c|c|}
\hline Multiply & By & To obtain \\
\hline \multicolumn{3}{|c|}{ Length } \\
\hline inch (in.) & 2.54 & centimeter $(\mathrm{cm})$ \\
\hline \multicolumn{3}{|c|}{ Area } \\
\hline square mile $\left(\mathrm{mi}^{2}\right)$ & 259.0 & hectare (ha) \\
\hline square mile $\left(\mathrm{mi}^{2}\right)$ & 2.590 & square kilometer $\left(\mathrm{km}^{2}\right)$ \\
\hline \multicolumn{3}{|c|}{ Flow rate } \\
\hline cubic foot per second $\left(\mathrm{ft}^{3} / \mathrm{s}\right)$ & 0.02832 & cubic meter per second $\left(\mathrm{m}^{3} / \mathrm{s}\right)$ \\
\hline $\begin{array}{l}\text { cubic foot per second per square mile } \\
{\left[\left(\mathrm{ft}^{3} / \mathrm{s}\right) / \mathrm{mi}^{2}\right]}\end{array}$ & 0.01093 & $\begin{array}{l}\text { cubic meter per second per square } \\
\text { kilometer }\left[\left(\mathrm{m}^{3} / \mathrm{s}\right) / \mathrm{km}^{2}\right]\end{array}$ \\
\hline inch per year (in/yr) & 2.54 & centimeter per year $(\mathrm{cm} / \mathrm{yr})$ \\
\hline
\end{tabular}




\title{
Estimating 1970-99 Average Annual Groundwater Recharge in Wisconsin Using Streamflow Data
}

\author{
By Warren A. Gebert, John F. Walker, and James L. Kennedy
}

\section{Abstract}

Average annual recharge in Wisconsin for the period 1970-99 was estimated using streamflow data from U.S. Geological Survey continuous-record streamflow-gaging stations and partial-record sites. Partial-record sites have discharge measurements collected during low-flow conditions. The average annual base flow of a stream divided by the drainage area is a good approximation of the recharge rate; therefore, once average annual base flow is determined recharge can be calculated. Estimates of recharge for nearly 72 percent of the surface area of the State are provided. The results illustrate substantial spatial variability of recharge across the State, ranging from less than 1 inch to more than 12 inches per year. The average basin size for partial-record sites ( 50 square miles) was less than the average basin size for the gaging stations (305 square miles). Including results for smaller basins reveals a spatial variability that otherwise would be smoothed out using only estimates for larger basins. An error analysis indicates that the techniques used provide base flow estimates with standard errors ranging from 5.4 to 14 percent.

\section{Introduction}

The purpose of this report is to present the methods used and results of a study to determine the average annual recharge using base flow derived from U.S. Geological Survey (USGS) streamflow sites in Wisconsin. The groundwater component of streamflow is important since it is indicative of the sustained flow of the stream during dry periods, is generally of better quality, and is cooler in temperature. All three of these characteristics are important to the health of aquatic life in a stream. If recharge to the aquifers is to be preserved or enhanced, it is important to know the present partitioning of total streamflow into base flow and stormflow. An estimate of groundwater recharge also is important for the calibration and verification of groundwater models that are being used to investigate many of the water-supply and contamination problems faced by communities relying on groundwater as a source of drinking water (Cherkauer, 2004).

The U.S. Geological Survey operates numerous continuous-record streamflow-gaging stations, which can be used to provide estimates of average annual base flow. In addition to the continuous-record stations, a substantial number of partial-record sites were operated during 1964-1990 throughout the State for various low-flow studies; these sites generally contain 1 to 15 discharge measurements made during low-flow conditions. The collection of partial-record sites can be used for additional estimates of average annual base flow. While the average drainage area at the 123 gaging stations used in the statewide analysis is $305 \mathrm{mi}^{2}$, the average drainage area of the 1,495 partial-record sites is around $50 \mathrm{mi}^{2}$. The use of data at this scale provides estimates of average annual base flow from headwater streams as opposed to the higher order streams represented by the 123 gaging stations.

In a general sense, base flow separation provides an estimate of groundwater discharge to the stream. Recharge, on the other hand, is comprised of infiltrated water that reaches the water table. For average values over relatively long periods in many hydrologic settings, recharge can be represented by groundwater discharge (Rutledge, 2007). A reasonable estimate of contributing area allows conversion of the groundwater discharge in cubic feet per second to a recharge rate in inches. 


\section{Methods}

Estimation of recharge using base flow and drainage area requires reasonable estimates of the average base flow in a stream. Groundwater recharge processes and associated base flow function at different scales (Scanlon and others, 2002). To cover a range of scales, estimates are provided using both continuous-record streamflow-gaging stations and partialrecord sites.

\section{Estimation at Streamflow-Gaging Stations}

The recorded daily discharge at the streamflow-gaging stations was separated into base flow and stormflow using the Base Flow Index (BFI) automated hydrograph separation application (Institute of Hydrology, 1980 a, b). The BFI application divides the water year into $\mathrm{N}$-day increments and the minimum flow for each N-day period is identified, where $\mathrm{N}$ is a user-specified duration in days. Minimum flows are then compared to adjacent minimum flows to determine turning points on the base-flow hydrograph. Minimum flows that are less than a fixed proportion (f) of adjacent minimum flows are designated as turning points, and a straight line is established between turning points. The area below this line is an estimate of the volume of base flow. The ratio of volume of base flow for the water year to the total runoff volume for the water year is defined to be the "base-flow index." In contrast to manual procedures, the BFI application can handle large amounts of data and is objective. The application produces a table of base flow, total streamflow, and base-flow index for each water year, as well as summary statistics. A FORTRAN program that implements the Institute of Hydrology (1980a, b) method was written for studies of base-flow trends in the Oklahoma Panhandle (Wahl and Wahl, 1995) and has been modified since its usage during these studies to increase its usefulness and provide flexibility.

The BFI application was applied to the streamflow at 123 continuous-record gaging stations to determine the average annual base flow and total streamflow for each station. Trend analysis in previous studies (Gebert and Krug, 1996; Gebert and others, 2007) found statistically significant trends in streamflow for the long-term period of record at many streamflow-gaging stations. However, only one station demonstrated a significant trend in streamflow for the 1970-99 period. Furthermore, the 1970-99 period represents recent conditions, thus it was selected for analysis in this study. Stations were selected on unregulated streams to provide uniform coverage throughout Wisconsin. A 5-day interval was used, and the turning point proportion ( $\mathrm{f}$ ) was set to 0.90 for all stations. The interval and turning point proportion were recommended values, and inspection of the results for a few of the stations verified that the values provided acceptable base-flow separations. The resulting values for base-flow index are presented in figure 1.

For stations with considerably less than 30 years of record during the 1970-99 period, a relation line was established between annual base-flow values at the station for the period available (correlated station) and corresponding annual base-flow values at a nearby streamflow-gaging station with a complete record (index station). The relation line was used to translate average annual base flow for the 1970-99 period at the index station to average annual base flow for the same period at the correlated station; this procedure is identified as an extended record. The estimated average annual base flow is then divided by the basin area to give an estimate of recharge, in inches, for the basin. The resulting annual recharge values for the 123 streamflow-gaging stations are reported in the Appendixes and shown in figure 2.

In some cases, there are two or more sites on the same stream segment. For these cases, the recharge for the intervening area was calculated by subtracting base flow for the next upstream gage from the base flow from the downstream gage and dividing by the difference in drainage areas. The adjusted values for the intervening areas are depicted on the maps in the main report and the Appendixes; the tables in the Appendixes report the estimated base flow and recharge for the entire area upstream of each site. 


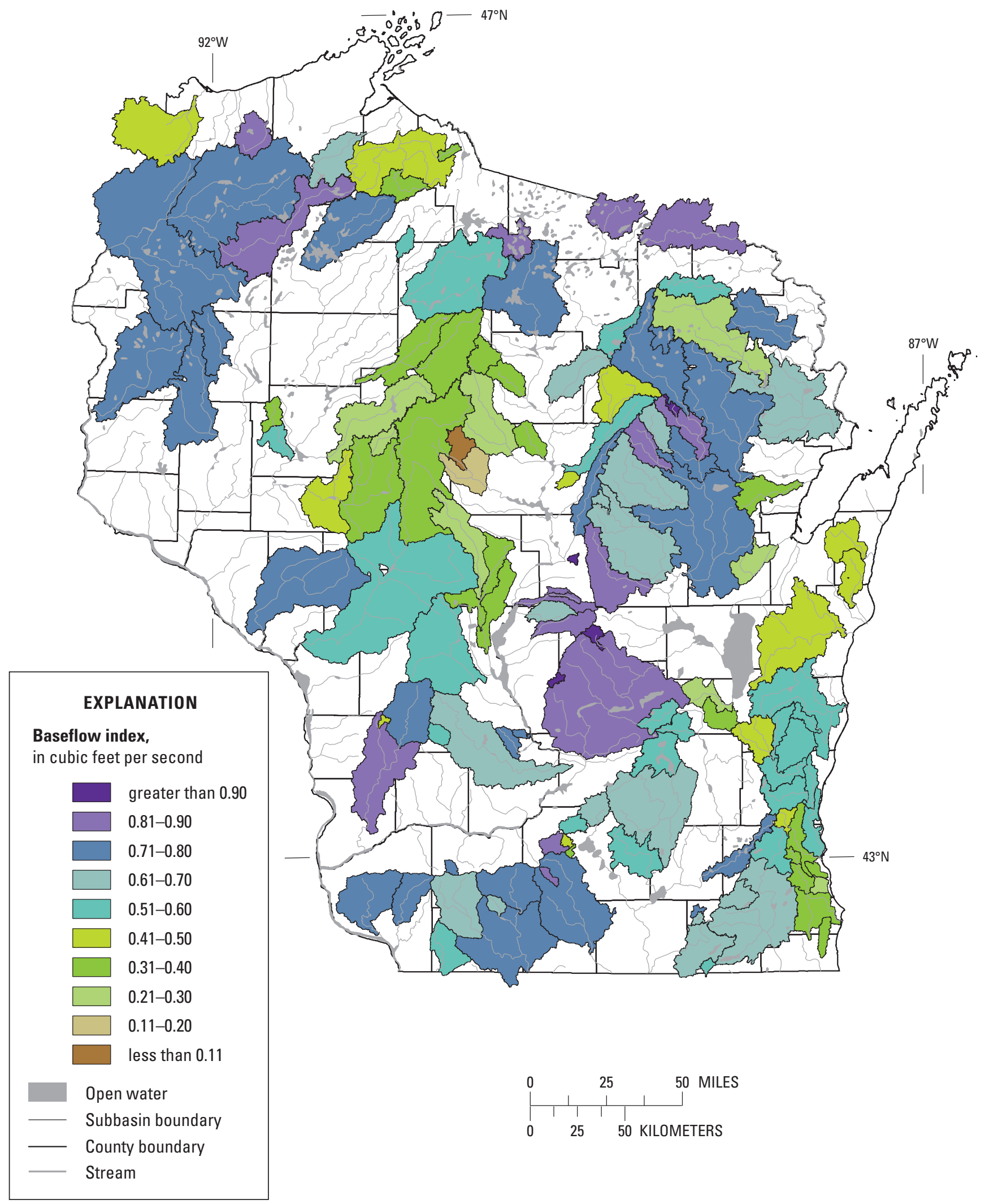

Figure 1. Spatial distribution of base-flow index, 1970-99, at streamflow-gaging stations in Wisconsin. 


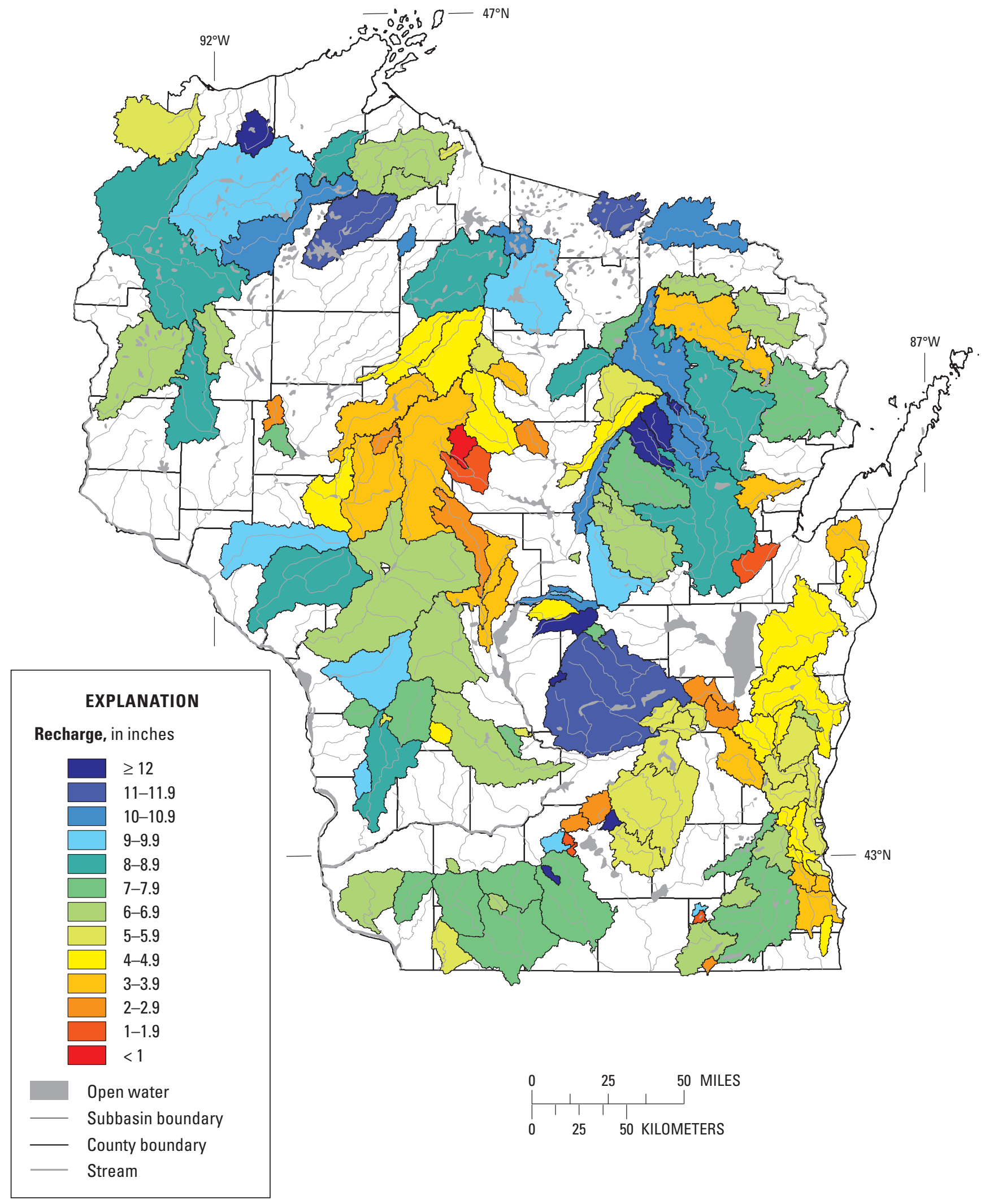

Figure 2. Spatial distribution of average annual recharge, 1970-99, at streamflow-gaging stations in Wisconsin. 


\section{Estimation at Partial-Record Sites}

Partial-record sites in Wisconsin were operated in conjunction with low-flow studies to allow estimates of low-flow statistics throughout Wisconsin. The low-flow studies were conducted for each of the 12 major river basins in the State. The approach described in the next two paragraphs will be referred to as the relation-line approach. During a low-flow study, 6 to 15 low-flow discharge measurements were made at selected partial-record sites. These measurements were then compared to one or more nearby streamflow-gaging stations to establish a relation between measured discharge and concurrent daily discharge at the gaging station. For each candidate streamflow-gaging station, the measurements were plotted against the concurrent daily discharges, and a best-fit relation was drawn by hand through the points. The station that provided the best relation with the measurements at the partial-record site, based on a visual inspection of the plot, was chosen as the index station for the site. For each partial-record site, estimates of average annual base flow can be obtained from the established relation line with the index station. An example of that method is shown for the partial-record site Elk Creek at Elk Creek (fig. 3). In this example, 10 discharge measurements made during low-flow conditions during 1969-80 are plotted against the recorded daily discharge on the day of the measurement at the Trempealeau River at Dodge index station to determine the relation line. The average annual base flow of $402 \mathrm{ft}^{3} / \mathrm{s}$ at the index station, determined by the baseflow separation analysis described in the previous section, is projected through the relation line to provide an estimate of the average annual base flow of $50 \mathrm{ft}^{3} / \mathrm{s}$ at the Elk Creek at Elk Creek partial-record site.

In some cases the relation line provides an unreasonable estimate of annual base flow. This can be due to either a poorly defined relation line or a relation line with a steep slope that results in an unreasonably high value when the annual basefow value at the index station lies outside the range of values used to define the relation line. For those cases, the following statewide-regression equation (Gebert and others, 2007) was used to estimate the average annual base flow at the partialrecord site:

$$
Q_{b}=0.907 A^{1.02} B_{f}^{0.52}
$$

where $Q_{b}$ is the average annual base flow in cubic feet per second, $A$ is the drainage area in square miles, and $B_{f}$ is the base-flow factor, in cubic feet per second per square mile.

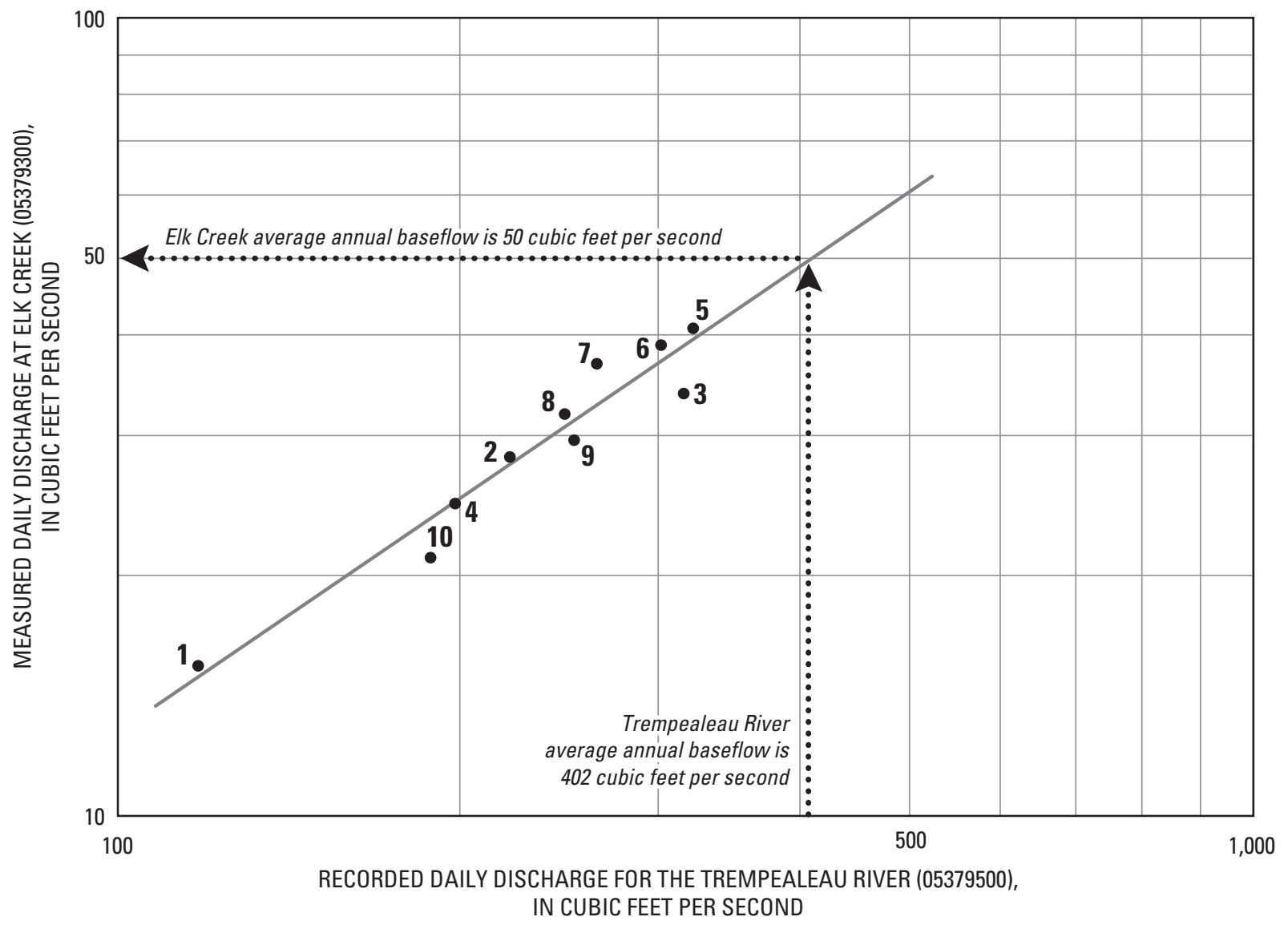

Figure 3. Example relation line and estimation technique for partial-record site Elk Creek at Elk Creek, Wisconsin. 
The base-flow factor is determined using a discharge measurement at the partial-record site and corresponding information at an index station:

$$
B_{f}=\left(\frac{Q_{m}}{Q_{r}^{*}}\right)\left(\frac{Q_{90}^{*}}{A}\right)
$$

where $B_{f}$ is the base-flow factor in cubic feet per second per square mile, $Q_{m}$ is the measured discharge at the site in cubic feet per second, $Q_{r}^{*}$ is the recorded daily discharge at the index station on the day the measurement was made in cubic feet per second, $Q_{90}^{*}$ is the 90 -percent flow-duration value at the index station in cubic feet per second, and $\mathrm{A}$ is the drainage area of the station in square miles. In cases where multiple measurements were made, the average of the individual base-flow factors was used.

During the course of the low-flow studies, 1 to 3 discharge measurements were made at additional sites throughout each river basin. The approach described in the next three paragraphs will be referred to as the statewide-regression approach. The miscellaneous-measurement sites were generally chosen on first- and second-order streams with relatively small drainage basins. Typically, a series of discharge measurements were made over a 1 to 5 day period when flow conditions were low. For these sites, the discharge measurements were used along with the statewide-regression equation, equation 1, to estimate average annual base flow.

Application of the statewide regression requires determination of the base-flow factor, which depends on measured discharge at the miscellaneous site and recorded daily discharge at an index station. The additional partial-record sites typically have 1 to 3 discharge measurements; therefore, it was not possible to compare the measurements to several gaging stations to determine the most appropriate index station. As a surrogate, proximity to partial-record sites with established index stations and knowledge of the regional hydrology were used to associate index stations with the additional sites.

Maps displaying the basin boundaries for the partialrecord sites with established index sites, the location of the additional partial-record sites, and the locations of the index stations were generated using Geographic Information System (GIS) techniques (Brenden and others, 2006). The basin boundaries of the partial-record sites with established index stations were shaded to indicate the index station used for each site. Index stations were assigned to each additional partial-record site based on the distribution of the index sites and knowledge of the local hydrology. Once the index station was established, the base-flow factor was determined using equation 2 , and the resulting average-annual base flow was determined using equation 1.

The results for the partial-record sites using the relationline and statewide-regression procedures are given in Appendixes 1-12 and displayed in figure 4. In some cases, there are two or more sites on the same stream segment. For these cases, recharge for the intervening area was calculated by subtracting base flow for the next upstream site from the base flow from the downstream site and dividing by the difference in drainage areas. The adjusted values for the intervening areas are depicted on figures 2 and 4 and the maps in the Appendixes; the tables in the Appendixes report the estimated base flow and recharge for the area upstream of each site. 


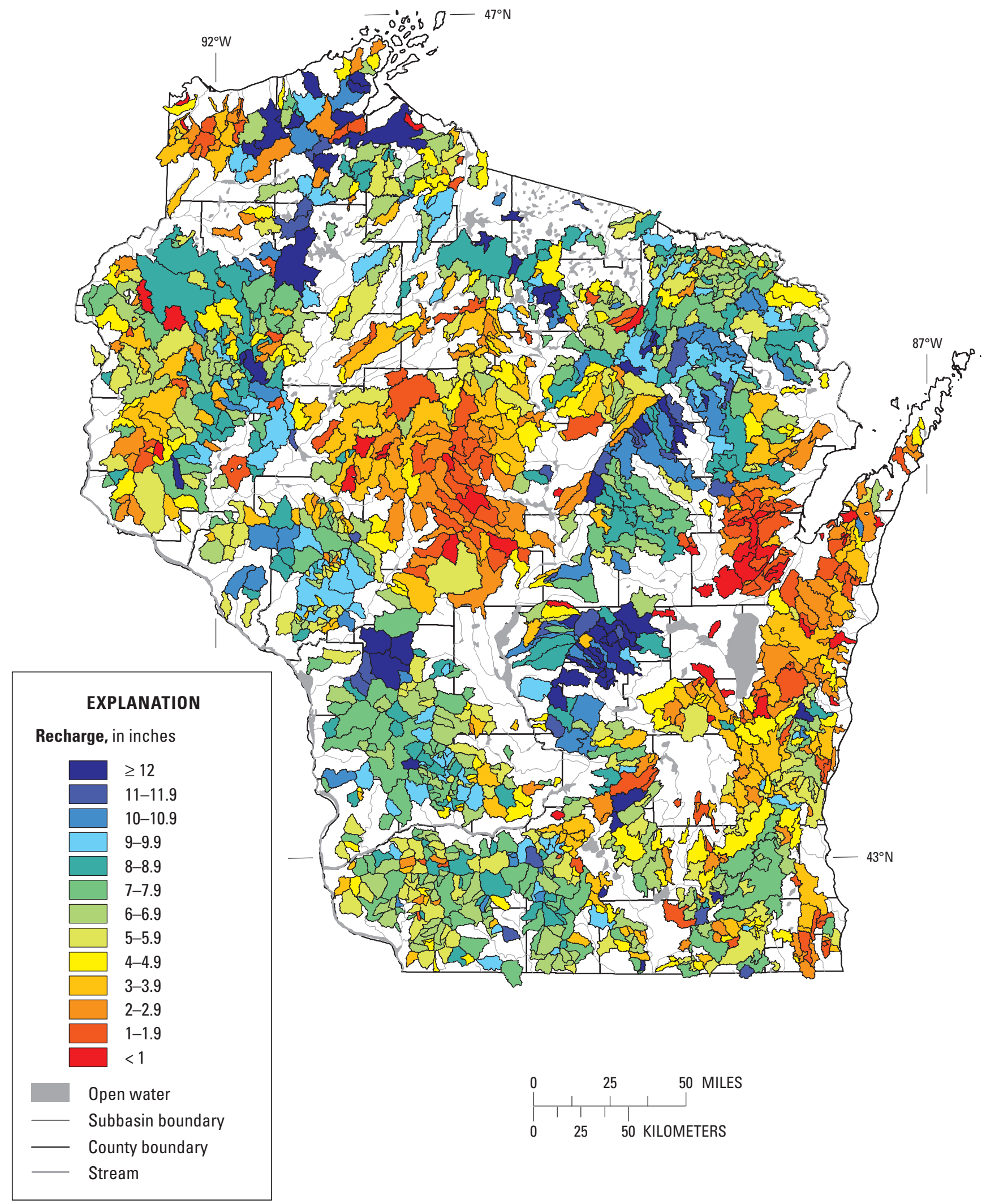

Figure 4. Spatial distribution of average annual recharge, 1970-99, at partial-record sites in Wisconsin. 


\section{Discussion}

The results for streamflow-gaging stations (fig. 2) and partial-record sites (fig. 4) illustrate a range of estimated recharge rates for Wisconsin-from less than 1 in. to more than $12 \mathrm{in.}$ The area covered by the two maps accounts for nearly 72 percent of the surface area of the State. Recharge in excess of 12 in. and less than 3 in. is potentially unrealistic given the magnitude of precipitation and evapotranspiration across Wisconsin. In some cases, the ground- and surface-water basin boundaries did not align very closely, resulting in excessively high or low values. Sites with values exceeding 12 in. and less than 3 in. were examined in detail to determine the likely cause of the extreme value; for cases where a drainage-area discrepancy was evident, the values in the Appendix tables are flagged accordingly.

The difference in drainage area scale for streamflow-gaging stations (average $305 \mathrm{mi}^{2}$ ) and partial-record sites (average $50 \mathrm{mi}^{2}$ ) can be used to illustrate the effect of basin scale on spatial variability of recharge. Since the value reported for a particular type of site represents an average over the entire drainage area, values for larger basins likely will be less variable than values for smaller areas because the larger areas will tend to integrate processes occurring at smaller scales. Thus, one would expect more variability for the partial-record sites (fig. 4) than the streamflow-gaging stations (fig. 2). This is particularly evident when comparing a value for a streamflowgaging station to the values for partial-record sites that are contained within the gaging station drainage boundary. For example, there are several streamflow-gaging stations in the northwest part of the State that have a number of partial-record sites nested within the subbasin boundary for each gaging station. The variability of the values for the smaller subbasins is appreciably higher than for the larger streamflow-gaging stations that contain the partial-record sites.

From the results shown in figures 2 and 4, general patterns in the average annual recharge can be seen in the State. The northern forested portion of the State contains some of the highest values, due in part to highly permeable sandy soils, a thick transmissive aquifer, and somewhat lower evapotranspiration. Fairly high values also are found in the central portion of the State (east of the Wisconsin River). This portion of the State, referred to as the Central Sand Plains (Martin, 1965; Dott and Attig, 2004), contains fairly thick, highly conductive sandy sediments and a transmissive aquifer. These basin properties, along with the area's flat terrain, facilitate the infiltration of water into the groundwater system rather than becoming stormflow or evapotranspiration. There is a fairly uniform distribution of recharge in the southwest portion of the State, often referred to as the Driftless Area (Martin, 1965; Dott and Attig, 2004), with a predominant range of roughly 6 to $9 \mathrm{in} / \mathrm{yr}$. This is consistent with the relatively uniform underlying geology in the Driftless Area. There are somewhat low values in the central portion west of the Wisconsin
River, where the aquifers are thin and surficial soils have low permeability. These factors are expected to result in less aquifer storage and increased surface runoff. This portion of the State also has some of the lowest base-flow-index values (fig. 1) indicating that a smaller proportion of available water infiltrates and becomes recharge. Low values also are evident along the southern border of Lake Superior and the western edge of Lake Michigan, likely the result of clayey surficial soils that inhibit infiltration.

\section{Error Analysis}

The accuracy of the average annual base-flow values determined by the various techniques was evaluated using methods appropriate to each type of estimate.

To evaluate the accuracy of estimates at streamflow-gaging stations, base flow determined by the BFI approach was compared to base flow determined using manual hydrographseparation techniques. In the 1970's, hydrograph separation was performed at six streamflow-gaging stations using graphical techniques (Meyboom, 1961). For the 1967 water year, the average base flow determined by the graphical technique for these six streamflow-gaging stations was compared to average base flow determined by the BFI approach. The comparison for the individual stations is shown in table 1 .

To evaluate the accuracy of estimates for the partialrecord sites, data for a subset of streamflow-gaging stations was subjected to the appropriate analysis for the relation-line and statewide-regression approaches. From the data set of 123 streamflow-gaging stations, 40 stations were used in the regression analysis to develop the statewide-regression equation, equation 1 . Because the remaining 83 stations have estimates of average annual base flow using the BFI approach, which is assumed to be the most accurate estimate, a subset of streamflow-gaging stations was selected to evaluate the 2 approaches used at partial-record sites.

For the relation-line approach, 12 daily-discharge values during base-flow periods were selected from the continuous record at each candidate station to represent low-flow discharge measurements. A representative index station was chosen, and average annual base flow was estimated using the procedure described earlier. Based on a comparison between the annual base flow estimated using the relation-line approach and the value from the BFI approach, the average error was -2.7 percent, with a standard error of 12.6 percent. The results are shown in figure 5 .

For the statewide-regression approach, one dailydischarge value during a base-flow period was selected from the continuous record at each candidate station to represent a low-flow discharge measurement. A representative index station was chosen, and average annual base flow was estimated using the procedure described earlier. Comparing the annual 
base flow estimated using the statewide-regression approach and the value from the BFI approach, the average error was -2.4 percent, with a standard error of 14.0 percent. The results are shown in figure 6 .

Calibrated groundwater models have been developed by the USGS in a variety of study areas in Wisconsin. The recharge estimates from these models were compared to estimates produced using the methods presented in this study. Estimates of recharge for these groundwater study areas were determined using area-weighted values from streamflowgaging-station estimates (fig. 2) and partial-record estimates (fig. 4), as appropriate. The results are summarized in table 2.

Table 1. Comparison of base flow estimates from graphical and Base Flow Index methods (Institute of Hydrology, 1980a, 1980b) for six streamflow-gaging stations in Wisconsin for water year 1967.

[USGS, U.S. Geological Survey; ft³/s, cubic feet per second; R, River; nr, near]

\begin{tabular}{clcccc}
\hline \multirow{2}{*}{$\begin{array}{c}\text { USGS station } \\
\text { identification } \\
\text { number }\end{array}$} & \multicolumn{1}{c}{ Station name } & $\begin{array}{c}\text { Base flow } \\
\text { (percent of } \\
\text { streamflow) }\end{array}$ & $\begin{array}{c}\text { Graphical method } \\
\left(\mathbf{f t}^{3} / \mathbf{s}\right)\end{array}$ & $\begin{array}{c}\text { Base Flow Index method } \\
\left(\mathbf{f t}^{3} / \mathbf{s}\right)\end{array}$ & $\begin{array}{c}\text { Percent } \\
\text { difference }\end{array}$ \\
\hline 04066500 & Pike River near Amberg & 86 & 171 & 157 & -8.2 \\
04086000 & Sheboygan River at Sheboygan & 45 & 68 & 78 & +15 \\
05368000 & Hay River near Wheeler & 57 & 194 & 187 & -3.6 \\
05408000 & Kickapoo River at LaFarge & 63 & 100 & 97 & -3.0 \\
05397500 & Eau Claire River near Kelly & 49 & 126 & 81 & -36 \\
05436500 & Sugar River near Brodhead & 67 & 210 & 193 & -8.1 \\
\hline
\end{tabular}

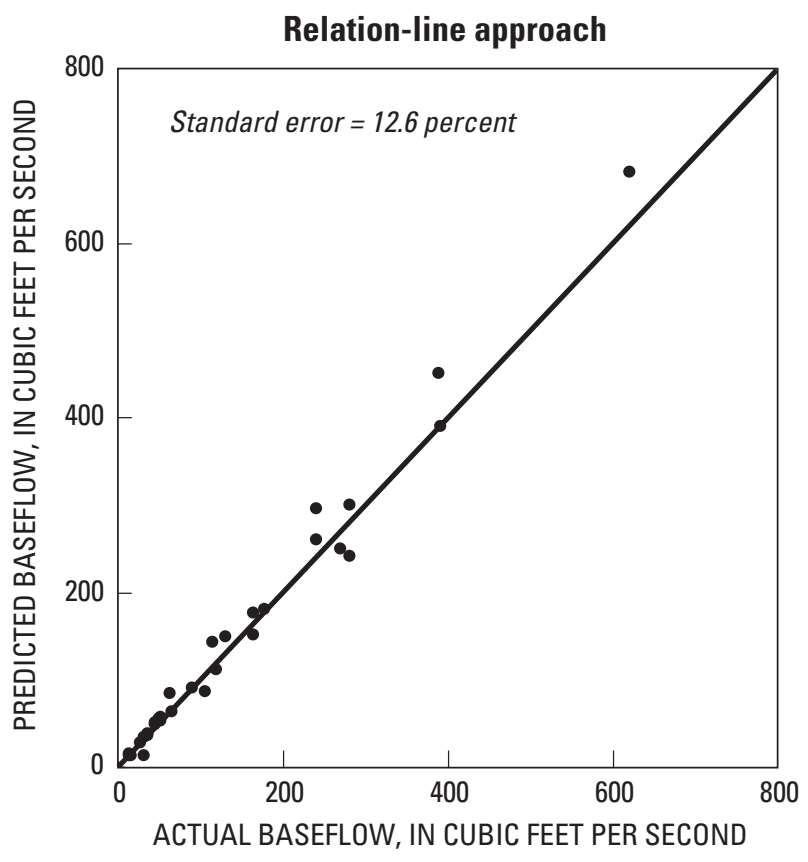

Figure 5. Comparison of annual base flow estimated using the relation-line approach (estimated) and the Base Flow Index approach (actual) at selected streamflow-gaging stations.

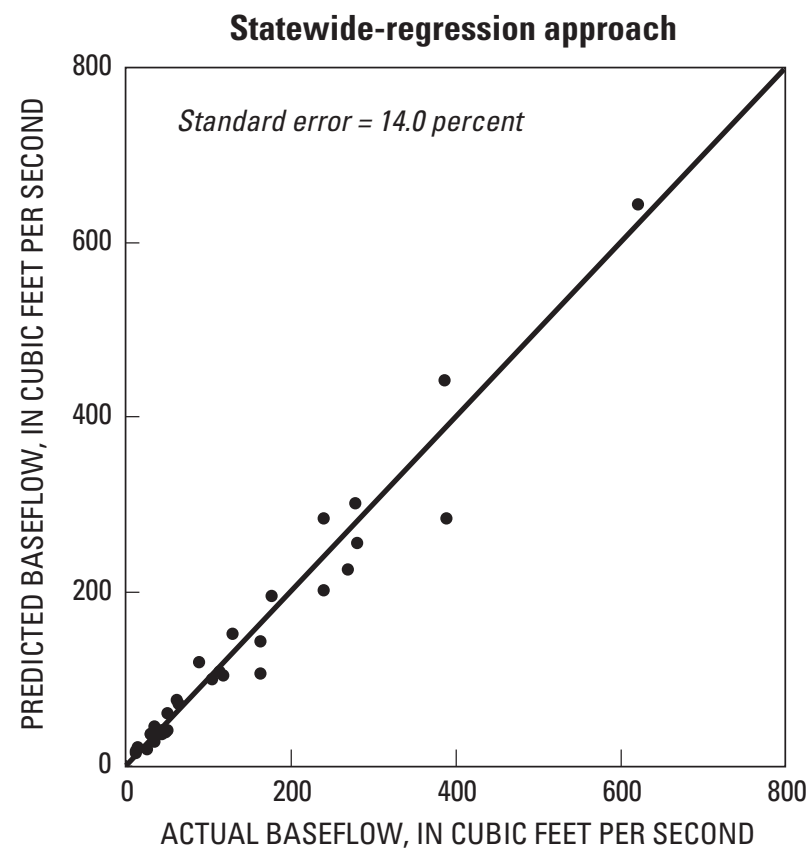

Figure 6. Comparison of annual base flow estimated using the statewide-regression approach (estimated) and the Base Flow Index approach (actual) at selected streamflow-gaging stations. 
Table 2. Comparison of recharge estimates from calibrated ground-water flow models and techniques described in this report.

[in/yr, inches per year; NA, not available]

\begin{tabular}{lcccc}
\hline \multicolumn{1}{c}{ Study area } & \multicolumn{3}{c}{$\begin{array}{c}\text { Annual recharge } \\
\text { (in/yr) }\end{array}$} & \multicolumn{1}{c}{ Reference } \\
\cline { 2 - 4 } & $\begin{array}{c}\text { Ground-water } \\
\text { model }\end{array}$ & $\begin{array}{c}\text { Streamflow-gaging } \\
\text { stations, shown in } \\
\text { figure 2 }\end{array}$ & $\begin{array}{c}\text { Partial-record } \\
\text { stations, shown in } \\
\text { figure 4 }\end{array}$ & \\
\hline Northwest Sawyer County & 10.1 & 9.2 & 18.8 & Juckem and Hunt, 2007 \\
\hline Vilas/Trout Lake & 10.5 & 10.8 & 8.8 & Hunt and others, 1998 \\
\hline Eagle River Area & 9.7 & NA & 7.2 & Graczyk and others, 2003 \\
\hline Necedah National Wildlife Refuge & 9.5 & 8.5 & NA & Hunt and others, 2000a \\
\hline Crandon Area & $17-10.2$ & 7.4 & 11.6 & Kelson and others, 2002 \\
\hline Middle Genesee Lake & 6.7 & 6.0 & 5.4 & Hunt and others, 2000b \\
\hline Pretty Lake & 5.9 & 6.6 & NA & Hunt and Krohelski, 1996 \\
\hline Dane County & 8.0 & 6.5 & Hunt and Steuer, 2000; \\
\hline Bayfield County & $22-17$ & 9.4 & 9.5 & Henz and others, 2003 \\
\hline LaCrosse County & 8.5 & 8.3 & 8.4 & Hunt and others, 2003 \\
\hline Western Vilas County & 10 & 10.1 & 9.2 & Krohelski and others, 2002 \\
\hline Washington County & $34-12$ & 5.4 & 5.1 & Dunning, 2003 \\
\hline Milwaukee County & $0.6-3$ & 4.7 & 3.2 & Dunning and others, 2004 \\
\hline Sauk County & 5.2 & 6.2 & 7.7 & Gotkowitz and others, 2002 \\
\hline Fredrick Springs & 8.0 & NA & 7.3 & Hunt and Steuer, 2000 \\
\hline
\end{tabular}

1 "Low" and "high" recharge, respectively.

2 Lake clay areas and sandy uplands, respectively.

${ }^{3}$ General and local sandy sediments, respectively.

\section{Example Applications}

The recharge values and techniques presented in this report can be used to estimate recharge at a wide variety of scales, ranging from large basin projects with areas greater than $1,000 \mathrm{mi}^{2}$ to small projects with areas less than $20 \mathrm{mi}^{2}$.

For large-scale projects, the best estimate of recharge is obtained using values for the streamflow-gaging stations. For example, to determine recharge for an area that includes most of the Trempealeau River, a general estimate of recharge for the basin is 8 to 9 in. (fig. 2). A more detailed estimate can be obtained from Appendix 6-C. (Trempealeau-Black River Basin); the recharge for Trempealeau River at Dodge (station number 05379500 ) is $8.5 \mathrm{in}$. for the $643 \mathrm{mi}^{2}$ drainage basin.

Another example for a large area would be to determine the recharge for Lafayette County. Based on inspection of Appendix 11- $A$, most of the county is within the drainage areas for four streamflow-gaging stations. The recharge values are 5 to $8 \mathrm{in}$. based on the colors of the shaded areas (Appendix
11-A). A more detailed estimate can be obtained from Appendix 11-C ( Pecatonica-Sugar River Basin). The four streamflowgaging stations that have drainage areas in Lafayette County are Galena River near Buncombe (05415000), Pecatonica River at Darlington (05432500), East Branch Pecatonica River near Blanchardville (05433000), and Pecatonica River at Martintown (05434500). The recharge values and drainage areas are $5.6 \mathrm{in}$. and $125 \mathrm{mi}^{2}, 7.1 \mathrm{in}$. and $273 \mathrm{mi}^{2}, 8.0 \mathrm{in}$. and $221 \mathrm{mi}^{2}$, and $7.9 \mathrm{in}$. and $1,034 \mathrm{mi}^{2}$, respectively. Using a weighted average based on the portion of each drainage area contained within the county, the average for Lafayette County was determined to be $6.7 \mathrm{in}$.

For smaller-scale studies, the best estimate of recharge is obtained using values for the partial-record sites. For example, to determine recharge for the City of Tomah, inspection of Appendix $7-B$ indicates there are several partial-record sites in the Tomah area. Based on Appendix 7-D (Central Wisconsin River Basin), three partial-record sites fall within a $100 \mathrm{mi}^{2}$ area centered around Tomah. The recharge values for the three 
stations (Appendix 7-D, map numbers 113, 114 and 116) are 5.2, 8.1 and 8.4 in., respectively. Using a weighted average based on the portion of each drainage area contained within the $100 \mathrm{mi}^{2}$ area $\left(30.4,6.99\right.$ and $12.9 \mathrm{mi}^{2}$, respectively), the average recharge for the Tomah area was determined to be $6.1 \mathrm{in}$.

To determine recharge for a location that is not covered by streamflow-gaging stations or partial-record sites, a streamflow measurement can be used along with the statewide equation, equation 1 , to provide an estimate. The steps required to estimate recharge are as follows:

1. Locate a discharge measurement near the location of interest by querying the miscellaneous-measurement database (http://wi.water.usgs.gov/projects/recharge/). If an appropriate measurement cannot be found, make a discharge measurement using appropriate procedures.

2. Verify that the measurement was obtained during baseflow conditions by inspecting daily flows for streamflowgaging stations near the measurement location using real-time data (http://waterdata.usgs.gov/wi/nwis/rt/) if the measurement was made within the past 18 months or historical data (http://waterdata.usgs.gov/wi/nwis/dv/) otherwise. Generally a stream will return to base-flow conditions 5 to 7 days after an appreciable increase in discharge due to a runoff event.

3. Select an appropriate nearby streamflow-gaging station to use as an index site. Determine the daily discharge on the day the measurement was made $\left(Q_{r}\right)$ and the 90 -percent flow-duration value $\left(Q_{90}\right)$ for the gage (refer to the appropriate Appendix table for the streamflow-gaging stations in the basin containing the location of interest).

4. Determine the base-flow factor from equation 2 using the discharge measurement $\left(Q_{m}\right)$ and the values determined in step 3 for the index station.

5. Determine base flow in cubic feet per second using equation 1. The estimate of recharge, in inches, is obtained using the following equation:

$$
R=\frac{13.584 \cdot Q_{b}}{A}
$$

where $R$ is recharge in inches, $Q_{b}$ is base flow in cubic feet per second, and $A$ is area in square miles.

For example to determine the recharge for the West Fork Kickapoo River at Avalanche, a query of the miscellaneous-measurement database (http://wi.water.usgs.gov/projects/recharge/) showed that no discharge measurements were available at that location; however, measurements had been made during base-flow conditions upstream and downstream of Avalanche.
For the downstream site, West Fork Kickapoo River near Westby (05408660), a discharge of $18 \mathrm{ft}^{3} / \mathrm{s}\left(Q_{m}\right)$ was measured on October 13, 1964. The drainage area $(A)$ for this site is $56.8 \mathrm{mi}^{2}$. A nearby streamflow-gaging station, Kickapoo River at La Farge (05408000), was selected as the index site. The recorded discharge at the index site on October 13, 1964 was $96 \mathrm{ft}^{3} / \mathrm{s}\left(Q_{r}^{*}\right)$, and the 90 -percent flow-duration value was $110 \mathrm{ft}^{3} / \mathrm{s}\left(Q_{90}^{*}\right)$ from Appendix 10-C). The base flow factor for the site was calculated as 0.3631 using equation (2), and the average annual base flow was calculated as $33.0 \mathrm{ft}^{3} / \mathrm{s}$ using equation (1). Using equation 3 the average annual recharge is determined to be 7.9 in.

For the upstream site, West Fork Kickapoo River near Westby (05408538), a discharge of $8.7 \mathrm{ft}^{3} / \mathrm{s}\left(Q_{m}\right)$ was measured on October 13, 1964. The drainage area $(A)$ for this site is 29.2 square miles. Using the same index station, the base flow factor for the site was calculated as 0.3414 using equation (2), and the average annual base flow was calculated as 16.2 $\mathrm{ft}^{3} / \mathrm{s}$ using equation (1). Using equation 3 the average annual recharge is determined to be 7.5 in.

\section{Selected References}

Brenden, T.O., Clark, R.D., Jr., Cooper, A.R., Seelbach, P.W., Wang, L., Aichele, S.S., Bissell, E.G., and Stewart, J.S., 2006, A GIS framework for collecting, managing, and analyzing multiscale landscape variables across large regions for river conservation and management in American Fisheries Society Symposium: American Fisheries Society, p. 49-74.

Cherkauer, D.S., 2004, Quantifying ground water recharge at multiple scales using PRMS and GIS: Ground Water, v. 42, no. 1 , p. $97-110$.

Dott, R.H., Jr., and Attig, J.W., 2004, Roadside geology of Wisconsin: Missoula, Mont., Mountain Press Publishing Company, $345 \mathrm{p}$.

Dunning, C.P., 2003, Simulation of the shallow aquifer in the vicinity of Silver Lake, Washington County, Wisconsin, using analytic elements: U.S. Geological Survey WaterResources Investigations Report 02-4204, 29 p.

Dunning, C.P., Feinstein, D.T., Hunt, R.J., and Krohelski, J.T., 2004, Simulation of ground-water flow, surface-water flow, and a deep sewer tunnel system in the Menomonee Valley, Milwaukee, Wisconsin: U.S. Geological Survey Scientific Investigations Report 2004-5031, 40 p.

Gebert, W.A., 1978, Low-flow characteristics of streams in the lower Wisconsin River basin: U.S. Geological Survey Water-Resources Investigations Report 77-118, 80 p. 
Gebert, W.A., 1979a, Red Cedar River basin, WisconsinLow-flow characteristics: U.S. Geological Survey WaterResources Investigations Report 79-29, 12 p.

Gebert, W.A., 1979b, Low-flow characteristics of streams in the Lake Superior basin, Wisconsin: U.S. Geological Survey Open-File Report 79-38, 74 p.

Gebert, W.A., 1980, Low-flow characteristics of streams in the upper Wisconsin River basin, Wisconsin: U.S. Geological Survey Open-File Report 80-691, 60 p.

Gebert, W.A., 1981, Low-flow characteristics of streams in the Central Wisconsin River Basin, Wisconsin: U.S. Geological Survey Water-Resources Investigations Report 81-495, $99 \mathrm{p}$.

Gebert, W.A., and Krug, W.R., 1996, Streamflow trends in Wisconsin's driftless area: Journal of the American Water Resources Association, v. 32, no. 4, p. 733-744.

Gebert, W.A., Radloff, M.J., Considine, E.J., and Kennedy, J.L., 2007, Use of streamflow data to estimate base flow/ ground-water recharge for Wisconsin: Journal of the American Water Resources Association, v. 43, no. 1, p. 220-236.

Gotkowitz, M., Zeiler, K. Dunning, C. and Thomas, J., 2002, Delineation of zones of contribution for municipal wells in Sauk County, Wisconsin: Wisconsin Geological and Natural History Survey Open-File Report 2002-05, 74 p.

Graczyk, D.G., Hunt, R.J., Greb, S.R., Buchwald, C.A., and Krohelski, J.T., 2003, Hydrology, water quality, and yields, from near-shore flows to four lakes in northern Wisconsin, 1999-2001: U.S. Geological Survey Water-Resources Investigations Report 03-4144, 64 p.

Holmstrom, B.K., 1978, Low-flow characteristics of streams in the Rock-Fox River basin, Wisconsin: U.S. Geological Survey Open-File Report 78-85, 98 p.

Holmstrom, B.K., 1979, Low-flow characteristics of streams in the Trempealeau-Black River basin, Wisconsin: U.S. Geological Survey Open-File Report 79-9, 79 p.

Holmstrom, B.K., 1980a, Low-flow characteristics of streams in the St. Croix River basin: U.S. Geological Survey OpenFile Report 80-696, 62 p.

Holmstrom, B.K., 1980b, Low-flow characteristics of streams in the Menominee-Oconto-Peshtigo River basin, Wisconsin: U.S. Geological Survey Open-File Report 80-749, 82 p.

Holmstrom, B.K., 1982, Low-flow characteristics of streams in the Lake Michigan basin, Wisconsin: U.S. Geological Survey Open-File Report 81-1193, 102 p.
Hunt, R.J., Anderson, M.P., and Kelson, V.A., 1998, Improving a complex finite difference groundwater-flow model through the use of an analytic element screening model: Ground Water, v. 36, no. 6, p. 1011-1017.

Hunt, R.J., Graczyk, D.J., and Rose, W.J., 2000a, Water flows in the Necedah National Wildlife Refuge, U.S. Geological Survey Fact Sheet FS-068-00, 4 p.

Hunt, R.J. and Krohelski, J.T., 1996, The application of an analytic element model to investigate groundwater-lake interactions at Pretty Lake, Wisconsin: Lakes and Reservoir Management, v. 12, no. 4, p. 487-495.

Hunt, R.J., Lin, Y., Krohelski, J.T., and Juckem, P.F., 2000b, Simulation of the shallow hydrologic system in the vicinity of Middle Genesee Lake, Wisconsin, using analytic elements and parameter estimation: U.S. Geological Survey Water-Resources Investigations Report 00-4136, 16 p.

Hunt, R.J., Saad, D.A., and Chapel, D.M., 2003, Numerical simulation of ground-water flow in La Crosse County, Wisconsin, and into nearby pools of the Mississippi River: U.S. Geological Survey Water-Resources Investigations Report 03-4154, 36 p.

Hunt, R.J., and Steuer, J.J., 2000, Simulation of the recharge area for Frederick Springs, Dane County, Wisconsin: U.S. Geological Survey Water-Resources Investigations Report 00-4172, $33 \mathrm{p}$.

Hunt, R.J., Steuer, J.J., Mansor, M.T.C., and Bullen, T.D., 2001, Delineating a recharge area for a spring using numerical modeling, Monte Carlo techniques, and geochemical investigation: Ground Water, v. 39, no 5, p. 702-712.

Institute of Hydrology, 1980a, Low flow studies: Wallingford, Oxon, United Kingdom, Report No. 1, 41 p.

Institute of Hydrology, 1980b, Low flow studies: Wallingford, Oxon, United Kingdom, Report No. 3, p. 12-19.

Juckem, P.F. and Hunt, R.J., 2007, Simulation of the shallow ground-water-flow system near Grindstone Creek and the Community of New Post, Sawyer County, Wisconsin: U.S. Geological Survey Scientific Investigations Report 2007-5014, 29 p.

Kelson, V.A., Hunt, R.J., and Haitjema, H.M., 2002, Improving a regional model using reduced complexity and parameter estimation: Ground Water, v. 40, no. 2, p. 138-149.

Krohelski, J.T., Rose, W.J., and Hunt, R.J., 2002, Hydrologic Investigation of Powell Marsh and its Relation to Dead Pike Lake, Vilas County, Wisconsin: U.S. Geological Survey Water-Resources Investigations Report 02-4034, 20 p. 
Lenz, B.N., Saad, D.A., and Fitzpatrick, F.A., 2003, Simulation of ground-water flow and rainfall runoff with emphasis on the effects of land cover, Whittlesey Creek, Bayfield County, Wisconsin, 1999-2001: U.S. Geological Survey Water-Resources Investigations Report 03-4130, 47 p.

Martin, L., 1965, The physical geography of Wisconsin: Madison, Wisc., University of Wisconsin Press, 608 p.

Meyboom, P., 1961, Estimating ground-water recharge from stream hydrographs: Groundwater Research, v. 66, p. $1203-1214$.

Rutledge, A.T., 2007, Update on the use of the RORA Program for recharge estimation: Ground Water, v. 45 , no. 3 , p. 374-382.
Scanlon, B.R., Healy, R.W., and Cook, P.G., 2002, Choosing appropriate techniques for quantifying groundwater recharge: Hydrogeology, v. 10, p. 18-39.

Stedfast, D.A., 1979, Low-flow characteristics of streams in the Pecatonica-Sugar River basin: U.S. Geological Survey Open-File Report 79-1274, 92 p.

Wahl, K.L., and Wahl, T.L., 1995, Determining the flow of Comal Springs at Braunfels, Texas, in Texas Water '95: San Antonio, Tex., August 16-17, 1995, American Society of Civil Engineers, p. 77-86. 



\section{Appendixes 1-12}

A, Locations of streamflow-gaging stations and spatial distribution of average annual recharge; $B$, locations of miscellaneous and partial-record sites and spatial distribution of estimated average annual recharge; $C$, average annual base flow and corresponding recharge, 1970-99, for streamflow-gaging stations; and $D$, average annual base flow and corresponding recharge, 1970-99, for partial-record stations.

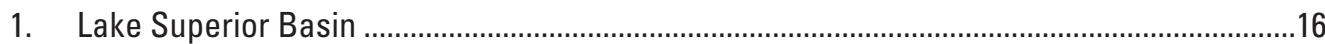

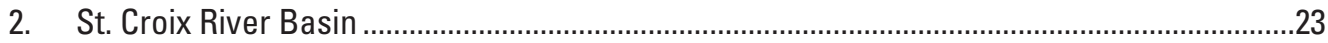

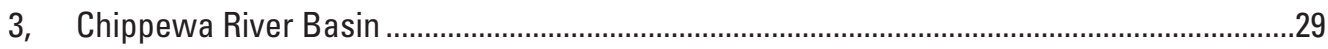

4. Upper Wisconsin River Basin ................................................................................

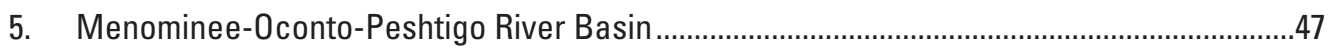

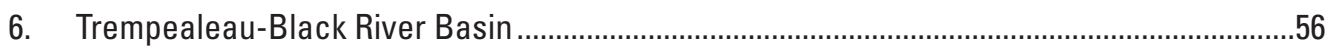

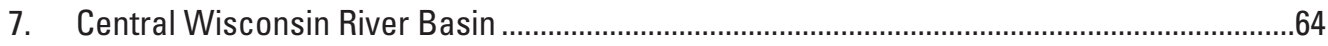

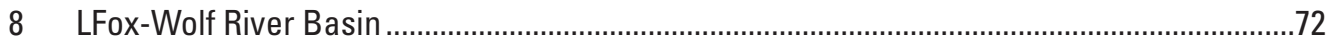

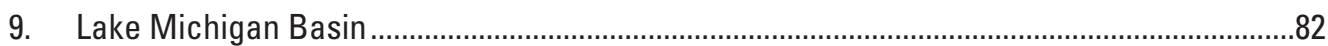

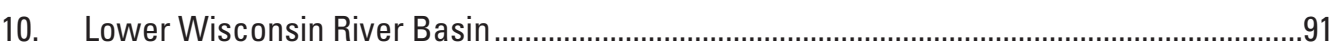

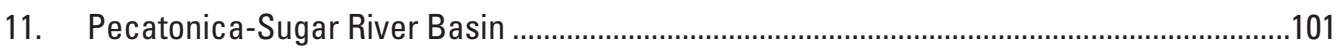

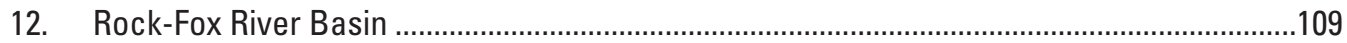




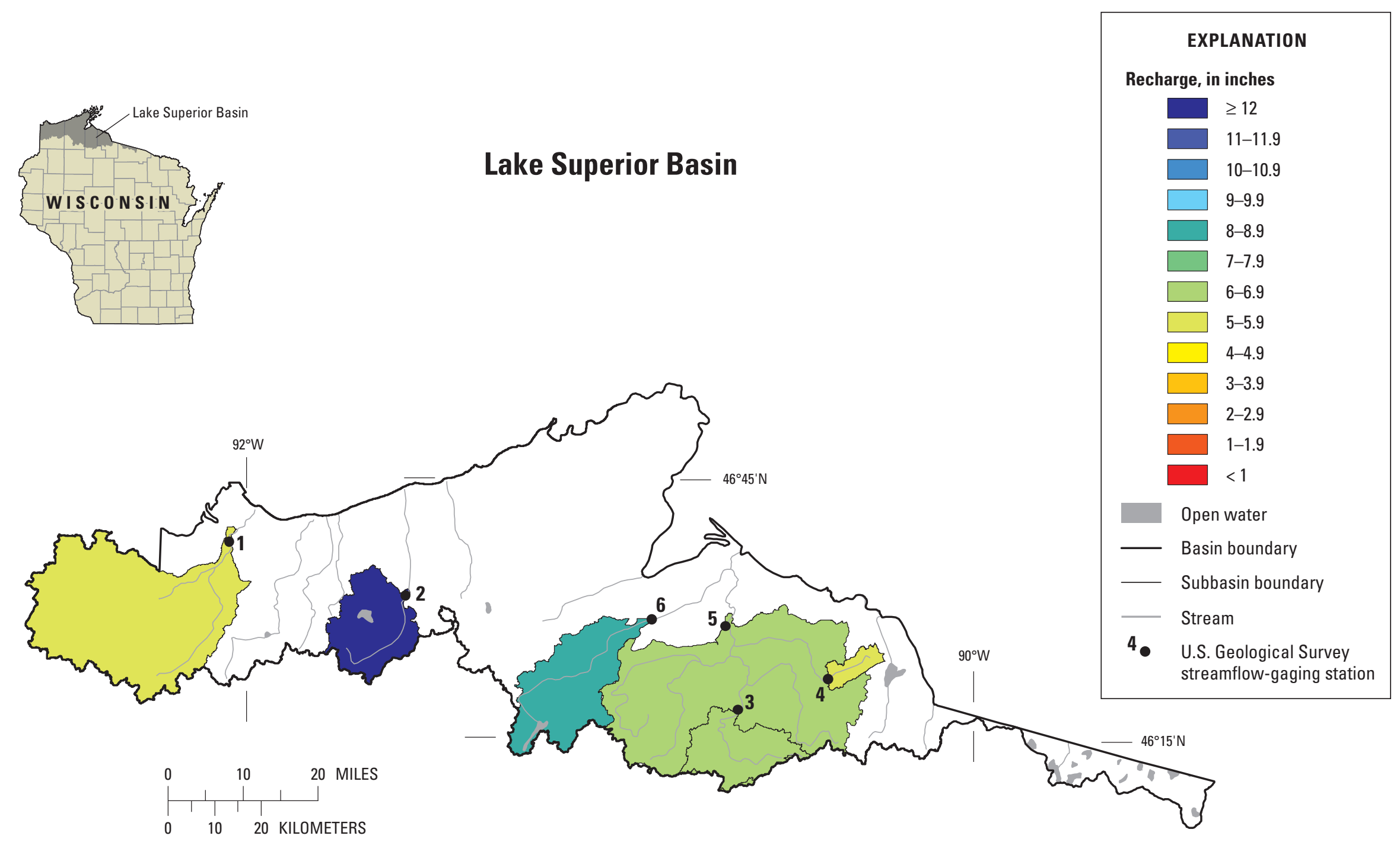

Appendix 1-A. Locations of streamflow-gaging stations in the Lake Superior Basin and spatial distribution of estimated average annual recharge. 


\section{Lake Superior Basin}

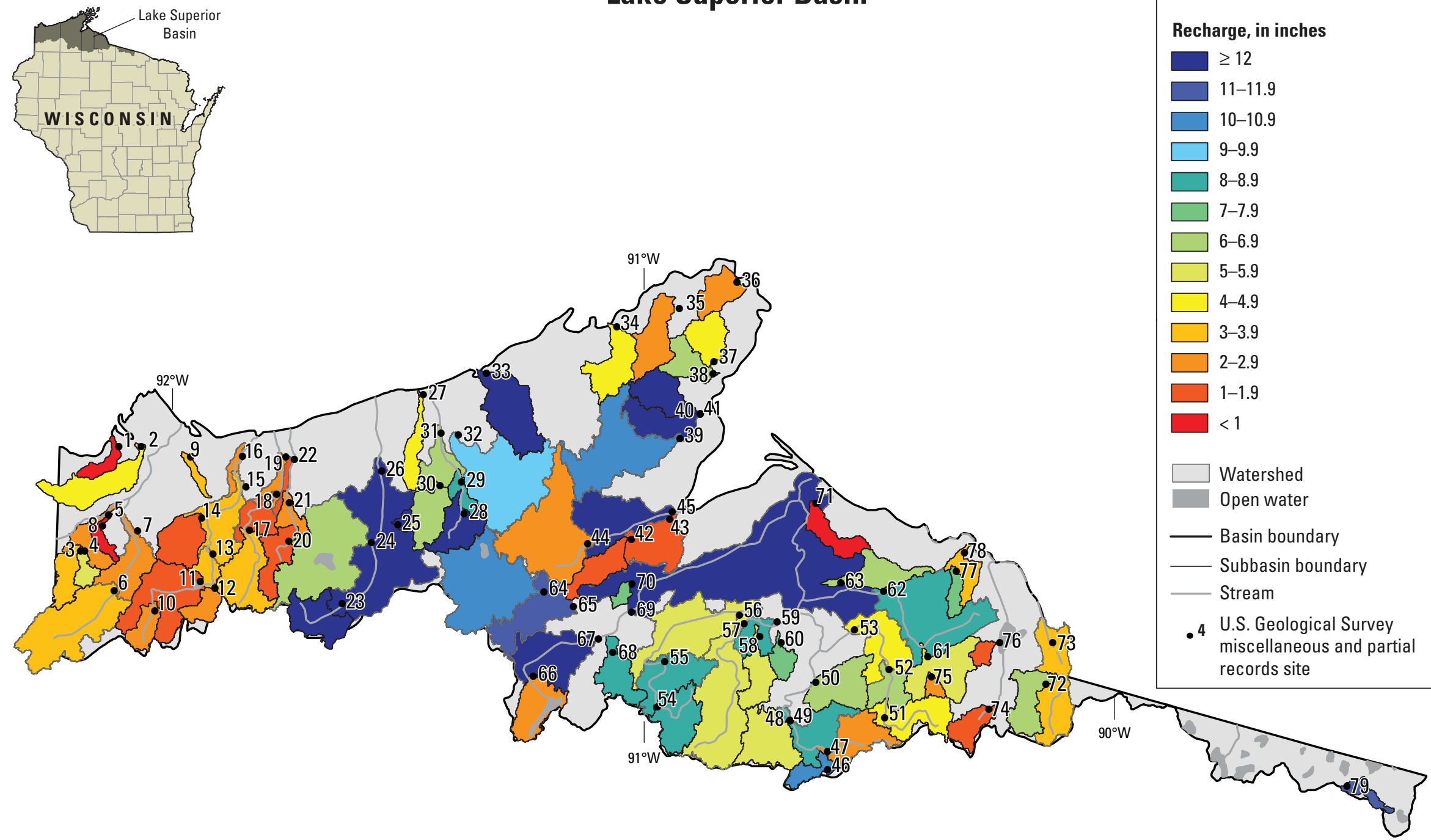


Appendix 1C. Average annual base flow and corresponding recharge, 1970-99, for streamflow-gaging stations in the Lake Superior Basin.

[USGS, U.S. Geological Survey; ID, identification number; $\mathrm{mi}^{2}$, square mile; ft³/s, cubic foot per second; BFI, Baseflow Index; ex rec, extended record; R, River; Bk, Brook; Br, Branch; Fk, Fork; Ck, Creek; Tr, Tributary; nr, near; N, North; S, South; E, East; W, West; --, not determined in historical low-flow reports. See references for full list of low-flow reports.]

\begin{tabular}{|c|c|c|c|c|c|c|c|c|c|c|c|}
\hline $\begin{array}{c}\text { Map } \\
\text { number }\end{array}$ & $\begin{array}{l}\text { USGS } \\
\text { station } \\
\text { ID }\end{array}$ & Station name & $\begin{array}{c}\text { Drainage } \\
\text { area } \\
\left(\mathrm{mi}^{2}\right)\end{array}$ & $\begin{array}{c}\text { Period of } \\
\text { analysis } \\
\text { (water years') }\end{array}$ & $\begin{array}{c}\text { Base flow } \\
\text { method }\end{array}$ & $\begin{array}{l}\text { Base flow } \\
\text { index }\end{array}$ & $\begin{array}{c}\text { Base flow } \\
\left(\mathrm{ft}^{3} / \mathrm{s}\right)\end{array}$ & $\begin{array}{c}\text { Recharge } \\
\text { (inches) }\end{array}$ & $\begin{array}{c}\text { Total } \\
\text { streamflow } \\
\left(\mathrm{ft}^{3} / \mathrm{s}\right)\end{array}$ & $\begin{array}{c}\text { Total } \\
\text { streamflow } \\
\text { (inches) }\end{array}$ & $\begin{array}{l}{ }^{2} 090 \\
\left(\mathrm{ft}^{3} / \mathrm{s}\right)\end{array}$ \\
\hline 1 & 04024430 & Nemadji R nr South Superior & 420 & 1975-1999 & BFI & 0.4060 & 164 & 5.3 & 410 & 13.1 & 63 \\
\hline 2 & 04025500 & Bois Brule $\mathrm{R}$ at Brule & 118 & 1970-1999 & BFI & .8690 & 152 & 17.5 & 180 & 20.1 & 120 \\
\hline 3 & 04026500 & Bad $\mathrm{R}$ at Mellen & 98.3 & 1949-1955 & ex rec & -- & 45 & 6.2 & -- & -- & 26 \\
\hline 4 & 04026870 & Alder Ck at Upson & 22.2 & $1973-1977$ & ex rec & -- & 9.0 & 5.5 & -- & -- & 3.0 \\
\hline 5 & 04027000 & Bad R nr Odanah & 597 & $1970-1999$ & BFI & .4850 & 301 & 6.8 & 630 & 14.3 & 130 \\
\hline 6 & 04027500 & White R nr Ashland & 301 & 1970-1999 & BFI & .6900 & 187 & 8.4 & 270 & 12.4 & 160 \\
\hline
\end{tabular}

${ }^{1}$ Water year is a 12-month period from October 1 through September 30, and is designated by the calendar year in which it ends (for example, the 2004 water year occurred October 1,2003 through September 30, 2004).

${ }^{2}$ Discharge that is exceeded 90 percent of the time. 
Appendix 1D. Average annual base flow and corresponding recharge, 1970-99, for partial-record stations in the Lake Superior Basin.

[USGS, U.S. Geological Survey; ID, identification number; $\mathrm{dd}^{\circ} \mathrm{mm}$ 'ss", degrees, minutes, seconds; $\mathrm{mi}^{2}$, square mile; $\mathrm{ft}^{3} / \mathrm{s} / \mathrm{mi}^{2}$, cubic foot per second per square mile; $\mathrm{ft}^{3} / \mathrm{s}, \mathrm{cubic} \mathrm{foot}$ per second; equation, statewide regression equation; relation, established relation line with index station; R, River; Bk, Brook; Br, Branch; Fk, Fork; Ck, Creek; Tr, Tributary; nr, near; N, North; S, South; E, East; W, West; --, not determined in historical low-flow reports. See references for full list of low-flow reports.]

\begin{tabular}{|c|c|c|c|c|c|c|c|c|c|c|}
\hline $\begin{array}{c}\text { Map } \\
\text { number }\end{array}$ & $\begin{array}{c}\text { USGS } \\
\text { station ID }\end{array}$ & Station name & $\begin{array}{c}\text { Latitude } \\
\text { (dd }{ }^{\circ} \mathrm{mm} \text { 'ss") }\end{array}$ & $\begin{array}{l}\text { Longitude } \\
\text { (ddºmm'ss") }\end{array}$ & $\begin{array}{l}\text { Index } \\
\text { station }^{1}\end{array}$ & $\begin{array}{c}\text { Drainage } \\
\text { area } \\
\left(\mathrm{mi}^{2}\right)\end{array}$ & $\begin{array}{c}\text { Base flow } \\
\text { factor } \\
\left(\mathrm{ft}^{3} / \mathrm{s} / \mathrm{mi}^{2}\right)\end{array}$ & $\begin{array}{c}\text { Base flow } \\
\text { method }\end{array}$ & $\begin{array}{c}\text { Base flow } \\
\qquad\left(\mathrm{ft}^{3} / \mathrm{s}\right)\end{array}$ & $\begin{array}{c}\text { Recharge } \\
\text { (inches) }\end{array}$ \\
\hline 1 & 04024036 & Little Pokegama R nr S Superior & $46^{\circ} 39^{\prime} 36^{\prime \prime}$ & $92^{\circ} 10^{\prime} 01^{\prime \prime}$ & 04025500 & 6.27 & 0.0032 & equation & 0.3 & 0.6 \\
\hline 2 & 04024067 & Pokegama R at S. Superior & $46^{\circ} 39^{\prime} 43^{\prime \prime}$ & $92^{\circ} 07^{\prime} 07^{\prime \prime}$ & 04025500 & 26.4 & .1071 & equation & 8.0 & 4.1 \\
\hline 3 & 04024310 & Balsam Ck nr Patzau & $46^{\circ} 30^{\prime} 13^{\prime \prime}$ & $92^{\circ} 14^{\prime} 37^{\prime \prime}$ & 05333500 & 12 & .1005 & equation & 3.5 & 3.9 \\
\hline 4 & 04024315 & Little Balsam Ck nr Patzau & $46^{\circ} 30^{\prime} 13^{\prime \prime}$ & $92^{\circ} 14^{\prime} 05^{\prime \prime}$ & 05333500 & 5.05 & .1882 & equation & 2.0 & 5.3 \\
\hline 5 & 04024325 & Balsam Ck nr Dedham & $46^{\circ} 33^{\prime} 28^{\prime \prime}$ & $92^{\circ} 11^{\prime} 05^{\prime \prime}$ & 05333500 & 29.8 & .0848 & equation & 8.0 & 3.7 \\
\hline 6 & 04024350 & Black R nr Patzau & $46^{\circ} 26^{\prime} 45^{\prime \prime}$ & $92^{\circ} 10^{\prime} 05^{\prime \prime}$ & 05333500 & 54.5 & .0170 & relation & 13.0 & 3.2 \\
\hline 7 & 04024370 & Black R nr Patzau & $46^{\circ} 32^{\prime} 09^{\prime \prime}$ & $92^{\circ} 07^{\prime} 16^{\prime \prime}$ & 05333500 & 76.7 & .0358 & equation & 13.4 & 2.4 \\
\hline 8 & 04024380 & Miller Ck Tr at Dedham & $46^{\circ} 32^{\prime} 29^{\prime \prime}$ & $92^{\circ} 11^{\prime} 52^{\prime \prime}$ & 05333500 & 4.19 & .0055 & equation & .3 & .8 \\
\hline 9 & 04024467 & Bear Ck nr S Range & $46^{\circ} 38^{\prime} 51^{\prime \prime}$ & $92^{\circ} 00^{\prime} 40^{\prime \prime}$ & 05333500 & 3.53 & .0727 & equation & .8 & 3.2 \\
\hline 10 & 04024550 & Bear Ck nr Patzua & $46^{\circ} 25^{\prime} 02^{\prime \prime}$ & $92^{\circ} 04^{\prime} 45^{\prime \prime}$ & 04025500 & 9.65 & .0331 & equation & 1.6 & 2.2 \\
\hline 11 & 04024660 & Amnicon R nr Solon Springs & $46^{\circ} 27^{\prime} 42^{\prime \prime}$ & $91^{\circ} 59^{\prime} 01^{\prime \prime}$ & 04025500 & 49.8 & .0201 & equation & 6.4 & 1.7 \\
\hline 12 & 04024710 & Cranberry Ck nr Solon Springs & $46^{\circ} 27^{\prime} 09^{\prime \prime}$ & $91^{\circ} 58^{\prime} 05^{\prime \prime}$ & 04025500 & 9.69 & .0401 & equation & 1.7 & 2.4 \\
\hline 13 & 04024790 & Amnicon R nr Hines & $46^{\circ} 30^{\prime} 13^{\prime \prime}$ & $91^{\circ} 57^{\prime} 27^{\prime \prime}$ & 04025500 & 65.4 & .0642 & equation & 15.5 & 3.2 \\
\hline 14 & 04024810 & Little Amnicon R nr S Range & $46^{\circ} 33^{\prime} 25^{\prime \prime}$ & $91^{\circ} 59^{\prime} 00^{\prime \prime}$ & 04025500 & 19.8 & .0186 & equation & 2.4 & 1.6 \\
\hline 15 & 04025000 & Amnicon R nr Poplar & $46^{\circ} 36^{\prime} 20^{\prime \prime}$ & $91^{\circ} 53^{\prime} 20^{\prime \prime}$ & 04025500 & 110 & -- & relation & 25.0 & 3.1 \\
\hline 16 & 04025010 & Amnicon R nr S Range & $46^{\circ} 39^{\prime} 00^{\prime \prime}$ & $91^{\circ} 53^{\prime} 54^{\prime \prime}$ & 04025500 & 115 & .0531 & equation & 24.9 & 2.9 \\
\hline 17 & 04025057 & Middle R nr Hines & $46^{\circ} 32^{\prime} 27^{\prime \prime}$ & $91^{\circ} 52^{\prime} 44^{\prime \prime}$ & 04025500 & 28.2 & -- & relation & 6.6 & 3.2 \\
\hline 18 & 04025070 & Middle R nr Poplar & $46^{\circ} 35^{\prime} 42^{\prime \prime}$ & $91^{\circ} 49^{\prime} 22^{\prime \prime}$ & 04025500 & 43.1 & .0386 & equation & 7.8 & 2.4 \\
\hline 19 & 04025100 & Middle R nr Poplar & $46^{\circ} 39^{\prime} 05^{\prime \prime}$ & $91^{\circ} 48^{\prime} 15^{\prime \prime}$ & 04025500 & 48.4 & .0850 & relation & 7.8 & 2.2 \\
\hline 20 & 04025110 & Poplar R nr Hawthorne & $46^{\circ} 31^{\prime} 32^{\prime \prime}$ & $91^{\circ} 47^{\prime} 38^{\prime \prime}$ & 04025500 & 13.8 & .0137 & equation & 1.4 & 1.4 \\
\hline 21 & 04025120 & Poplar R at Poplar & $46^{\circ} 34^{\prime} 58^{\prime \prime}$ & $91^{\circ} 47^{\prime} 41^{\prime \prime}$ & 04025500 & 26.3 & .0273 & equation & 3.9 & 2.0 \\
\hline 22 & 04025140 & Poplar R nr Poplar & $46^{\circ} 38^{\prime} 54^{\prime \prime}$ & $91^{\circ} 47^{\prime} 08^{\prime \prime}$ & 04025500 & 31.1 & .0183 & equation & 3.8 & 1.6 \\
\hline 23 & 04025300 & Bois Brule R nr Solon Springs & $46^{\circ} 26^{\prime} 02^{\prime \prime}$ & $91^{\circ} 40^{\prime} 32^{\prime \prime}$ & 04025500 & 24.2 & 1.0709 & equation & 24.2 & 13.6 \\
\hline 24 & 04025450 & Nebagamon Ck nr Brule & $46^{\circ} 31^{\prime} 34^{\prime \prime}$ & $91^{\circ} 366^{\prime \prime}$ & 04025500 & 60.2 & .2186 & equation & 26.9 & 6.1 \\
\hline
\end{tabular}

${ }^{1}$ Low-flow discharge measurements at partial-record stations were compared to one or more nearby USGS gaging stations to establish a relation between measured discharge and concurrent daily discharge. The nearby gaging station that provided the best relation with the partial-record station was chosen as the index station and was associated with nearby miscellaneous measurement sites.

${ }^{2}$ Mismatch between surface-water and ground-water drainage area. 
Appendix 1D. Average annual base flow and corresponding recharge, 1970-99, for partial-record stations in the Lake Superior Basin-Continued.

[USGS, U.S. Geological Survey; ID, identification number; $\mathrm{dd}^{\circ} \mathrm{mm}$ 'ss", degrees, minutes, seconds; $\mathrm{mi}^{2}$, square mile; $\mathrm{ft}^{3} / \mathrm{s} / \mathrm{mi}^{2}$, cubic foot per second per square mile; $\mathrm{ft}^{3} / \mathrm{s}$, cubic foot per second; equation, statewide regression equation; relation, established relation line with index station; R, River; Bk, Brook; Br, Branch; Fk, Fork; Ck, Creek; Tr, Tributary; nr, near; N, North; S, South; E, East; W, West; ,-- not determined in historical low-flow reports. See references for full list of low-flow reports.]

\begin{tabular}{|c|c|c|c|c|c|c|c|c|c|c|}
\hline $\begin{array}{c}\text { Map } \\
\text { number }\end{array}$ & $\begin{array}{c}\text { USGS } \\
\text { station ID }\end{array}$ & Station name & $\begin{array}{c}\text { Latitude } \\
\text { (ddºmm'ss") }\end{array}$ & $\begin{array}{c}\text { Longitude } \\
\text { (ddºmm'ss") }\end{array}$ & $\begin{array}{l}\text { Index } \\
\text { station }^{1}\end{array}$ & $\begin{array}{c}\text { Drainage } \\
\text { area } \\
\left(\mathrm{mi}^{2}\right)\end{array}$ & $\begin{array}{c}\text { Base flow } \\
\text { factor } \\
\left(\mathrm{ft}^{3} / \mathrm{s} / \mathrm{mi}^{2}\right)\end{array}$ & $\begin{array}{l}\text { Base flow } \\
\text { method }\end{array}$ & $\begin{array}{c}\text { Base flow } \\
\quad\left(\mathrm{ft}^{3} / \mathrm{s}\right)\end{array}$ & $\begin{array}{c}\text { Recharge } \\
\text { (inches) }\end{array}$ \\
\hline 25 & 04025518 & Sandy Run at Brule & $46^{\circ} 33^{\prime} 13^{\prime \prime}$ & 91³3'33" & 04025500 & 2.53 & -- & relation & 4.9 & ${ }^{2} 26.3$ \\
\hline 26 & 04026001 & Bois Brule R nr Brule & $46^{\circ} 37^{\prime} 59^{\prime \prime}$ & $91^{\circ} 35^{\prime} 49^{\prime \prime}$ & 04025500 & 162 & 0.8614 & equation & 150.5 & 12.6 \\
\hline 27 & 04026030 & Reefer Ck nr Port Wing & $46^{\circ} 44^{\prime} 54^{\prime \prime}$ & $91^{\circ} 30^{\prime} 32^{\prime \prime}$ & 04025500 & 11.6 & .2900 & relation & 3.7 & 4.3 \\
\hline 28 & 04026040 & Iron $\mathrm{R}$ at Iron $\mathrm{R}$ & $46^{\circ} 34^{\prime} 16^{\prime \prime}$ & $91^{\circ} 24^{\prime} 59^{\prime \prime}$ & 04025500 & 15 & -- & relation & 14.0 & ${ }^{2} 12.7$ \\
\hline 29 & 04026045 & Iron $\mathrm{R} n \mathrm{nr}$ Iron $\mathrm{R}$ & $46^{\circ} 37^{\prime} 08^{\prime \prime}$ & $91^{\circ} 25^{\prime} 24^{\prime \prime}$ & 04025500 & 24.4 & .4690 & equation & 15.9 & 8.9 \\
\hline 30 & 04026060 & Muskeg Ck nr Iron R & $46^{\circ} 36^{\prime} 47^{\prime \prime}$ & $91^{\circ} 28^{\prime} 11^{\prime \prime}$ & 04025500 & 17.9 & .2974 & equation & 9.2 & 6.9 \\
\hline 31 & 04026070 & Iron $\mathrm{R}$ nriron $\mathrm{R}$ & $46^{\circ} 41^{\prime} 31^{\prime \prime}$ & $91^{\circ} 28^{\prime} 07^{\prime \prime}$ & 04025500 & 58.5 & .3458 & equation & 33.1 & 7.7 \\
\hline 32 & 04026110 & E Fk nr Iron R & $46^{\circ} 41^{\prime} 23^{\prime \prime}$ & $91^{\circ} 25^{\prime} 54^{\prime \prime}$ & 04025500 & 56.7 & .4937 & equation & 38.6 & 9.3 \\
\hline 33 & 04026120 & Flag R, Flag R at Port Wing & $46^{\circ} 46^{\prime} 55^{\prime \prime}$ & $91^{\circ} 22^{\prime} 25^{\prime \prime}$ & 04025500 & 31.75 & .9260 & relation & 32.0 & ${ }^{2} 13.7$ \\
\hline 34 & 04026160 & Siskiwit R at Cornucopia & $46^{\circ} 51^{\prime} 17^{\prime \prime}$ & $91^{\circ} 05^{\prime} 29^{\prime \prime}$ & 04025500 & 47.4 & .1386 & equation & 16.6 & 4.8 \\
\hline 35 & 04026190 & Sand R nr Red Cliff & $46^{\circ} 53^{\prime} 00^{\prime \prime}$ & $90^{\circ} 57^{\prime} 20^{\prime \prime}$ & 04025500 & 27.4 & .1550 & relation & 5.8 & 2.9 \\
\hline 36 & 04026215 & Raspberry R nr Sand Bay & $46^{\circ} 55^{\prime} 24^{\prime \prime}$ & $90^{\circ} 49^{\prime} 51^{\prime \prime}$ & 04025500 & 13.8 & .0462 & equation & 2.7 & 2.6 \\
\hline 37 & 04026230 & N Pikes Ck nr Bayfield & $46^{\circ} 48^{\prime} 17^{\prime \prime}$ & $90^{\circ} 52^{\prime} 45^{\prime \prime}$ & 04027000 & 15.1 & .1134 & equation & 4.7 & 4.2 \\
\hline 38 & 040262301 & Pikes Ck nr Bayfield & $46^{\circ} 47^{\prime} 08^{\prime \prime}$ & $90^{\circ} 52^{\prime} 53^{\prime \prime}$ & 04027000 & 30.2 & -- & relation & 15.0 & 6.7 \\
\hline 39 & 04026300 & Sioux R nr Washburn & $46^{\circ} 41^{\prime} 20^{\prime \prime}$ & $90^{\circ} 57^{\prime} 02^{\prime \prime}$ & 04027000 & 13.6 & .2400 & relation & 10.5 & 10.5 \\
\hline 40 & 04026309 & Sioux R nr Washburn & $46^{\circ} 43^{\prime} 32^{\prime \prime}$ & $90^{\circ} 54^{\prime} 27^{\prime \prime}$ & 04027000 & 51.3 & 1.0240 & equation & 51.0 & ${ }^{2} 13.5$ \\
\hline 41 & 04026315 & Little Sioux R nr Washburn & $46^{\circ} 43^{\prime} 36^{\prime \prime}$ & $90^{\circ} 54^{\prime} 26^{\prime \prime}$ & 04027000 & 19.1 & 1.4110 & equation & 22.0 & ${ }^{2} 15.6$ \\
\hline 42 & 04026335 & S Fish Ck nr Benoit & $46^{\circ} 32^{\prime} 15^{\prime \prime}$ & $91^{\circ} 03^{\prime} 11^{\prime \prime}$ & 04025500 & 17.1 & .0078 & equation & 1.3 & 1.0 \\
\hline 43 & 04026341 & S Fish Ck nr Ashland & $46^{\circ} 34^{\prime} 06^{\prime \prime}$ & $90^{\circ} 58^{\prime} 14^{\prime \prime}$ & 04025500 & 38.4 & .0112 & equation & 3.6 & 1.3 \\
\hline 44 & 04026346 & N Fish Ck nr Benoit & $46^{\circ} 31^{\prime} 49^{\prime \prime}$ & $91^{\circ} 08^{\prime} 51^{\prime \prime}$ & 04025500 & 36 & .0389 & equation & 6.5 & 2.4 \\
\hline 45 & 04026350 & N Fish Ck nr Ashland & $46^{\circ} 34^{\prime} 45^{\prime \prime}$ & $90^{\circ} 57^{\prime} 55^{\prime \prime}$ & 04025500 & 74.4 & .9290 & relation & 80.0 & ${ }^{2} 14.6$ \\
\hline 46 & 04026400 & Spillerberg Ck nr Cayuga & $46^{\circ} 11^{\prime} 48^{\prime \prime}$ & $90^{\circ} 37^{\prime} 32^{\prime \prime}$ & 04027000 & 6.59 & .2270 & relation & 5.2 & 10.7 \\
\hline 47 & 04026410 & Bad $\mathrm{R}$ at Morse & $46^{\circ} 13^{\prime} 27^{\prime \prime}$ & $90^{\circ} 37^{\prime} 32^{\prime \prime}$ & 04027000 & 32.1 & .1167 & equation & 10.2 & 4.3 \\
\hline 48 & 04026449 & Iron $\mathrm{R}$ nr Mellen & $46^{\circ} 16^{\prime} 11^{\prime \prime}$ & $90^{\circ} 42^{\prime} 23^{\prime \prime}$ & 04027000 & 26.7 & .1916 & equation & 11.0 & 5.6 \\
\hline
\end{tabular}

${ }^{1}$ Low-flow discharge measurements at partial-record stations were compared to one or more nearby USGS gaging stations to establish a relation between measured discharge and concurrent daily discharge. The nearby gaging station that provided the best relation with the partial-record station was chosen as the index station and was associated with nearby miscellaneous measurement sites

${ }^{2}$ Mismatch between surface-water and ground-water drainage area. 
Appendix 1D. Average annual base flow and corresponding recharge, 1970-99, for partial-record stations in the Lake Superior Basin-Continued.

[USGS, U.S. Geological Survey; ID, identification number; $\mathrm{dd}^{\circ} \mathrm{mm}$ 'ss", degrees, minutes, seconds; $\mathrm{mi}^{2}$, square mile; $\mathrm{ft}^{3} / \mathrm{s} / \mathrm{mi}^{2}$, cubic foot per second per square mile; $\mathrm{ft}^{3} / \mathrm{s}$, cubic foot per second; equation, statewide regression equation; relation, established relation line with index station; R, River; Bk, Brook; Br, Branch; Fk, Fork; Ck, Creek; Tr, Tributary; nr, near; N, North; S, South; E, East; W, West; --, not determined in historical low-flow reports. See references for full list of low-flow reports.]

\begin{tabular}{|c|c|c|c|c|c|c|c|c|c|c|}
\hline $\begin{array}{c}\text { Map } \\
\text { number }\end{array}$ & $\begin{array}{c}\text { USGS } \\
\text { station ID }\end{array}$ & Station name & $\begin{array}{c}\text { Latitude } \\
\text { (dd }{ }^{\circ} \mathrm{mm} \text { 'ss") }\end{array}$ & $\begin{array}{l}\text { Longitude } \\
\text { (dd }{ }^{\circ} \text { mm'ss") }\end{array}$ & $\begin{array}{l}\text { Index } \\
\text { station }^{1}\end{array}$ & $\begin{array}{c}\text { Drainage } \\
\text { area } \\
\left(\mathrm{mi}^{2}\right)\end{array}$ & $\begin{array}{c}\text { Base flow } \\
\text { factor } \\
\left(\mathrm{ft}^{3} / \mathrm{s} / \mathrm{mi}^{2}\right)\end{array}$ & $\begin{array}{c}\text { Base flow } \\
\text { method }\end{array}$ & $\begin{array}{c}\text { Base flow } \\
\left(\mathrm{ft}^{3} / \mathrm{s}\right)\end{array}$ & $\begin{array}{c}\text { Recharge } \\
\text { (inches) }\end{array}$ \\
\hline 49 & 04026450 & Bad R nr Mellen & $46^{\circ} 16^{\prime} 14^{\prime \prime}$ & $90^{\circ} 42^{\prime} 26^{\prime \prime}$ & 04027000 & 82 & 0.2270 & BFI & 44.0 & 7.3 \\
\hline 50 & 04026510 & Montreal Ck at Mellen & $46^{\circ} 19^{\prime} 36^{\prime \prime}$ & $90^{\circ} 39^{\prime} 08^{\prime \prime}$ & 04027000 & 26 & .2776 & equation & 12.9 & 6.8 \\
\hline 51 & 04026530 & Tyler Fks R nr Mellen & $46^{\circ} 16^{\prime} 29^{\prime \prime}$ & $90^{\circ} 30^{\prime} 11^{\prime \prime}$ & 04027000 & 21.4 & .1391 & equation & 7.4 & 4.7 \\
\hline 52 & 04026550 & Tyler Fks R nr Upson & $46^{\circ} 20^{\prime} 50^{\prime \prime}$ & $90^{\circ} 29^{\prime} 40^{\prime \prime}$ & 04027000 & 36.9 & .1400 & relation & 19.0 & 7.0 \\
\hline 53 & 04026560 & Tyler Fks R nr Mellen & $46^{\circ} 24^{\prime} 20^{\prime \prime}$ & $90^{\circ} 34^{\prime} 09^{\prime \prime}$ & 04027000 & 64.9 & .1492 & equation & 23.8 & 5.0 \\
\hline 54 & 04026580 & Marengo R nr Grandview & $46^{\circ} 17^{\prime} 20^{\prime \prime}$ & $90^{\circ} 59^{\prime} 31 "$ & 04027000 & 25.5 & .3917 & equation & 15.2 & 8.1 \\
\hline 55 & 04026590 & Marengo R nr Grandview & $46^{\circ} 21^{\prime} 20^{\prime \prime}$ & $90^{\circ} 58^{\prime} 41^{\prime \prime}$ & 04027000 & 50.5 & .3878 & equation & 30.3 & 8.1 \\
\hline 56 & 04026600 & Marengo R at Marengo & $46^{\circ} 25^{\prime} 35^{\prime \prime}$ & $90^{\circ} 49^{\prime} 05^{\prime \prime}$ & 04027000 & 101 & .6060 & relation & 52.0 & 7.0 \\
\hline 57 & 04026620 & Brunsweiler $\mathrm{R}$ at Marengo & $46^{\circ} 24^{\prime} 47^{\prime \prime}$ & $90^{\circ} 48^{\prime} 28^{\prime \prime}$ & 04027000 & 63.4 & .2118 & equation & 27.9 & 6.0 \\
\hline 58 & 04026650 & Trout R nr High Bridge & $46^{\circ} 23^{\prime} 40^{\prime \prime}$ & $90^{\circ} 46 ' 25^{\prime \prime}$ & 04027000 & 13.5 & .2110 & relation & 5.6 & 5.6 \\
\hline 59 & 04026740 & Marengo R nr Mellen & $46^{\circ} 25^{\prime} 00^{\prime \prime}$ & $90^{\circ} 44^{\prime} 13^{\prime \prime}$ & 04027000 & 195 & .4048 & equation & 122.8 & 8.6 \\
\hline 60 & 04026760 & Silver Ck nr Mellen & $46^{\circ} 23^{\prime} 12^{\prime \prime}$ & $90^{\circ} 43^{\prime} 37^{\prime \prime}$ & 04027000 & 7.69 & .3412 & equation & 4.2 & 7.3 \\
\hline 61 & 04026859 & Potato R at Upson & $46^{\circ} 21^{\prime} 59^{\prime \prime}$ & $90^{\circ} 24^{\prime} 38^{\prime \prime}$ & 04027000 & 31.1 & .1595 & equation & 11.6 & 5.1 \\
\hline 62 & 04026900 & Potato R at Gurney & $46^{\circ} 27^{\prime} 50^{\prime \prime}$ & $90^{\circ} 30^{\prime} 25^{\prime \prime}$ & 04027000 & 93.1 & .2190 & relation & 45.0 & 6.6 \\
\hline 63 & 04026950 & Vaughncreek nr Gurney & $46^{\circ} 28^{\prime} 33^{\prime \prime}$ & $90^{\circ} 36^{\prime} 00^{\prime \prime}$ & 04027000 & 18 & .2597 & equation & 8.6 & 6.5 \\
\hline 64 & 04027045 & White R nr Delta & $46^{\circ} 27^{\prime} 25^{\prime \prime}$ & $91^{\circ} 14^{\prime} 25^{\prime \prime}$ & 04025500 & 106 & .5665 & equation & 78.5 & 10.1 \\
\hline 65 & 04027095 & White R nr Mason & $46^{\circ} 26^{\prime} 10^{\prime \prime}$ & $91^{\circ} 10^{\prime} 35^{\prime \prime}$ & 04025500 & 127 & .6823 & equation & 104.0 & 11.1 \\
\hline 66 & 04027110 & Long $\mathrm{Lk} \mathrm{Br}$ at Drummond & $46^{\circ} 29^{\prime} 17^{\prime \prime}$ & $91^{\circ} 16^{\prime} 45^{\prime \prime}$ & 04025500 & 23.4 & .0551 & equation & 5.0 & 2.9 \\
\hline 67 & 04027140 & Long Lk Br nr Grandview & $46^{\circ} 23^{\prime} 19^{\prime \prime}$ & $91^{\circ} 07^{\prime} 17^{\prime \prime}$ & 04027000 & 54.5 & 1.1370 & equation & 57.2 & 14.3 \\
\hline 68 & 04027200 & Twenty Mile Ck at Grandview & $46^{\circ} 22^{\prime} 05^{\prime \prime}$ & $91^{\circ} 05^{\prime} 27^{\prime \prime}$ & 04027000 & 18.7 & .4940 & relation & 12.0 & 8.7 \\
\hline 69 & 04027250 & White $\mathrm{R}$ at Mason & $46^{\circ} 25^{\prime} 47^{\prime \prime}$ & $91^{\circ} 03^{\prime} 02^{\prime \prime}$ & 04027000 & 264 & 1.1527 & equation & 288.2 & 14.8 \\
\hline 70 & 04027265 & Spring Crek nr Mason & $46^{\circ} 28^{\prime} 16^{\prime \prime}$ & $91^{\circ} 03^{\prime} 02^{\prime \prime}$ & 04027000 & 3.35 & .3620 & equation & 1.8 & 7.4 \\
\hline 71 & 04027650 & Denomie Ck nr Odanah & $46^{\circ} 35^{\prime} 40^{\prime \prime}$ & $90^{\circ} 39^{\prime} 17^{\prime \prime}$ & 04027000 & 13.9 & .0016 & equation & .5 & .5 \\
\hline 72 & 04027900 & Laymans Ck nr Hurley & $46^{\circ} 19^{\prime} 35^{\prime \prime}$ & $90^{\circ} 09^{\prime} 20^{\prime \prime}$ & 04027000 & 13.8 & .0320 & relation & 6.4 & 6.3 \\
\hline
\end{tabular}

${ }^{1}$ Low-flow discharge measurements at partial-record stations were compared to one or more nearby USGS gaging stations to establish a relation between measured discharge and concurrent daily discharge. The nearby gaging station that provided the best relation with the partial-record station was chosen as the index station and was associated with nearby miscellaneous measurement sites.

${ }^{2}$ Mismatch between surface-water and ground-water drainage area. 
Appendix 1D. Average annual base flow and corresponding recharge, 1970-99, for partial-record stations in the Lake Superior Basin-Continued.

[USGS, U.S. Geological Survey; ID, identification number; $\mathrm{dd}^{\circ} \mathrm{mm}$ 'ss", degrees, minutes, seconds; $\mathrm{mi}^{2}$, square mile; $\mathrm{ft}^{3} / \mathrm{s} / \mathrm{mi}^{2}$, cubic foot per second per square mile; $\mathrm{ft}^{3} / \mathrm{s}$, cubic foot per second; equation, statewide regression equation; relation, established relation line with index station; R, River; Bk, Brook; Br, Branch; Fk, Fork; Ck, Creek; Tr, Tributary; nr, near; N, North; S, South; E, East; W, West; ,-- not determined in historical low-flow reports. See references for full list of low-flow reports.]

\begin{tabular}{|c|c|c|c|c|c|c|c|c|c|c|}
\hline $\begin{array}{c}\text { Map } \\
\text { number }\end{array}$ & $\begin{array}{c}\text { USGS } \\
\text { station ID }\end{array}$ & Station name & $\begin{array}{c}\text { Latitude } \\
\text { (dd }{ }^{\circ} \mathrm{mm} \text { 'ss") }\end{array}$ & $\begin{array}{c}\text { Longitude } \\
\text { (dd }{ }^{\circ} \mathrm{mm} \text { 'ss") }\end{array}$ & $\begin{array}{l}\text { Index } \\
\text { station }^{1}\end{array}$ & $\begin{array}{c}\text { Drainage } \\
\text { area } \\
\left(\mathrm{mi}^{2}\right)\end{array}$ & $\begin{array}{c}\text { Base flow } \\
\text { factor } \\
\left(\mathrm{ft}^{3} / \mathrm{s} / \mathrm{mi}^{2}\right)\end{array}$ & $\begin{array}{c}\text { Base flow } \\
\text { method }\end{array}$ & $\begin{array}{c}\text { Base flow } \\
\left(\mathrm{ft}^{3} / \mathrm{s}\right)\end{array}$ & $\begin{array}{c}\text { Recharge } \\
\text { (inches) }\end{array}$ \\
\hline 73 & 04027950 & Montreal Ck nr Hurley & $46^{\circ} 23^{\prime} 15^{\prime \prime}$ & $90^{\circ} 08^{\prime} 29^{\prime \prime}$ & 04027000 & 49.1 & 0.1060 & equation & 15.0 & 4.1 \\
\hline 74 & 04028650 & W Fk Montreal R nr Upson & $46^{\circ} 24^{\prime} 49^{\prime \prime}$ & $90^{\circ} 24^{\prime} 16^{\prime \prime}$ & 04027000 & 10.5 & .0273 & equation & 1.5 & 2.0 \\
\hline 75 & 04028710 & W Fk Montreal R Tr nr Montreal & $46^{\circ} 20^{\prime} 10^{\prime \prime}$ & $90^{\circ} 24^{\prime} 11^{\prime \prime}$ & 04027000 & 7.38 & .0598 & equation & 1.6 & 3.0 \\
\hline 76 & 04028985 & E R nr Montreal & $46^{\circ} 23^{\prime} 15^{\prime \prime}$ & $90^{\circ} 15^{\prime} 22^{\prime \prime}$ & 04027000 & 4.73 & .0093 & equation & .4 & 1.1 \\
\hline 77 & 04029700 & Boomer Ck nr Saxon & $46^{\circ} 29^{\prime} 40^{\prime \prime}$ & $90^{\circ} 21^{\prime} 02^{\prime \prime}$ & 04027000 & 5.33 & .0280 & relation & 2.8 & 7.1 \\
\hline 78 & 04029780 & Fourche Ck nr Saxon & $46^{\circ} 21^{\prime} 30^{\prime \prime}$ & $90^{\circ} 15^{\prime} 55^{\prime \prime}$ & 04027000 & 14.9 & .0799 & equation & 3.8 & 3.5 \\
\hline 79 & 04038400 & S Br Ontonogon R nr Boulder Jct & $46^{\circ} 10^{\prime} 24^{\prime \prime}$ & $89^{\circ} 45^{\prime} 32^{\prime \prime}$ & 05359500 & 6.14 & .8384 & equation & 5.3 & 11.7 \\
\hline
\end{tabular}

${ }^{1}$ Low-flow discharge measurements at partial-record stations were compared to one or more nearby USGS gaging stations to establish a relation between measured discharge and concurrent daily discharge. The nearby gaging station that provided the best relation with the partial-record station was chosen as the index station and was associated with nearby miscellaneous measurement sites.

${ }^{2}$ Mismatch between surface-water and ground-water drainage area. 


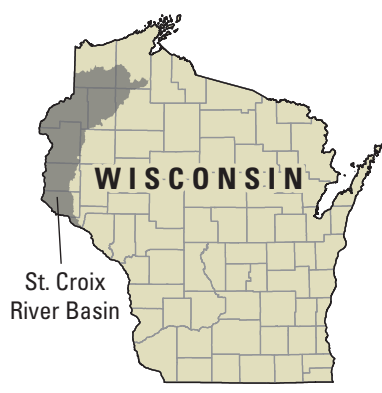

\section{St. Croix River Basin}

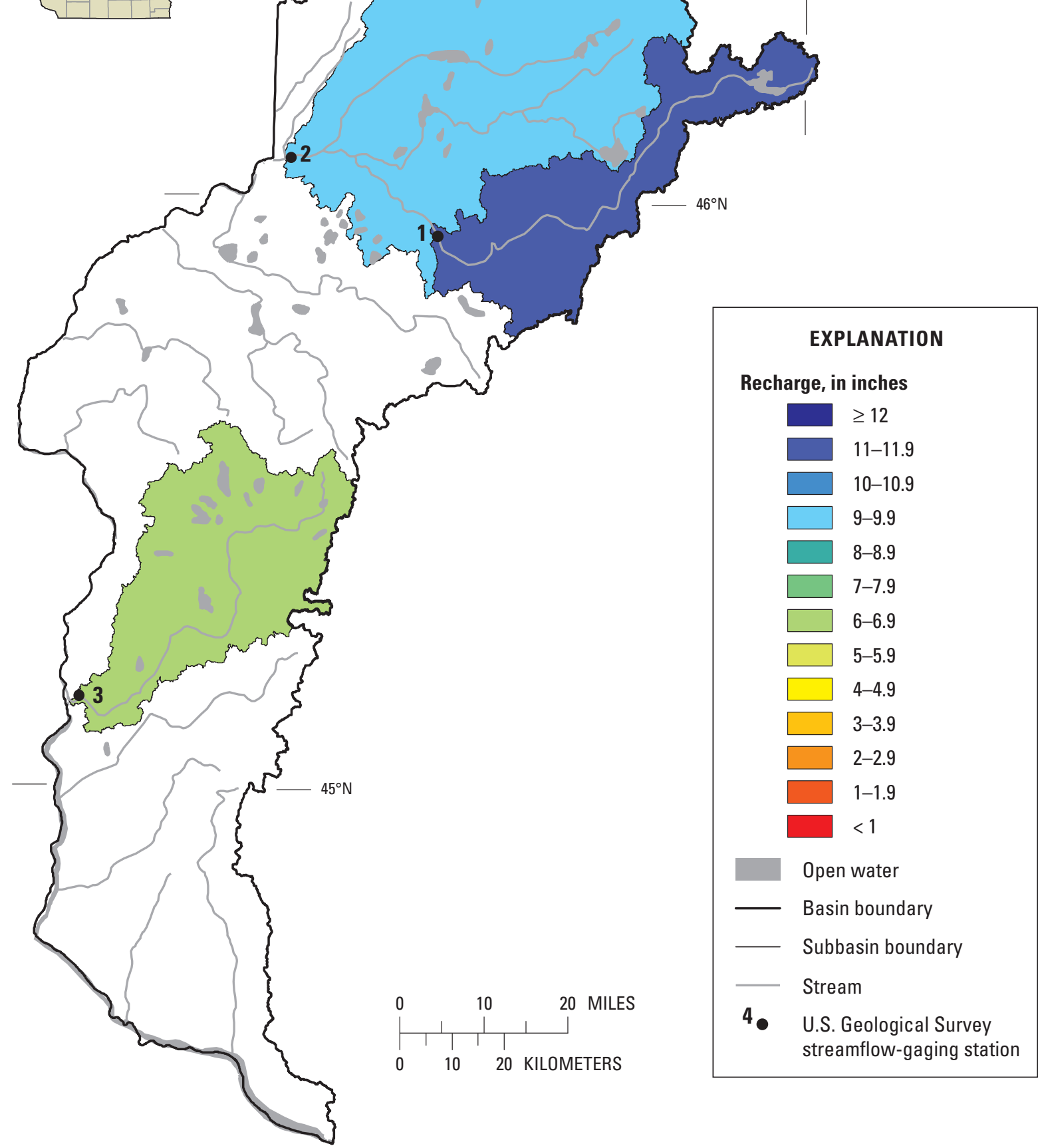

Appendix 2-A. Locations of streamflow-gaging stations in the St. Croix River Basin and spatial distribution of average annual recharge. 
St. Croix

River Basin

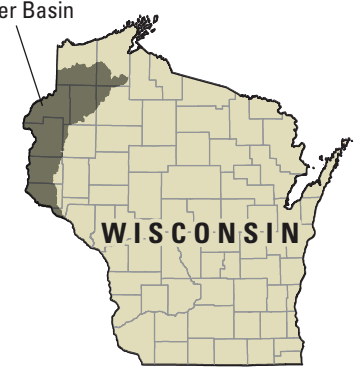

\section{St. Croix River Basin}

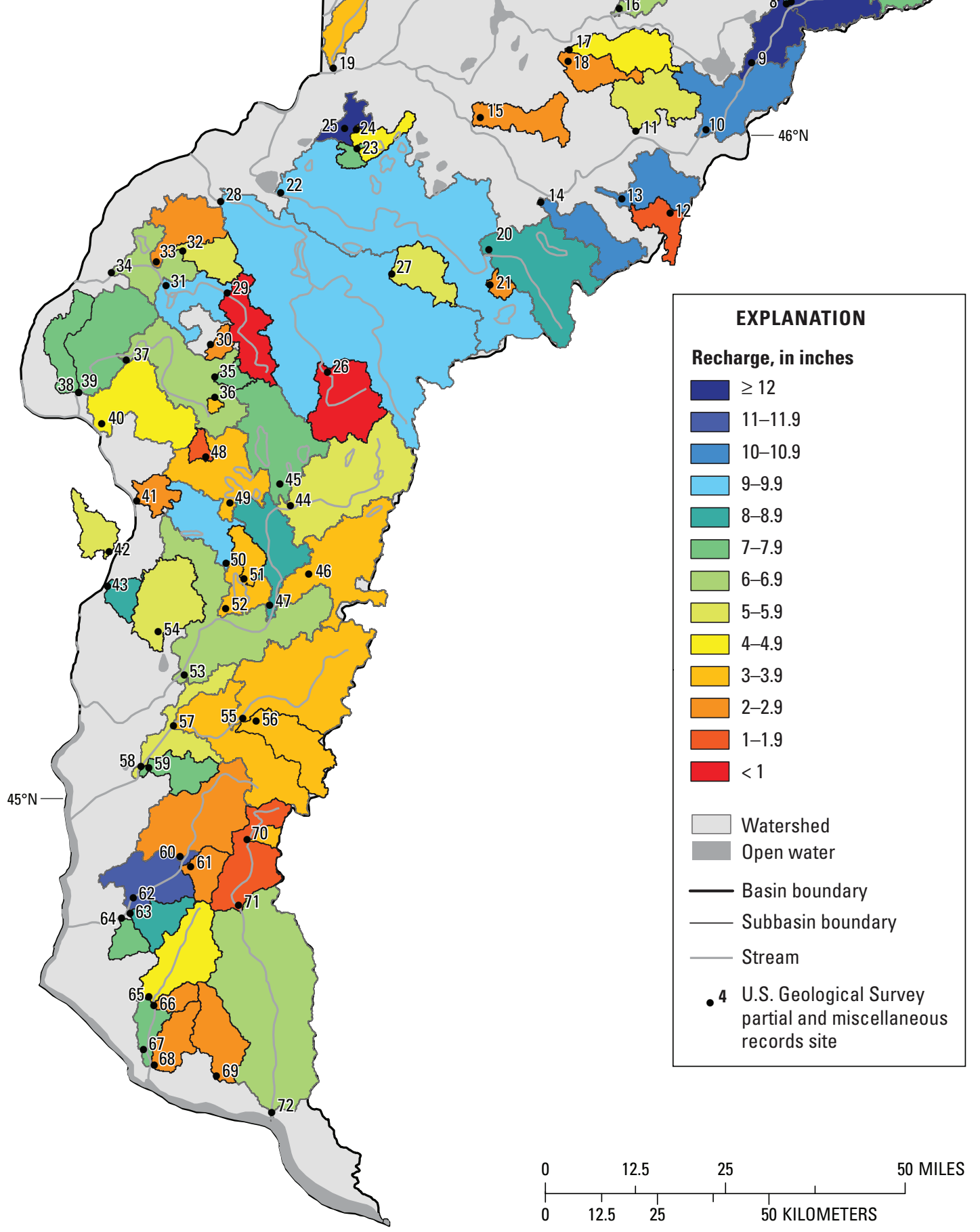

Appendix 2-B. Locations of miscellaneous and partial-record sites in the St. Croix Basin and spatial distribution of estimated average annual recharge. 
Appendix 2C. Average annual base flow and corresponding recharge, 1970-99, for streamflow-streamflow-gaging stations in the St. Croix River Basin.

[USGS, U.S. Geological Survey; ID, identification number; mi², square mile; fts/s, cubic foot per second; BFI, Baseflow Index; ex rec, extended record; R, River; Bk, Brook; Br, Branch; Fk, Fork; Ck, Creek; Tr, Tributary; nr, near; N, North; S, South; E, East; W, West; --, not determined in historical low-flow reports. See references for full list of low-flow reports.]

\begin{tabular}{|c|c|c|c|c|c|c|c|c|c|c|c|}
\hline $\begin{array}{c}\text { Map } \\
\text { number }\end{array}$ & $\begin{array}{l}\text { USGS } \\
\text { station } \\
\text { ID }\end{array}$ & Station name & $\begin{array}{c}\text { Drainage } \\
\text { area } \\
\left(\mathrm{mi}^{2}\right)\end{array}$ & $\begin{array}{c}\text { Period of } \\
\text { analysis } \\
\text { (water years') }\end{array}$ & $\begin{array}{c}\text { Base flow } \\
\text { method }\end{array}$ & $\begin{array}{l}\text { Base flow } \\
\text { index }\end{array}$ & $\begin{array}{c}\text { Base flow } \\
\qquad\left(\mathrm{ft}^{3} / \mathrm{s}\right)\end{array}$ & $\begin{array}{c}\text { Recharge } \\
\text { (inches) }\end{array}$ & $\begin{array}{c}\text { Total } \\
\text { stream- } \\
\text { flow } \\
\left(\mathrm{ft}^{3} / \mathrm{s}\right)\end{array}$ & $\begin{array}{c}\text { Total } \\
\text { streamflow } \\
\text { (inches) }\end{array}$ & $\begin{array}{l}{ }^{2} 090 \\
\left(\mathrm{ft}^{3} / \mathrm{s}\right)\end{array}$ \\
\hline 1 & 05332500 & Namekagon R nr Trego & 488 & 1988-1998 & BFI & -- & 400 & 11.1 & -- & -- & 270 \\
\hline 2 & 05333500 & St. Croix R nr Danbury & 1580 & 1970-1999 & BFI & 0.8000 & 1,100 & 9.5 & 1,400 & 11.9 & 780 \\
\hline 3 & 05341500 & Apple R nr Somerset & 579 & 1970-1999 & BFI & .7150 & 290 & 6.7 & 400 & 9.3 & 190 \\
\hline
\end{tabular}

${ }^{1}$ Water year is a 12-month period from October 1 through September 30, and is designated by the calendar year in which it ends (for example, the 2004 water year occurred October 1, 2003 through September 30, 2004)

${ }^{2}$ Discharge that is exceeded 90 percent of the time. 
Appendix 2D. Average annual base flow and corresponding recharge, 1970-99, for partial-record stations in the St. Croix River Basin.

[USGS, U.S. Geological Survey; ID, identification number; $\mathrm{dd}^{\circ} \mathrm{mm}$ 'ss", degrees, minutes, seconds; $\mathrm{mi}^{2}$, square mile; $\mathrm{ft}^{3} / \mathrm{s} / \mathrm{mi}^{2}$, cubic foot per second per square mile; $\mathrm{ft}^{3} / \mathrm{s}$, cubic foot per second; equation, statewide regression equation; relation, established relation line with index station; R, River; Bk, Brook; Br, Branch; Fk, Fork; Ck, Creek; Tr, Tributary; nr, near; N, North; S, South; E, East; W, West; ,-- not determined in historical low-flow reports. See references for full list of low-flow reports.]

\begin{tabular}{|c|c|c|c|c|c|c|c|c|c|c|}
\hline $\begin{array}{c}\text { Map } \\
\text { number }\end{array}$ & $\begin{array}{c}\text { USGS } \\
\text { station ID }\end{array}$ & Station name & $\begin{array}{c}\text { Latitude } \\
\text { (ddºmm'ss") }\end{array}$ & $\begin{array}{c}\text { Longitude } \\
\text { (ddºmm'ss") }\end{array}$ & $\begin{array}{l}\text { Index } \\
\text { station }^{1}\end{array}$ & $\begin{array}{c}\text { Drainage } \\
\text { area } \\
\left(\mathrm{mi}^{2}\right)\end{array}$ & $\begin{array}{l}\text { Base flow } \\
\text { factor } \\
\left(\mathrm{ft}^{3} / \mathrm{s} / \mathrm{mi}^{2}\right)\end{array}$ & $\begin{array}{c}\text { Base flow } \\
\text { method }\end{array}$ & $\begin{array}{c}\text { Base flow } \\
\quad\left(\mathrm{ft}^{3} / \mathrm{s}\right)\end{array}$ & $\begin{array}{c}\text { Recharge } \\
\text { (inches) }\end{array}$ \\
\hline 1 & 05331582 & St Croix R nr Solon Springs & $46^{\circ} 18^{\prime} 24^{\prime \prime}$ & $91^{\circ} 47^{\prime} 16^{\prime \prime}$ & 05333500 & 34.8 & -- & relation & 23.8 & 9.3 \\
\hline 2 & 05331590 & Lower Ox Ck nr Gordon & $46^{\circ} 17^{\prime} 40^{\prime \prime}$ & $91^{\circ} 44^{\prime} 13^{\prime \prime}$ & 05333500 & 100 & 0.1700 & relation & 18.7 & 2.5 \\
\hline 3 & 05331603 & Eau Claire $\mathrm{R}$ at Gordon & $46^{\circ} 15^{\prime} 09^{\prime \prime}$ & $91^{\circ} 47^{\prime} 57^{\prime \prime}$ & 05333500 & 140 & -- & relation & 103.0 & 10.0 \\
\hline 4 & 05331700 & Moose R nr Solon Springs & $46^{\circ} 17^{\prime} 10^{\prime \prime}$ & $91^{\circ} 57^{\prime} 10^{\prime \prime}$ & 05333500 & 47.8 & .0220 & relation & 14.0 & 4.0 \\
\hline 5 & 05331795 & Castle Ck nr Clam Lk & $46^{\circ} 13^{\prime} 13^{\prime \prime}$ & $91^{\circ} 02^{\prime} 28^{\prime \prime}$ & 05332500 & 13.1 & .1448 & equation & 4.6 & 4.7 \\
\hline 6 & 05331801 & Namekegon R nr Cable & $46^{\circ} 13^{\prime} 26^{\prime \prime}$ & $91^{\circ} 08^{\prime} 52^{\prime \prime}$ & 05332500 & 46.8 & .2687 & equation & 23.1 & 6.7 \\
\hline 7 & 05331820 & Namekagon R nr Cable & $46^{\circ} 11^{\prime} 18^{\prime \prime}$ & $91^{\circ} 19^{\prime} 06^{\prime \prime}$ & 05332500 & 98.8 & .5196 & equation & 69.9 & 9.6 \\
\hline 8 & 05331830 & Big Bk nr Cable & $46^{\circ} 11^{\prime} 06^{\prime \prime}$ & $91^{\circ} 19^{\prime} 39^{\prime \prime}$ & 05332500 & 25 & .5102 & equation & 17.0 & 9.3 \\
\hline 9 & 05331850 & Namekagon R nr Seeley & $46^{\circ} 05^{\prime} 57^{\prime \prime}$ & $91^{\circ} 23^{\prime} 52^{\prime \prime}$ & 05332500 & 157 & .6804 & equation & 129.0 & 11.2 \\
\hline 10 & 05331870 & Namekagon R at Hayward & $46^{\circ} 00^{\prime} 07^{\prime \prime}$ & $91^{\circ} 29^{\prime} 24^{\prime \prime}$ & 05332500 & 206 & .5380 & relation & 167.0 & 11.0 \\
\hline 11 & 05331900 & Chippanazie R at Stanberry & $45^{\circ} 59^{\prime} 50^{\prime \prime}$ & $91^{\circ} 38^{\prime} 05^{\prime \prime}$ & 05333500 & 34.4 & .2110 & relation & 14.3 & 5.6 \\
\hline 12 & 05331945 & Godfrey Ck nr Stone Lk & $45^{\circ} 52^{\prime} 52^{\prime \prime}$ & $91^{\circ} 33^{\prime} 40^{\prime \prime}$ & 05333500 & 7.45 & .2500 & relation & .8 & 1.5 \\
\hline 13 & 05331950 & Bean Bk nr Spring Bk & $45^{\circ} 54^{\prime} 00^{\prime \prime}$ & $91^{\circ} 39^{\prime} 40^{\prime \prime}$ & 05333500 & 45.5 & .7620 & relation & 33.5 & 10.0 \\
\hline 14 & 05332100 & Potato $\mathrm{Ck}$ at Trego & $45^{\circ} 53^{\prime} 32^{\prime \prime}$ & $91^{\circ} 49^{\prime} 39^{\prime \prime}$ & 05333500 & 30.4 & .5340 & relation & 22.9 & 10.2 \\
\hline 15 & 05332700 & Stuntz Ck nr Minong & $46^{\circ} 00^{\prime} 45^{\prime \prime}$ & $91^{\circ} 57^{\prime} 25^{\prime \prime}$ & 05333500 & 21.1 & .0980 & relation & 4.3 & 2.8 \\
\hline 16 & 05333060 & Ounce R nr Gordon & $46^{\circ} 10^{\prime} 25^{\prime \prime}$ & $91^{\circ} 40^{\prime} 30^{\prime \prime}$ & 05333500 & 43.6 & .2630 & relation & 21.2 & 6.6 \\
\hline 17 & 05333080 & Frog Ck nr Minong & $46^{\circ} 06^{\prime} 45^{\prime \prime}$ & $91^{\circ} 46^{\prime} 35^{\prime \prime}$ & 05333500 & 29.4 & .0940 & relation & 9.1 & 4.2 \\
\hline 18 & 05333100 & Little Frog Ck nr Minong & $46^{\circ} 05^{\prime} 48^{\prime \prime}$ & $91^{\circ} 46^{\prime} 39^{\prime \prime}$ & 05333500 & 16.6 & .0570 & relation & 2.6 & 2.1 \\
\hline 19 & 05333510 & Chases Bk nr Danbury & $46^{\circ} 04^{\prime} 40^{\prime \prime}$ & $92^{\circ} 15^{\prime} 50^{\prime \prime}$ & 05333500 & 37.7 & .0670 & relation & 9.2 & 3.3 \\
\hline 20 & 05333996 & Yellow R nr Spooner & $45^{\circ} 49^{\prime} 20^{\prime \prime}$ & $91^{\circ} 55^{\prime} 58^{\prime \prime}$ & 05333500 & 74.3 & .4270 & relation & 44.5 & 8.1 \\
\hline 21 & 05334110 & Sawyer Ck Tr nr Shell Lk & $45^{\circ} 46^{\prime} 17^{\prime \prime}$ & $91^{\circ} 55^{\prime} 44^{\prime \prime}$ & 05332500 & 29.6 & .1560 & relation & 5.7 & 2.6 \\
\hline 22 & 05334500 & Yellow R at Webster & $45^{\circ} 53^{\prime} 45^{\prime \prime}$ & $92^{\circ} 21^{\prime} 53^{\prime \prime}$ & 05333500 & 284 & .4920 & relation & 180.0 & 8.6 \\
\hline 23 & 05335008 & Spring Ck nr Danbury & $45^{\circ} 57^{\prime} 44^{\prime \prime}$ & $92^{\circ} 12^{\prime} 35^{\prime \prime}$ & 05333500 & 5.44 & -- & relation & 2.9 & 7.2 \\
\hline 24 & 05335010 & Loon Ck nr Danbury & $45^{\circ} 59^{\prime} 24^{\prime \prime}$ & $92^{\circ} 12^{\prime} 43^{\prime \prime}$ & 05333500 & 17.6 & -- & relation & 6.6 & 5.1 \\
\hline 25 & 05335017 & Loon Ck nr Danbury & $45^{\circ} 59^{\prime} 28^{\prime \prime}$ & $92^{\circ} 14^{\prime} 13^{\prime \prime}$ & 05333500 & 27.7 & -- & relation & 17.7 & 8.7 \\
\hline 26 & 05335300 & Clam R nr Frederic & $45^{\circ} 38^{\prime} 23^{\prime \prime}$ & $92^{\circ} 15^{\prime} 29^{\prime \prime}$ & 05332500 & 41.6 & .0210 & relation & 1.5 & 0.5 \\
\hline 27 & 05335380 & Bashaw Bk nr Shell Lk & $45^{\circ} 47^{\prime} 02^{\prime \prime}$ & $92^{\circ} 07^{\prime} 51^{\prime \prime}$ & 05368000 & 26.6 & .2720 & relation & 10.0 & 5.1 \\
\hline
\end{tabular}

${ }^{1}$ Low-flow discharge measurements at partial-record stations were compared to one or more nearby USGS gaging stations to establish a relation between measured discharge and concurrent daily discharge. The nearby gaging station that provided the best relation with the partial-record station was chosen as the index station and was associated with nearby miscellaneous measurement sites. 
Appendix 2D. Average annual base flow and corresponding recharge, 1970-99, for partial-record stations in the St. Croix River Basin-Continued.

[USGS, U.S. Geological Survey; ID, identification number; $\mathrm{dd}^{\circ} \mathrm{mm}$ 'ss", degrees, minutes, seconds; $\mathrm{mi}^{2}$, square mile; $\mathrm{ft}^{3} / \mathrm{s} / \mathrm{mi}^{2}$, cubic foot per second per square mile; $\mathrm{ft}^{3} / \mathrm{s}$, cubic foot per second; equation, statewide regression equation; relation, established relation line with index station; R, River; Bk, Brook; Br, Branch; Fk, Fork; Ck, Creek; Tr, Tributary; nr, near; N, North; S, South; E, East; W, West; ,-- not determined in historical low-flow reports. See references for full list of low-flow reports.]

\begin{tabular}{|c|c|c|c|c|c|c|c|c|c|c|}
\hline$\underset{\text { Map }}{\text { number }}$ & $\begin{array}{c}\text { USGS } \\
\text { station ID }\end{array}$ & Station name & $\begin{array}{c}\text { Latitude } \\
\text { (dd }{ }^{\circ} \mathrm{mm} \text { 'ss") }\end{array}$ & $\begin{array}{l}\text { Longitude } \\
\text { (ddºmm'ss") }\end{array}$ & $\begin{array}{l}\text { Index } \\
\text { station }^{1}\end{array}$ & $\begin{array}{c}\text { Drainage } \\
\text { area } \\
\left(\mathrm{mi}^{2}\right)\end{array}$ & $\begin{array}{c}\text { Base flow } \\
\text { factor } \\
\left(\mathrm{ft}^{3} / \mathrm{s} / \mathrm{mi}^{2}\right)\end{array}$ & $\begin{array}{c}\text { Base flow } \\
\text { method }\end{array}$ & $\begin{array}{c}\text { Base flow } \\
\left(\mathrm{ft}^{3} / \mathrm{s}\right)\end{array}$ & $\begin{array}{c}\text { Recharge } \\
\text { (inches) }\end{array}$ \\
\hline 28 & 05335500 & Clam R nr Webster & $45^{\circ} 52^{\prime} 52^{\prime \prime}$ & $92^{\circ} 29^{\prime} 16^{\prime \prime}$ & 05333500 & 361 & 0.3693 & equation & 219.4 & 8.3 \\
\hline 29 & 05338900 & Wood R nr Siren & $45^{\circ} 45^{\prime} 00^{\prime \prime}$ & $92^{\circ} 28^{\prime} 10^{\prime \prime}$ & 05333500 & 29.2 & .0180 & relation & 1.0 & .5 \\
\hline 30 & 05338915 & Wood R Tr nr Frederic & $45^{\circ} 40^{\prime} 27^{\prime \prime}$ & $92^{\circ} 29^{\prime} 58^{\prime \prime}$ & 05341500 & 5.54 & -- & relation & 1.1 & 2.7 \\
\hline 31 & 05338928 & Wood R nr Alpha & $45^{\circ} 45^{\prime} 29^{\prime \prime}$ & $92^{\circ} 35^{\prime} 41^{\prime \prime}$ & 05333500 & 73 & .2770 & relation & 32.9 & 6.1 \\
\hline 32 & 05338940 & N Fk Wood R nr Grantsburg & $45^{\circ} 48^{\prime} 30^{\prime \prime}$ & $92^{\circ} 33^{\prime} 48^{\prime \prime}$ & 05333500 & 17.9 & .2095 & equation & 7.6 & 5.8 \\
\hline 33 & 05338950 & N Fk Wood R nr Grantsburg & $45^{\circ} 47^{\prime} 30^{\prime \prime}$ & $92^{\circ} 37^{\prime} 00^{\prime \prime}$ & 05333500 & 54.8 & .0860 & relation & 9.1 & 2.3 \\
\hline 34 & 05338975 & Wood $\mathrm{R}$ at Grantsburg & $45^{\circ} 46^{\prime} 22^{\prime \prime}$ & $92^{\circ} 42^{\prime} 29^{\prime \prime}$ & 05333500 & 153 & .2360 & relation & 53.7 & 4.8 \\
\hline 35 & 05340300 & Trade R nr Fredric & $45^{\circ} 37^{\prime} 41^{\prime \prime}$ & $92^{\circ} 29^{\prime} 19^{\prime \prime}$ & 05368000 & 6.16 & .2450 & relation & 3.3 & 7.3 \\
\hline 36 & 05340309 & Trade R Tr nr Luck & $45^{\circ} 35^{\prime} 55^{\prime \prime}$ & $92^{\circ} 29^{\prime} 16^{\prime \prime}$ & 05368000 & 2.18 & .1820 & relation & .5 & 3.1 \\
\hline 37 & 05340340 & Trade $\mathrm{R}$ at Trade & $45^{\circ} 38^{\prime} 55^{\prime \prime}$ & $92^{\circ} 40^{\prime} 19^{\prime \prime}$ & 05341500 & 78.3 & .2436 & equation & 37.2 & 6.4 \\
\hline 38 & 05340370 & Trade $\mathrm{R}$ nr Trade $\mathrm{R}$ & $45^{\circ} 35^{\prime} 58^{\prime \prime}$ & $92^{\circ} 46^{\prime} 02^{\prime \prime}$ & 05341500 & 134 & .3255 & equation & 74.8 & 7.6 \\
\hline 39 & 05340385 & Cowan Ck nr Trade R & $45^{\circ} 35^{\prime} 57^{\prime \prime}$ & $92^{\circ} 46^{\prime} 02^{\prime \prime}$ & 05341500 & 23.7 & .3050 & equation & 12.4 & 7.1 \\
\hline 40 & 05340400 & Wolf Ck nr St Croix Falls & $45^{\circ} 33^{\prime} 20^{\prime \prime}$ & $92^{\circ} 43^{\prime} 05^{\prime \prime}$ & 05341500 & 24.9 & .1500 & relation & 7.6 & 4.1 \\
\hline 41 & 05340460 & Big Rock Ck nr St Croix Falls & $45^{\circ} 26^{\prime} 45^{\prime \prime}$ & $92^{\circ} 38^{\prime} 28^{\prime \prime}$ & 05341500 & 10.5 & .0544 & equation & 2.2 & 2.8 \\
\hline 42 & 05340550 & Lawerence Ck at Franconia & $45^{\circ} 22^{\prime} 16^{\prime \prime}$ & $92^{\circ} 41^{\prime} 37^{\prime \prime}$ & 05341500 & 16.2 & .2570 & relation & 6.5 & 5.5 \\
\hline 43 & 053405548 & Trout Bk at Osceola & $45^{\circ} 19^{\prime} 18^{\prime \prime}$ & $92^{\circ} 41^{\prime} 40^{\prime \prime}$ & 05341500 & 10.5 & .2339 & relation & 6.5 & 8.4 \\
\hline 44 & 05340970 & Apple R nr Balsam Lk & $45^{\circ} 26^{\prime} 44^{\prime \prime}$ & $92^{\circ} 19^{\prime} 35^{\prime \prime}$ & 05341500 & 81.7 & .2008 & equation & 35.1 & 5.8 \\
\hline 45 & 05341100 & Fox Ck nr Balsam Lk & $45^{\circ} 28^{\prime} 34^{\prime \prime}$ & $92^{\circ} 20^{\prime} 59^{\prime \prime}$ & 05341500 & 53.9 & .3373 & equation & 30.1 & 7.6 \\
\hline 46 & 05341250 & Beaver Bk nr Joel & $45^{\circ} 20^{\prime} 51^{\prime \prime}$ & $92^{\circ} 17^{\prime} 05^{\prime \prime}$ & 05341500 & 59.8 & .0958 & equation & 17.4 & 3.9 \\
\hline 47 & 05341305 & Apple R nr Amery & $45^{\circ} 18^{\prime} 04^{\prime \prime}$ & $92^{\circ} 21^{\prime} 48^{\prime \prime}$ & 05341500 & 250 & -- & relation & 116.0 & 6.3 \\
\hline 48 & 05341370 & Rice Ck nr Milltown & $45^{\circ} 30^{\prime} 44^{\prime \prime}$ & $92^{\circ} 30^{\prime} 07^{\prime \prime}$ & 05341500 & 3.98 & .0670 & relation & .5 & 1.7 \\
\hline 49 & 05341402 & Balsam Br at Balsam Lk & $45^{\circ} 26^{\prime} 49^{\prime \prime}$ & $92^{\circ} 27^{\prime} 01^{\prime \prime}$ & 05341500 & 52.8 & .1210 & relation & 13.0 & 3.3 \\
\hline 50 & 05341410 & Balsam Br nr Wanderoos & $45^{\circ} 21^{\prime} 35^{\prime \prime}$ & $92^{\circ} 27^{\prime} 13^{\prime \prime}$ & 05341500 & 87.6 & .2101 & equation & 38.6 & 6.0 \\
\hline 51 & 05341418 & Friday Ck nr Deronda & $45^{\circ} 20^{\prime} 20^{\prime \prime}$ & $92^{\circ} 25^{\prime} 02^{\prime \prime}$ & 05341500 & 12.2 & .0945 & equation & 3.4 & 3.8 \\
\hline 52 & 05341426 & Wapogasset Br nr Deronda & $45^{\circ} 17^{\prime} 40^{\prime \prime}$ & $92^{\circ} 27^{\prime} 10^{\prime \prime}$ & 05341500 & 117 & .1696 & equation & 46.4 & 5.4 \\
\hline 53 & 05341437 & Apple R at Star Prairie & $45^{\circ} 11^{\prime} 49^{\prime \prime}$ & $92^{\circ} 31^{\prime} 57^{\prime \prime}$ & 05341500 & 459 & -- & relation & 206.0 & 6.1 \\
\hline
\end{tabular}

${ }^{1}$ Low-flow discharge measurements at partial-record stations were compared to one or more nearby USGS gaging stations to establish a relation between measured discharge and concurrent daily discharge. The nearby gaging station that provided the best relation with the partial-record station was chosen as the index station and was associated with nearby miscellaneous measurement sites. 
Appendix 2D. Average annual base flow and corresponding recharge, 1970-99, for partial-record stations in the St. Croix River Basin-Continued.

[USGS, U.S. Geological Survey; ID, identification number; $\mathrm{dd}^{\circ} \mathrm{mm}$ 'ss", degrees, minutes, seconds; $\mathrm{mi}^{2}$, square mile; $\mathrm{ft}^{3} / \mathrm{s} / \mathrm{mi}^{2}$, cubic foot per second per square mile; $\mathrm{ft}^{3} / \mathrm{s}$, cubic foot per second; equation, statewide regression equation; relation, established relation line with index station; R, River; Bk, Brook; Br, Branch; Fk, Fork; Ck, Creek; Tr, Tributary; nr, near; N, North; S, South; E, East; W, West; --, not determined in historical low-flow reports. See references for full list of low-flow reports.]

\begin{tabular}{|c|c|c|c|c|c|c|c|c|c|c|}
\hline $\begin{array}{c}\text { Map } \\
\text { number }\end{array}$ & $\begin{array}{c}\text { USGS } \\
\text { station ID }\end{array}$ & Station name & $\begin{array}{c}\text { Latitude } \\
\text { (dd }{ }^{\circ} \mathrm{mm} \text { 'ss") }\end{array}$ & $\begin{array}{l}\text { Longitude } \\
\text { (ddºm'ss") }\end{array}$ & $\begin{array}{l}\text { Index } \\
\text { station }{ }^{1}\end{array}$ & $\begin{array}{c}\text { Drainage } \\
\text { area } \\
\left(\mathrm{mi}^{2}\right)\end{array}$ & $\begin{array}{c}\text { Base flow } \\
\text { factor } \\
\left(\mathrm{ft}^{3} / \mathrm{s} / \mathrm{mi}^{2}\right)\end{array}$ & $\begin{array}{l}\text { Base flow } \\
\text { method }\end{array}$ & $\begin{array}{c}\text { Base flow } \\
\left(\mathrm{ft}^{3} / \mathrm{s}\right)\end{array}$ & $\begin{array}{c}\text { Recharge } \\
\text { (inches) }\end{array}$ \\
\hline 54 & 05341450 & Horse Ck nr Star Prairie & $45^{\circ} 15^{\prime} 30^{\prime \prime}$ & $92^{\circ} 35^{\prime} 19^{\prime \prime}$ & 05341500 & 47.1 & 0.3270 & relation & 17.7 & 5.1 \\
\hline 55 & 05341690 & Willow R nr New Richmond & $45^{\circ} 08^{\prime} 13^{\prime \prime}$ & $92^{\circ} 24^{\prime} 40^{\prime \prime}$ & 05368000 & 87.9 & .1270 & relation & 24.5 & 3.8 \\
\hline 56 & 05341694 & S Fk Willow R nr Cylon & $45^{\circ} 08^{\prime} 01^{\prime \prime}$ & $92^{\circ} 23^{\prime} 00^{\prime \prime}$ & 05368000 & 33.3 & .0870 & equation & 9.1 & 3.7 \\
\hline 57 & 05341699 & Willow R at New Richmond & $45^{\circ} 07^{\prime} 24^{\prime \prime}$ & $92^{\circ} 33^{\prime} 02^{\prime \prime}$ & 05368000 & 196 & .1660 & relation & 55.0 & 3.8 \\
\hline 58 & 05341720 & Willow $\mathrm{R}$ at Boardman & $45^{\circ} 03^{\prime} 51^{\prime \prime}$ & $92^{\circ} 36^{\prime} 52^{\prime \prime}$ & 05368000 & 225 & .0967 & equation & 67.5 & 4.1 \\
\hline 59 & 05341730 & Tenmile Ck at Boardman & $45^{\circ} 03^{\prime} 43^{\prime \prime}$ & $92^{\circ} 35^{\prime} 54^{\prime \prime}$ & 05368000 & 17.5 & .3300 & equation & 9.4 & 7.3 \\
\hline 60 & 05341850 & Kinnickinnic R nr Hammond & $44^{\circ} 56^{\prime} 07^{\prime \prime}$ & $92^{\circ} 31^{\prime} 48^{\prime \prime}$ & 05368000 & 67.3 & .2030 & relation & 11.8 & 2.4 \\
\hline 61 & 05341860 & Parker Ck nr Hammond & $44^{\circ} 55^{\prime} 15^{\prime \prime}$ & $92^{\circ} 30^{\prime} 26^{\prime \prime}$ & 05368000 & 13.9 & .0588 & equation & 3.0 & 3.0 \\
\hline 62 & 05341880 & Kinnickinnic R nr R Falls & $44^{\circ} 52^{\prime} 29^{\prime \prime}$ & $92^{\circ} 37^{\prime} 18^{\prime \prime}$ & 05368000 & 115 & .4190 & relation & 43.7 & 5.2 \\
\hline 63 & 05341889 & S Fk Kinnickinnic R at R Falls & $44^{\circ} 51^{\prime} 06^{\prime \prime}$ & $92^{\circ} 37^{\prime} 36^{\prime \prime}$ & 05368000 & 18.1 & .4571 & equation & 11.6 & 8.7 \\
\hline 64 & 05341920 & Kinnickinnic R Tr nr R Falls & $44^{\circ} 50^{\prime} 36^{\prime \prime}$ & $92^{\circ} 38^{\prime} 38^{\prime \prime}$ & 05368000 & 10.4 & .3549 & equation & 5.8 & 7.5 \\
\hline 65 & 05346300 & Trimbelle $\mathrm{R}$ at Trimbelle & $44^{\circ} 43^{\prime} 55^{\prime \prime}$ & $92^{\circ} 35^{\prime} 00^{\prime \prime}$ & 05368000 & 43.3 & .2530 & relation & 13.1 & 4.1 \\
\hline 66 & 05346400 & Spring Ck nr Ellsworth & $44^{\circ} 43^{\prime} 09^{\prime \prime}$ & $92^{\circ} 34^{\prime} 22^{\prime \prime}$ & 05368000 & 7.93 & .0292 & equation & 1.2 & 2.0 \\
\hline 67 & 05346500 & Trimbelle R nr Diamond Bluff & $44^{\circ} 39^{\prime} 24^{\prime \prime}$ & $92^{\circ} 35^{\prime} 28^{\prime \prime}$ & 05368000 & 63.5 & .3460 & equation & 36.0 & 7.7 \\
\hline 68 & 05346600 & Little Trimbelle R nr Bay City & $44^{\circ} 38^{\prime} 01^{\prime \prime}$ & $92^{\circ} 34^{\prime} 05^{\prime \prime}$ & 05368000 & 19.4 & .1150 & relation & 3.5 & 2.5 \\
\hline 69 & 05355300 & Isabelle Ck nr Bay City & $44^{\circ} 37^{\prime} 15^{\prime \prime}$ & $92^{\circ} 26^{\prime} 33^{\prime \prime}$ & 05368000 & 31.1 & .2320 & relation & 6.1 & 2.7 \\
\hline 70 & 05355304 & Rush R Tr at Baldwin & $44^{\circ} 57^{\prime} 47^{\prime \prime}$ & $92^{\circ} 23^{\prime} 42^{\prime \prime}$ & 05379500 & 4.03 & .0898 & equation & 1.1 & 3.6 \\
\hline 71 & 05355308 & Rush R nr Martell & $44^{\circ} 52^{\prime} 03^{\prime \prime}$ & $92^{\circ} 24^{\prime} 31^{\prime \prime}$ & 05379500 & 47.3 & .0210 & equation & 6.2 & 1.8 \\
\hline 72 & 05355325 & Rush R nr Maiden Rock & $44^{\circ} 34^{\prime} 15^{\prime \prime}$ & $92^{\circ} 19^{\prime} 44^{\prime \prime}$ & 05379500 & 213 & -- & relation & 80.0 & 5.1 \\
\hline
\end{tabular}

${ }^{1}$ Low-flow discharge measurements at partial-record stations were compared to one or more nearby USGS gaging stations to establish a relation between measured discharge and concurrent daily discharge. The nearby gaging station that provided the best relation with the partial-record station was chosen as the index station and was associated with nearby miscellaneous measurement sites. 


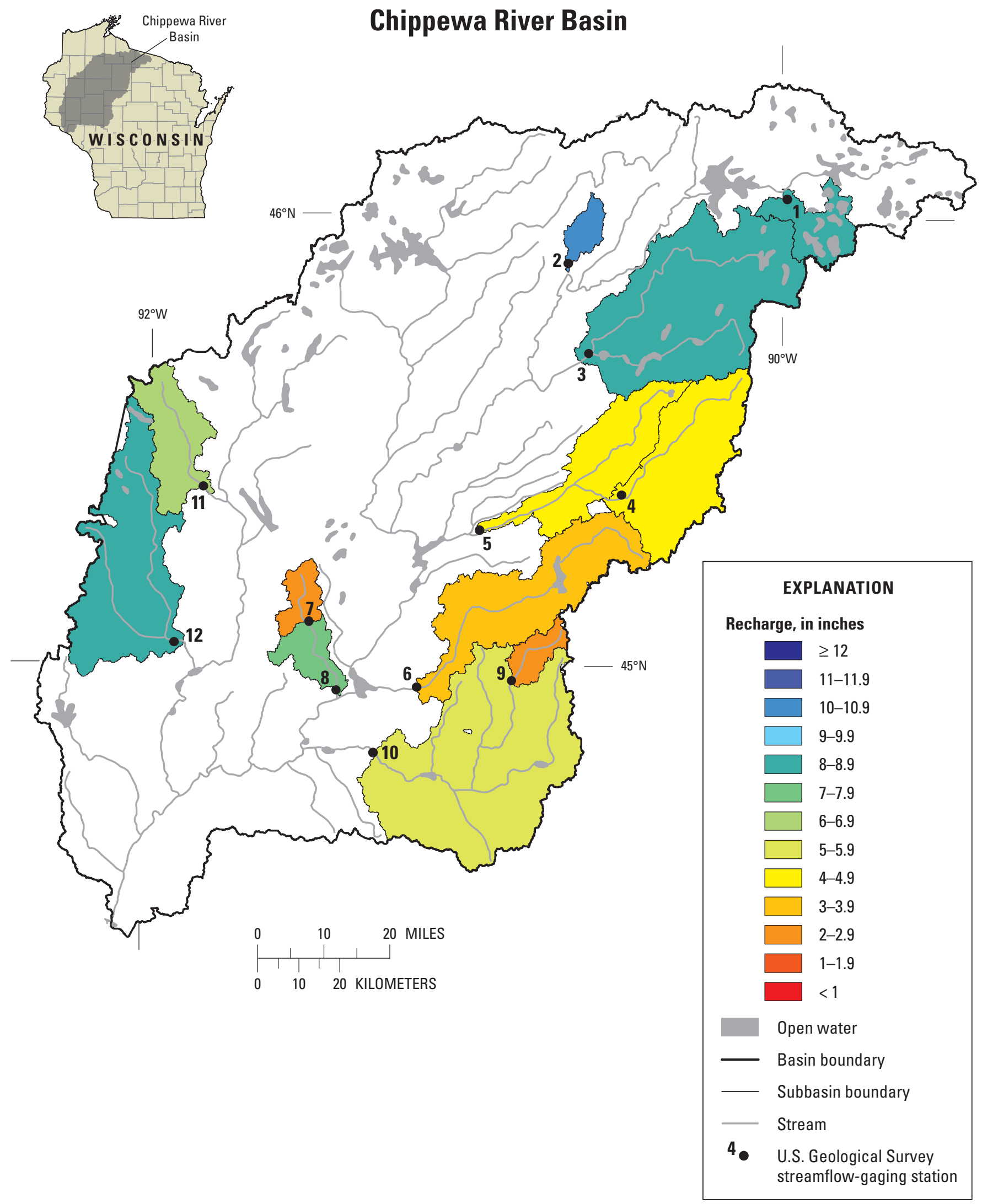

Appendix 3-A, Locations of streamflow-gaging stations in the Chippewa River Basin and spatial distribution of average annual recharge. 


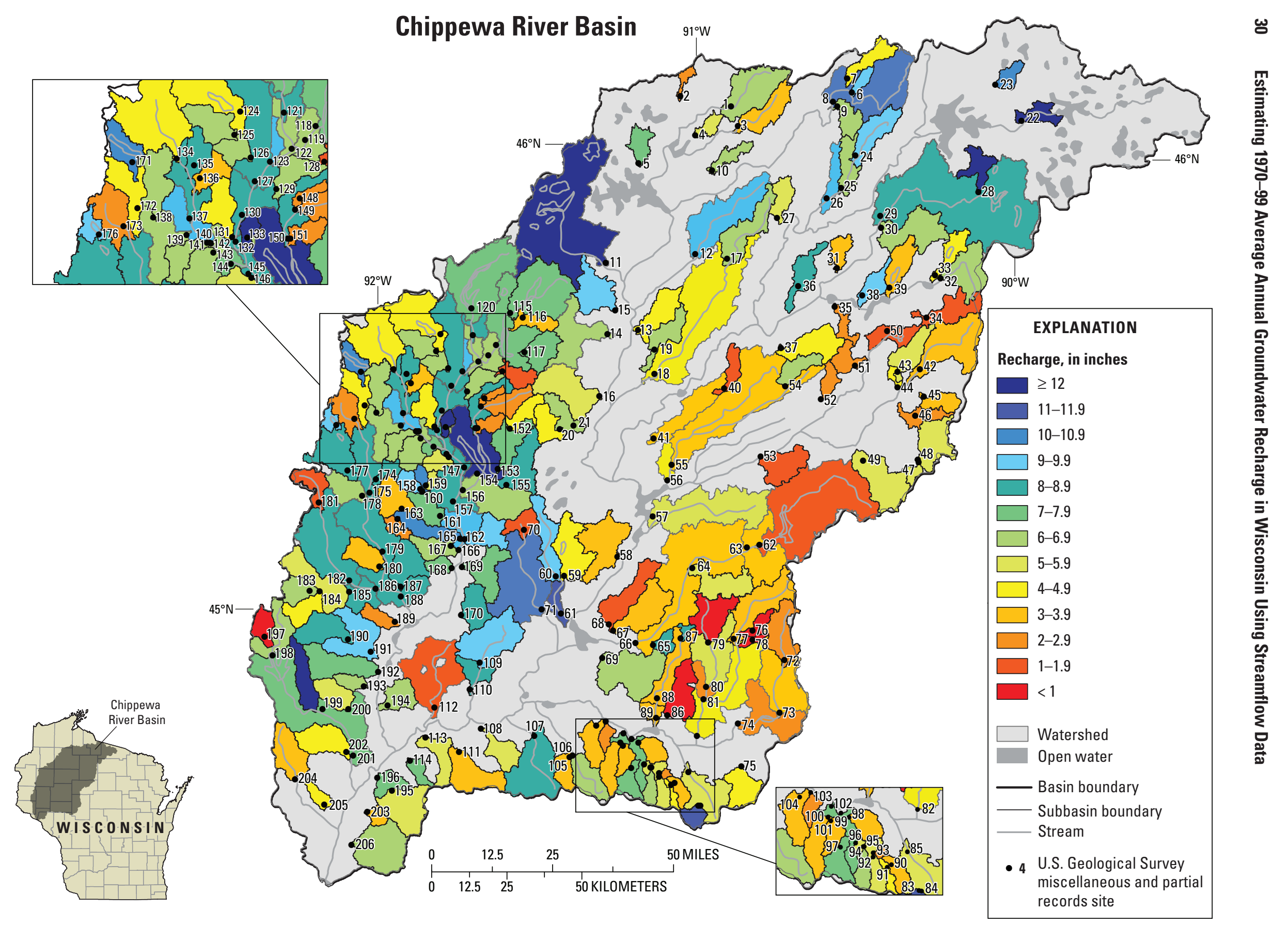

Appendix 3-B. Locations of miscellaneous and partial-record sites in the Chippewa River Basin and spatial distribution of estimated average annual recharge. 
Appendix 3C. Average annual base flow and corresponding recharge, 1970-99, for streamflow-streamflow-gaging stations in the Chippewa River Basin.

[USGS, U.S. Geological Survey; ID, identification number; $\mathrm{mi}^{2}$, square mile; fts/s, cubic foot per second; BFI, Baseflow Index; ex rec, extended record; R, River; Bk, Brook; Br, Branch; Fk, Fork; Ck, Creek; Tr, Tributary; nr, near; N, North; S, South; E, East; W, West; --, not determined in historical low-flow reports. See references for full list of low-flow reports.]

\begin{tabular}{|c|c|c|c|c|c|c|c|c|c|c|c|}
\hline $\begin{array}{c}\text { Map } \\
\text { number }\end{array}$ & $\begin{array}{l}\text { USGS } \\
\text { station } \\
\text { ID }\end{array}$ & Station name & $\begin{array}{c}\text { Drainage } \\
\text { area } \\
\left(\mathrm{mi}^{2}\right)\end{array}$ & $\begin{array}{c}\text { Period of } \\
\text { analysis } \\
\text { (water years }{ }^{1} \text { ) }\end{array}$ & $\begin{array}{c}\text { Base flow } \\
\text { method }\end{array}$ & $\begin{array}{l}\text { Base } \\
\text { flow } \\
\text { index }\end{array}$ & $\begin{array}{l}\text { Base } \\
\text { flow } \\
\left(\mathrm{ft}^{3} / \mathrm{s}\right)\end{array}$ & $\begin{array}{c}\text { Recharge } \\
\text { (inches) }\end{array}$ & $\begin{array}{c}\text { Total } \\
\text { streamflow } \\
\left(\mathrm{ft}^{3} / \mathbf{s}\right)\end{array}$ & $\begin{array}{c}\text { Total } \\
\text { streamflow } \\
\text { (inches) }\end{array}$ & $\begin{array}{l}2090 \\
\left(\mathrm{ft}^{3} / \mathrm{s}\right)\end{array}$ \\
\hline 1 & 05357335 & Bear R nr Manitowish Waters & 81.3 & $1970-1999$ & ex rec & -- & 50 & 8.4 & -- & -- & 21 \\
\hline 2 & 05358300 & Pine Ck nr Oxbow & 38.9 & $1971-1975$ & ex rec & -- & 29 & 10.1 & -- & -- & 14 \\
\hline 3 & 05359500 & S Fork Flambeau R nr Phillips & 609 & $1941-1975$ & ex rec & -- & 390 & 8.7 & -- & -- & 170 \\
\hline 4 & 05361500 & S Fork Jump R nr Ogema & 327 & $1945-1954$ & ex rec & -- & 120 & 5.0 & -- & -- & 39 \\
\hline 5 & 05362000 & Jump R at Sheldon & 576 & 1970-1999 & BFI & 0.3610 & 190 & 4.5 & 540 & 12.8 & 65 \\
\hline 6 & 05364000 & Yellow R at Cadott & 364 & $1943-1961$ & ex rec & -- & 90 & 3.4 & -- & -- & 28 \\
\hline 7 & 05364500 & Duncan $\mathrm{Ck}$ at Bloomer & 50.3 & $1945-1951$ & BFI & -- & 10 & 2.7 & -- & -- & \\
\hline 8 & 05365000 & Duncan Ck at Chippewa Falls & 117 & $1943-1955$ & ex rec & -- & 61 & 7.1 & -- & -- & 25 \\
\hline 9 & 05365707 & North Fork Eau Claire R nr Thorp & 51 & 1970-1999 & ex rec & -- & 8.8 & 2.3 & -- & -- & 1.5 \\
\hline 10 & 05366500 & Eau Claire R nr Fall Ck & 760 & $1943-1955$ & ex rec & -- & 280 & 5.0 & -- & -- & 140 \\
\hline 11 & 053674464 & Yellow $\mathrm{R}$ at Barron & 153 & $1992-1998$ & ex rec & -- & 74 & 6.6 & -- & -- & 50 \\
\hline 12 & 05368000 & Hay R nr Wheeler & 418 & 1970-1999 & BFI & .7600 & 260 & 8.6 & 350 & 11.3 & 189 \\
\hline
\end{tabular}

Water year is a 12-month period from October 1 through September 30, and is designated by the calendar year in which it ends (for example, the 2004 water year occurred October 1, 2003 through September 30, 2004).

${ }^{2}$ Discharge that is exceeded 90 percent of the time. 
[USGS, U.S. Geological Survey; ID, identification number; $\mathrm{dd}^{\circ} \mathrm{mm}$ 'ss", degrees, minutes, seconds; $\mathrm{mi}^{2}$, square mile; $\mathrm{ft}^{3} / \mathrm{s} / \mathrm{mi}^{2}$, cubic foot per second per square mile; $\mathrm{ft}^{3} / \mathrm{s}$, cubic foot per second; equation, statewide regression equation; relation, established relation line with index station; R, River; Bk, Brook; Br, Branch; Fk, Fork; Ck, Creek; Tr, Tributary; nr, near; N, North; S, South; E, East; W, West;

--, not determined in historical low-flow reports. See references for full list of low-flow reports.]

\begin{tabular}{|c|c|c|c|c|c|c|c|c|c|c|}
\hline $\begin{array}{c}\text { Map } \\
\text { number }\end{array}$ & $\begin{array}{c}\text { USGS } \\
\text { station ID }\end{array}$ & Station name & $\begin{array}{c}\text { Latitude } \\
\text { (ddºmm'ss") }\end{array}$ & $\begin{array}{c}\text { Longitude } \\
\text { (ddºmm'ss") }\end{array}$ & $\begin{array}{l}\text { Index } \\
\text { station }^{1}\end{array}$ & $\begin{array}{c}\text { Drainage } \\
\text { area } \\
\left(\mathrm{mi}^{2}\right)\end{array}$ & $\begin{array}{c}\text { Base flow } \\
\text { factor } \\
\left(\mathrm{ft}^{3} / \mathrm{s} / \mathrm{mi}^{2}\right)\end{array}$ & $\begin{array}{c}\text { Base flow } \\
\text { method }\end{array}$ & $\begin{array}{c}\text { Base flow } \\
\qquad\left(\mathrm{ft}^{3} / \mathrm{s}\right)\end{array}$ & $\begin{array}{c}\text { Recharge } \\
\text { (inches) }\end{array}$ \\
\hline 1 & 05355355 & Torch R nr Clam Lk & $46^{\circ} 06^{\prime} 51^{\prime \prime}$ & $90^{\circ} 53^{\prime} 00^{\prime \prime}$ & 05359500 & 43.9 & 0.2229 & equation & 19.7 & 6.1 \\
\hline 2 & 05355361 & Ghost Ck nr New Post & $46^{\circ} 08^{\prime} 07^{\prime \prime}$ & $91^{\circ} 02^{\prime} 34^{\prime \prime}$ & 05332500 & 5.27 & .0384 & equation & .9 & 2.3 \\
\hline 3 & 05355370 & Moose R nr Clam Lk & $46^{\circ} 04^{\prime} 16^{\prime \prime}$ & $90^{\circ} 51^{\prime} 40^{\prime \prime}$ & 05359500 & 26.8 & .0310 & relation & 6.6 & 3.3 \\
\hline 4 & 05355390 & Little Moose R nr New Post & $46^{\circ} 03^{\prime} 02^{\prime \prime}$ & $90^{\circ} 59^{\prime} 39^{\prime \prime}$ & 05359500 & 8.58 & .2258 & equation & 3.7 & 5.9 \\
\hline 5 & 05355420 & Hay Ck nr Hayward & $45^{\circ} 59^{\prime} 19^{\prime \prime}$ & $91^{\circ} 10^{\prime} 12^{\prime \prime}$ & 05332500 & 18.5 & .3080 & relation & 10.3 & 7.6 \\
\hline 6 & 05355522 & Willerth Ck nr Glidden & $46^{\circ} 08^{\prime} 47^{\prime \prime}$ & $90^{\circ} 30^{\prime} 22^{\prime \prime}$ & 04027000 & 4.05 & .5602 & equation & 2.8 & 9.4 \\
\hline 7 & 05355524 & Magee Ck nr Glidden & $46^{\circ} 10^{\prime} 36^{\prime \prime}$ & $90^{\circ} 31^{\prime} 17^{\prime \prime}$ & 04027000 & 14.8 & .1368 & equation & 5.0 & 4.6 \\
\hline 8 & 05355530 & E Fk Chippewa R nr Glidden & $46^{\circ} 07^{\prime} 35^{\prime \prime}$ & $90^{\circ} 33^{\prime} 55^{\prime \prime}$ & 04027000 & 96.7 & .3920 & relation & 66.6 & 9.4 \\
\hline 9 & 05355534 & Schraum Ck nr Glidden & $46^{\circ} 06^{\prime} 58^{\prime \prime}$ & $90^{\circ} 33^{\prime} 05^{\prime \prime}$ & 04027000 & 15.37 & .2715 & equation & 7.5 & 6.6 \\
\hline 10 & 05355585 & Fishtrap Ck nr New Post & $45^{\circ} 58^{\prime} 27^{\prime \prime}$ & $90^{\circ} 56^{\prime} 26^{\prime \prime}$ & 05362000 & 13.1 & .2455 & equation & 6.0 & 6.2 \\
\hline 11 & 05356130 & Couderay R nr Raddison & $45^{\circ} 46^{\prime} 15^{\prime \prime}$ & $91^{\circ} 15^{\prime} 59^{\prime \prime}$ & 05362000 & 187 & -- & relation & 279.0 & ${ }^{2} 20.3$ \\
\hline 12 & 05356170 & Brunet R nr Winter & $45^{\circ} 47^{\prime} 36^{\prime \prime}$ & $90^{\circ} 59^{\prime} 23^{\prime \prime}$ & 05362000 & 66.4 & .3960 & relation & 47.3 & 9.7 \\
\hline 13 & 05356290 & Nail Ck nr Exeland & $45^{\circ} 37^{\prime} 35^{\prime \prime}$ & $91^{\circ} 09^{\prime} 50^{\prime \prime}$ & 05362000 & 26.6 & .1178 & equation & 8.5 & 4.3 \\
\hline 14 & 05356309 & Big Weirgor Ck nr Exeland & $45^{\circ} 36^{\prime} 59^{\prime \prime}$ & $91^{\circ} 15^{\prime} 25^{\prime \prime}$ & 05362000 & 41.7 & .2700 & relation & 20.5 & 6.7 \\
\hline 15 & 05356320 & Little Weirgor Ck at Exeland & $45^{\circ} 40^{\prime} 06^{\prime \prime}$ & $91^{\circ} 14^{\prime} 07^{\prime \prime}$ & 05362000 & 30.6 & .9190 & relation & 20.5 & 9.1 \\
\hline 16 & 05356375 & Devils Ck nr Bruce & $45^{\circ} 28^{\prime} 56^{\prime \prime}$ & $91^{\circ} 16^{\prime} 48^{\prime \prime}$ & 05362000 & 44.1 & .1803 & equation & 17.7 & 5.5 \\
\hline 17 & 05356400 & Thornapple R nr Winter & $45^{\circ} 47^{\prime} 02^{\prime \prime}$ & $90^{\circ} 53^{\prime} 23^{\prime \prime}$ & 05362000 & 30.9 & .2140 & relation & 14.4 & 6.3 \\
\hline 18 & 05356443 & Thornapple R nr Ladysmith & $45^{\circ} 31^{\prime} 52^{\prime \prime}$ & $91^{\circ} 06^{\prime} 40^{\prime \prime}$ & 05362000 & 159 & .1499 & equation & 59.5 & 5.1 \\
\hline 19 & 05356450 & Little Thornapple R nr Ladysmith & $45^{\circ} 35^{\prime} 05^{\prime \prime}$ & $91^{\circ} 06^{\prime} 43^{\prime \prime}$ & 05362000 & 16.3 & .1680 & relation & 8.3 & 6.9 \\
\hline 20 & 05356650 & Soft Maple Ck at Weyerhauser & $45^{\circ} 24^{\prime} 30^{\prime \prime}$ & $91^{\circ} 23^{\prime} 59^{\prime \prime}$ & 05362000 & 15.9 & .2110 & relation & 5.0 & 4.3 \\
\hline 21 & 05356700 & Soft Maple Ck nr Bruce & $45^{\circ} 25^{\prime} 04^{\prime \prime}$ & $91^{\circ} 21^{\prime} 30^{\prime \prime}$ & 05362000 & 32 & .3470 & relation & 13.2 & 5.6 \\
\hline 22 & 05357350 & Lost Ck nr Powell & $46^{\circ} 05^{\prime} 10^{\prime \prime}$ & $89^{\circ} 58^{\prime} 40^{\prime \prime}$ & 05359500 & 15 & .3810 & relation & 15.8 & 14.3 \\
\hline 23 & 05357395 & Little Turtle R at Mercer & $46^{\circ} 09^{\prime} 50^{\prime \prime}$ & $90^{\circ} 03^{\prime} 32^{\prime \prime}$ & 05359500 & 10.4 & .4360 & relation & 8.3 & 10.8 \\
\hline 24 & 05358168 & Butternut $\mathrm{Ck}$ at Butternut & $46^{\circ} 00^{\prime} 37^{\prime \prime}$ & $90^{\circ} 29^{\prime} 41^{\prime \prime}$ & 05359500 & 26.2 & -- & relation & 18.9 & 9.8 \\
\hline
\end{tabular}

${ }^{1}$ Low-flow discharge measurements at partial-record stations were compared to one or more nearby USGS gaging stations to establish a relation between measured discharge and concurrent daily discharge. The nearby gaging station that provided the best relation with the partial-record station was chosen as the index station and was associated with nearby miscellaneous measurement sites.

${ }^{2}$ Mismatch between surface-water and ground-water drainage area. 
Appendix 3D. Average annual baseflow and corresponding recharge, 1970-99, for partial-record stations in the Chippewa River Basin—Continued.

[USGS, U.S. Geological Survey; ID, identification number; $\mathrm{dd}^{\circ} \mathrm{mm}$ 'ss", degrees, minutes, seconds; $\mathrm{mi}^{2}$, square mile; $\mathrm{ft}^{3} / \mathrm{s} / \mathrm{mi}^{2}$, cubic foot per second per square mile; $\mathrm{ft}^{3} / \mathrm{s}$, cubic foot per second; equation, statewide regression equation; relation, established relation line with index station; R, River; Bk, Brook; Br, Branch; Fk, Fork; Ck, Creek; Tr, Tributary; nr, near; N, North; S, South; E, East; W, West; --, not determined in historical low-flow reports. See references for full list of low-flow reports.]

\begin{tabular}{|c|c|c|c|c|c|c|c|c|c|c|}
\hline $\begin{array}{c}\text { Map } \\
\text { number }\end{array}$ & $\begin{array}{c}\text { USGS } \\
\text { station ID }\end{array}$ & Station name & $\begin{array}{c}\text { Latitude } \\
\text { (dd }{ }^{\circ} \mathrm{mm} \text { 'ss") }\end{array}$ & $\begin{array}{c}\text { Longitude } \\
\text { (dd }{ }^{\circ} \mathrm{mm} \text { 'ss") }\end{array}$ & $\begin{array}{l}\text { Index } \\
\text { station }^{1}\end{array}$ & $\begin{array}{c}\text { Drainage } \\
\text { area } \\
\left(\mathrm{mi}^{2}\right)\end{array}$ & $\begin{array}{c}\text { Base flow } \\
\text { factor } \\
\left(\mathrm{ft}^{3} / \mathrm{s} / \mathrm{mi}^{2}\right)\end{array}$ & $\begin{array}{c}\text { Base flow } \\
\text { method }\end{array}$ & $\begin{array}{c}\text { Base flow } \\
\quad\left(\mathrm{ft}^{3} / \mathrm{s}\right)\end{array}$ & $\begin{array}{c}\text { Recharge } \\
\text { (inches) }\end{array}$ \\
\hline 25 & 05358190 & Flambeau R nr Park Falls & $45^{\circ} 56^{\prime} 20^{\prime \prime}$ & $90^{\circ} 32^{\prime} 18^{\prime \prime}$ & 05359500 & 47.6 & 0.2529 & equation & 22.8 & 6.5 \\
\hline 26 & 05358220 & Butternut Ck nr Park Falls & $45^{\circ} 55^{\prime} 00^{\prime \prime}$ & $90^{\circ} 35^{\prime} 01^{\prime \prime}$ & 05359500 & 60.9 & .5209 & equation & 42.7 & 9.5 \\
\hline 27 & 05358337 & Log Ck nr Oxbo & $45^{\circ} 52^{\prime} 24^{\prime \prime}$ & $90^{\circ} 44^{\prime} 10^{\prime \prime}$ & 05359500 & 14.8 & .2127 & equation & 6.3 & 5.8 \\
\hline 28 & 05359100 & Springstead Ck nr Park Falls & $45^{\circ} 55^{\prime} 55^{\prime \prime}$ & $90^{\circ} 06^{\prime} 40^{\prime \prime}$ & 05359500 & 17 & .3060 & relation & 16.6 & 13.3 \\
\hline 29 & 05359215 & S Fk Flambeau R at Fifield & $45^{\circ} 52^{\prime} 48^{\prime \prime}$ & $90^{\circ} 24^{\prime} 56^{\prime \prime}$ & 05359500 & 241 & -- & relation & 158.0 & 8.9 \\
\hline 30 & 05359249 & Sailor Ck nr Fifield & $45^{\circ} 51^{\prime} 13^{\prime \prime}$ & $90^{\circ} 24^{\prime} 45^{\prime \prime}$ & 05359500 & 35.3 & .2531 & equation & 16.8 & 6.5 \\
\hline 31 & 05359277 & Smith Ck nr Lugerville & $45^{\circ} 45^{\prime} 57^{\prime \prime}$ & $90^{\circ} 32^{\prime} 58^{\prime \prime}$ & 05359500 & 11.4 & .0637 & equation & 2.6 & 3.1 \\
\hline 32 & 05359350 & Elk R nr Phillips & $45^{\circ} 44^{\prime} 40^{\prime \prime}$ & $90^{\circ} 13^{\prime} 40^{\prime \prime}$ & 05359500 & 34 & .2130 & relation & 15.8 & 6.3 \\
\hline 33 & 05359355 & Popple Ck nr Phillips & $45^{\circ} 45^{\prime} 05^{\prime \prime}$ & $90^{\circ} 14^{\prime} 46^{\prime \prime}$ & 05359500 & 18.3 & .1072 & equation & 5.5 & 4.1 \\
\hline 34 & 053593626 & Little Elk R nr Phillips & $45^{\circ} 39^{\prime} 31^{\prime \prime}$ & $90^{\circ} 16^{\prime} 17^{\prime \prime}$ & 05362000 & 23.7 & .0198 & equation & 3.0 & 1.7 \\
\hline 35 & 05359447 & Carpenter Ck nr Phillips & $45^{\circ} 40^{\prime} 54^{\prime \prime}$ & $90^{\circ} 33^{\prime} 19^{\prime \prime}$ & 05359500 & 12.4 & .0410 & equation & 2.2 & 2.5 \\
\hline 36 & 05359600 & Price Ck nr Phillips & $45^{\circ} 43^{\prime} 33^{\prime \prime}$ & $90^{\circ} 40^{\prime} 12^{\prime \prime}$ & 05359500 & 16.9 & .4560 & relation & 10.9 & 8.8 \\
\hline 37 & 05359700 & Skinner Ck nr Hawkins & $45^{\circ} 35^{\prime} 29^{\prime \prime}$ & $90^{\circ} 43^{\prime} 14^{\prime \prime}$ & 05362000 & 29.8 & .0520 & relation & 9.3 & 4.2 \\
\hline 38 & 05359809 & Deer Ck nr Phillips & $45^{\circ} 42^{\prime} 24^{\prime \prime}$ & $90^{\circ} 28^{\prime} 15^{\prime \prime}$ & 05359500 & 12.9 & .5069 & equation & 8.6 & 9.1 \\
\hline 39 & 05359871 & Squaw Ck nr Phillips & $45^{\circ} 43^{\prime} 25^{\prime \prime}$ & $90^{\circ} 23^{\prime} 11^{\prime \prime}$ & 05359500 & 23 & .0979 & equation & 6.6 & 3.9 \\
\hline 40 & 05361050 & Deer Tail Ck at Glen Flora & $45^{\circ} 30^{\prime} 10^{\prime \prime}$ & $90^{\circ} 53^{\prime} 41^{\prime \prime}$ & 05362000 & 11.1 & .0269 & equation & 1.6 & 2.0 \\
\hline 41 & 05361100 & Deer Tail Ck nr Ladysmith & $45^{\circ} 23^{\prime} 33^{\prime \prime}$ & $91^{\circ} 06^{\prime} 37^{\prime \prime}$ & 05362000 & 43.9 & .0440 & relation & 9.4 & 2.9 \\
\hline 42 & 05361390 & S Fk Jump R at Prentice & $45^{\circ} 32^{\prime} 51^{\prime \prime}$ & $90^{\circ} 17^{\prime} 30^{\prime \prime}$ & 05362000 & 57.2 & .0320 & relation & 14.2 & 3.4 \\
\hline 43 & 05361400 & Hay Ck nr Prentice & $45^{\circ} 32^{\prime} 32^{\prime \prime}$ & $90^{\circ} 21^{\prime} 37^{\prime \prime}$ & 05362000 & 22.6 & .0500 & relation & 8.6 & 5.2 \\
\hline 44 & 05361405 & Hay Ck nr Prentice & $45^{\circ} 30^{\prime} 31^{\prime \prime}$ & $90^{\circ} 21^{\prime} 35^{\prime \prime}$ & 05362000 & 27.4 & .1300 & equation & 9.2 & 4.6 \\
\hline 45 & 05361424 & Mondeaux Ck nr Ogema & $45^{\circ} 29^{\prime} 18^{\prime \prime}$ & $90^{\circ} 16^{\prime} 46^{\prime \prime}$ & 05362000 & 11.1 & .0685 & equation & 2.6 & 3.2 \\
\hline 46 & 05361438 & Holmes $\mathrm{Ck}$ at Ogema & $45^{\circ} 26^{\prime} 45^{\prime \prime}$ & $90^{\circ} 18^{\prime} 21^{\prime \prime}$ & 05362000 & 10.4 & .0130 & relation & 1.9 & 2.5 \\
\hline 47 & 05361478 & Fisher Ck at Wboro & $45^{\circ} 20^{\prime} 47^{\prime \prime}$ & $90^{\circ} 17^{\prime} 39^{\prime \prime}$ & 05362000 & 5.48 & .2300 & equation & 2.4 & 5.9 \\
\hline 48 & 05361490 & Silver Ck at Wboro & $45^{\circ} 21^{\prime} 02^{\prime \prime}$ & $90^{\circ} 17^{\prime} 49^{\prime \prime}$ & 05362000 & 36.4 & .0740 & relation & 14.2 & 5.3 \\
\hline
\end{tabular}

${ }^{1}$ Low-flow discharge measurements at partial-record stations were compared to one or more nearby USGS gaging stations to establish a relation between measured discharge and concurrent daily discharge. The nearby gaging station that provided the best relation with the partial-record station was chosen as the index station and was associated with nearby miscellaneous measurement sites.

${ }^{2}$ Mismatch between surface-water and ground-water drainage area. 
[USGS, U.S. Geological Survey; ID, identification number; $\mathrm{dd}^{\circ} \mathrm{mm}$ 'ss", degrees, minutes, seconds; $\mathrm{mi}^{2}$, square mile; $\mathrm{ft}^{3} / \mathrm{s} / \mathrm{mi}^{2}$, cubic foot per second per square mile; $\mathrm{ft}^{3} / \mathrm{s}$, cubic foot per second; equation, statewide regression equation; relation, established relation line with index station; R, River; Bk, Brook; Br, Branch; Fk, Fork; Ck, Creek; Tr, Tributary; nr, near; N, North; S, South; E, East; W, West;

--, not determined in historical low-flow reports. See references for full list of low-flow reports.]

\begin{tabular}{|c|c|c|c|c|c|c|c|c|c|c|}
\hline $\begin{array}{c}\text { Map } \\
\text { number }\end{array}$ & $\begin{array}{c}\text { USGS } \\
\text { station ID }\end{array}$ & Station name & $\begin{array}{c}\text { Latitude } \\
\text { (dd }{ }^{\circ} \mathrm{mm} \text { 'ss") }\end{array}$ & $\begin{array}{c}\text { Longitude } \\
\text { (dd }{ }^{\circ} \mathrm{mm} \text { 'ss") }\end{array}$ & $\begin{array}{l}\text { Index } \\
\text { station }^{1}\end{array}$ & $\begin{array}{c}\text { Drainage } \\
\text { area } \\
\left(\mathrm{mi}^{2}\right)\end{array}$ & $\begin{array}{c}\text { Base flow } \\
\text { factor } \\
\left(\mathrm{ft}^{3} / \mathrm{s} / \mathrm{mi}^{2}\right)\end{array}$ & $\begin{array}{l}\text { Base flow } \\
\text { method }\end{array}$ & $\begin{array}{c}\text { Base flow } \\
\qquad\left(\mathrm{ft}^{3} / \mathrm{s}\right)\end{array}$ & $\begin{array}{c}\text { Recharge } \\
\text { (inches) }\end{array}$ \\
\hline 49 & 053614983 & Mondeaux Ck nr Wboro & $45^{\circ} 20^{\prime} 53^{\prime \prime}$ & $90^{\circ} 27^{\prime} 52^{\prime \prime}$ & 05362000 & 39.2 & 0.1713 & equation & 15.3 & 5.3 \\
\hline 50 & 05361600 & N Fk Jump R nr Phillips & $45^{\circ} 37^{\prime} 45^{\prime \prime}$ & $90^{\circ} 23^{\prime} 32^{\prime \prime}$ & 05362000 & 10.5 & .0252 & equation & 1.5 & 1.9 \\
\hline 51 & 05361606 & Needle Ck nr Catawba & $45^{\circ} 33^{\prime} 15^{\prime \prime}$ & $90^{\circ} 29^{\prime} 32^{\prime \prime}$ & 05362000 & 8.9 & .0366 & equation & 1.5 & 2.3 \\
\hline 52 & 05361828 & Web Ck nr Catawba & $45^{\circ} 28^{\prime} 52^{\prime \prime}$ & $90^{\circ} 35^{\prime} 48^{\prime \prime}$ & 05362000 & 9.5 & .0437 & equation & 1.8 & 2.5 \\
\hline 53 & 05361920 & Levitt Ck at Jump R & $45^{\circ} 21^{\prime} 22^{\prime \prime}$ & $90^{\circ} 46^{\prime} 48^{\prime \prime}$ & 05362000 & 28.4 & .0136 & equation & 2.9 & 1.4 \\
\hline 54 & 05362472 & S Fk Main Ck at Hawkins & $45^{\circ} 30^{\prime} 32^{\prime \prime}$ & $90^{\circ} 42^{\prime} 25^{\prime \prime}$ & 05362000 & 16.2 & .0550 & relation & 7.5 & 6.3 \\
\hline 55 & 05362485 & Main Ck nr Sheldon & $45^{\circ} 20^{\prime} 07^{\prime \prime}$ & $91^{\circ} 03^{\prime} 16^{\prime \prime}$ & 05362000 & 138 & .0771 & equation & 36.4 & 3.6 \\
\hline 56 & 05362487 & Main Ck nr Coranth & $45^{\circ} 18^{\prime} 09^{\prime \prime}$ & $91^{\circ} 03^{\prime} 59^{\prime \prime}$ & 05362000 & 149 & .0994 & equation & 45.0 & 4.1 \\
\hline 57 & 05363000 & Fisher R at Holcombe & $45^{\circ} 13^{\prime} 20^{\prime \prime}$ & $91^{\circ} 06^{\prime} 35^{\prime \prime}$ & 05381000 & 81.5 & .0390 & relation & 33.0 & 5.5 \\
\hline 58 & 05363110 & N Fk Ck Bob Ck nr Cornell & $45^{\circ} 08^{\prime} 03^{\prime \prime}$ & $91^{\circ} 12^{\prime} 58^{\prime \prime}$ & 05364000 & 42 & .0775 & equation & 10.9 & 3.5 \\
\hline 59 & 05363290 & O'Neil Ck nr Eagleton & $45^{\circ} 05^{\prime} 26^{\prime \prime}$ & $91^{\circ} 22^{\prime} 46^{\prime \prime}$ & 05368000 & 38.6 & .1529 & equation & 14.2 & 5.0 \\
\hline 60 & 05363360 & Mccann Ck nr Eagleton & $45^{\circ} 05^{\prime} 22^{\prime \prime}$ & $91^{\circ} 24^{\prime} 17^{\prime \prime}$ & 05368000 & 29.7 & .5010 & equation & 20.1 & 9.2 \\
\hline 61 & 05363500 & O'Neil Ck nr Chippewa Falls & $45^{\circ} 00^{\prime} 32^{\prime \prime}$ & $91^{\circ} 23^{\prime} 13^{\prime \prime}$ & 05368000 & 78.1 & .3730 & relation & 68.0 & 11.8 \\
\hline 62 & 05363765 & Yellow R nr Gilman & $45^{\circ} 09^{\prime} 47^{\prime \prime}$ & $90^{\circ} 46^{\prime} 54^{\prime \prime}$ & 05364000 & 146 & .0174 & equation & 17.8 & 1.7 \\
\hline 63 & 05363810 & Yellow R at Gilman & $45^{\circ} 09^{\prime} 26^{\prime \prime}$ & $90^{\circ} 49^{\prime} 12^{\prime \prime}$ & 05364000 & 187 & -- & relation & 46.3 & 3.4 \\
\hline 64 & 05363943 & Otter $\mathrm{Ck}$ at Huron & $45^{\circ} 06^{\prime} 45^{\prime \prime}$ & $90^{\circ} 59^{\prime} 11 "$ & 05364000 & 30.7 & .1818 & equation & 12.3 & 5.4 \\
\hline 65 & 05363990 & Lotz R nr Boyd & $44^{\circ} 56^{\prime} 39^{\prime \prime}$ & $91^{\circ} 06^{\prime} 13^{\prime \prime}$ & 05364000 & 7.66 & .4715 & equation & 4.9 & 8.7 \\
\hline 66 & 05364005 & Yellow R at Cadott & $44^{\circ} 56^{\prime} 54^{\prime \prime}$ & $91^{\circ} 09^{\prime} 29^{\prime \prime}$ & 05364000 & 365 & .0767 & equation & 98.0 & 3.6 \\
\hline 67 & 05364120 & Big Drywood Ck nr Cadott & $44^{\circ} 58^{\prime} 27^{\prime \prime}$ & $91^{\circ} 13^{\prime} 36^{\prime \prime}$ & 05362000 & 33.5 & .1580 & relation & 8.0 & 3.2 \\
\hline 68 & 05364125 & Little Drywood Ck nr Cadott & $44^{\circ} 59^{\prime} 16^{\prime \prime}$ & $91^{\circ} 14^{\prime} 22^{\prime \prime}$ & 05364000 & 33 & .0180 & equation & 4.0 & 1.6 \\
\hline 69 & 05364160 & Paint Ck nr Chippewa Falls & $44^{\circ} 54^{\prime} 52^{\prime \prime}$ & $91^{\circ} 15^{\prime} 27^{\prime \prime}$ & 05381000 & 55.5 & .2620 & relation & 25.1 & 6.1 \\
\hline 70 & 05364260 & Duncan Ck nr New Auburn & $45^{\circ} 11^{\prime} 21^{\prime \prime}$ & $91^{\circ} 30^{\prime} 13^{\prime \prime}$ & 05368000 & 11.5 & .3140 & relation & 1.6 & 1.9 \\
\hline 71 & 05364750 & Duncan $\mathrm{Ck}$ at Tilden & $45^{\circ} 01^{\prime} 06^{\prime \prime}$ & $91^{\circ} 26^{\prime} 43^{\prime \prime}$ & 05368000 & 97.5 & .5384 & equation & 70.2 & 9.8 \\
\hline 72 & 05365570 & S Fk Eau Claire R nr Withee & $44^{\circ} 54^{\prime} 54^{\prime \prime}$ & $90^{\circ} 42^{\prime} 08^{\prime \prime}$ & 05381000 & 28.2 & .0333 & equation & 4.7 & 2.2 \\
\hline
\end{tabular}

${ }^{1}$ Low-flow discharge measurements at partial-record stations were compared to one or more nearby USGS gaging stations to establish a relation between measured discharge and concurrent daily discharge. The nearby gaging station that provided the best relation with the partial-record station was chosen as the index station and was associated with nearby miscellaneous measurement sites.

${ }^{2}$ Mismatch between surface-water and ground-water drainage area. 
Appendix 3D. Average annual baseflow and corresponding recharge, 1970-99, for partial-record stations in the Chippewa River Basin—Continued.

[USGS, U.S. Geological Survey; ID, identification number; $\mathrm{dd}^{\circ} \mathrm{mm}$ 'ss", degrees, minutes, seconds; $\mathrm{mi}^{2}$, square mile; $\mathrm{ft}^{3} / \mathrm{s} / \mathrm{mi}^{2}$, cubic foot per second per square mile; $\mathrm{ft}^{3} / \mathrm{s}$, cubic foot per second; equation, statewide regression equation; relation, established relation line with index station; R, River; Bk, Brook; Br, Branch; Fk, Fork; Ck, Creek; Tr, Tributary; nr, near; N, North; S, South; E, East; W, West; --, not determined in historical low-flow reports. See references for full list of low-flow reports.]

\begin{tabular}{|c|c|c|c|c|c|c|c|c|c|c|}
\hline $\begin{array}{c}\text { Map } \\
\text { number }\end{array}$ & $\begin{array}{c}\text { USGS } \\
\text { station ID }\end{array}$ & Station name & $\begin{array}{c}\text { Latitude } \\
\text { (dd }{ }^{\circ} \mathrm{mm} \text { 'ss") }\end{array}$ & $\begin{array}{c}\text { Longitude } \\
\text { (dd }{ }^{\circ} \mathrm{mm} \text { 'ss") }\end{array}$ & $\begin{array}{l}\text { Index } \\
\text { station }{ }^{1}\end{array}$ & $\begin{array}{c}\text { Drainage } \\
\text { area } \\
\left(\mathrm{mi}^{2}\right)\end{array}$ & $\begin{array}{c}\text { Base flow } \\
\text { factor } \\
\left(\mathrm{ft}^{3} / \mathrm{s} / \mathrm{mi}^{2}\right)\end{array}$ & $\begin{array}{c}\text { Base flow } \\
\text { method }\end{array}$ & $\begin{array}{c}\text { Base flow } \\
\qquad\left(\mathrm{ft}^{3} / \mathrm{s}\right)\end{array}$ & $\begin{array}{c}\text { Recharge } \\
\text { (inches) }\end{array}$ \\
\hline 73 & 05365600 & S Fk Eau Claire R nr Greenwood & $44^{\circ} 47^{\prime} 58^{\prime \prime}$ & $90^{\circ} 42^{\prime} 53^{\prime \prime}$ & 05381000 & 77.6 & 0.0280 & relation & 19.0 & 3.3 \\
\hline 74 & 05365619 & S Fk Eau Claire R nr Thorp & $44^{\circ} 46^{\prime} 33^{\prime \prime}$ & $90^{\circ} 50^{\prime} 29^{\prime \prime}$ & 05381000 & 122 & .0321 & equation & 20.4 & 2.3 \\
\hline 75 & 05365650 & Hay Ck nr Fairchild & $44^{\circ} 41^{\prime} 01^{\prime \prime}$ & $90^{\circ} 49^{\prime} 49^{\prime \prime}$ & 05366500 & 22.5 & .0450 & relation & 7.8 & 4.7 \\
\hline 76 & 05365700 & Goggle-Eye Ck nr Thorp & $44^{\circ} 58^{\prime} 40^{\prime \prime}$ & $90^{\circ} 48^{\prime} 00^{\prime \prime}$ & 05362000 & 6.42 & .0200 & relation & .3 & .7 \\
\hline 77 & 05365710 & N Fk Eau Claire R nr Thorp & $44^{\circ} 57^{\prime} 33^{\prime \prime}$ & $90^{\circ} 51^{\prime} 30^{\prime \prime}$ & 05364000 & 52.9 & -- & relation & 11.6 & 3.0 \\
\hline 78 & 05365720 & Mcgrogan Ck at Thorp & $44^{\circ} 57^{\prime} 28^{\prime \prime}$ & $90^{\circ} 48^{\prime} 01^{\prime \prime}$ & 05381000 & 2.22 & .0050 & equation & .1 & .8 \\
\hline 79 & 05365840 & Wolf R at Stanley & $44^{\circ} 57^{\prime} 06^{\prime \prime}$ & $90^{\circ} 56^{\prime} 05^{\prime \prime}$ & 05381000 & 30.8 & .0006 & equation & .6 & .3 \\
\hline 80 & 05365900 & Wolf R nr Stanley & $44^{\circ} 51^{\prime} 14^{\prime \prime}$ & $90^{\circ} 56^{\prime} 23^{\prime \prime}$ & 05381000 & 71.2 & .0800 & relation & 18.0 & 3.4 \\
\hline 81 & 05365910 & Wolf R nr Stanley & $44^{\circ} 49^{\prime} 40^{\prime \prime}$ & $90^{\circ} 56^{\prime} 53^{\prime \prime}$ & 05381000 & 81.3 & .0490 & equation & 16.8 & 2.8 \\
\hline 82 & 05365928 & N Fk Eau Claire R nr Augusta & $44^{\circ} 44^{\prime} 53^{\prime \prime}$ & $90^{\circ} 58^{\prime} 00^{\prime \prime}$ & 05366500 & 205 & .0735 & equation & 53.2 & 3.5 \\
\hline 83 & 05365954 & Schoolhouse Ck at Fairchild & $44^{\circ} 35^{\prime} 51^{\prime \prime}$ & $90^{\circ} 57^{\prime} 35^{\prime \prime}$ & 05379500 & 6.52 & .2451 & equation & 3.0 & 6.2 \\
\hline 84 & 05365958 & Schoolhouse Ck at Fairchild & $44^{\circ} 64^{\prime} 05^{\prime \prime}$ & $90^{\circ} 57^{\prime} 11 "$ & 05364000 & 9.6 & .8489 & equation & 8.4 & 11.8 \\
\hline 85 & 05365970 & Black Ck nr Fairchild & $44^{\circ} 40^{\prime} 07^{\prime \prime}$ & $90^{\circ} 59^{\prime} 26^{\prime \prime}$ & 05366500 & 43.9 & .2595 & equation & 21.3 & 6.6 \\
\hline 86 & 05366080 & Muskrat Ck nr Augusta & $44^{\circ} 47^{\prime} 30^{\prime \prime}$ & $91^{\circ} 03 ' 28^{\prime \prime}$ & 05366500 & 29.6 & .0062 & equation & 2.0 & .9 \\
\hline 87 & 05366090 & Hay Ck nr Boyd & $44^{\circ} 57^{\prime} 31^{\prime \prime}$ & $91^{\circ} 01^{\prime} 11^{\prime \prime}$ & 05364000 & 2.62 & .5760 & relation & 1.7 & 8.8 \\
\hline 88 & 05366165 & Hay Ck nr Ludington & $44^{\circ} 49^{\prime} 44^{\prime \prime}$ & $91^{\circ} 05^{\prime} 24^{\prime \prime}$ & 05381000 & 31.1 & .0918 & equation & 8.7 & 3.8 \\
\hline 89 & 05366180 & Hay Ck nr Augusta & $44^{\circ} 47^{\prime} 08^{\prime \prime}$ & $91^{\circ} 05^{\prime} 29^{\prime \prime}$ & 05366500 & 39.9 & .0851 & equation & 10.8 & 3.7 \\
\hline 90 & 05366264 & Bridge Ck nr Fairchild & $44^{\circ} 38^{\prime} 43^{\prime \prime}$ & $91^{\circ} 01^{\prime} 57^{\prime \prime}$ & 05379500 & 6.01 & .0972 & equation & 1.7 & 3.8 \\
\hline 91 & 05366267 & Travis Ck nr Fairchild & $44^{\circ} 38^{\prime} 25^{\prime \prime}$ & $91^{\circ} 02^{\prime} 34^{\prime \prime}$ & 05379500 & 3.53 & .1537 & equation & 1.2 & 4.8 \\
\hline 92 & 05366271 & Bridge Ck nr Augusta & $44^{\circ} 39^{\prime} 58^{\prime \prime}$ & $91^{\circ} 04^{\prime} 45^{\prime \prime}$ & 05379500 & 13.7 & .0560 & equation & 2.9 & 2.9 \\
\hline 93 & 05366274 & Hay Ck nr Augusta & 4439'33" & $91^{\circ} 04^{\prime} 47^{\prime \prime}$ & 05379500 & 7.85 & .1800 & equation & 3.0 & 5.3 \\
\hline 94 & 05366280 & Bridge $\mathrm{Ck}$ at Augusta & $44^{\circ} 40^{\prime} 41^{\prime \prime}$ & $91^{\circ} 06^{\prime} 15^{\prime \prime}$ & 05379500 & 26.7 & .1854 & equation & 10.8 & 5.5 \\
\hline 95 & 05366285 & Diamomd Valley $\mathrm{Ck}$ at Augusta & $44^{\circ} 40^{\prime} 41^{\prime \prime}$ & $91^{\circ} 06^{\prime} 24^{\prime \prime}$ & 05379500 & 8.26 & .2672 & equation & 3.9 & 6.5 \\
\hline 96 & 05366320 & Bridge $\mathrm{Ck}$ at Augusta & $44^{\circ} 41^{\prime} 04^{\prime \prime}$ & $91^{\circ} 07^{\prime} 33^{\prime \prime}$ & 05379500 & 35.6 & .1640 & relation & 14.0 & 5.3 \\
\hline
\end{tabular}

${ }^{1}$ Low-flow discharge measurements at partial-record stations were compared to one or more nearby USGS gaging stations to establish a relation between measured discharge and concurrent daily discharge. The nearby gaging station that provided the best relation with the partial-record station was chosen as the index station and was associated with nearby miscellaneous measurement sites.

${ }^{2}$ Mismatch between surface-water and ground-water drainage area. 
[USGS, U.S. Geological Survey; ID, identification number; $\mathrm{dd}^{\circ} \mathrm{mm}$ 'ss", degrees, minutes, seconds; $\mathrm{mi}^{2}$, square mile; $\mathrm{ft}^{3} / \mathrm{s} / \mathrm{mi}^{2}$, cubic foot per second per square mile; $\mathrm{ft}^{3} / \mathrm{s}$, cubic foot per second; equation, statewide regression equation; relation, established relation line with index station; R, River; Bk, Brook; Br, Branch; Fk, Fork; Ck, Creek; Tr, Tributary; nr, near; N, North; S, South; E, East; W, West;

--, not determined in historical low-flow reports. See references for full list of low-flow reports.]

\begin{tabular}{|c|c|c|c|c|c|c|c|c|c|c|}
\hline $\begin{array}{c}\text { Map } \\
\text { number }\end{array}$ & $\begin{array}{l}\text { USGS } \\
\text { station ID }\end{array}$ & Station name & $\begin{array}{c}\text { Latitude } \\
\left.\text { (dd }{ }^{\circ} \mathrm{mm} ' s s^{\prime \prime}\right)\end{array}$ & $\begin{array}{l}\text { Longitude } \\
\text { (dd }{ }^{\circ} \mathrm{mm} \text { 'ss") }\end{array}$ & $\begin{array}{l}\text { Index } \\
\text { station }^{1}\end{array}$ & $\begin{array}{c}\text { Drainage } \\
\text { area } \\
\left(\mathrm{mi}^{2}\right)\end{array}$ & $\begin{array}{c}\text { Base flow } \\
\text { factor } \\
\left(\mathrm{ft}^{3} / \mathrm{s} / \mathrm{mi}^{2}\right)\end{array}$ & $\begin{array}{l}\text { Base flow } \\
\text { method }\end{array}$ & $\begin{array}{l}\text { Base flow } \\
\qquad\left(\mathrm{ft}^{3} / \mathrm{s}\right)\end{array}$ & $\begin{array}{c}\text { Recharge } \\
\text { (inches) }\end{array}$ \\
\hline 97 & 05366325 & Thompson Valley Ck nr Augusta & $44^{\circ} 40^{\prime} 35^{\prime \prime}$ & $91^{\circ} 09^{\prime} 52^{\prime \prime}$ & 05366500 & 11.1 & 0.3255 & equation & 5.9 & 7.2 \\
\hline 98 & 05366339 & Browns Ck nr Augusta & $44^{\circ} 43^{\prime} 56^{\prime \prime}$ & $91^{\circ} 08^{\prime} 39^{\prime \prime}$ & 05366500 & 11.8 & .0938 & equation & 3.3 & 3.8 \\
\hline 99 & 05366350 & Bridge Ck nr Augusta & $44^{\circ} 44^{\prime} 21^{\prime \prime}$ & $91^{\circ} 09^{\prime} 55^{\prime \prime}$ & 05366500 & 71.5 & .3318 & equation & 39.8 & 7.6 \\
\hline 100 & 05366400 & Bears Grass Ck nr Fall Ck & $44^{\circ} 43^{\prime} 51^{\prime \prime}$ & $91^{\circ} 11^{\prime} 57^{\prime \prime}$ & 05366500 & 16.2 & .2710 & relation & 3.8 & 3.2 \\
\hline 101 & 05366405 & Bears Grass Ck Tr nr Augusta & $44^{\circ} 43^{\prime} 28^{\prime \prime}$ & $91^{\circ} 11^{\prime} 29^{\prime \prime}$ & 05366500 & 7.84 & .1042 & equation & 2.3 & 4.0 \\
\hline 102 & 05366408 & Bears Grass Ck nr Fall Ck & $44^{\circ} 45^{\prime} 07^{\prime \prime}$ & $91^{\circ} 11^{\prime} 26^{\prime \prime}$ & 05366500 & 28 & .3390 & equation & 15.5 & 7.5 \\
\hline 103 & 05366419 & Rush Ck nr Fall Ck & $44^{\circ} 46^{\prime} 34^{\prime \prime}$ & $91^{\circ} 14^{\prime} 42^{\prime \prime}$ & 05366500 & 6.19 & .0300 & equation & .9 & 2.1 \\
\hline 104 & 05366440 & Fall Ck at Fall Ck & $44^{\circ} 46^{\prime} 05^{\prime \prime}$ & $91^{\circ} 16^{\prime} 21^{\prime \prime}$ & 05379500 & 15.9 & .0860 & relation & 4.0 & 3.4 \\
\hline 105 & 05366600 & Otter Ck at Brackett & $44^{\circ} 42^{\prime} 05^{\prime \prime}$ & $91^{\circ} 20^{\prime} 35^{\prime \prime}$ & 05379500 & 23.7 & .2584 & equation & 11.3 & 6.5 \\
\hline 106 & 05366660 & Beaver Ck at Brackett & $44^{\circ} 41^{\prime} 52^{\prime \prime}$ & $91^{\circ} 21^{\prime} 10^{\prime \prime}$ & 05379500 & 12.4 & .0626 & equation & 2.8 & 3.1 \\
\hline 107 & 05367032 & Lowes Ck nr Eau Claire & $44^{\circ} 44^{\prime} 31^{\prime \prime}$ & $91^{\circ} 27^{\prime} 40^{\prime \prime}$ & 05379500 & 53.8 & .2900 & relation & 34.4 & 8.7 \\
\hline 108 & 05367041 & W Ck nr Eau Claire & $44^{\circ} 45^{\prime} 21^{\prime \prime}$ & $91^{\circ} 37^{\prime} 26^{\prime \prime}$ & 05366500 & 18.4 & .1776 & equation & 7.2 & 5.3 \\
\hline 109 & 05367051 & Elk Ck nr Elk Mound & $44^{\circ} 54^{\prime} 00^{\prime \prime}$ & $91^{\circ} 37^{\prime} 55^{\prime \prime}$ & 05366500 & 49.5 & .4975 & equation & 33.8 & 9.3 \\
\hline 110 & 05367052 & Elk Ck nr Elk Mound & $44^{\circ} 50^{\prime} 26^{\prime \prime}$ & $91^{\circ} 39^{\prime} 36^{\prime \prime}$ & 05366500 & 64 & .3722 & equation & 37.7 & 8.0 \\
\hline 111 & 05367057 & Rock Ck nr Rock Falls & $44^{\circ} 42^{\prime} 15^{\prime \prime}$ & $91^{\circ} 41^{\prime} 20^{\prime \prime}$ & 05379500 & 30.5 & .0956 & equation & 8.7 & 3.9 \\
\hline 112 & 05367060 & Muddy Ck nr Menomonie & $44^{\circ} 48^{\prime} 00^{\prime \prime}$ & $91^{\circ} 46^{\prime} 02^{\prime \prime}$ & 05366500 & 67.4 & .0450 & relation & 7.1 & 1.4 \\
\hline 113 & 05367063 & Cranberry $\mathrm{Ck}$ at Meridean & $44^{\circ} 44^{\prime} 06^{\prime \prime}$ & $91^{\circ} 47^{\prime} 31^{\prime \prime}$ & 05379500 & 16.9 & .2022 & equation & 7.1 & 5.7 \\
\hline 114 & 05367072 & Fall Ck nr Meridean & $44^{\circ} 41^{\prime} 02^{\prime \prime}$ & $91^{\circ} 50^{\prime} 57^{\prime \prime}$ & 05379500 & 8.21 & .3516 & equation & 4.5 & 7.5 \\
\hline 115 & 05367102 & Red Cedar R at Birchwood & $45^{\circ} 39^{\prime} 33^{\prime \prime}$ & $91^{\circ} 33^{\prime} 36^{\prime \prime}$ & 05362000 & 70.8 & .1050 & relation & 37.0 & 7.1 \\
\hline 116 & 05367150 & Sucker Ck nr Birchwood & $45^{\circ} 39^{\prime} 00^{\prime \prime}$ & $91^{\circ} 31^{\prime} 15^{\prime \prime}$ & 05362000 & 8.6 & .0702 & equation & 2.0 & 3.2 \\
\hline 117 & 05367190 & Hemlock Ck nr Mikana & $45^{\circ} 34^{\prime} 27^{\prime \prime}$ & $91^{\circ} 30^{\prime} 46^{\prime \prime}$ & 05368000 & 20.4 & .3114 & equation & 10.7 & 7.1 \\
\hline 118 & 05367202 & $\begin{array}{l}\text { Red Cedar Ck at Red Cedar Lk } \\
\text { Outlet }\end{array}$ & $45^{\circ} 35^{\prime} 21^{\prime \prime}$ & $91^{\circ} 36^{\prime} 06^{\prime \prime}$ & 05368000 & 151 & .3530 & equation & 88.1 & 7.9 \\
\hline 119 & 05367210 & Red Cedar Ck nr Mikana & $45^{\circ} 33^{\prime} 59^{\prime \prime}$ & $91^{\circ} 37^{\prime} 27^{\prime \prime}$ & 05368000 & 159 & .2078 & equation & 70.5 & 6.0 \\
\hline
\end{tabular}

${ }^{1}$ Low-flow discharge measurements at partial-record stations were compared to one or more nearby USGS gaging stations to establish a relation between measured discharge and concurrent daily discharge. The nearby gaging station that provided the best relation with the partial-record station was chosen as the index station and was associated with nearby miscellaneous measurement sites.

${ }^{2}$ Mismatch between surface-water and ground-water drainage area. 
Appendix 3D. Average annual baseflow and corresponding recharge, 1970-99, for partial-record stations in the Chippewa River Basin-Continued.

[USGS, U.S. Geological Survey; ID, identification number; $\mathrm{dd}^{\circ} \mathrm{mm}$ 'ss", degrees, minutes, seconds; $\mathrm{mi}^{2}$, square mile; $\mathrm{ft}^{3} / \mathrm{s} / \mathrm{mi}^{2}$, cubic foot per second per square mile; $\mathrm{ft}^{3} / \mathrm{s}$, cubic foot per second; equation, statewide regression equation; relation, established relation line with index station; R, River; Bk, Brook; Br, Branch; Fk, Fork; Ck, Creek; Tr, Tributary; nr, near; N, North; S, South; E, East; W, West; ,-- not determined in historical low-flow reports. See references for full list of low-flow reports.]

\begin{tabular}{|c|c|c|c|c|c|c|c|c|c|c|}
\hline $\begin{array}{c}\text { Map } \\
\text { number }\end{array}$ & $\begin{array}{c}\text { USGS } \\
\text { station ID }\end{array}$ & Station name & $\begin{array}{c}\text { Latitude } \\
\text { (dd }{ }^{\circ} \mathrm{mm} \text { 'ss") }\end{array}$ & $\begin{array}{c}\text { Longitude } \\
\text { (dd }{ }^{\circ} \mathrm{mm} \text { 'ss") }\end{array}$ & $\begin{array}{l}\text { Index } \\
\text { station }^{1}\end{array}$ & $\begin{array}{c}\text { Drainage } \\
\text { area } \\
\left(\mathrm{mi}^{2}\right)\end{array}$ & $\begin{array}{c}\text { Base flow } \\
\text { factor } \\
\left(\mathrm{ft}^{3} / \mathrm{s} / \mathrm{mi}^{2}\right)\end{array}$ & $\begin{array}{l}\text { Base flow } \\
\text { method }\end{array}$ & $\begin{array}{c}\text { Base flow } \\
\quad\left(\mathrm{ft}^{3} / \mathrm{s}\right)\end{array}$ & $\begin{array}{c}\text { Recharge } \\
\text { (inches) }\end{array}$ \\
\hline 120 & 05367301 & Brill R nr Nobleton & $45^{\circ} 40^{\prime} 03^{\prime \prime}$ & $91^{\circ} 40^{\prime} 48^{\prime \prime}$ & 05368000 & 73.8 & 0.3551 & equation & 42.6 & 7.8 \\
\hline 121 & 05367310 & Brill $\mathrm{R}$ at Brill & $45^{\circ} 36^{\prime} 36^{\prime \prime}$ & $91^{\circ} 40^{\prime} 24^{\prime \prime}$ & 05368000 & 84.6 & .3590 & relation & 52.0 & 8.3 \\
\hline 122 & 05367340 & Red Cedar R nr Campia & $45^{\circ} 33^{\prime} 08^{\prime \prime}$ & $91^{\circ} 39^{\prime} 15^{\prime \prime}$ & 05368000 & 258 & .2444 & equation & 125.7 & 6.6 \\
\hline 123 & 05367350 & Red Cedar R nr Rice Lk & $45^{\circ} 31^{\prime} 49^{\prime \prime}$ & $91^{\circ} 42^{\prime} 10^{\prime \prime}$ & 05368000 & 264 & .4474 & equation & 176.2 & 9.1 \\
\hline 124 & 05367401 & Bear Ck at Haugen & $45^{\circ} 36^{\prime} 35^{\prime \prime}$ & $91^{\circ} 464^{\prime \prime}$ & 05368000 & 47.6 & -- & relation & 14.7 & 4.2 \\
\hline 125 & 05367402 & Little Bear Ck nr Haugen & $45^{\circ} 34^{\prime} 21^{\prime \prime}$ & $91^{\circ} 47^{\prime} 09^{\prime \prime}$ & 05368000 & 17.2 & .2353 & equation & 7.8 & 6.1 \\
\hline 126 & 05367404 & Bear Ck nr Rice Lk & $45^{\circ} 32^{\prime} 12^{\prime \prime}$ & $91^{\circ} 44^{\prime} 52^{\prime \prime}$ & 05368000 & 77.8 & .2345 & equation & 36.2 & 6.3 \\
\hline 127 & 05367405 & Red Cedar R at Rice Lk & $45^{\circ} 29^{\prime} 55^{\prime \prime}$ & $91^{\circ} 44^{\prime} 12^{\prime \prime}$ & 05368000 & 372 & .4164 & equation & 240.8 & 8.8 \\
\hline 128 & 05367409 & Spring Ck nr Campia & $45^{\circ} 31^{\prime} 57^{\prime \prime}$ & $91^{\circ} 34^{\prime} 48^{\prime \prime}$ & 05368000 & 11.3 & .0078 & equation & .9 & 1.0 \\
\hline 129 & 05367412 & Spring Ck nr Rice Lk & $45^{\circ} 29^{\prime} 14^{\prime \prime}$ & $91^{\circ} 41^{\prime} 15^{\prime \prime}$ & 05368000 & 30.8 & .1118 & equation & 9.6 & 4.2 \\
\hline 130 & 05367417 & Red Cedar? nr Rice Lk & $45^{\circ} 26^{\prime} 42^{\prime \prime}$ & $91^{\circ} 45^{\prime} 54^{\prime \prime}$ & 05368000 & 421 & .4306 & equation & 278.0 & 9.0 \\
\hline 131 & 05367420 & Barker Ck nr Barron & $45^{\circ} 24^{\prime} 31^{\prime \prime}$ & $91^{\circ} 47^{\prime} 11^{\prime \prime}$ & 05368000 & 16.5 & .1850 & relation & 5.7 & 4.7 \\
\hline 132 & 05367425 & Red Cedar R nr Cameron & $45^{\circ} 24^{\prime} 05^{\prime \prime}$ & $91^{\circ} 46^{\prime} 39^{\prime \prime}$ & 05368000 & 442 & .3953 & equation & 279.5 & 8.6 \\
\hline 133 & 05367427 & Cranberry $\mathrm{Ck}$ at Cameron & $45^{\circ} 24^{\prime} 30^{\prime \prime}$ & $91^{\circ} 45^{\prime} 05^{\prime \prime}$ & 05368000 & 3.45 & .8150 & relation & 4.2 & ${ }^{2} 16.5$ \\
\hline 134 & 05367430 & Yellow R nr Cumberland & $45^{\circ} 31^{\prime} 54^{\prime \prime}$ & $91^{\circ} 54^{\prime} 56^{\prime \prime}$ & 05368000 & 50.4 & .2360 & relation & 16.4 & 4.4 \\
\hline 135 & 05367433 & Hickey Ck nr Haugen & $45^{\circ} 31^{\prime} 22^{\prime \prime}$ & $91^{\circ} 52^{\prime} 33^{\prime \prime}$ & 05368000 & 8.59 & .4261 & equation & 5.2 & 8.3 \\
\hline 136 & 05367434 & Eagle Ck nr Rice Lk & $45^{\circ} 30^{\prime} 09^{\prime \prime}$ & $91^{\circ} 51^{\prime} 43^{\prime \prime}$ & 05368000 & 7.04 & .0976 & equation & 2.0 & 3.8 \\
\hline 137 & 05367436 & Yellow R nr Barron & $45^{\circ} 26^{\prime} 15^{\prime \prime}$ & $91^{\circ} 53^{\prime} 00^{\prime \prime}$ & 05368000 & 85.5 & .4487 & equation & 55.9 & 8.9 \\
\hline 138 & 05367440 & Vermillion R nr Almena & $45^{\circ} 26^{\prime} 12^{\prime \prime}$ & $91^{\circ} 57^{\prime} 56^{\prime \prime}$ & 05341500 & 18.2 & .3270 & relation & 9.3 & 6.9 \\
\hline 139 & 05367443 & Vermillion R nr Poskin & $45^{\circ} 24^{\prime} 36^{\prime \prime}$ & $91^{\circ} 53^{\prime} 20^{\prime \prime}$ & 05368000 & 40.2 & .2635 & equation & 19.6 & 6.6 \\
\hline 140 & 05367444 & Yellow R at Barron & $45^{\circ} 23^{\prime} 57^{\prime \prime}$ & $91^{\circ} 50^{\prime} 07^{\prime \prime}$ & 05368000 & 141 & .3640 & relation & 97.2 & 9.4 \\
\hline 141 & 05367445 & Quaderer Ck at Barron & $45^{\circ} 23^{\prime} 57^{\prime \prime}$ & $91^{\circ} 50^{\prime} 33^{\prime \prime}$ & 05368000 & 11.5 & .2455 & equation & 5.3 & 6.2 \\
\hline 142 & 05367446 & Yellow R at Barron & $45^{\circ} 23^{\prime} 53^{\prime \prime}$ & $91^{\circ} 49^{\prime} 59^{\prime \prime}$ & 05368000 & 153 & .2405 & equation & 73.1 & 6.5 \\
\hline 143 & 05367447 & Fourmile Ck nr Barron & $45^{\circ} 22^{\prime} 58^{\prime \prime}$ & $91^{\circ} 49^{\prime} 39^{\prime \prime}$ & 05368000 & 15.6 & .2386 & equation & 7.1 & 6.2 \\
\hline
\end{tabular}

${ }^{1}$ Low-flow discharge measurements at partial-record stations were compared to one or more nearby USGS gaging stations to establish a relation between measured discharge and concurrent daily discharge. The nearby gaging station that provided the best relation with the partial-record station was chosen as the index station and was associated with nearby miscellaneous measurement sites.

${ }^{2}$ Mismatch between surface-water and ground-water drainage area. 
[USGS, U.S. Geological Survey; ID, identification number; $\mathrm{dd}^{\circ} \mathrm{mm}$ 'ss", degrees, minutes, seconds; $\mathrm{mi}^{2}$, square mile; $\mathrm{ft}^{3} / \mathrm{s} / \mathrm{mi}^{2}$, cubic foot per second per square mile; $\mathrm{ft}^{3} / \mathrm{s}$, cubic foot per second; equation, statewide regression equation; relation, established relation line with index station; R, River; Bk, Brook; Br, Branch; Fk, Fork; Ck, Creek; Tr, Tributary; nr, near; N, North; S, South; E, East; W, West;

--, not determined in historical low-flow reports. See references for full list of low-flow reports.]

\begin{tabular}{|c|c|c|c|c|c|c|c|c|c|c|}
\hline $\begin{array}{c}\text { Map } \\
\text { number }\end{array}$ & $\begin{array}{c}\text { USGS } \\
\text { station ID }\end{array}$ & Station name & $\begin{array}{c}\text { Latitude } \\
\text { (dd }{ }^{\circ} \mathrm{mm} \text { 'ss") }\end{array}$ & $\begin{array}{c}\text { Longitude } \\
\text { (dd }{ }^{\circ} \mathrm{mm} \text { 'ss") }\end{array}$ & $\begin{array}{l}\text { Index } \\
\text { station }{ }^{1}\end{array}$ & $\begin{array}{c}\text { Drainage } \\
\text { area } \\
\left(\mathrm{mi}^{2}\right)\end{array}$ & $\begin{array}{c}\text { Base flow } \\
\text { factor } \\
\left(\mathrm{ft}^{3} / \mathrm{s} / \mathrm{mi}^{2}\right)\end{array}$ & $\begin{array}{l}\text { Base flow } \\
\text { method }\end{array}$ & $\begin{array}{c}\text { Base flow } \\
\left(\mathrm{ft}^{3} / \mathrm{s}\right)\end{array}$ & $\begin{array}{c}\text { Recharge } \\
\text { (inches) }\end{array}$ \\
\hline 144 & 05367448 & Yellow R nr Wickware & $45^{\circ} 21^{\prime} 56^{\prime \prime}$ & $91^{\circ} 47^{\prime} 12^{\prime \prime}$ & 05368000 & 176 & 0.3539 & equation & 103.1 & 8.0 \\
\hline 145 & 05367450 & Red Cedar R nr Chetek & $45^{\circ} 21^{\prime} 03^{\prime \prime}$ & $91^{\circ} 44^{\prime} 47^{\prime \prime}$ & 05368000 & 631 & .3861 & equation & 396.9 & 8.5 \\
\hline 146 & 05367452 & Brown Ck nr Chetek & $45^{\circ} 20^{\prime} 40^{\prime \prime}$ & $91^{\circ} 44^{\prime} 25^{\prime \prime}$ & 05368000 & 9.91 & .1763 & equation & 3.8 & 5.2 \\
\hline 147 & 05367454 & Red Cedar R nr Chetek & $45^{\circ} 19^{\prime} 17^{\prime \prime}$ & $91^{\circ} 41^{\prime} 28^{\prime \prime}$ & 05368000 & 648 & .3653 & equation & 396.3 & 8.3 \\
\hline 148 & 05367457 & Silver Ck nr Rice Lk & $45^{\circ} 28^{\prime} 22^{\prime \prime}$ & $91^{\circ} 38^{\prime} 03^{\prime \prime}$ & 05368000 & 3.57 & .0532 & equation & .7 & 2.7 \\
\hline 149 & 05367459 & Rock Ck nr Canton & $45^{\circ} 27^{\prime} 19^{\prime \prime}$ & $91^{\circ} 38^{\prime} 37^{\prime \prime}$ & 05368000 & 18.9 & .0542 & equation & 4.0 & 2.9 \\
\hline 150 & 05367460 & Pokegama Ck nr Cameron & $45^{\circ} 24^{\prime} 29^{\prime \prime}$ & $91^{\circ} 39^{\prime} 36^{\prime \prime}$ & 05368000 & 39.7 & .3200 & relation & 23.4 & 8.0 \\
\hline 151 & 05367461 & German Ck nr Cameron & $45^{\circ} 24^{\prime} 28^{\prime \prime}$ & $91^{\circ} 39^{\prime} 10^{\prime \prime}$ & 05368000 & 8.16 & .0490 & equation & 1.6 & 2.7 \\
\hline 152 & 05367462 & Moose Ear Ck nr Strickland & $45^{\circ} 24^{\prime} 28^{\prime \prime}$ & $91^{\circ} 33^{\prime} 16^{\prime \prime}$ & 05368000 & 13.2 & .1107 & equation & 4.0 & 4.1 \\
\hline 153 & 05367463 & Moose Ear Ck nr Chetek & $45^{\circ} 19^{\prime} 15^{\prime \prime}$ & $91^{\circ} 35^{\prime} 17^{\prime \prime}$ & 05368000 & 38.2 & .2390 & equation & 17.7 & 6.3 \\
\hline 154 & 053674633 & Chetek $\mathrm{R}$ at Chetek & $45^{\circ} 18^{\prime} 32^{\prime \prime}$ & $91^{\circ} 39^{\prime} 04^{\prime \prime}$ & 05368000 & 135 & -- & relation & 125.0 & 12.6 \\
\hline 155 & 05367464 & Tenmile Ck nr Chetek & $45^{\circ} 17^{\prime} 09^{\prime \prime}$ & $91^{\circ} 33^{\prime} 40^{\prime \prime}$ & 05368000 & 30.3 & .4512 & equation & 19.5 & 8.7 \\
\hline 156 & 05367468 & Chetek R nr Chetek & $45^{\circ} 16^{\prime} 22^{\prime \prime}$ & $91^{\circ} 41^{\prime} 41^{\prime \prime}$ & 05368000 & 212 & .6017 & equation & 164.0 & 10.5 \\
\hline 157 & 05367469 & Red Cedar R nr Dallas & $45^{\circ} 14^{\prime} 52^{\prime \prime}$ & $91^{\circ} 43^{\prime} 26^{\prime \prime}$ & 05368000 & 887 & .3996 & equation & 571.9 & 8.8 \\
\hline 158 & 05367474 & Upper Pine Ck nr Dallas & $45^{\circ} 16^{\prime} 24^{\prime \prime}$ & $91^{\circ} 49^{\prime} 31^{\prime \prime}$ & 05368000 & 8.79 & -- & relation & 6.3 & 9.7 \\
\hline 159 & 05367480 & E Br Upper Pine Ck Tr nr Dallas & $45^{\circ} 16^{\prime} 52^{\prime \prime}$ & $91^{\circ} 48^{\prime} 30^{\prime \prime}$ & 05368000 & 3.95 & .3130 & relation & 1.5 & 5.2 \\
\hline 160 & 05367481 & E Br Upper Pine Ck at Dallas & $45^{\circ} 16^{\prime} 03^{\prime \prime}$ & $91^{\circ} 49^{\prime} 04^{\prime \prime}$ & 05368000 & 9.66 & .6749 & equation & 7.5 & 10.5 \\
\hline 161 & 053674825 & Upper Pine Ck nr Dallas & $45^{\circ} 12^{\prime} 57^{\prime \prime}$ & $91^{\circ} 45^{\prime} 46^{\prime \prime}$ & 05368000 & 36.3 & .4360 & equation & 23.0 & 8.6 \\
\hline 162 & 05367487 & Sand Ck at Sand Ck & $45^{\circ} 10^{\prime} 00^{\prime \prime}$ & $91^{\circ} 41^{\prime} 08^{\prime \prime}$ & 05368000 & 20 & .4899 & equation & 13.3 & 9.0 \\
\hline 163 & 053674879 & Lower Pine Ck nr Ridgeland & $45^{\circ} 13^{\prime} 49^{\prime \prime}$ & $91^{\circ} 52^{\prime} 49^{\prime \prime}$ & 05368000 & 19.7 & .0602 & equation & 4.4 & 3.0 \\
\hline 164 & 05367488 & S Fk Lower Pine Ck at Ridgeland & $45^{\circ} 12^{\prime} 26^{\prime \prime}$ & $91^{\circ} 53^{\prime} 37^{\prime \prime}$ & 05368000 & 7.93 & -- & relation & 1.5 & 2.6 \\
\hline 165 & 05367490 & Lower Pine Ck at Sand Ck & $45^{\circ} 10^{\prime} 04^{\prime \prime}$ & $91^{\circ} 42^{\prime} 03^{\prime \prime}$ & 05368000 & 51 & .3130 & relation & 40.9 & 10.9 \\
\hline 166 & 05367491 & Red Cedar R nr Sand Ck & $45^{\circ} 08^{\prime} 34^{\prime \prime}$ & $91^{\circ} 42^{\prime} 15^{\prime \prime}$ & 05368000 & 1020 & .4344 & equation & 688.8 & 9.2 \\
\hline 167 & 05367492 & Hay Ck nr Sand Ck & $45^{\circ} 09^{\prime} 05^{\prime \prime}$ & $91^{\circ} 43^{\prime} 40^{\prime \prime}$ & 05368000 & 15.2 & .2349 & equation & 6.9 & 6.1 \\
\hline
\end{tabular}

${ }^{1}$ Low-flow discharge measurements at partial-record stations were compared to one or more nearby USGS gaging stations to establish a relation between measured discharge and concurrent daily discharge. The nearby gaging station that provided the best relation with the partial-record station was chosen as the index station and was associated with nearby miscellaneous measurement sites.

${ }^{2}$ Mismatch between surface-water and ground-water drainage area. 
Appendix 3D. Average annual baseflow and corresponding recharge, 1970-99, for partial-record stations in the Chippewa River Basin-Continued.

[USGS, U.S. Geological Survey; ID, identification number; $\mathrm{dd}^{\circ} \mathrm{mm}$ 'ss", degrees, minutes, seconds; $\mathrm{mi}^{2}$, square mile; $\mathrm{ft}^{3} / \mathrm{s} / \mathrm{mi}^{2}$, cubic foot per second per square mile; $\mathrm{ft}^{3} / \mathrm{s}$, cubic foot per second; equation, statewide regression equation; relation, established relation line with index station; R, River; Bk, Brook; Br, Branch; Fk, Fork; Ck, Creek; Tr, Tributary; nr, near; N, North; S, South; E, East; W, West;

--, not determined in historical low-flow reports. See references for full list of low-flow reports.]

\begin{tabular}{|c|c|c|c|c|c|c|c|c|c|c|}
\hline $\begin{array}{c}\text { Map } \\
\text { number }\end{array}$ & $\begin{array}{c}\text { USGS } \\
\text { station ID }\end{array}$ & Station name & $\begin{array}{c}\text { Latitude } \\
\text { (dd }{ }^{\circ} \mathrm{mm} \text { 'ss") }\end{array}$ & $\begin{array}{c}\text { Longitude } \\
\text { (ddºmm'ss") }\end{array}$ & $\begin{array}{l}\text { Index } \\
\text { station }{ }^{1}\end{array}$ & $\begin{array}{c}\text { Drainage } \\
\text { area } \\
\left(\mathrm{mi}^{2}\right)\end{array}$ & $\begin{array}{c}\text { Base flow } \\
\text { factor } \\
\left(\mathrm{ft}^{3} / \mathrm{s} / \mathrm{mi}^{2}\right)\end{array}$ & $\begin{array}{c}\text { Base flow } \\
\text { method }\end{array}$ & $\begin{array}{c}\text { Base flow } \\
\quad\left(\mathrm{ft}^{3} / \mathrm{s}\right)\end{array}$ & $\begin{array}{c}\text { Recharge } \\
\text { (inches) }\end{array}$ \\
\hline 168 & 05367495 & Popple Ck nr Sand Ck & $45^{\circ} 06^{\prime} 12^{\prime \prime}$ & $91^{\circ} 43^{\prime} 25^{\prime \prime}$ & 05368000 & 8.04 & 0.3123 & equation & 4.2 & 7.0 \\
\hline 169 & 05367497 & Trout Ck nr Sand Ck & $46^{\circ} 06^{\prime} 19^{\prime \prime}$ & $91^{\circ} 41^{\prime} 31^{\prime \prime}$ & 05333500 & 30 & .3202 & equation & 16.1 & 7.3 \\
\hline 170 & 05367515 & Eighteenmile Ck nr Colfax & $45^{\circ} 00^{\prime} 06^{\prime \prime}$ & $91^{\circ} 41^{\prime} 29^{\prime \prime}$ & 05368000 & 21.5 & .4177 & equation & 13.2 & 8.3 \\
\hline 171 & 05367630 & Hay $\mathrm{R}$ at Cumberland & $45^{\circ} 31^{\prime} 29^{\prime \prime}$ & $92^{\circ} 00^{\prime} 58^{\prime \prime}$ & 05368000 & 12 & .6948 & equation & 9.5 & 10.7 \\
\hline 172 & 05367642 & Hay R nr Cumberland & $45^{\circ} 27^{\prime} 03^{\prime \prime}$ & $92^{\circ} 00^{\prime} 05^{\prime \prime}$ & 05368000 & 27.9 & .3318 & equation & 15.2 & 7.4 \\
\hline 173 & 05367700 & Lightning $\mathrm{Ck}$ at Almena & $45^{\circ} 25^{\prime} 17^{\prime \prime}$ & $92^{\circ} 01 ' 57^{\prime \prime}$ & 05368000 & 19 & -- & relation & 3.2 & 2.3 \\
\hline 174 & 05367750 & Dority Ck nr Arland & $45^{\circ} 17^{\prime} 32^{\prime \prime}$ & $91^{\circ} 57^{\prime} 45^{\prime \prime}$ & 05368000 & 7.62 & .4931 & equation & 5.0 & 8.9 \\
\hline 175 & 05367770 & Hay R nr Prairie Farm & $45^{\circ} 15^{\prime} 45^{\prime \prime}$ & $91^{\circ} 58^{\prime} 49^{\prime \prime}$ & 05368000 & 92.1 & -- & relation & 46.6 & 6.9 \\
\hline 176 & 05367772 & Turtle Ck nr Turtle Lk & $45^{\circ} 24^{\prime} 27^{\prime \prime}$ & $92^{\circ} 05^{\prime} 15^{\prime \prime}$ & 05368000 & 3.76 & .5350 & equation & 2.5 & 9.0 \\
\hline 177 & 05367800 & Turtle Ck nr Almena & $45^{\circ} 18^{\prime} 33^{\prime \prime}$ & $92^{\circ} 03^{\prime} 02^{\prime \prime}$ & 05368000 & 39.4 & .2740 & relation & 24.8 & 8.5 \\
\hline 178 & 05367807 & Turtle Ck nr Arland & $45^{\circ} 15^{\prime} 20^{\prime \prime}$ & $92^{\circ} 00^{\prime} 13^{\prime \prime}$ & 05368000 & 60.8 & .2856 & equation & 31.2 & 7.0 \\
\hline 179 & 05367834 & Hay R nr Ridgeland & $45^{\circ} 08^{\prime} 10^{\prime \prime}$ & $91^{\circ} 56^{\prime} 14^{\prime \prime}$ & 05368000 & 200 & .4154 & equation & 127.7 & 8.7 \\
\hline 180 & 05367860 & Big Beaver Ck nr Wheeler & $45^{\circ} 06^{\prime} 09^{\prime \prime}$ & $91^{\circ} 56^{\prime} 44^{\prime \prime}$ & 05368000 & 18.9 & .0810 & equation & 4.9 & 3.5 \\
\hline 181 & 05367880 & S Fk Hay R at Reeve & $45^{\circ} 14^{\prime} 19^{\prime \prime}$ & $92^{\circ} 08^{\prime} 10^{\prime \prime}$ & 05368000 & 15.8 & .0095 & equation & 1.3 & 1.2 \\
\hline 182 & 05367930 & S Fk Hay R nr Boyceville & $45^{\circ} 04^{\prime} 17^{\prime \prime}$ & $92^{\circ} 02^{\prime} 08^{\prime \prime}$ & 05368000 & 89.8 & .3710 & relation & 45.0 & 6.8 \\
\hline 183 & 05367942 & Tiffany Ck at Glenwood City & $45^{\circ} 02^{\prime} 47^{\prime \prime}$ & $92^{\circ} 09^{\prime} 24^{\prime \prime}$ & 05368000 & 13.7 & .3310 & relation & 6.6 & 6.5 \\
\hline 184 & 05367970 & Beaver Ck at Downing & $45^{\circ} 02^{\prime} 46^{\prime \prime}$ & $92^{\circ} 07^{\prime} 32^{\prime \prime}$ & 05368000 & 17.6 & .2440 & relation & 6.3 & 4.9 \\
\hline 185 & 05367975 & Tiffany $\mathrm{Ck}$ at Boyceville & $45^{\circ} 02^{\prime} 48^{\prime \prime}$ & $92^{\circ} 02^{\prime} 08^{\prime \prime}$ & 05368000 & 69.9 & .2910 & relation & 28.9 & 5.6 \\
\hline 186 & 05367995 & S Fk Hay R nr Wheeler & $45^{\circ} 03^{\prime} 19^{\prime \prime}$ & $91^{\circ} 57^{\prime} 21^{\prime \prime}$ & 05368000 & 180 & .4331 & equation & 117.2 & 8.8 \\
\hline 187 & 05368100 & Otter Ck nr Wheeler & $45^{\circ} 03^{\prime} 36^{\prime \prime}$ & $91^{\circ} 52^{\prime} 43^{\prime \prime}$ & 05368000 & 25.8 & .2660 & relation & 16.7 & 8.8 \\
\hline 188 & 05368120 & Hay R nr Wheeler & $45^{\circ} 02^{\prime} 19^{\prime \prime}$ & $91^{\circ} 52^{\prime} 38^{\prime \prime}$ & 05368000 & 466 & .4015 & equation & 297.3 & 8.7 \\
\hline 189 & 05368168 & Lambs Ck nr Menomonie & $44^{\circ} 59^{\prime} 02^{\prime \prime}$ & $91^{\circ} 53 ' 39^{\prime \prime}$ & 05368000 & 10.5 & .0325 & equation & 1.7 & 2.2 \\
\hline 190 & 05368740 & Wilson Ck nr Knapp & $44^{\circ} 56^{\prime} 37^{\prime \prime}$ & $92^{\circ} 02^{\prime} 10^{\prime \prime}$ & 05368000 & 28 & .3880 & relation & 18.5 & 9.0 \\
\hline 191 & 05368785 & Wilson Ck nr Menomonie & $44^{\circ} 55^{\prime} 05^{\prime \prime}$ & $91^{\circ} 57^{\prime} 54^{\prime \prime}$ & 05368000 & 65.3 & .4979 & equation & 44.8 & 9.3 \\
\hline
\end{tabular}

${ }^{1}$ Low-flow discharge measurements at partial-record stations were compared to one or more nearby USGS gaging stations to establish a relation between measured discharge and concurrent daily discharge. The nearby gaging station that provided the best relation with the partial-record station was chosen as the index station and was associated with nearby miscellaneous measurement sites.

${ }^{2}$ Mismatch between surface-water and ground-water drainage area. 
Appendix 3D. Average annual base flow and corresponding recharge, 1970-99, for partial-record stations in the Chippewa River Basin-Continued.

[USGS, U.S. Geological Survey; ID, identification number; $\mathrm{dd}^{\circ} \mathrm{mm}$ 'ss", degrees, minutes, seconds; $\mathrm{mi}^{2}$, square mile; $\mathrm{ft}^{3} / \mathrm{s} / \mathrm{mi}^{2}$, cubic foot per second per square mile; $\mathrm{ft}^{3} / \mathrm{s}$, cubic foot per second; equation, statewide regression equation; relation, established relation line with index station; R, River; Bk, Brook; Br, Branch; Fk, Fork; Ck, Creek; Tr, Tributary; nr, near; N, North; S, South; E, East; W, West; ,-- not determined in historical low-flow reports. See references for full list of low-flow reports.]

\begin{tabular}{|c|c|c|c|c|c|c|c|c|c|c|}
\hline $\begin{array}{c}\text { Map } \\
\text { number }\end{array}$ & $\begin{array}{c}\text { USGS } \\
\text { station ID }\end{array}$ & Station name & $\begin{array}{c}\text { Latitude } \\
\text { (dd }{ }^{\circ} \mathrm{mm} \text { 'ss") }\end{array}$ & $\begin{array}{c}\text { Longitude } \\
\text { (dd }{ }^{\circ} \mathrm{mm} \text { 'ss") }\end{array}$ & $\begin{array}{l}\text { Index } \\
\text { station }^{1}\end{array}$ & $\begin{array}{c}\text { Drainage } \\
\text { area } \\
\left(\mathrm{mi}^{2}\right)\end{array}$ & $\begin{array}{c}\text { Base flow } \\
\text { factor } \\
\left(\mathrm{ft}^{3} / \mathrm{s} / \mathrm{mi}^{2}\right)\end{array}$ & $\begin{array}{c}\text { Base flow } \\
\text { method }\end{array}$ & $\begin{array}{c}\text { Base flow } \\
\qquad\left(\mathrm{ft}^{3} / \mathrm{s}\right)\end{array}$ & $\begin{array}{r}\text { Recharge } \\
\text { (inches) }\end{array}$ \\
\hline 192 & 05369030 & Gilbert Ck at Menomonie & $44^{\circ} 52^{\prime} 27^{\prime \prime}$ & $91^{\circ} 56^{\prime} 30^{\prime \prime}$ & 05368000 & 36.4 & 0.3530 & relation & 18.9 & 7.1 \\
\hline 193 & 05369050 & Irving Ck nr Irvington & $44^{\circ} 50^{\prime} 35^{\prime \prime}$ & $91^{\circ} 59^{\prime} 00^{\prime \prime}$ & 05379500 & 8.54 & .2777 & equation & 4.2 & 6.6 \\
\hline 194 & 05369110 & Little Elk Ck nr Downsville & $44^{\circ} 48^{\prime} 08^{\prime \prime}$ & $91^{\circ} 54^{\prime} 35^{\prime \prime}$ & 05379500 & 15.4 & .2416 & equation & 7.0 & 6.2 \\
\hline 195 & 05369380 & Bear Ck nr Durand & $44^{\circ} 37^{\prime} 04^{\prime \prime}$ & $91^{\circ} 53^{\prime} 25^{\prime \prime}$ & 05379500 & 37.9 & .2029 & equation & 16.1 & 5.8 \\
\hline 196 & 05369400 & Bear Ck nr Durand & $44^{\circ} 38^{\prime} 42^{\prime \prime}$ & $91^{\circ} 56^{\prime} 09^{\prime \prime}$ & 05379500 & 48.1 & .2530 & relation & 26.0 & 7.3 \\
\hline 197 & 05369850 & Carr Ck at Woodville & $44^{\circ} 56^{\prime} 38^{\prime \prime}$ & $92^{\circ} 17 ' 24 "$ & 05368000 & 11.8 & .0006 & equation & .2 & .3 \\
\hline 198 & 05369900 & Eau Galle R nr Woodville & $44^{\circ} 54^{\prime} 18^{\prime \prime}$ & $92^{\circ} 15^{\prime} 51^{\prime \prime}$ & 05368000 & 39.4 & .1212 & equation & 12.8 & 4.4 \\
\hline 199 & 05370510 & Cady Ck nr Elmwood & $44^{\circ} 47^{\prime} 26^{\prime \prime}$ & $92^{\circ} 06^{\prime} 25^{\prime \prime}$ & 05379500 & 22.4 & 1.0493 & equation & 22.2 & 13.4 \\
\hline 200 & 05370525 & Knights Ck nr Won & $44^{\circ} 47^{\prime} 34^{\prime \prime}$ & $92^{\circ} 01^{\prime} 46^{\prime \prime}$ & 05379500 & 19.1 & .1952 & equation & 7.9 & 5.6 \\
\hline 201 & 05370550 & Eau Galle R at Eau Galle & $44^{\circ} 41^{\prime} 31^{\prime \prime}$ & $92^{\circ} 00^{\prime} 39^{\prime \prime}$ & 05379500 & 181 & .3258 & equation & 101.7 & 7.6 \\
\hline 202 & 05370557 & Missouri $\mathrm{Ck}$ at Eau Galle & $44^{\circ} 41^{\prime} 55^{\prime \prime}$ & $92^{\circ} 01^{\prime} 57^{\prime \prime}$ & 05379500 & 28.2 & .1430 & equation & 9.9 & 4.8 \\
\hline 203 & 05370900 & Spring Ck nr Durand & $44^{\circ} 34^{\prime} 13^{\prime \prime}$ & $91^{\circ} 57^{\prime} 48^{\prime \prime}$ & 05379500 & 6.45 & .1480 & relation & 1.6 & 3.4 \\
\hline 204 & 05371000 & Plum Ck at Plum City & $44^{\circ} 38^{\prime} 13^{\prime \prime}$ & $92^{\circ} 11^{\prime} 10^{\prime \prime}$ & 05379500 & 31.6 & .1130 & relation & 7.7 & 3.3 \\
\hline 205 & 05371035 & Porcupine $\mathrm{Ck}$ at Porcupine & $44^{\circ} 35^{\prime} 03^{\prime \prime}$ & $92^{\circ} 05^{\prime} 42^{\prime \prime}$ & 05379500 & 7.24 & .1633 & equation & 2.7 & 5.0 \\
\hline 206 & 05371270 & Little Bear Ck nr Misha Mokwa & $44^{\circ} 29^{\prime} 55^{\prime \prime}$ & $92^{\circ} 00^{\prime} 35^{\prime \prime}$ & 05379500 & 48.4 & .2555 & equation & 23.3 & 6.5 \\
\hline
\end{tabular}

${ }^{1}$ Low-flow discharge measurements at partial-record stations were compared to one or more nearby USGS gaging stations to establish a relation between measured discharge and concurrent daily discharge. The nearby gaging station that provided the best relation with the partial-record station was chosen as the index station and was associated with nearby miscellaneous measurement sites.

${ }^{2}$ Mismatch between surface-water and ground-water drainage area. 

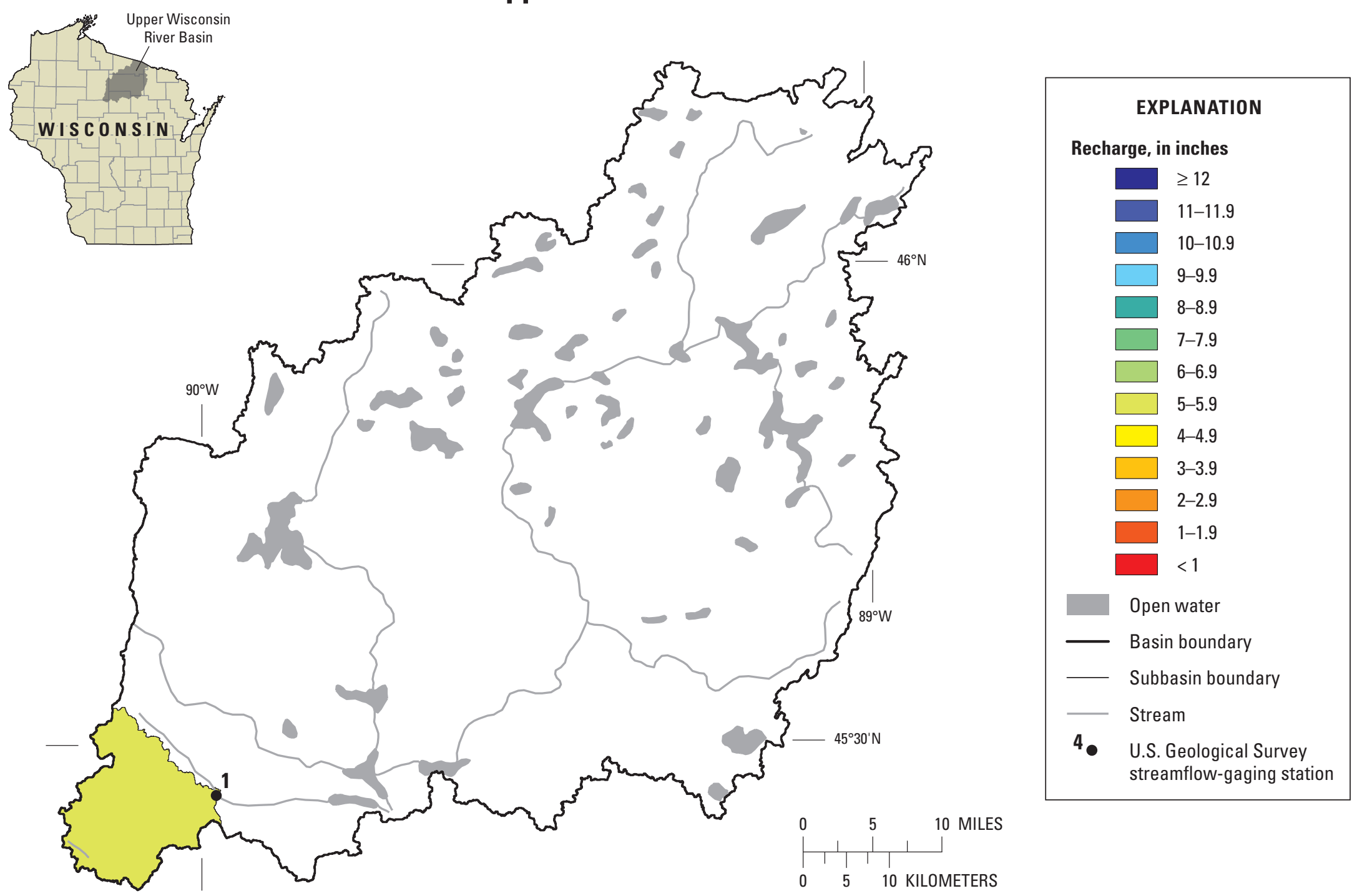

Appendix 4-A. Locations of streamflow-gaging stations in the Upper Wisconsin River Basin and spatial distribution of average annual recharge. 

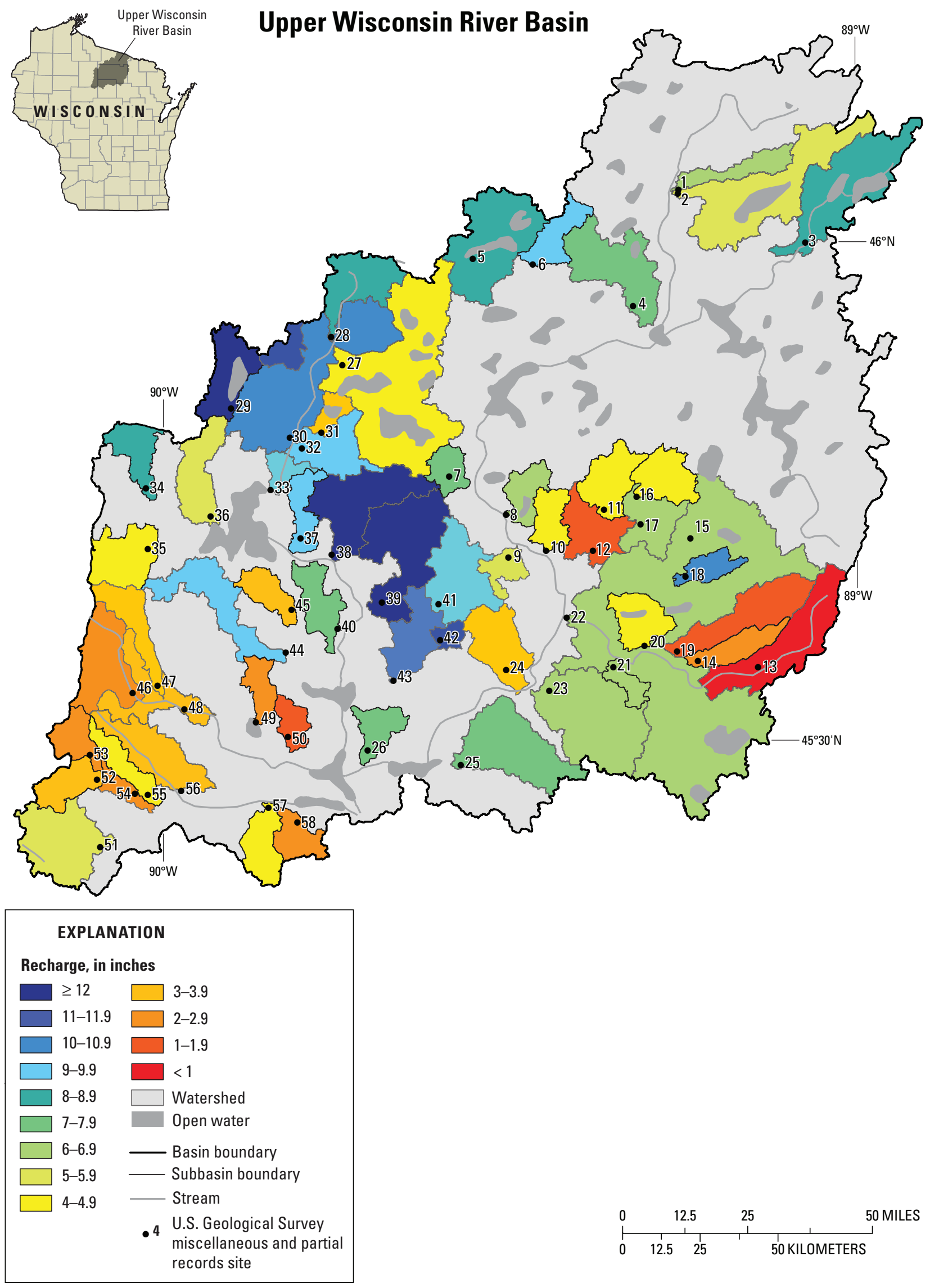

Appendix 4-B. Locations of miscellaneous and partial-record sites in the Upper Wisconsin River Basin and spatial distribution of estimated average annual recharge. 
Appendix 4C. Average annual base flow and corresponding recharge, 1970-99, for streamflow-streamflow-gaging stations in the Upper Wisconsin River Basin.

[USGS, U.S. Geological Survey; ID, identification number; mi² $^{2}$ square mile; fts/s, cubic foot per second; BFI, Baseflow Index; ex rec, extended record; R, River; Bk, Brook; Br, Branch; Fk, Fork; Ck, Creek; Tr, Tributary; nr, near; N, North; S, South; E, East; W, West; --, not determined in historical low-flow reports. See references for full list of low-flow reports. ]

\begin{tabular}{|c|c|c|c|c|c|c|c|c|c|c|c|}
\hline $\begin{array}{c}\text { Map } \\
\text { number }\end{array}$ & $\begin{array}{c}\text { USGS } \\
\text { station } \\
\text { ID }\end{array}$ & Station name & $\begin{array}{c}\text { Drainage } \\
\text { area } \\
\left(\mathrm{mi}^{2}\right)\end{array}$ & $\begin{array}{c}\text { Period of } \\
\text { analysis } \\
\text { (water years }{ }^{1} \text { ) }\end{array}$ & $\begin{array}{c}\text { Base flow } \\
\text { method }\end{array}$ & $\begin{array}{l}\text { Base flow } \\
\text { index }\end{array}$ & $\begin{array}{c}\text { Base flow } \\
\left(\mathrm{ft}^{3} / \mathrm{s}\right)\end{array}$ & $\begin{array}{c}\text { Recharge } \\
\text { (inches) }\end{array}$ & $\begin{array}{c}\text { Total } \\
\text { streamflow } \\
\left(\mathrm{ft}^{3} / \mathrm{s}\right)\end{array}$ & $\begin{array}{c}\text { Total } \\
\text { streamflow } \\
\text { (inches) }\end{array}$ & $\begin{array}{c}{ }^{2} 090 \\
\left(\mathrm{ft}^{3} / \mathrm{s}\right)\end{array}$ \\
\hline 1 & 05393500 & Spirit $\mathrm{R}$ at Spirit Falls & 81.6 & 1970-1999 & BFI & 0.3440 & 32 & 5.3 & 93 & 15.5 & 10 \\
\hline
\end{tabular}
ber 30, 2004). 
Appendix 4D. Average annual base flow and corresponding recharge, 1970-99, for partial-record stations in the Upper Wisconsin River Basin.

[USGS, U.S. Geological Survey; ID, identification number; $\mathrm{dd}^{\circ} \mathrm{mm}$ 'ss", degrees, minutes, seconds; $\mathrm{mi}^{2}$, square mile; $\mathrm{ft}^{3} / \mathrm{s} / \mathrm{mi}^{2}$, cubic foot per second per square mile; $\mathrm{ft}^{3} / \mathrm{s}$, cubic foot per second; equation, statewide regression equation; relation, established relation line with index station; R, River; Bk, Brook; Br, Branch; Fk, Fork; Ck, Creek; Tr, Tributary; nr, near; N, North; S, South; E, East; W, West; ,-- not determined in historical low-flow reports. See references for full list of low-flow reports.]

\begin{tabular}{|c|c|c|c|c|c|c|c|c|c|c|}
\hline $\begin{array}{c}\text { Map } \\
\text { number }\end{array}$ & $\begin{array}{c}\text { USGS } \\
\text { station ID }\end{array}$ & Station name & $\begin{array}{c}\text { Latitude } \\
\text { (dd }{ }^{\circ} \mathrm{mm} \text { 'ss") }\end{array}$ & $\begin{array}{l}\text { Longitude } \\
\text { (ddºmm'ss") }\end{array}$ & $\begin{array}{c}\text { Index } \\
\text { station }^{\text {a }}\end{array}$ & $\begin{array}{c}\text { Drainage } \\
\text { area } \\
\left(\mathrm{mi}^{2}\right)\end{array}$ & $\begin{array}{c}\text { Baseflow } \\
\text { factor } \\
\left(\mathrm{ft}^{3} / \mathrm{s} / \mathrm{mi}^{2}\right)\end{array}$ & $\begin{array}{c}\text { Baseflow } \\
\text { method }\end{array}$ & $\begin{array}{c}\text { Baseflow } \\
\quad\left(\mathrm{ft}^{3} / \mathrm{s}\right)\end{array}$ & $\begin{array}{c}\text { Recharge } \\
\text { (inches) }\end{array}$ \\
\hline 1 & 05390140 & Muskrat Ck at Conover & $46^{\circ} 03^{\prime} 27^{\prime \prime}$ & $89^{\circ} 15^{\prime} 24^{\prime \prime}$ & 04061000 & 10.2 & -- & relation & 5.1 & 6.8 \\
\hline 2 & 05390170 & Pioneer $\mathrm{Ck}$ at Conover & $46^{\circ} 03^{\prime} 14^{\prime \prime}$ & $89^{\circ} 15^{\prime} 27^{\prime \prime}$ & 04061000 & 42 & -- & relation & 18.5 & 6.0 \\
\hline 3 & 05390450 & Deerskin R nr Eagle R & $46^{\circ} 00^{\prime} 11^{\prime \prime}$ & $89^{\circ} 04^{\prime} 30^{\prime \prime}$ & 04061000 & 32.4 & -- & relation & 20.0 & 8.4 \\
\hline 4 & 05390619 & Rice Ck nr Eagle R & $45^{\circ} 56^{\prime} 28^{\prime \prime}$ & $89^{\circ} 19^{\prime} 27^{\prime \prime}$ & 04061000 & 26.5 & -- & relation & 15.5 & 7.9 \\
\hline 5 & 05390725 & Plum Ck nr Sayner & $45^{\circ} 59^{\prime} 23^{\prime \prime}$ & $89^{\circ} 33^{\prime} 14^{\prime \prime}$ & 04061000 & 31.6 & -- & relation & 20.5 & 8.8 \\
\hline 6 & 05390741 & Stella Ck nr Sayner & $45^{\circ} 59^{\prime} 02^{\prime \prime}$ & $89^{\circ} 28^{\prime} 02^{\prime \prime}$ & 04061000 & 6.22 & -- & relation & 4.3 & 9.4 \\
\hline 7 & 05391028 & Horsehead Ck nr Lk Tomahawk & $45^{\circ} 46^{\prime} 17^{\prime \prime}$ & $89^{\circ} 35^{\prime} 23^{\prime \prime}$ & 05394500 & 5.58 & 0.3565 & equation & 3.1 & 7.5 \\
\hline 8 & 05391042 & Tom Doyle Ck nr Mc Naughton & $45^{\circ} 43^{\prime} 57^{\prime \prime}$ & $89^{\circ} 30^{\prime} 29^{\prime \prime}$ & 05394500 & 10.2 & .2925 & equation & 5.1 & 6.8 \\
\hline 9 & 05391055 & Newbold Ck nr Rhinelander & $45^{\circ} 41^{\prime} 24^{\prime \prime}$ & $89^{\circ} 30^{\prime} 20^{\prime \prime}$ & 05394500 & 10.9 & .2062 & equation & 4.6 & 5.7 \\
\hline 10 & 05391069 & Skunk Ck nr Rhinelander & $45^{\circ} 41^{\prime} 47^{\prime \prime}$ & $89^{\circ} 27^{\prime} 04^{\prime \prime}$ & 05394500 & 7.3 & .1249 & equation & 2.3 & 4.3 \\
\hline 11 & 05391085 & N Pine Lk Ck nr Rhinelander & $45^{\circ} 44^{\prime} 14^{\prime \prime}$ & $89^{\circ} 22^{\prime} 05^{\prime \prime}$ & 04074950 & 14 & .1290 & relation & 4.4 & 4.3 \\
\hline 12 & 05391088 & Pine Lk Ck nr Rhinelander & $45^{\circ} 41^{\prime} 44^{\prime \prime}$ & $89^{\circ} 23^{\prime} 04^{\prime \prime}$ & 05394500 & 26.3 & .0203 & equation & 3.4 & 1.7 \\
\hline 13 & 05391200 & Monico $\mathrm{Ck}$ at Monico & $45^{\circ} 34^{\prime} 39^{\prime \prime}$ & $89^{\circ} 08^{\prime} 57^{\prime \prime}$ & 04074950 & 20.8 & .0090 & relation & 1.5 & 1.0 \\
\hline 14 & 05391223 & Neptune Ck nr Monico & $45^{\circ} 35^{\prime} 04^{\prime \prime}$ & $89^{\circ} 14^{\prime} 08^{\prime \prime}$ & 04074950 & 9.04 & .0526 & equation & 1.9 & 2.8 \\
\hline 15 & 05391250 & Gudegast Ck nr Rhinelander & $45^{\circ} 42^{\prime} 27^{\prime \prime}$ & $89^{\circ} 14^{\prime} 40^{\prime \prime}$ & 04074950 & 12.4 & .0620 & relation & 5.6 & 6.1 \\
\hline 16 & 05391265 & Lost Ck nr Sugar Camp & $45^{\circ} 45^{\prime} 00^{\prime \prime}$ & $89^{\circ} 19^{\prime} 16^{\prime \prime}$ & 04074950 & 12.7 & -- & relation & 4.0 & 4.3 \\
\hline 17 & 05391269 & Jennie Webber Ck nr Rhinelander & $45^{\circ} 43^{\prime} 20^{\prime \prime}$ & $89^{\circ} 18^{\prime} 57^{\prime \prime}$ & 04074950 & 18.9 & .2794 & equation & 9.4 & 6.7 \\
\hline 18 & 05391278 & Starks Ck nr Rhinelander & $45^{\circ} 40^{\prime} 08^{\prime \prime}$ & $89^{\circ} 15^{\prime} 06^{\prime \prime}$ & 04074950 & 8.59 & .6901 & equation & 6.7 & 10.6 \\
\hline 19 & 053913035 & Haymeadow Ck nr Rhinelander & $45^{\circ} 35^{\prime} 40^{\prime \prime}$ & $89^{\circ} 15^{\prime} 55^{\prime \prime}$ & 04074950 & 20.5 & .0103 & equation & 1.8 & 1.2 \\
\hline 20 & 05391350 & Lk George Ck nr Rhinelander & $45^{\circ} 36^{\prime} 00^{\prime \prime}$ & $89^{\circ} 18^{\prime} 42^{\prime \prime}$ & 05394500 & 11.9 & .1289 & equation & 3.9 & 4.5 \\
\hline 21 & 05391360 & Bergman Ck nr Rhinelander & $45^{\circ} 34^{\prime} 44^{\prime \prime}$ & $89^{\circ} 21^{\prime} 23^{\prime \prime}$ & 05394500 & 5.48 & .2370 & equation & 2.4 & 6.0 \\
\hline 22 & 05391450 & Pelican R at Rhinelander & $45^{\circ} 37^{\prime} 43^{\prime \prime}$ & $89^{\circ} 25^{\prime} 21^{\prime \prime}$ & 05394500 & 264 & -- & relation & 130.0 & 6.7 \\
\hline 23 & 05391900 & Noisy Ck nr Rhinelander & $45^{\circ} 33^{\prime} 21^{\prime \prime}$ & $89^{\circ} 26^{\prime} 54^{\prime \prime}$ & 05394500 & 35.6 & .1820 & relation & 17.5 & 6.7 \\
\hline
\end{tabular}

${ }^{1}$ Low-flow discharge measurements at partial-record stations were compared to one or more nearby USGS gaging stations to establish a relation between measured discharge and concurrent daily discharge. The nearby gaging station that provided the best relation with the partial-record station was chosen as the index station and was associated with nearby miscellaneous measurement sites.

${ }^{2}$ Mismatch between surface-water and ground-water drainage area.

${ }^{3}$ Recharge likely altered by regulation. 
Appendix 4D. Average annual base flow and corresponding recharge, 1970-99, for partial-record stations in the Upper Wisconsin River Basin-Continued.

[USGS, U.S. Geological Survey; ID, identification number; $\mathrm{dd}^{\circ} \mathrm{mm}$ 'ss", degrees, minutes, seconds; $\mathrm{mi}^{2}$, square mile; $\mathrm{ft}^{3} / \mathrm{s} / \mathrm{mi}^{2}$, cubic foot per second per square mile; $\mathrm{ft}^{3} / \mathrm{s}$, cubic foot per second; equation, statewide regression equation; relation, established relation line with index station; R, River; Bk, Brook; Br, Branch; Fk, Fork; Ck, Creek; Tr, Tributary; nr, near; N, North; S, South; E, East; W, West; --, not determined in historical low-flow reports. See references for full list of low-flow reports.]

\begin{tabular}{|c|c|c|c|c|c|c|c|c|c|c|}
\hline $\begin{array}{c}\text { Map } \\
\text { number }\end{array}$ & $\begin{array}{c}\text { USGS } \\
\text { station ID }\end{array}$ & Station name & $\begin{array}{c}\text { Latitude } \\
\text { (ddºmm'ss") }\end{array}$ & $\begin{array}{l}\text { Longitude } \\
\text { (ddºmm'ss") }\end{array}$ & $\begin{array}{c}\text { Index } \\
\text { station }^{\mathrm{a}}\end{array}$ & $\begin{array}{c}\text { Drainage } \\
\text { area } \\
\left(\mathrm{mi}^{2}\right)\end{array}$ & $\begin{array}{c}\text { Baseflow } \\
\text { factor } \\
\left(\mathrm{ft}^{3} / \mathrm{s} / \mathrm{mi}^{2}\right)\end{array}$ & $\begin{array}{l}\text { Baseflow } \\
\text { method }\end{array}$ & $\begin{array}{c}\text { Baseflow } \\
\left(\mathrm{ft}^{3} / \mathrm{s}\right)\end{array}$ & $\begin{array}{c}\text { Recharge } \\
\text { (inches) }\end{array}$ \\
\hline 24 & 05391958 & Crescent Ck nr Woodboro & $45^{\circ} 34^{\prime} 37^{\prime \prime}$ & $89^{\circ} 30^{\prime} 35^{\prime \prime}$ & 05394500 & 14.8 & 0.0809 & equation & 3.8 & 3.5 \\
\hline 25 & 05392020 & Big Pine Ck nr Harrison & $45^{\circ} 28^{\prime} 56^{\prime \prime}$ & $89^{\circ} 34^{\prime} 30^{\prime \prime}$ & 05394500 & 29.4 & .3638 & equation & 16.9 & 7.8 \\
\hline 26 & 05392031 & Muskelunge Ck nr Tomahawk & $45^{\circ} 29^{\prime} 48^{\prime \prime}$ & $89^{\circ} 42^{\prime} 29^{\prime \prime}$ & 05394500 & 8.18 & .3238 & equation & 4.3 & 7.1 \\
\hline 27 & 05392102 & Tomahawk R nr Minocqua & $45^{\circ} 53^{\prime} 00^{\prime \prime}$ & $89^{\circ} 44^{\prime} 32^{\prime \prime}$ & 05394500 & 73 & .1135 & equation & 23.3 & 4.3 \\
\hline 28 & 05392150 & Mishonagon Ck nr Woodruff & $45^{\circ} 54^{\prime} 41^{\prime \prime}$ & $89^{\circ} 45^{\prime} 30^{\prime \prime}$ & 04061000 & 17.6 & 1.4130 & relation & 11.5 & 8.9 \\
\hline 29 & 05392202 & Squirrel R nr Minocqua & $45^{\circ} 50^{\prime} 25^{\prime \prime}$ & $89^{\circ} 54^{\prime} 08^{\prime \prime}$ & 05393500 & 15.5 & 1.8020 & equation & 20.2 & $17.7^{\mathrm{c}}$ \\
\hline 30 & 05392220 & Tomahawk R nr Hazelhurst & $45^{\circ} 48^{\prime} 40^{\prime \prime}$ & $89^{\circ} 49^{\prime} 05^{\prime \prime}$ & 05394500 & 165 & .3966 & equation & 102.5 & 8.4 \\
\hline 31 & 05392227 & Threemile Ck nr Hazelhurst & $45^{\circ} 48^{\prime} 57^{\prime \prime}$ & $89^{\circ} 46^{\prime} 22^{\prime \prime}$ & 05394500 & 4.97 & .0737 & equation & 1.2 & 3.3 \\
\hline 32 & 05392233 & Kaubashine Ck nr Hazelhurst & $45^{\circ} 47^{\prime} 59^{\prime \prime}$ & $89^{\circ} 48^{\prime} 04^{\prime \prime}$ & 05394500 & 18.4 & .5758 & equation & 13.3 & 9.8 \\
\hline 33 & 05392240 & Tomahawk R nr Hazelhurst & $45^{\circ} 45^{\prime} 31^{\prime \prime}$ & $89^{\circ} 50^{\prime} 44^{\prime \prime}$ & 05394500 & 189 & .3989 & equation & 118.0 & 8.5 \\
\hline 34 & 05392290 & Willow R nr Hazelhurst & $45^{\circ} 45^{\prime} 37^{\prime \prime}$ & $90^{\circ} 01^{\prime} 28^{\prime \prime}$ & 05394500 & 9.92 & .4350 & relation & 6.0 & 8.2 \\
\hline 35 & 053922926 & Thunder R nr Tripoli & $45^{\circ} 41^{\prime} 56^{\prime \prime}$ & $90^{\circ} 01^{\prime} 19^{\prime \prime}$ & 05394500 & 11.8 & .1124 & equation & 3.6 & 4.2 \\
\hline 36 & 05392298 & Swamsauger Ck nr Hazelhurst & $45^{\circ} 43^{\prime} 55^{\prime \prime}$ & $89^{\circ} 55^{\prime} 54^{\prime \prime}$ & 05394500 & 15.8 & .1867 & equation & 6.3 & 5.4 \\
\hline 37 & 05392305 & Bear Ck nr Goodnow & $45^{\circ} 42^{\prime} 37^{\prime \prime}$ & $89^{\circ} 48^{\prime} 10^{\prime \prime}$ & 05394500 & 6.2 & .5407 & equation & 4.2 & 9.3 \\
\hline 38 & 05392320 & Rocky Run Ck nr Goodnow & $45^{\circ} 41^{\prime} 37^{\prime \prime}$ & $89^{\circ} 45^{\prime} 31^{\prime \prime}$ & 05394500 & 23.2 & .8660 & relation & 23.5 & 13.8 \\
\hline 39 & 05392350 & Bearskin Ck nr Harshaw & $45^{\circ} 38^{\prime} 43^{\prime \prime}$ & $89^{\circ} 41^{\prime} 12^{\prime \prime}$ & 05393500 & 31.1 & 1.1680 & relation & 36.5 & $15.9^{\mathrm{b}}$ \\
\hline 40 & 05392378 & Swamp Ck nr Bradley & $45^{\circ} 37^{\prime} 08^{\prime \prime}$ & $89^{\circ} 45^{\prime} 03^{\prime \prime}$ & 05394500 & 11.7 & .3778 & equation & 6.7 & 7.8 \\
\hline 41 & 05392405 & Rice Ck nr Harshaw & $45^{\circ} 38^{\prime} 36^{\prime \prime}$ & $89^{\circ} 36^{\prime} 21^{\prime \prime}$ & 05394500 & 18.1 & .4508 & equation & 11.5 & 8.6 \\
\hline 42 & 05392407 & Trout Ck nr Woodboro & $45^{\circ} 36^{\prime} 26^{\prime \prime}$ & $89^{\circ} 36^{\prime} 13^{\prime \prime}$ & 05394500 & 2.22 & 2.1841 & equation & 3.1 & $18.8^{\mathrm{b}}$ \\
\hline 43 & 05392410 & Little Rice Ck nr Haefford Junction & $45^{\circ} 34^{\prime} 00^{\prime \prime}$ & $89^{\circ} 40^{\prime} 15^{\prime \prime}$ & 05394500 & 36.9 & .5867 & equation & 27.3 & 10.0 \\
\hline 44 & 05392450 & Little Rice R nr Bradley & $45^{\circ} 35^{\prime} 43^{\prime \prime}$ & $89^{\circ} 49^{\prime} 28^{\prime \prime}$ & 05394500 & 21.4 & .0890 & relation & 15.1 & 9.6 \\
\hline 45 & 05392460 & Brown Ck nr Mccord & $45^{\circ} 38^{\prime} 18^{\prime \prime}$ & $89^{\circ} 48^{\prime} 58^{\prime \prime}$ & 05394500 & 7.49 & .0824 & equation & 1.9 & 3.5 \\
\hline 46 & 05393160 & Somo Ck at Clifford & $45^{\circ} 33^{\prime} 17^{\prime \prime}$ & $90^{\circ} 02^{\prime} 36^{\prime \prime}$ & 05393500 & 18.8 & .0409 & equation & 3.4 & 2.5 \\
\hline
\end{tabular}

${ }^{1}$ Low-flow discharge measurements at partial-record stations were compared to one or more nearby USGS gaging stations to establish a relation between measured discharge and concurrent daily discharge. The nearby gaging station that provided the best relation with the partial-record station was chosen as the index station and was associated with nearby miscellaneous measurement sites.

${ }^{2}$ Mismatch between surface-water and ground-water drainage area.

${ }^{3}$ Recharge likely altered by regulation. 
[USGS, U.S. Geological Survey; ID, identification number; $\mathrm{dd}^{\circ} \mathrm{mm}$ 'ss", degrees, minutes, seconds; $\mathrm{mi}^{2}$, square mile; $\mathrm{ft}^{3} / \mathrm{s} / \mathrm{mi}^{2}$, cubic foot per second per square mile; $\mathrm{ft}^{3} / \mathrm{s}$, cubic foot per second; equation, statewide regression equation; relation, established relation line with index station; R, River; Bk, Brook; Br, Branch; Fk, Fork; Ck, Creek; Tr, Tributary; nr, near; N, North; S, South; E, East; W, West;

--, not determined in historical low-flow reports. See references for full list of low-flow reports.]

\begin{tabular}{|c|c|c|c|c|c|c|c|c|c|c|}
\hline $\begin{array}{c}\text { Map } \\
\text { number }\end{array}$ & $\begin{array}{c}\text { USGS } \\
\text { station ID }\end{array}$ & Station name & $\begin{array}{c}\text { Latitude } \\
\text { (dd }{ }^{\circ} \mathrm{mm} \text { 'ss") }\end{array}$ & $\begin{array}{c}\text { Longitude } \\
\text { (ddºmm'ss") }\end{array}$ & $\begin{array}{c}\text { Index } \\
\text { station }^{\text {a }}\end{array}$ & $\begin{array}{c}\text { Drainage } \\
\text { area } \\
\left(\mathrm{mi}^{2}\right)\end{array}$ & $\begin{array}{c}\text { Baseflow } \\
\text { factor } \\
\left(\mathrm{ft}^{3} / \mathrm{s} / \mathrm{mi}^{2}\right)\end{array}$ & $\begin{array}{l}\text { Baseflow } \\
\text { method }\end{array}$ & $\begin{array}{c}\text { Baseflow } \\
\quad\left(\mathrm{ft}^{3} / \mathrm{s}\right)\end{array}$ & $\begin{array}{c}\text { Recharge } \\
\text { (inches) }\end{array}$ \\
\hline 47 & 05393194 & Scott Ck at Tripoli & $45^{\circ} 33^{\prime} 44^{\prime \prime}$ & $90^{\circ} 00^{\prime} 27^{\prime \prime}$ & 05393500 & 11.3 & 0.0885 & equation & 3.0 & 3.7 \\
\hline 48 & 05393200 & Somo R nr Tripoli & $45^{\circ} 32^{\prime} 20^{\prime \prime}$ & $89^{\circ} 58^{\prime} 10^{\prime \prime}$ & 05393500 & 43.5 & .1230 & relation & 12.0 & 3.7 \\
\hline 49 & 05393360 & Johnson Ck nr Tomahawk & $45^{\circ} 31^{\prime} 33^{\prime \prime}$ & $89^{\circ} 52^{\prime} 03^{\prime \prime}$ & 05393500 & 6.41 & .0588 & equation & 1.4 & 2.9 \\
\hline 50 & 05393380 & Hay Ck nr Tomahawk & $45^{\circ} 30^{\prime} 39^{\prime \prime}$ & $89^{\circ} 49^{\prime} 17^{\prime \prime}$ & 05393500 & 3.99 & .0218 & equation & .5 & 1.7 \\
\hline 51 & 05393402 & Spirit R nr Spirit & $45^{\circ} 24^{\prime} 01^{\prime \prime}$ & $90^{\circ} 05^{\prime} 22^{\prime \prime}$ & 05393500 & 22 & .1943 & equation & 9.1 & 5.6 \\
\hline 52 & 05393440 & N Fk Spirit R nr Spirit & $45^{\circ} 28^{\prime} 04^{\prime \prime}$ & $90^{\circ} 05^{\prime} 38^{\prime \prime}$ & 05393500 & 10.5 & .0718 & equation & 2.5 & 3.3 \\
\hline 53 & 05393450 & Knox Ck nr Spirit & $45^{\circ} 29^{\prime} 34^{\prime \prime}$ & $90^{\circ} 06^{\prime} 16^{\prime \prime}$ & 05393500 & 7.07 & .0369 & equation & 1.2 & 2.3 \\
\hline 54 & 05393460 & N Fk Spirit R nr Spirit & $45^{\circ} 27^{\prime} 14^{\prime \prime}$ & $90^{\circ} 02^{\prime} 24^{\prime \prime}$ & 05393500 & 22.8 & .0559 & equation & 4.9 & 2.9 \\
\hline 55 & 05393480 & Ritchie Ck nr Spirit & $45^{\circ} 27^{\prime} 11^{\prime \prime}$ & $90^{\circ} 01^{\prime} 18^{\prime \prime}$ & 05393500 & 7.15 & .1115 & equation & 2.2 & 4.1 \\
\hline 56 & 05393520 & Squaw Ck at Spirit Falls & $45^{\circ} 27^{\prime} 23^{\prime \prime}$ & $89^{\circ} 58^{\prime} 27^{\prime \prime}$ & 05393500 & 19.7 & .1010 & relation & 5.4 & 3.7 \\
\hline 57 & 05393560 & Armstrong Ck nr Tomahawk & $45^{\circ} 26^{\prime} 23^{\prime \prime}$ & $89^{\circ} 50^{\prime} 58^{\prime \prime}$ & 05393500 & 13 & .0410 & relation & 4.5 & 4.7 \\
\hline 58 & 05393572 & Coffee Ck nr Tomahawk & $45^{\circ} 25^{\prime} 29^{\prime \prime}$ & $89^{\circ} 48^{\prime} 30^{\prime \prime}$ & 05393500 & 4.68 & .0372 & equation & .8 & 2.3 \\
\hline
\end{tabular}

${ }^{1}$ Low-flow discharge measurements at partial-record stations were compared to one or more nearby USGS gaging stations to establish a relation between measured discharge and concurrent daily discharge. The nearby gaging station that provided the best relation with the partial-record station was chosen as the index station and was associated with nearby miscellaneous measurement sites.

${ }^{2}$ Mismatch between surface-water and ground-water drainage area.

${ }^{3}$ Recharge likely altered by regulation. 


\section{Menominee-Oconto-Peshtigo River Basin}

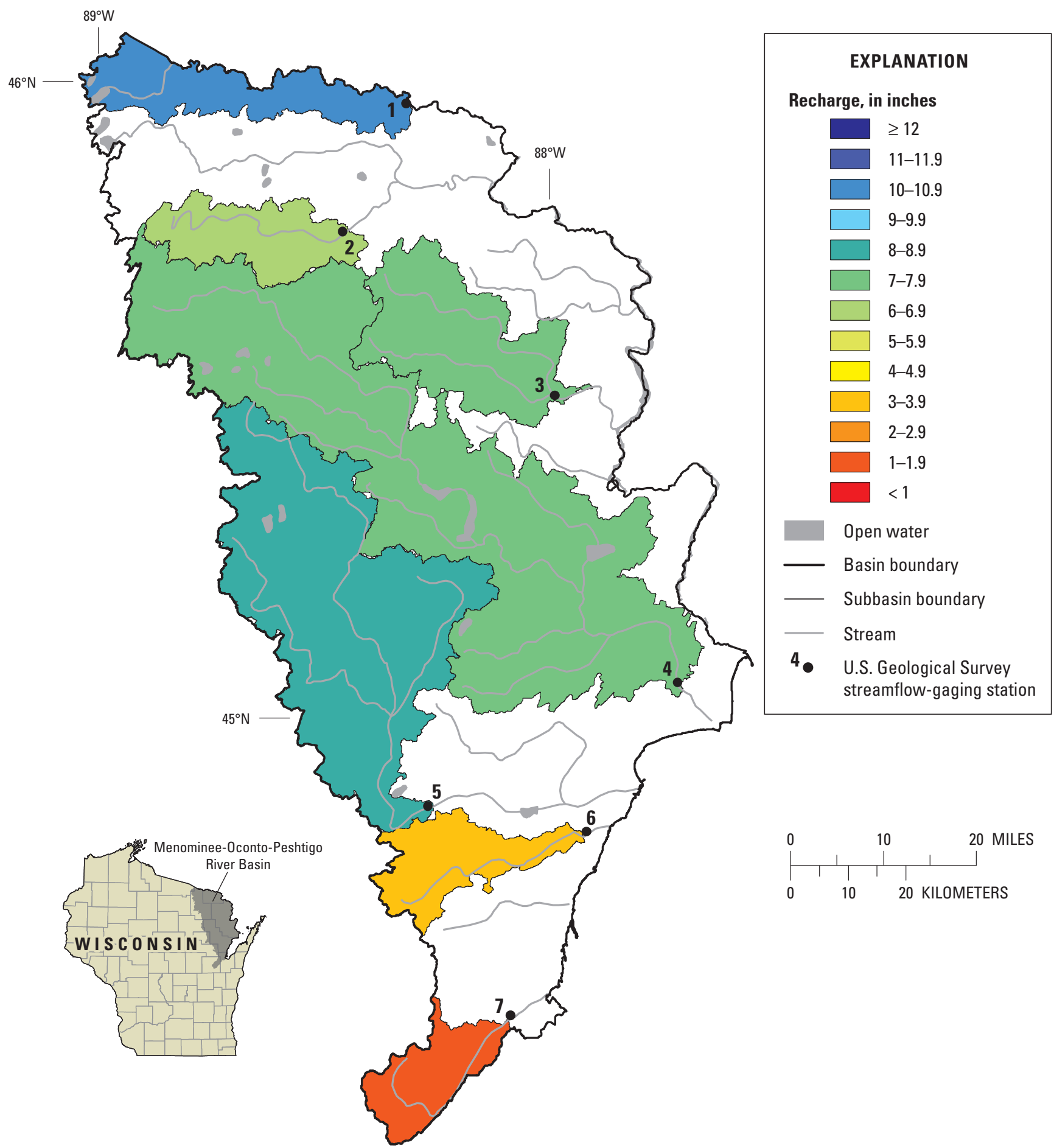

Appendix 5-A. Locations of streamflow-gaging stations in the Menominee-0conto-Peshtigo River Basin and spatial distribution of average annual recharge. 


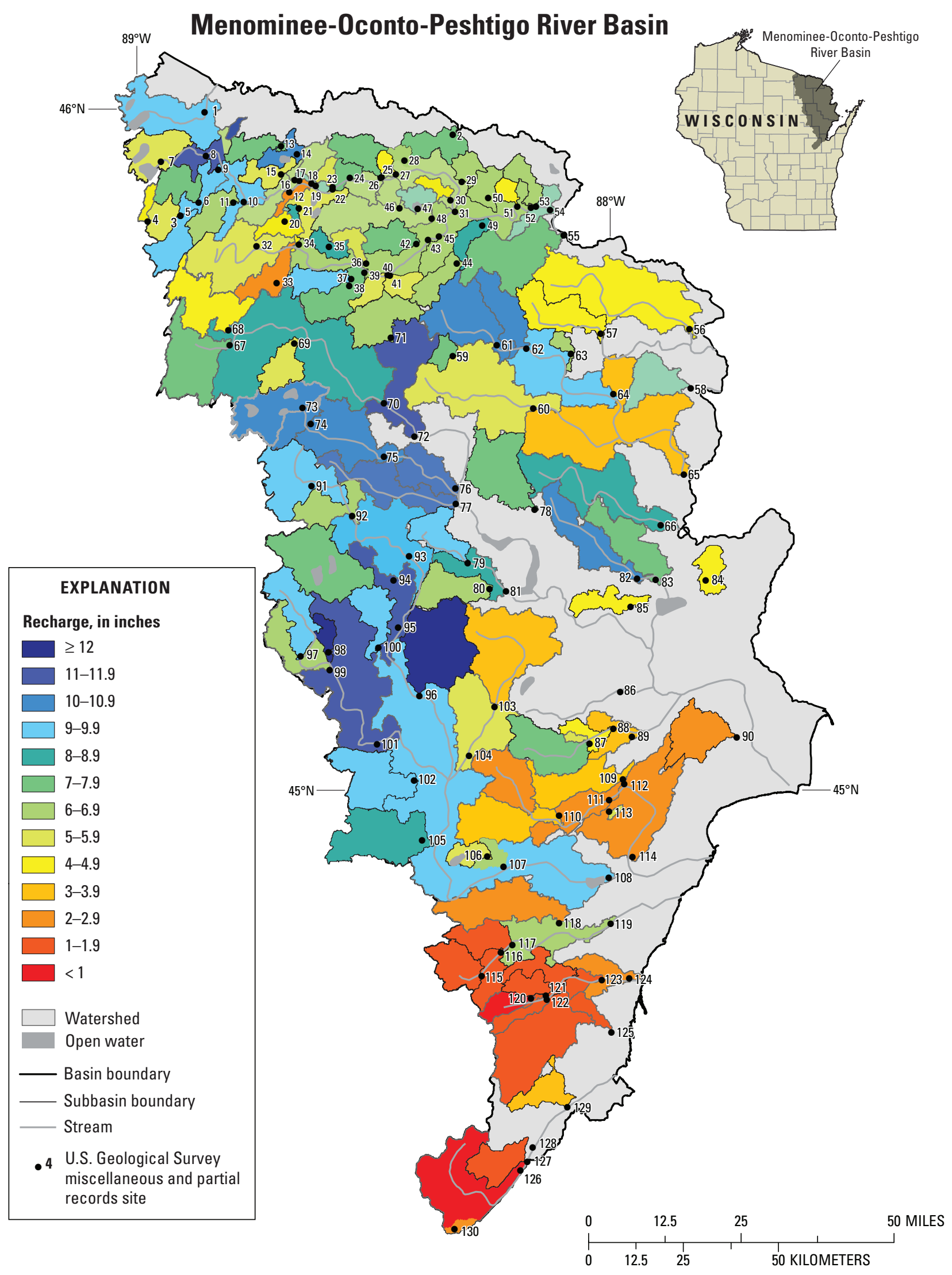

Appendix 5-B. Locations of miscellaneous and partial-record sites in the Menominee-0conto-Peshtigo River Basin and spatial distribution of estimated average annual recharge. 
Appendix 5C. Average annual base flow and corresponding recharge, 1970-99, for streamflow-gaging stations in the Menominee-0conto-Peshtigo River Basin.

[USGS, U.S. Geological Survey; ID, identification number; mi², square mile; $\mathrm{ft}^{3}$ /s, cubic foot per second; BFI, Baseflow Index; ex rec, extended record; R, River; Bk, Brook; Br, Branch; Fk, Fork; Ck, Creek; Tr, Tributary; nr, near; N, North; S, South; E, East; W, West; --, not determined in historical low-flow reports. See references for full list of low-flow reports.]

\begin{tabular}{|c|c|c|c|c|c|c|c|c|c|c|c|}
\hline $\begin{array}{c}\text { Map } \\
\text { number }\end{array}$ & $\begin{array}{c}\text { USGS } \\
\text { station } \\
\text { ID }\end{array}$ & Station name & $\begin{array}{c}\text { Drainage } \\
\text { area } \\
\left(\mathrm{mi}^{2}\right)\end{array}$ & $\begin{array}{c}\text { Period of } \\
\text { analysis } \\
\text { (water years }{ }^{1} \text { ) }\end{array}$ & $\begin{array}{c}\text { Base flow } \\
\text { method }\end{array}$ & $\begin{array}{l}\text { Base flow } \\
\text { index }\end{array}$ & $\begin{array}{l}\text { Base } \\
\text { flow } \\
\left(\mathrm{ft}^{3} / \mathrm{s}\right)\end{array}$ & $\begin{array}{c}\text { Recharge } \\
\text { (inches) }\end{array}$ & $\begin{array}{c}\text { Total } \\
\text { streamflow } \\
\left(\mathrm{ft}^{3} / \mathbf{s}\right)\end{array}$ & $\begin{array}{c}\text { Total } \\
\text { streamflow } \\
\text { (inches) }\end{array}$ & $\begin{array}{l}{ }^{2} 090 \\
\left(\mathrm{ft}^{3} / \mathrm{s}\right)\end{array}$ \\
\hline 1 & 04060993 & Brule R nr Florence & 366 & $1970-1999$ & BFI & 0.8130 & 280 & 10.5 & 350 & 13.1 & 120 \\
\hline 2 & 04063700 & Popple R nr Fence & 139 & 1970-1999 & BFI & .5980 & 68 & 6.6 & 120 & 11.2 & 33 \\
\hline 3 & 04066500 & Pike $\mathrm{R}$ at Amberg & 255 & $1914-1970$ & ex rec & -- & 140 & 7.5 & -- & -- & 98 \\
\hline 4 & 04069500 & Peshtigo R at Peshtigo & 1,080 & 1970-1999 & BFI & .6350 & 600 & 7.6 & 950 & 12.0 & 370 \\
\hline 5 & 04071000 & Oconto R nr Gillett & 705 & 1970-1999 & BFI & .7710 & 450 & 8.7 & 590 & 11.3 & 276 \\
\hline 6 & 04071858 & Pensaukee R nr Pensaukee & 134 & 1973-1996 & BFI & .3370 & 31 & 3.1 & 92 & 9.3 & 6.3 \\
\hline 7 & 04072150 & Duck Ck nr Howard & 108 & 1970-1999 & ex rec & -- & 12 & 1.5 & -- & -- & -- \\
\hline
\end{tabular}

${ }^{1}$ Water year is a 12-month period from October 1 through September 30, and is designated by the calendar year in which it ends (for example, the 2004 water year occurred October 1,2003 through September 30, 2004).

${ }^{2}$ Discharge that is exceeded 90 percent of the time. 
[USGS, U.S. Geological Survey; ID, identification number; $\mathrm{dd}^{\circ} \mathrm{mm}$ 'ss", degrees, minutes, seconds; $\mathrm{mi}^{2}$, square mile; $\mathrm{ft}^{3} / \mathrm{s} / \mathrm{mi}^{2}$, cubic foot per second per square mile; $\mathrm{ft}^{3} / \mathrm{s}$, cubic foot per second; equation, statewide regression equation; relation, established relation line with index station; R, River; Bk, Brook; Br, Branch; Fk, Fork; Ck, Creek; Tr, Tributary; nr, near; N, North; S, South; E, East; W, West;

--, not determined in historical low-flow reports. See references for full list of low-flow reports.]

\begin{tabular}{|c|c|c|c|c|c|c|c|c|c|c|}
\hline $\begin{array}{c}\text { Map } \\
\text { number }\end{array}$ & $\begin{array}{c}\text { USGS } \\
\text { station ID }\end{array}$ & Station name & $\begin{array}{c}\text { Latitude } \\
\text { (dd }{ }^{\circ} \mathrm{mm} \text { 'ss") }\end{array}$ & $\begin{array}{l}\text { Longitude } \\
\text { (ddºmm'ss") }\end{array}$ & $\begin{array}{l}\text { Index } \\
\text { station }^{1}\end{array}$ & $\begin{array}{c}\text { Drainage } \\
\text { area } \\
\left(\mathrm{mi}^{2}\right)\end{array}$ & $\begin{array}{l}\text { Base flow } \\
\text { factor } \\
\left(\mathrm{ft}^{3} / \mathrm{s} / \mathrm{mi}^{2}\right)\end{array}$ & $\begin{array}{l}\text { Base flow } \\
\text { method }\end{array}$ & $\begin{array}{c}\text { Base flow } \\
\left(\mathrm{ft}^{3} / \mathrm{s}\right)\end{array}$ & $\begin{array}{c}\text { Recharge } \\
\text { (inches) }\end{array}$ \\
\hline 1 & 04059800 & Brule Ck nr Alvin & $45^{\circ} 59^{\prime} 36^{\prime \prime}$ & $88^{\circ} 51^{\prime} 44^{\prime \prime}$ & 04063700 & 35.6 & 0.4800 & relation & 26.0 & 9.9 \\
\hline 2 & 04060990 & Montagne Ck nr Florence & $45^{\circ} 57^{\prime} 13^{\prime \prime}$ & $88^{\circ} 20^{\prime} 25^{\prime \prime}$ & 04063700 & 14.6 & .3000 & relation & 7.8 & 7.3 \\
\hline 3 & 040636005 & Pine R above Kimball Ck & $45^{\circ} 50^{\prime} 30^{\prime \prime}$ & $88^{\circ} 54^{\prime} 56^{\prime \prime}$ & 04063700 & 15.3 & .1078 & equation & 4.6 & 4.1 \\
\hline 4 & 04063602 & Kimball Ck nr Three Lks & $45^{\circ} 50^{\prime} 01^{\prime \prime}$ & $88^{\circ} 59^{\prime} 03^{\prime \prime}$ & 04063700 & 3.09 & .1654 & equation & 1.1 & 4.9 \\
\hline 5 & 04063604 & Kimball Ck at Mouth nr Three Lks & $45^{\circ} 50^{\prime} 32^{\prime \prime}$ & $88^{\circ} 54^{\prime} 56^{\prime \prime}$ & 04063700 & 13.2 & .1645 & equation & 4.9 & 5.1 \\
\hline 6 & 04063611 & Mcdonald $\mathrm{Ck}$ at Mouth & $45^{\circ} 51^{\prime} 38^{\prime \prime}$ & $88^{\circ} 52^{\prime} 36^{\prime \prime}$ & 04063700 & 13.5 & .3518 & equation & 7.5 & 7.5 \\
\hline 7 & 04063635 & N Br Pine R nr Alvin & $45^{\circ} 54^{\prime} 16^{\prime \prime}$ & $88^{\circ} 52^{\prime} 17^{\prime \prime}$ & 04063700 & 12.6 & .1871 & equation & 5.0 & 5.4 \\
\hline 8 & 04063640 & N Br Pine R nr Windsor Dam & $45^{\circ} 55^{\prime} 43^{\prime \prime}$ & $88^{\circ} 51^{\prime} 38^{\prime \prime}$ & 04063700 & 27.8 & .2530 & relation & 18.0 & 8.8 \\
\hline 9 & 04063643 & Pine R Below N Br nr Alvin & $45^{\circ} 54^{\prime} 32^{\prime \prime}$ & $88^{\circ} 50^{\prime} 05^{\prime \prime}$ & 04063700 & 86 & -- & relation & 62.0 & 9.8 \\
\hline 10 & 04063646 & Pine R nr Alvin & $45^{\circ} 51^{\prime} 39^{\prime \prime}$ & $88^{\circ} 46^{\prime} 55^{\prime \prime}$ & 04063700 & 93.3 & .2675 & equation & 46.7 & 6.8 \\
\hline 11 & 04063648 & Jones Ck nr Alvin & $45^{\circ} 51^{\prime} 34^{\prime \prime}$ & $88^{\circ} 48^{\prime} 16^{\prime \prime}$ & 04063700 & 13.6 & .2298 & equation & 6.0 & 6.0 \\
\hline 12 & 04063653 & Pine R nr Long Lk & $45^{\circ} 52^{\prime} 26^{\prime \prime}$ & $88^{\circ} 41^{\prime} 10^{\prime \prime}$ & 04063700 & 127 & -- & relation & 85.0 & 9.1 \\
\hline 13 & 040636535 & Lilypad Ck nr Tipler & $45^{\circ} 56^{\prime} 29^{\prime \prime}$ & $88^{\circ} 42^{\prime} 06^{\prime \prime}$ & 04063700 & 10 & .3470 & equation & 5.5 & 7.4 \\
\hline 14 & 04063654 & Stevens Ck nr Tipler & $45^{\circ} 55^{\prime} 43^{\prime \prime}$ & $88^{\circ} 40^{\prime} 08^{\prime \prime}$ & 04063700 & 18.2 & -- & relation & 12.0 & 9.0 \\
\hline 15 & 040636545 & Meadowbrook Ck nr Tipler & $45^{\circ} 54^{\prime} 00^{\prime \prime}$ & $88^{\circ} 42^{\prime} 12^{\prime \prime}$ & 04063700 & 8.98 & .2102 & equation & 3.8 & 5.7 \\
\hline 16 & 04063655 & Stevens Ck nr Long Lk & $45^{\circ} 53 \prime 29^{\prime \prime}$ & $88^{\circ} 40^{\prime} 29^{\prime \prime}$ & 04063700 & 30.9 & .2430 & equation & 14.4 & 6.3 \\
\hline 17 & 040636555 & Pine R Tr nr Tipler & $45^{\circ} 53^{\prime} 26^{\prime \prime}$ & $88^{\circ} 39^{\prime} 53^{\prime \prime}$ & 04063700 & 3.97 & .0614 & equation & .9 & 3.0 \\
\hline 18 & 04063656 & Johnson Ck nr Tipler & $45^{\circ} 53^{\prime} 09^{\prime \prime}$ & $88^{\circ} 38^{\prime} 21^{\prime \prime}$ & 04063700 & 7.95 & .2340 & equation & 3.5 & 6.0 \\
\hline 19 & 04063657 & Pine R nr Tipler & $45^{\circ} 52^{\prime} 57^{\prime \prime}$ & $88^{\circ} 37^{\prime} 51^{\prime \prime}$ & 04063700 & 171 & .2783 & equation & 88.4 & 7.0 \\
\hline 20 & 040636575 & Coldwater Ck & $45^{\circ} 49^{\prime} 51^{\prime \prime}$ & $88^{\circ} 41^{\prime} 49^{\prime \prime}$ & 04063700 & 3.59 & .1399 & equation & 1.2 & 4.5 \\
\hline 21 & 04063658 & Long Lk Outlet at Long Lk & $45^{\circ} 50^{\prime} 00^{\prime \prime}$ & $88^{\circ} 40^{\prime} 04^{\prime \prime}$ & 04063700 & 10.1 & .2983 & equation & 5.1 & 6.9 \\
\hline 22 & 04063659 & Fay Lk Outlet nr Tipler & $45^{\circ} 52^{\prime} 37^{\prime \prime}$ & $88^{\circ} 35^{\prime} 42^{\prime \prime}$ & 04063700 & 22.2 & .2327 & equation & 10.0 & 6.1 \\
\hline 23 & 040636595 & Pine R Tr nr Tipler & $45^{\circ} 52^{\prime} 49^{\prime \prime}$ & $88^{\circ} 35^{\prime} 45^{\prime \prime}$ & 04063700 & 4.16 & .1690 & equation & 1.5 & 5.0 \\
\hline 24 & 04063660 & Pine R nr Tipler & $45^{\circ} 53^{\prime} 37^{\prime \prime}$ & $88^{\circ} 33^{\prime} 30^{\prime \prime}$ & 04063700 & 204 & -- & relation & 119.0 & 7.9 \\
\hline
\end{tabular}

${ }^{1}$ Low-flow discharge measurements at partial-record stations were compared to one or more nearby USGS gaging stations to establish a relation between measured discharge and concurrent daily discharge. The nearby gaging station that provided the best relation with the partial-record station was chosen as the index station and was associated with nearby miscellaneous measurement sites.

${ }^{2}$ Mismatch between surface-water and ground-water drainage area. 
Appendix 5D. Average annual base flow and corresponding recharge, 1970-99, for partial-record stations in the Menominee-0conto-Peshtigo River Basin—Continued.

[USGS, U.S. Geological Survey; ID, identification number; $\mathrm{dd}^{\circ} \mathrm{mm}$ 'ss", degrees, minutes, seconds; $\mathrm{mi}^{2}$, square mile; $\mathrm{ft}^{3} / \mathrm{s} / \mathrm{mi}^{2}$, cubic foot per second per square mile; $\mathrm{ft}^{3} / \mathrm{s}$, cubic foot per second; equation, statewide regression equation; relation, established relation line with index station; R, River; Bk, Brook; Br, Branch; Fk, Fork; Ck, Creek; Tr, Tributary; nr, near; N, North; S, South; E, East; W, West; ,-- not determined in historical low-flow reports. See references for full list of low-flow reports.]

\begin{tabular}{|c|c|c|c|c|c|c|c|c|c|c|}
\hline $\begin{array}{c}\text { Map } \\
\text { number }\end{array}$ & $\begin{array}{c}\text { USGS } \\
\text { station ID }\end{array}$ & Station name & $\begin{array}{c}\text { Latitude } \\
\text { (dd }{ }^{\circ} \mathrm{mm} \text { 'ss") }\end{array}$ & $\begin{array}{c}\text { Longitude } \\
\text { (dd }{ }^{\circ} \mathrm{mm} \text { 'ss") }\end{array}$ & $\begin{array}{l}\text { Index } \\
\text { station }{ }^{1}\end{array}$ & $\begin{array}{c}\text { Drainage } \\
\text { area } \\
\left(\mathrm{mi}^{2}\right)\end{array}$ & $\begin{array}{c}\text { Base flow } \\
\text { factor } \\
\left(\mathrm{ft}^{3} / \mathrm{s} / \mathrm{mi}^{2}\right)\end{array}$ & $\begin{array}{c}\text { Base flow } \\
\text { method }\end{array}$ & $\begin{array}{c}\text { Base flow } \\
\qquad\left(\mathrm{ft}^{3} / \mathbf{s}\right)\end{array}$ & $\begin{array}{c}\text { Recharge } \\
\text { (inches) }\end{array}$ \\
\hline 25 & 04063663 & Lautermans Ck nr Tipler & $45^{\circ} 53^{\prime} 36^{\prime \prime}$ & $88^{\circ} 29^{\prime} 30^{\prime \prime}$ & 04063700 & 14.5 & 0.0297 & equation & 2.2 & 2.1 \\
\hline 26 & 04063664 & Pine R nr Tipler & $45^{\circ} 53^{\prime} 34^{\prime \prime}$ & $88^{\circ} 29^{\prime} 38^{\prime \prime}$ & 04063700 & 227 & .2362 & equation & 108.4 & 6.5 \\
\hline 27 & 040636645 & Kieper Ck nr Florence & $45^{\circ} 53^{\prime} 50^{\prime \prime}$ & $88^{\circ} 27^{\prime} 42^{\prime \prime}$ & 04063700 & 3.06 & .1532 & equation & 1.1 & 4.7 \\
\hline 28 & 04063665 & Wakefield Ck nr Florence & $45^{\circ} 55^{\prime} 02^{\prime \prime}$ & $88^{\circ} 26^{\prime} 35^{\prime \prime}$ & 04063700 & 2.58 & .2495 & equation & 1.2 & 6.1 \\
\hline 29 & 04063668 & Pine R Tr nr Florence & $45^{\circ} 53^{\prime} 04^{\prime \prime}$ & $88^{\circ} 19^{\prime} 26^{\prime \prime}$ & 04063700 & 7.43 & .2452 & equation & 3.4 & 6.2 \\
\hline 30 & 04063669 & Seidel Ck nr Fence & $45^{\circ} 51^{\prime} 29^{\prime \prime}$ & $88^{\circ} 20^{\prime} 54^{\prime \prime}$ & 04063700 & 4.37 & .1546 & equation & 1.5 & 4.8 \\
\hline 31 & 04063671 & Pine R nr Florence & $45^{\circ} 50^{\prime} 26^{\prime \prime}$ & $88^{\circ} 20^{\prime} 22^{\prime \prime}$ & 04063700 & 266 & .2365 & equation & 127.5 & 6.5 \\
\hline 32 & 04063672 & Popple R nr Newald & $45^{\circ} 47^{\prime} 45^{\prime \prime}$ & $88^{\circ} 45^{\prime} 26^{\prime \prime}$ & 04063700 & 36.5 & .1703 & equation & 14.2 & 5.3 \\
\hline 33 & 040636725 & Little Popple R at Newald & $45^{\circ} 44^{\prime} 27^{\prime \prime}$ & $88^{\circ} 43^{\prime} 00^{\prime \prime}$ & 04063700 & 9.29 & .0560 & equation & 2.0 & 2.9 \\
\hline 34 & 04063674 & Popple R nr Long Lk & $45^{\circ} 47^{\prime} 47^{\prime \prime}$ & $88^{\circ} 40^{\prime} 05^{\prime \prime}$ & 04063700 & 58.8 & .1407 & equation & 20.9 & 4.8 \\
\hline 35 & 04063677 & Riley Ck nr Long Lk & $45^{\circ} 47^{\prime} 34^{\prime \prime}$ & $88^{\circ} 36^{\prime} 20^{\prime \prime}$ & 04063700 & 2.22 & .4250 & equation & 1.3 & 8.0 \\
\hline 36 & 04063680 & Popple R nr Fence & $45^{\circ} 46^{\prime} 00^{\prime \prime}$ & $88^{\circ} 31^{\prime} 40^{\prime \prime}$ & 04063700 & 85.4 & .1550 & relation & 38.0 & 6.0 \\
\hline 37 & 04063690 & S Br Popple R nr Fence & $45^{\circ} 44^{\prime} 40^{\prime \prime}$ & $88^{\circ} 33^{\prime} 35^{\prime \prime}$ & 04063700 & 12.3 & .3600 & relation & 9.0 & 9.9 \\
\hline 38 & 04063693 & Simpsons Ck nr Fence & $45^{\circ} 44^{\prime} 05^{\prime \prime}$ & $88^{\circ} 33^{\prime} 50^{\prime \prime}$ & 04063700 & 15.4 & .3382 & equation & 8.4 & 7.4 \\
\hline 39 & 04063695 & S Br Popple R nr Fence & $45^{\circ} 45^{\prime} 13^{\prime \prime}$ & $88^{\circ} 31^{\prime} 54^{\prime \prime}$ & 04063700 & 32.1 & .3170 & equation & 17.2 & 7.3 \\
\hline 40 & 04063697 & Rock Ck nr Fence & $45^{\circ} 44^{\prime} 59^{\prime \prime}$ & $88^{\circ} 29^{\prime} 00^{\prime \prime}$ & 04063700 & 6.53 & .3206 & equation & 3.4 & 7.1 \\
\hline 41 & 04063699 & Mud Ck nr Fence & $45^{\circ} 44^{\prime} 58^{\prime \prime}$ & $88^{\circ} 28^{\prime} 00^{\prime \prime}$ & 04063700 & 7.55 & .2090 & equation & 3.2 & 5.7 \\
\hline 42 & 04063710 & Hendricks Ck nr Fence & $45^{\circ} 47^{\prime} 42^{\prime \prime}$ & $88^{\circ} 25^{\prime} 17^{\prime \prime}$ & 04063700 & 13 & .3304 & equation & 7.0 & 7.3 \\
\hline 43 & 04063720 & Popple R nr Florence & $45^{\circ} 48^{\prime} 00^{\prime \prime}$ & $88^{\circ} 23^{\prime} 48^{\prime \prime}$ & 04063700 & 161 & .2357 & equation & 76.2 & 6.4 \\
\hline 44 & 04063740 & Lamon Tangue Ck nr Fence & $45^{\circ} 45^{\prime} 53^{\prime \prime}$ & $88^{\circ} 20^{\prime} 17^{\prime \prime}$ & 04063700 & 14.4 & .2972 & equation & 7.3 & 6.9 \\
\hline 45 & 04063752 & Lamon Tangue Cr nr Fence & $45^{\circ} 48^{\prime} 17^{\prime \prime}$ & $88^{\circ} 22^{\prime} 24^{\prime \prime}$ & 04063700 & 24.1 & .2717 & equation & 11.8 & 6.7 \\
\hline 46 & 04063780 & Haley Cr nr Fence & $45^{\circ} 50^{\prime} 50^{\prime \prime}$ & $88^{\circ} 27^{\prime} 19^{\prime \prime}$ & 04063700 & 5.32 & .2811 & equation & 2.6 & 6.6 \\
\hline 47 & 04063790 & Patten Cr nr Fence & $45^{\circ} 50^{\prime} 46^{\prime \prime}$ & $88^{\circ} 25^{\prime} 02^{\prime \prime}$ & 04063700 & 5.67 & .3183 & equation & 2.9 & 7.0 \\
\hline 48 & 04063800 & Woods Cr nr Fence & $45^{\circ} 49^{\prime} 53^{\prime \prime}$ & $88^{\circ} 23^{\prime} 17^{\prime \prime}$ & 04066500 & 41.9 & .3300 & relation & 20.5 & 6.6 \\
\hline
\end{tabular}

${ }^{1}$ Low-flow discharge measurements at partial-record stations were compared to one or more nearby USGS gaging stations to establish a relation between measured discharge and concurrent daily discharge. The nearby gaging station that provided the best relation with the partial-record station was chosen as the index station and was associated with nearby miscellaneous measurement sites.

${ }^{2}$ Mismatch between surface-water and ground-water drainage area. 
[USGS, U.S. Geological Survey; ID, identification number; $\mathrm{dd}^{\circ} \mathrm{mm}$ 'ss", degrees, minutes, seconds; $\mathrm{mi}^{2}$, square mile; $\mathrm{ft}^{3} / \mathrm{s} / \mathrm{mi}^{2}$, cubic foot per second per square mile; $\mathrm{ft}^{3} / \mathrm{s}$, cubic foot per second; equation, statewide regression equation; relation, established relation line with index station; R, River; Bk, Brook; Br, Branch; Fk, Fork; Ck, Creek; Tr, Tributary; nr, near; N, North; S, South; E, East; W, West; ,-- not determined in historical low-flow reports. See references for full list of low-flow reports.]

\begin{tabular}{|c|c|c|c|c|c|c|c|c|c|c|}
\hline $\begin{array}{c}\text { Map } \\
\text { number }\end{array}$ & $\begin{array}{c}\text { USGS } \\
\text { station ID }\end{array}$ & Station name & $\begin{array}{c}\text { Latitude } \\
\text { (dd }{ }^{\circ} \mathrm{mm} \text { 'ss") }\end{array}$ & $\begin{array}{l}\text { Longitude } \\
\text { (dd }{ }^{\circ} \mathrm{mm} \text { 'ss") }\end{array}$ & $\begin{array}{l}\text { Index } \\
\text { station }{ }^{1}\end{array}$ & $\begin{array}{c}\text { Drainage } \\
\text { area } \\
\left(\mathrm{mi}^{2}\right)\end{array}$ & $\begin{array}{l}\text { Base flow } \\
\text { factor } \\
\left(\mathrm{ft}^{3} / \mathrm{s} / \mathrm{mi}^{2}\right)\end{array}$ & $\begin{array}{c}\text { Base flow } \\
\text { method }\end{array}$ & $\begin{array}{c}\text { Base flow } \\
\quad\left(\mathrm{ft}^{3} / \mathrm{s}\right)\end{array}$ & $\begin{array}{c}\text { Recharge } \\
\text { (inches) }\end{array}$ \\
\hline 49 & 04064200 & Halls Cr nr Florence & $45^{\circ} 49^{\prime} 11^{\prime \prime}$ & $88^{\circ} 16^{\prime} 57^{\prime \prime}$ & 04063700 & 6.7 & 0.4125 & equation & 4.0 & 8.1 \\
\hline 50 & 04064400 & Pine R Tr nr Florence & $45^{\circ} 51^{\prime} 37^{\prime \prime}$ & $88^{\circ} 16^{\prime} 09^{\prime \prime}$ & 04063700 & 7.77 & .2522 & equation & 3.6 & 6.3 \\
\hline 51 & 04064525 & Johnson Cr nr Florence & $45^{\circ} 50^{\prime} 46^{\prime \prime}$ & $88^{\circ} 12^{\prime} 28^{\prime \prime}$ & 04063700 & 4.63 & .1169 & equation & 1.4 & 4.2 \\
\hline 52 & 04064550 & Pine Cr nr Florence & $45^{\circ} 50^{\prime} 39^{\prime \prime}$ & $88^{\circ} 10^{\prime} 46^{\prime \prime}$ & 04063700 & 5.64 & .0763 & equation & 1.4 & 3.3 \\
\hline 53 & 04064580 & Lepage Cr nr Spread Eagle & $45^{\circ} 50^{\prime} 45^{\prime \prime}$ & $88^{\circ} 10^{\prime} 09^{\prime \prime}$ & 04063700 & 9.84 & .2775 & equation & 4.8 & 6.6 \\
\hline 54 & 04064600 & Pine R nr Spread Eagle & $45^{\circ} 50^{\prime} 24^{\prime \prime}$ & $88^{\circ} 08^{\prime} 24^{\prime \prime}$ & 04063700 & 563 & .2693 & equation & 293.0 & 7.1 \\
\hline 55 & 04064900 & Little Popple R at Mouth nr Aurora & $45^{\circ} 48^{\prime} 11^{\prime \prime}$ & $88^{\circ} 06^{\prime} 43^{\prime \prime}$ & 04066500 & 41.3 & .3076 & equation & 21.9 & 7.2 \\
\hline 56 & 04065950 & N Br Pemebonwon R nr Pembine & $45^{\circ} 39^{\prime} 36^{\prime \prime}$ & $87^{\circ} 51^{\prime} 15^{\prime \prime}$ & 04066500 & 77.5 & .1401 & equation & 27.6 & 4.8 \\
\hline 57 & 04065970 & S Br Pemebonwon R nr Pembine & $45^{\circ} 39^{\prime} 23^{\prime \prime}$ & $88^{\circ} 02^{\prime} 25^{\prime \prime}$ & 04066500 & 26.1 & .1360 & relation & 8.5 & 4.4 \\
\hline 58 & 04066015 & Miscauno Ck nr Pembine & $45^{\circ} 34^{\prime} 24^{\prime \prime}$ & $87^{\circ} 51^{\prime} 17^{\prime \prime}$ & 04066500 & 20.4 & .3377 & equation & 11.2 & 7.4 \\
\hline 59 & 04066050 & Chemical $\mathrm{Ck}$ at Goodman & $45^{\circ} 37^{\prime} 43^{\prime \prime}$ & $88^{\circ} 21^{\prime} 00^{\prime \prime}$ & 04066500 & 4.66 & .2500 & relation & 2.5 & 7.3 \\
\hline 60 & 04066250 & S Br Pike R nr Dunbar & $45^{\circ} 32^{\prime} 59^{\prime \prime}$ & $88^{\circ} 11^{\prime} 03^{\prime \prime}$ & 04066500 & 79.9 & -- & relation & 35.0 & 6.0 \\
\hline 61 & 04066280 & N Br Pike R nr Goodman & $45^{\circ} 38^{\prime} 38^{\prime \prime}$ & $88^{\circ} 15^{\prime} 00^{\prime \prime}$ & 04066500 & 33.5 & .6856 & equation & 26.8 & 10.9 \\
\hline 62 & 04066290 & N Br Pike R nr Dunbar & $45^{\circ} 38^{\prime} 16^{\prime \prime}$ & $88^{\circ} 11^{\prime} 00^{\prime \prime}$ & 04066500 & 57.2 & .6588 & equation & 45.3 & 10.8 \\
\hline 63 & 04066300 & Cole Ck nr Dunbar & $45^{\circ} 37^{\prime} 42^{\prime \prime}$ & $88^{\circ} 06^{\prime} 09^{\prime \prime}$ & 04066500 & 3.62 & .1100 & relation & 1.7 & 6.4 \\
\hline 64 & 04066400 & N Br Pike R nr Amberg & $45^{\circ} 34^{\prime} 06^{\prime \prime}$ & $88^{\circ} 01^{\prime} 00^{\prime \prime}$ & 04066500 & 107 & .5584 & equation & 78.7 & 10.0 \\
\hline 65 & 04066550 & Pike R nr Wausaukee & $45^{\circ} 26^{\prime} 49^{\prime \prime}$ & $87^{\circ} 52^{\prime} 25^{\prime \prime}$ & 04066500 & 287 & .3604 & equation & 171.5 & 8.1 \\
\hline 66 & 04066690 & Wausaukee R nr Wausaukee & $45^{\circ} 22^{\prime} 26^{\prime \prime}$ & $87^{\circ} 55^{\prime} 28^{\prime \prime}$ & 04066500 & 52.9 & .3766 & equation & 31.3 & 8.0 \\
\hline 67 & 04067690 & S Br Peshtigo R nr Argonne & $45^{\circ} 39^{\prime} 02^{\prime \prime}$ & $88^{\circ} 49^{\prime} 00^{\prime \prime}$ & 04066500 & 38.5 & .3499 & equation & 21.8 & 7.7 \\
\hline 68 & 04067700 & N Br Peshtigo R nr Argonne & $45^{\circ} 40^{\prime} 22^{\prime \prime}$ & $88^{\circ} 49^{\prime} 07^{\prime \prime}$ & 04066500 & 33.6 & .2300 & relation & 12.0 & 4.9 \\
\hline 69 & 04067750 & Camp Eight Ck nr Cavour & $45^{\circ} 39^{\prime} 06^{\prime \prime}$ & $88^{\circ} 40^{\prime} 52^{\prime \prime}$ & 04066500 & 13.6 & .1940 & relation & 6.0 & 6.0 \\
\hline 70 & 04067790 & Peshtigo R nr Asmstrong Ck & $45^{\circ} 33^{\prime} 44^{\prime \prime}$ & $88^{\circ} 29^{\prime} 44^{\prime \prime}$ & 04066500 & 203 & .3337 & equation & 115.7 & 7.7 \\
\hline 71 & 04067800 & Armstrong Ck nr Armstrong Ck & $45^{\circ} 39^{\prime} 29^{\prime \prime}$ & $88^{\circ} 28^{\prime} 44^{\prime \prime}$ & 04063700 & 23.2 & .2300 & relation & 10.4 & 6.1 \\
\hline 72 & 04067840 & Peshtigo R nr Blackwell & $45^{\circ} 30^{\prime} 42^{\prime \prime}$ & $88^{\circ} 26^{\prime} 00^{\prime \prime}$ & 04066500 & 268 & .3631 & equation & 160.5 & 8.1 \\
\hline
\end{tabular}

${ }^{1}$ Low-flow discharge measurements at partial-record stations were compared to one or more nearby USGS gaging stations to establish a relation between measured discharge and concurrent daily discharge. The nearby gaging station that provided the best relation with the partial-record station was chosen as the index station and was associated with nearby miscellaneous measurement sites.

${ }^{2}$ Mismatch between surface-water and ground-water drainage area. 
Appendix 5D. Average annual base flow and corresponding recharge, 1970-99, for partial-record stations in the Menominee-0conto-Peshtigo River Basin-Continued.

[USGS, U.S. Geological Survey; ID, identification number; $\mathrm{dd}^{\circ} \mathrm{mm}$ 'ss", degrees, minutes, seconds; $\mathrm{mi}^{2}$, square mile; $\mathrm{ft}^{3} / \mathrm{s} / \mathrm{mi}^{2}$, cubic foot per second per square mile; $\mathrm{ft}^{3} / \mathrm{s}$, cubic foot per second; equation, statewide regression equation; relation, established relation line with index station; R, River; Bk, Brook; Br, Branch; Fk, Fork; Ck, Creek; Tr, Tributary; nr, near; N, North; S, South; E, East; W, West;

,-- not determined in historical low-flow reports. See references for full list of low-flow reports.]

\begin{tabular}{|c|c|c|c|c|c|c|c|c|c|c|}
\hline $\begin{array}{c}\text { Map } \\
\text { number }\end{array}$ & $\begin{array}{c}\text { USGS } \\
\text { station ID }\end{array}$ & Station name & $\begin{array}{c}\text { Latitude } \\
\text { (dd }{ }^{\circ} \mathrm{mm} \text { 'ss") }\end{array}$ & $\begin{array}{c}\text { Longitude } \\
\text { (ddºmm'ss") }\end{array}$ & $\begin{array}{l}\text { Index } \\
\text { station }^{1}\end{array}$ & $\begin{array}{c}\text { Drainage } \\
\text { area } \\
\left(\mathrm{mi}^{2}\right)\end{array}$ & $\begin{array}{l}\text { Base flow } \\
\text { factor } \\
\left(\mathrm{ft}^{3} / \mathrm{s} / \mathrm{mi}^{2}\right)\end{array}$ & $\begin{array}{c}\text { Base flow } \\
\text { method }\end{array}$ & $\begin{array}{c}\text { Base flow } \\
\qquad\left(\mathrm{ft}^{3} / \mathrm{s}\right)\end{array}$ & $\begin{array}{c}\text { Recharge } \\
\text { (inches) }\end{array}$ \\
\hline 73 & 04067882 & Rat $\mathrm{R}$ at Laona & $45^{\circ} 33^{\prime} 27^{\prime \prime}$ & $88^{\circ} 40^{\prime} 00^{\prime \prime}$ & 04066500 & 32 & 0.5500 & relation & 25.0 & 10.6 \\
\hline 74 & 04067890 & Rat R nr Laona & $45^{\circ} 31^{\prime} 59^{\prime \prime}$ & $88^{\circ} 39^{\prime} 00^{\prime \prime}$ & 04066500 & 42.8 & .6785 & equation & 34.2 & 10.9 \\
\hline 75 & 04067900 & Rat R nr Wabeno & $45^{\circ} 29^{\prime} 00^{\prime \prime}$ & $88^{\circ} 29^{\prime} 52^{\prime \prime}$ & 04066500 & 82.1 & .6410 & relation & 66.0 & 10.9 \\
\hline 76 & 04067920 & Rat R nr Wabeno & $45^{\circ} 26^{\prime} 04^{\prime \prime}$ & $88^{\circ} 21^{\prime} 00^{\prime \prime}$ & 04066500 & 105 & .6096 & equation & 80.8 & 10.5 \\
\hline 77 & 04067950 & Otter Ck nr Lkwood & $45^{\circ} 24^{\prime} 44^{\prime \prime}$ & $88^{\circ} 21^{\prime} 00^{\prime \prime}$ & 04066500 & 32.1 & .5937 & equation & 23.8 & 10.1 \\
\hline 78 & 04067990 & Eagle $\mathrm{Ck}$ nr athelstane & $45^{\circ} 24^{\prime} 04^{\prime \prime}$ & $88^{\circ} 11^{\prime} 10^{\prime \prime}$ & 04066500 & 37.5 & .2620 & relation & 22.0 & 8.0 \\
\hline 79 & 04068100 & N Fk Thunder R nr Lkwood & $45^{\circ} 19^{\prime} 31^{\prime \prime}$ & $88^{\circ} 19^{\prime} 40^{\prime \prime}$ & 04066500 & 17 & .4760 & relation & 11.5 & 9.2 \\
\hline 80 & 04068190 & S Fk Thunder R nr Mountain & $45^{\circ} 17^{\prime} 09^{\prime \prime}$ & $88^{\circ} 17^{\prime} 00^{\prime \prime}$ & 04066500 & 21 & .2882 & equation & 10.6 & 6.9 \\
\hline 81 & 04068200 & Thunder R nr Lkwood & $45^{\circ} 16^{\prime} 58^{\prime \prime}$ & $88^{\circ} 15^{\prime} 00^{\prime \prime}$ & 04066500 & 53.5 & .4689 & equation & 35.4 & 9.0 \\
\hline 82 & 04069200 & Middle Inlet at Middle Inlet & $45^{\circ} 17^{\prime} 47^{\prime \prime}$ & $87^{\circ} 58^{\prime} 37^{\prime \prime}$ & 04066500 & 32.6 & .6339 & equation & 25.0 & 10.4 \\
\hline 83 & 04069290 & Middle Inlet nr Middle Inlet & $45^{\circ} 17^{\prime} 38^{\prime \prime}$ & $87^{\circ} 56^{\prime} 16^{\prime \prime}$ & 04066500 & 59.2 & .4570 & relation & 33.0 & 7.6 \\
\hline 84 & 04069295 & Upper Inlet nr Mcallister & $45^{\circ} 17^{\prime} 29^{\prime \prime}$ & $87^{\circ} 50^{\prime} 01^{\prime \prime}$ & 04066500 & 7.35 & .1575 & equation & 2.7 & 4.9 \\
\hline 85 & 04069297 & Smith Ck nr Crivitz & $45^{\circ} 15^{\prime} 21^{\prime \prime}$ & $87^{\circ} 59^{\prime} 29^{\prime \prime}$ & 04066500 & 11.3 & -- & relation & 4.1 & 4.9 \\
\hline 86 & 04069350 & S Br Beaver Ck at Beaver & $45^{\circ} 07^{\prime} 50^{\prime \prime}$ & $88^{\circ} 01^{\prime} 02^{\prime \prime}$ & 04066500 & 51 & .3010 & relation & 25.0 & 6.7 \\
\hline 87 & 04069380 & Little Peshtigo R nr Colman & $45^{\circ} 03^{\prime} 23^{\prime \prime}$ & $88^{\circ} 05^{\prime} 00^{\prime \prime}$ & 04071000 & 21.3 & .3521 & equation & 11.9 & 7.6 \\
\hline 88 & 04069387 & Little Peshtigo $\mathrm{R}$ at Coleman & $45^{\circ} 04^{\prime} 39^{\prime \prime}$ & $88^{\circ} 02^{\prime} 00^{\prime \prime}$ & 04071000 & 34.3 & .2090 & relation & 12.2 & 4.8 \\
\hline 89 & 04069390 & Little Peshtigo R nr Coleman & $45^{\circ} 03^{\prime} 54^{\prime \prime}$ & $87^{\circ} 59^{\prime} 44^{\prime \prime}$ & 04071000 & 41.6 & .2180 & relation & 14.0 & 4.6 \\
\hline 90 & 04069480 & Trout Ck nr Peshtigo & $45^{\circ} 03^{\prime} 35^{\prime \prime}$ & $87^{\circ} 46^{\prime} 44^{\prime \prime}$ & 04071000 & 24.4 & .0309 & equation & 3.9 & 2.2 \\
\hline 91 & 04069690 & N Br Oconto R at Wabeno & $45^{\circ} 26^{\prime} 32^{\prime \prime}$ & $88^{\circ} 39^{\prime} 00^{\prime \prime}$ & 04071000 & 31.3 & -- & relation & 22.0 & 9.5 \\
\hline 92 & 04069800 & N Br Oconto R nr Carter & $45^{\circ} 23^{\prime} 50^{\prime \prime}$ & $88^{\circ} 34^{\prime} 00^{\prime \prime}$ & 04071000 & 58.2 & .3995 & equation & 35.5 & 8.3 \\
\hline 93 & 04069900 & N Br Oconto R nr Lkwood & $45^{\circ} 20^{\prime} 13^{\prime \prime}$ & $88^{\circ} 27^{\prime} 00^{\prime \prime}$ & 04071000 & 98.3 & .5558 & equation & 72.0 & 9.9 \\
\hline 94 & 04070063 & Mccaslin Bk nr Lkwood & $45^{\circ} 18^{\prime} 04^{\prime \prime}$ & $88^{\circ} 29^{\prime} 00^{\prime \prime}$ & 04071000 & 53.1 & .3200 & relation & 28.0 & 7.2 \\
\hline 95 & 04070100 & N Br Oconto R nr Mountain & $45^{\circ} 13^{\prime} 57^{\prime \prime}$ & $88^{\circ} 28^{\prime} 30^{\prime \prime}$ & 04071000 & 174 & .5240 & relation & 150.0 & 11.7 \\
\hline 96 & 04070300 & Waupee Ck nr Mountain & $45^{\circ} 07^{\prime} 52^{\prime \prime}$ & $88^{\circ} 26^{\prime} 01^{\prime \prime}$ & 04071000 & 51.4 & .3380 & relation & 26.5 & 7.0 \\
\hline
\end{tabular}

${ }^{1}$ Low-flow discharge measurements at partial-record stations were compared to one or more nearby USGS gaging stations to establish a relation between measured discharge and concurrent daily discharge. The nearby gaging station that provided the best relation with the partial-record station was chosen as the index station and was associated with nearby miscellaneous measurement sites.

${ }^{2}$ Mismatch between surface-water and ground-water drainage area. 
[USGS, U.S. Geological Survey; ID, identification number; $\mathrm{dd}^{\circ} \mathrm{mm}$ 'ss", degrees, minutes, seconds; $\mathrm{mi}^{2}$, square mile; $\mathrm{ft}^{3} / \mathrm{s} / \mathrm{mi}^{2}$, cubic foot per second per square mile; $\mathrm{ft}^{3} / \mathrm{s}$, cubic foot per second; equation, statewide regression equation; relation, established relation line with index station; R, River; Bk, Brook; Br, Branch; Fk, Fork; Ck, Creek; Tr, Tributary; nr, near; N, North; S, South; E, East; W, West; ,-- not determined in historical low-flow reports. See references for full list of low-flow reports.]

\begin{tabular}{|c|c|c|c|c|c|c|c|c|c|c|}
\hline $\begin{array}{c}\text { Map } \\
\text { number }\end{array}$ & $\begin{array}{c}\text { USGS } \\
\text { station ID }\end{array}$ & Station name & $\begin{array}{c}\text { Latitude } \\
\text { (dd }{ }^{\circ} \mathrm{mm} \text { 'ss") }\end{array}$ & $\begin{array}{c}\text { Longitude } \\
\text { (ddºmm'ss") }\end{array}$ & $\begin{array}{l}\text { Index } \\
\text { station }^{1}\end{array}$ & $\begin{array}{c}\text { Drainage } \\
\text { area } \\
\left(\mathrm{mi}^{2}\right)\end{array}$ & $\begin{array}{c}\text { Base flow } \\
\text { factor } \\
\left(\mathrm{ft}^{3} / \mathrm{s} / \mathrm{mi}^{2}\right)\end{array}$ & $\begin{array}{l}\text { Base flow } \\
\text { method }\end{array}$ & $\begin{array}{c}\text { Base flow } \\
\left(\mathrm{ft}^{3} / \mathrm{s}\right)\end{array}$ & $\begin{array}{c}\text { Recharge } \\
\text { (inches) }\end{array}$ \\
\hline 97 & 04070420 & S Br Oconto R nr Langlade & $45^{\circ} 11^{\prime} 34^{\prime \prime}$ & $88^{\circ} 40^{\prime} 00^{\prime \prime}$ & 04071000 & 24 & 0.5432 & equation & 16.9 & 9.6 \\
\hline 98 & 04070600 & Hills Pond Ck nr Langlade & $45^{\circ} 11^{\prime} 50^{\prime \prime}$ & $88^{\circ} 37^{\prime} 15^{\prime \prime}$ & 04071000 & 8.92 & .9280 & relation & 12.5 & ${ }^{2} 19.0$ \\
\hline 99 & 04070606 & S Br Oconto R nr Boulder Lk & $45^{\circ} 10^{\prime} 17^{\prime \prime}$ & $88^{\circ} 37^{\prime} 07^{\prime \prime}$ & 04071000 & 50.47 & .6024 & equation & 38.0 & 10.2 \\
\hline 100 & 04070680 & 1st S Br Oconto R nr Mountain & $45^{\circ} 12^{\prime} 14^{\prime \prime}$ & $88^{\circ} 31^{\prime} 00^{\prime \prime}$ & 04071000 & 20.2 & .5389 & equation & 14.1 & 9.5 \\
\hline 101 & 04070720 & S Br Oconto R nr Breed & $45^{\circ} 03^{\prime} 40^{\prime \prime}$ & $88^{\circ} 31^{\prime} 24^{\prime \prime}$ & 04071000 & 143 & .6156 & equation & 111.3 & 10.6 \\
\hline 102 & 04070800 & Pecore Ck nr Hayes & $45^{\circ} 00^{\prime} 28^{\prime \prime}$ & $88^{\circ} 26^{\prime} 55^{\prime \prime}$ & 04071000 & 31.2 & .3930 & relation & 21.0 & 9.1 \\
\hline 103 & 04070850 & Peshtigo Bk nr Suring & $45^{\circ} 06^{\prime} 48^{\prime \prime}$ & $88^{\circ} 16^{\prime} 00^{\prime \prime}$ & 04071000 & 60.5 & .0920 & equation & 17.2 & 3.9 \\
\hline 104 & 04070860 & Peshtigo Bk nr Suring & $45^{\circ} 02^{\prime} 31^{\prime \prime}$ & $88^{\circ} 20^{\prime} 00^{\prime \prime}$ & 04071000 & 109 & .1227 & equation & 36.5 & 4.5 \\
\hline 105 & 04070950 & Linzy Ck nr Underhill & $44^{\circ} 55^{\prime} 09^{\prime \prime}$ & $88^{\circ} 26^{\prime} 00^{\prime \prime}$ & 04071000 & 35.8 & .4699 & equation & 23.6 & 8.9 \\
\hline 106 & 04071090 & Christie Ck at Gillett & $44^{\circ} 53^{\prime} 38^{\prime \prime}$ & $88^{\circ} 18^{\prime} 00^{\prime \prime}$ & 04071000 & 7.27 & .1330 & relation & 2.7 & 5.0 \\
\hline 107 & 04071100 & Christie Ck nr Gillett & $44^{\circ} 52^{\prime} 43^{\prime \prime}$ & $88^{\circ} 16^{\prime} 00^{\prime \prime}$ & 04071000 & 12.7 & .1908 & equation & 5.1 & 5.5 \\
\hline 108 & 04071500 & Oconto $\mathrm{R}$ at Stiles & $44^{\circ} 51^{\prime} 31^{\prime \prime}$ & $88^{\circ} 03^{\prime} 00^{\prime \prime}$ & 04071000 & 771 & .3879 & equation & 488.1 & 8.6 \\
\hline 109 & 04071703 & Little R nr Lena & $45^{\circ} 00^{\prime} 09^{\prime \prime}$ & $88^{\circ} 01^{\prime} 00^{\prime \prime}$ & 04071000 & 23.2 & .0719 & equation & 5.7 & 3.3 \\
\hline 110 & 04071720 & Kelly Bk nr Oconto Falls & $44^{\circ} 57^{\prime} 05^{\prime \prime}$ & $88^{\circ} 09^{\prime} 00^{\prime \prime}$ & 04071000 & 57.9 & .0959 & equation & 16.8 & 3.9 \\
\hline 111 & 04071730 & Kelly Bk nr Lena & $44^{\circ} 58^{\prime} 22^{\prime \prime}$ & $88^{\circ} 12^{\prime} 45^{\prime \prime}$ & 04071000 & 80 & .0660 & relation & 15.0 & 2.5 \\
\hline 112 & 04071733 & Kelly Bk nr Lena & $44^{\circ} 59^{\prime} 24^{\prime \prime}$ & $88^{\circ} 01^{\prime} 00^{\prime \prime}$ & 04071000 & 81.44 & .0461 & equation & 16.3 & 2.7 \\
\hline 113 & 04071746 & Little $\mathrm{R}$ atLena, Little $\mathrm{R}$ Tr at Lena & $44^{\circ} 57^{\prime} 23^{\prime \prime}$ & $88^{\circ} 02^{\prime} 00^{\prime \prime}$ & 04071000 & 2.41 & .2027 & equation & 1.0 & 5.5 \\
\hline 114 & 04071760 & Little R nr Stiles & $44^{\circ} 53^{\prime} 19^{\prime \prime}$ & $88^{\circ} 00^{\prime} 00^{\prime \prime}$ & 04071000 & 170 & .0542 & equation & 37.5 & 3.0 \\
\hline 115 & 04071788 & Pensaukee R nr Zachow & $44^{\circ} 43^{\prime} 09^{\prime \prime}$ & $88^{\circ} 19^{\prime} 00^{\prime \prime}$ & 04071000 & 19.3 & .1000 & relation & 2.5 & 1.8 \\
\hline 116 & 04071795 & Pensaukee R nr Krakow & $44^{\circ} 45^{\prime} 09^{\prime \prime}$ & $88^{\circ} 16^{\prime} 35^{\prime \prime}$ & 04071000 & 35.8 & .0167 & equation & 4.1 & 1.6 \\
\hline 117 & 04071800 & Pensaukee R nr Pulaski & $44^{\circ} 45^{\prime} 48^{\prime \prime}$ & $88^{\circ} 15^{\prime} 07^{\prime \prime}$ & 04071000 & 48.8 & -- & relation & 6.0 & 1.7 \\
\hline 118 & 04071843 & N Br Pensaukee R nr Sampson & $44^{\circ} 47^{\prime} 36^{\prime \prime}$ & $88^{\circ} 09^{\prime} 18^{\prime \prime}$ & 04071000 & 39.9 & .0453 & equation & 7.8 & 2.7 \\
\hline 119 & 04071850 & Pensaukee R nr Abrams & $44^{\circ} 47^{\prime} 28^{\prime \prime}$ & $88^{\circ} 02^{\prime} 56^{\prime \prime}$ & 04071000 & 116 & .0600 & relation & 27.0 & 3.2 \\
\hline 120 & 04071888 & Little Suamico R nr Pulaski & $44^{\circ} 41^{\prime} 03^{\prime \prime}$ & $88^{\circ} 12^{\prime} 58^{\prime \prime}$ & 04071000 & 9.73 & -- & relation & .7 & 1.0 \\
\hline
\end{tabular}

${ }^{1}$ Low-flow discharge measurements at partial-record stations were compared to one or more nearby USGS gaging stations to establish a relation between measured discharge and concurrent daily discharge. The nearby gaging station that provided the best relation with the partial-record station was chosen as the index station and was associated with nearby miscellaneous measurement sites.

${ }^{2}$ Mismatch between surface-water and ground-water drainage area. 
Appendix 5D. Average annual base flow and corresponding recharge, 1970-99, for partial-record stations in the Menominee-0conto-Peshtigo River Basin-Continued.

[USGS, U.S. Geological Survey; ID, identification number; $\mathrm{dd}^{\circ} \mathrm{mm}$ 'ss", degrees, minutes, seconds; $\mathrm{mi}^{2}$, square mile; $\mathrm{ft}^{3} / \mathrm{s} / \mathrm{mi}^{2}$, cubic foot per second per square mile; $\mathrm{ft}^{3} / \mathrm{s}$, cubic foot per second; equation, statewide regression equation; relation, established relation line with index station; R, River; Bk, Brook; Br, Branch; Fk, Fork; Ck, Creek; Tr, Tributary; nr, near; N, North; S, South; E, East; W, West; ,-- not determined in historical low-flow reports. See references for full list of low-flow reports.]

\begin{tabular}{|c|c|c|c|c|c|c|c|c|c|c|}
\hline $\begin{array}{c}\text { Map } \\
\text { number }\end{array}$ & $\begin{array}{c}\text { USGS } \\
\text { station ID }\end{array}$ & Station name & $\begin{array}{c}\text { Latitude } \\
\text { (dd }{ }^{\circ} \mathrm{mm} \text { 'ss") }\end{array}$ & $\begin{array}{c}\text { Longitude } \\
\text { (ddºmm'ss") }\end{array}$ & $\begin{array}{l}\text { Index } \\
\text { station }{ }^{1}\end{array}$ & $\begin{array}{c}\text { Drainage } \\
\text { area } \\
\left(\mathrm{mi}^{2}\right)\end{array}$ & $\begin{array}{c}\text { Base flow } \\
\text { factor } \\
\left(\mathrm{ft}^{3} / \mathrm{s} / \mathrm{mi}^{2}\right)\end{array}$ & $\begin{array}{l}\text { Base flow } \\
\text { method }\end{array}$ & $\begin{array}{c}\text { Base flow } \\
\left(\mathrm{ft}^{3} / \mathrm{s}\right)\end{array}$ & $\begin{array}{c}\text { Recharge } \\
\text { (inches) }\end{array}$ \\
\hline 121 & 04071894 & Little Suamico R Tr nr Pulaski & $44^{\circ} 41^{\prime} 17^{\prime \prime}$ & $88^{\circ} 11^{\prime} 06^{\prime \prime}$ & 04071000 & 9.05 & 0.0126 & equation & 0.9 & 1.3 \\
\hline 122 & 04071899 & Little Suamico R Tr nr Pulaski & $44^{\circ} 40^{\prime} 57^{\prime \prime}$ & $88^{\circ} 11^{\prime} 02^{\prime \prime}$ & 04071000 & 6.87 & .0180 & equation & .8 & 1.6 \\
\hline 123 & 04071906 & Little Suamico R nr Sobieski & $44^{\circ} 42^{\prime} 34^{\prime \prime}$ & $88^{\circ} 04^{\prime} 09^{\prime \prime}$ & 04071000 & 45.9 & -- & relation & 4.4 & 1.3 \\
\hline 124 & 04071915 & Little Suamico R at Little Suamico & $44^{\circ} 42^{\prime} 38^{\prime \prime}$ & $88^{\circ} 00^{\prime} 48^{\prime \prime}$ & 04071000 & 59.5 & .0165 & equation & 6.9 & 1.6 \\
\hline 125 & 04072000 & Suamico $\mathrm{R}$ at Suamico & $44^{\circ} 37^{\prime} 55^{\prime \prime}$ & $88^{\circ} 03^{\prime} 09^{\prime \prime}$ & 04071000 & 55.64 & .0440 & relation & 6.2 & 1.5 \\
\hline 126 & 04072036 & Duck Ck nr Freedom & $44^{\circ} 25^{\prime} 55^{\prime \prime}$ & $88^{\circ} 14^{\prime} 45^{\prime \prime}$ & 04071000 & 24 & .0050 & equation & 1.5 & .8 \\
\hline 127 & 04072041 & Duck Ck Tr nr Oneida & $44^{\circ} 26^{\prime} 40^{\prime \prime}$ & $88^{\circ} 13^{\prime} 53^{\prime \prime}$ & 04071000 & 17.1 & .0122 & equation & 1.7 & 1.3 \\
\hline 128 & 04072050 & Duck Ck nr Oneida & $44^{\circ} 27^{\prime} 57^{\prime \prime}$ & $88^{\circ} 13^{\prime} 08^{\prime \prime}$ & 04071000 & 95.5 & .0030 & relation & 1.6 & .2 \\
\hline 129 & 04072175 & Trout Ck nr Oneida & $44^{\circ} 31^{\prime} 24^{\prime \prime}$ & $88^{\circ} 08^{\prime} 48^{\prime \prime}$ & 04071000 & 14.9 & .0687 & equation & 3.5 & 3.2 \\
\hline 130 & 04084426 & Mud Ck at Appleton & $44^{\circ} 20^{\prime} 53^{\prime \prime}$ & $88^{\circ} 23^{\prime} 00^{\prime \prime}$ & 04071000 & 10.4 & .0453 & equation & 2.0 & 2.6 \\
\hline
\end{tabular}

${ }^{1}$ Low-flow discharge measurements at partial-record stations were compared to one or more nearby USGS gaging stations to establish a relation between measured discharge and concurrent daily discharge. The nearby gaging station that provided the best relation with the partial-record station was chosen as the index station and was associated with nearby miscellaneous measurement sites.

${ }^{2}$ Mismatch between surface-water and ground-water drainage area. 


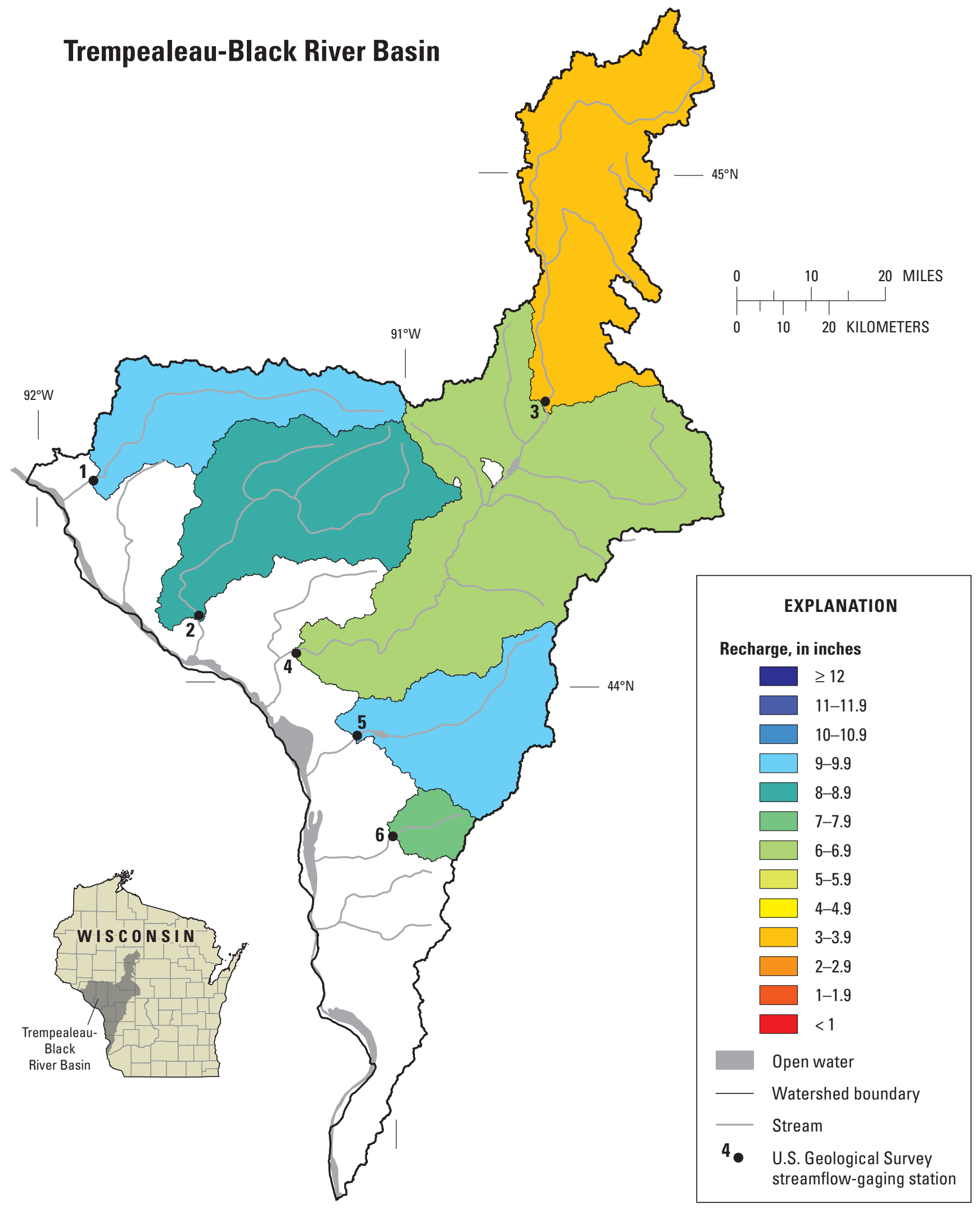

Appendix 6-A. Locations of streamflow-gaging stations in the Trempealeau-Black River Basin and spatial distribution of average annual recharge. 


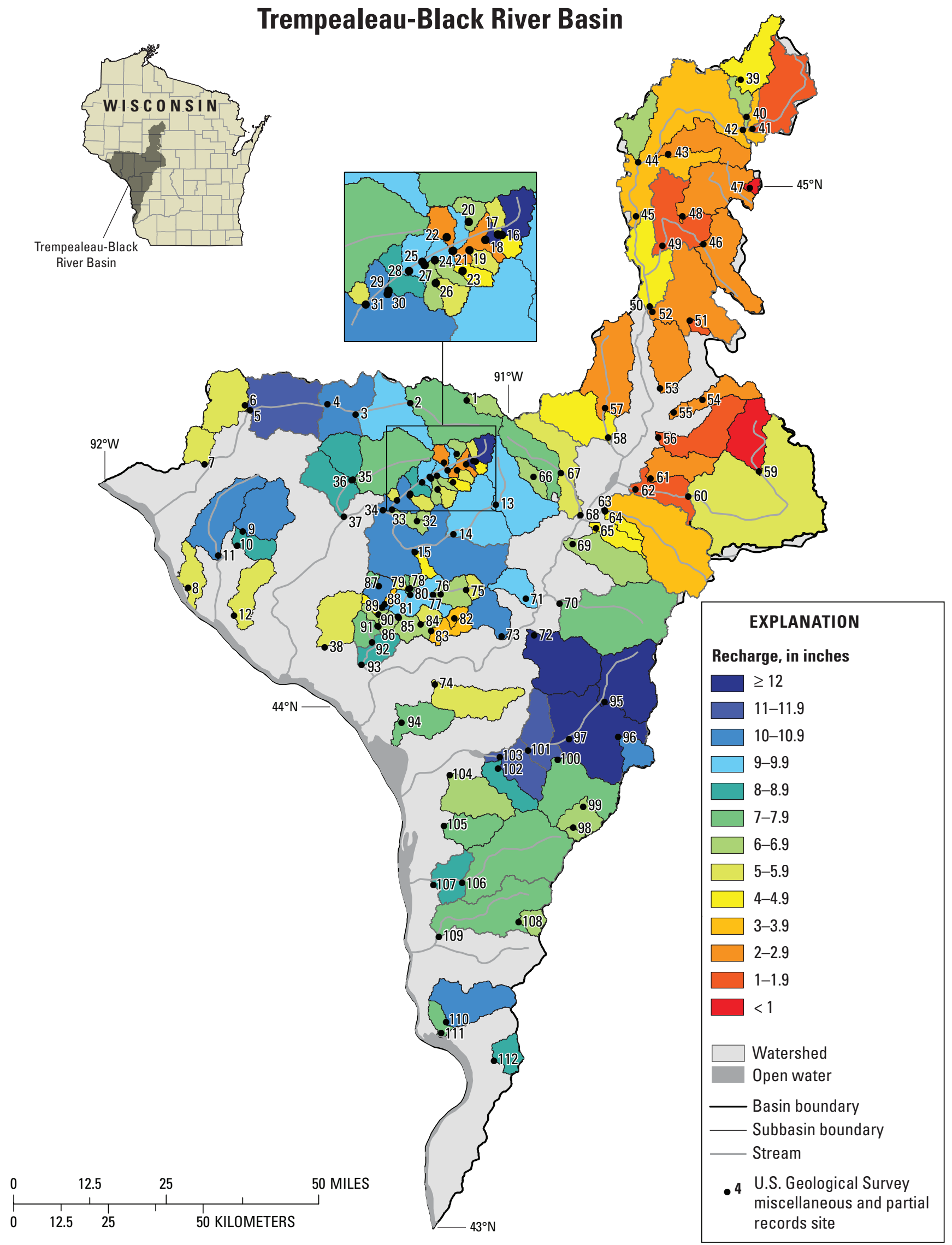

Appendix 6-B. Locations of miscellaneous and partial-record sites in the Trempealeau-Black River Basin and spatial distribution of estimated average annual recharge. 
[USGS, U.S. Geological Survey; ID, identification number; mi² $^{2}$ square mile; $\mathrm{ft}^{3}$ /s, cubic foot per second; BFI, Baseflow Index; ex rec, extended record; R, River; Bk, Brook; Br, Branch; Fk, Fork; Ck, Creek; Tr, Tributary; nr, near; N, North; S, South; E, East; W, West; --, not determined in historical low-flow reports. See references for full list of low-flow reports.]

\begin{tabular}{|c|c|c|c|c|c|c|c|c|c|c|c|}
\hline $\begin{array}{c}\text { Map } \\
\text { number }\end{array}$ & $\begin{array}{l}\text { USGS } \\
\text { station } \\
\text { ID }\end{array}$ & Station name & $\begin{array}{c}\text { Drainage } \\
\text { area } \\
\left(\mathrm{mi}^{2}\right)\end{array}$ & $\begin{array}{c}\text { Period of } \\
\text { analysis } \\
\text { (water years }{ }^{1} \text { ) }\end{array}$ & $\begin{array}{c}\text { Base flow } \\
\text { method }\end{array}$ & $\begin{array}{l}\text { Base flow } \\
\text { index }\end{array}$ & $\begin{array}{l}\text { Base } \\
\text { flow } \\
\left(\mathrm{ft}^{3} / \mathrm{s}\right)\end{array}$ & $\begin{array}{c}\text { Recharge } \\
\text { (inches) }\end{array}$ & $\begin{array}{c}\text { Total } \\
\text { streamflow } \\
\left(\mathrm{ft}^{3} / \mathbf{s}\right)\end{array}$ & $\begin{array}{c}\text { Total } \\
\text { streamflow } \\
\text { (inches) }\end{array}$ & $\begin{array}{r}{ }^{2} 090 \\
\left(\mathrm{ft}^{3} / \mathrm{s}\right)\end{array}$ \\
\hline 1 & 05372000 & Buffalo R nr Tell & 406 & 1970-1999 & ex rec & -- & 270 & 9.0 & -- & -- & 140 \\
\hline 2 & 05379500 & Trempealeau R at Dodge & 643 & 1970-1999 & BFI & 0.7590 & 400 & 8.5 & 530 & 11.3 & 200 \\
\hline 3 & 05381000 & Black R at Neillsville & 749 & 1970-1999 & BFI & .3130 & 200 & 3.7 & 660 & 12.0 & 54 \\
\hline 4 & 05382000 & Black R nr Galesville & 2080 & 1970-1999 & BFI & .5390 & 1,000 & 6.7 & 2,000 & 12.8 & 510 \\
\hline 5 & 05383000 & La Crosse R nr W Salem & 396 & $1915-1970$ & ex rec & -- & 280 & 9.7 & -- & -- & 180 \\
\hline 6 & 05386500 & Coon Ck at Coon Valley & 77.2 & $\begin{array}{c}1936-1940 \\
1979-1981\end{array}$ & ex rec & -- & 45 & 7.9 & -- & -- & 31 \\
\hline
\end{tabular}

${ }^{1}$ Water year is a 12-month period from October 1 through September 30, and is designated by the calendar year in which it ends (for example, the 2004 water year occurred October 1,2003 through September 30, 2004).

${ }^{2}$ Discharge that is exceeded 90 percent of the time. 
Appendix 6D. Average annual base flow and corresponding recharge, 1970-99, for partial-record stations in the Trempealeau-Black River Basin.

[USGS, U.S. Geological Survey; ID, identification number; $\mathrm{dd}^{\circ} \mathrm{mm}$ 'ss", degrees, minutes, seconds; $\mathrm{mi}^{2}$, square mile; $\mathrm{ft}^{3} / \mathrm{s} / \mathrm{mi}^{2}$, cubic foot per second per square mile; $\mathrm{ft}^{3} / \mathrm{s}$, cubic foot per second; equation, statewide regression equation; relation, established relation line with index station; R, River; Bk, Brook; Br, Branch; Fk, Fork; Ck, Creek; Tr, Tributary; nr, near; N, North; S, South; E, East; W, West;

--, not determined in historical low-flow reports. See references for full list of low-flow reports.]

\begin{tabular}{|c|c|c|c|c|c|c|c|c|c|c|}
\hline $\begin{array}{c}\text { Map } \\
\text { number }\end{array}$ & $\begin{array}{c}\text { USGS } \\
\text { station ID }\end{array}$ & Station name & $\begin{array}{c}\text { Latitude } \\
\text { (dd }{ }^{\circ} \mathrm{mm} \text { 'ss") }\end{array}$ & $\begin{array}{c}\text { Longitude } \\
\text { (dd }{ }^{\circ} \mathrm{mm} \text { 'ss") }\end{array}$ & $\begin{array}{l}\text { Index } \\
\text { station }{ }^{1}\end{array}$ & $\begin{array}{c}\text { Drainage } \\
\text { area } \\
\left(\mathrm{mi}^{2}\right)\end{array}$ & $\begin{array}{c}\text { Base flow } \\
\text { factor } \\
\left(\mathrm{ft}^{3} / \mathrm{s} / \mathrm{mi}^{2}\right)\end{array}$ & $\begin{array}{l}\text { Base flow } \\
\text { method }\end{array}$ & $\begin{array}{c}\text { Base flow } \\
\quad\left(\mathrm{ft}^{3} / \mathrm{s}\right)\end{array}$ & $\begin{array}{c}\text { Recharge } \\
\text { (inches) }\end{array}$ \\
\hline 1 & 05371798 & N Fk Buffalo R nr Osseo & $44^{\circ} 35^{\prime} 07^{\prime \prime}$ & $91^{\circ} 05^{\prime} 40^{\prime \prime}$ & 05379500 & 8.93 & 0.2748 & equation & 4.3 & 6.6 \\
\hline 2 & 05371830 & Buffalo R at Osseo & $44^{\circ} 34^{\prime} 41^{\prime \prime}$ & $91^{\circ} 14^{\prime} 48^{\prime \prime}$ & 05379500 & 72.8 & -- & relation & 41.9 & 7.8 \\
\hline 3 & 05371850 & Buffalo R at Strum & $44^{\circ} 33^{\prime} 16^{\prime \prime}$ & $91^{\circ} 23^{\prime} 36^{\prime \prime}$ & 05379500 & 123 & -- & relation & 90.2 & 10.0 \\
\hline 4 & 05371860 & Buffalo R at Eleva & $44^{\circ} 34^{\prime} 26^{\prime \prime}$ & $91^{\circ} 28^{\prime} 09^{\prime \prime}$ & 05379500 & 165 & -- & relation & 121.8 & 10.0 \\
\hline 5 & 05371895 & Buffalo R at Mondovi & $44^{\circ} 33^{\prime} 35^{\prime \prime}$ & $91^{\circ} 40^{\prime} 38^{\prime \prime}$ & 05379500 & 238 & -- & relation & 184.9 & 10.6 \\
\hline 6 & 05371910 & Harvey $\mathrm{Ck}$ at Mondovi & $44^{\circ} 34^{\prime} 06^{\prime \prime}$ & $91^{\circ} 41^{\prime} 27^{\prime \prime}$ & 05379500 & 36.9 & .2000 & equation & 15.6 & 5.7 \\
\hline 7 & 05371960 & Tamarack Ck at Mondovi & $44^{\circ} 27^{\prime} 14^{\prime \prime}$ & $91^{\circ} 47^{\prime} 48^{\prime \prime}$ & 05379500 & 15.4 & .2149 & equation & 6.6 & 5.8 \\
\hline 8 & 05377700 & Cochrane Ditch at Cochrane & $44^{\circ} 12^{\prime} 57^{\prime \prime}$ & $91^{\circ} 49^{\prime} 59^{\prime \prime}$ & 05379500 & 17.7 & -- & relation & 7.3 & 5.6 \\
\hline 9 & 05378050 & Waumandee Ck nr Waumandee & $44^{\circ} 19^{\prime} 34^{\prime \prime}$ & $91^{\circ} 41^{\prime} 22^{\prime \prime}$ & 05379500 & 41.6 & -- & relation & 32.7 & 10.7 \\
\hline 10 & 05378060 & Waumandee $\mathrm{Ck}$ at Waumandee & $44^{\circ} 17^{\prime} 54^{\prime \prime}$ & $91^{\circ} 42^{\prime} 14^{\prime \prime}$ & 05379500 & 56.1 & .4613 & equation & 36.9 & 8.9 \\
\hline 11 & 05378100 & Little Waumandee Ck nr Cream & $44^{\circ} 16^{\prime} 42^{\prime \prime}$ & $91^{\circ} 45^{\prime} 17^{\prime \prime}$ & 05379500 & 46.2 & .3940 & relation & 35.3 & 10.4 \\
\hline 12 & 05378200 & Eagle Ck nr Fountain City & $44^{\circ} 09^{\prime} 49^{\prime \prime}$ & $91^{\circ} 42^{\prime} 28^{\prime \prime}$ & 05379500 & 26.6 & .3500 & relation & 11.0 & 5.6 \\
\hline 13 & 05379180 & Trempealeau R at Hixton & $44^{\circ} 23^{\prime} 07^{\prime \prime}$ & $91^{\circ} 00^{\prime} 48^{\prime \prime}$ & 05379500 & 57.6 & -- & relation & 39.2 & 9.2 \\
\hline 14 & 05379200 & Trempealeau R at Taylor & $44^{\circ} 19^{\prime} 38^{\prime \prime}$ & $91^{\circ} 07 ' 30^{\prime \prime}$ & 05379500 & 110 & .3720 & relation & 74.0 & 9.1 \\
\hline 15 & 05379212 & Tappen Coulee at Blair & $44^{\circ} 17^{\prime} 31^{\prime \prime}$ & $91^{\circ} 13^{\prime} 42^{\prime \prime}$ & 05379500 & 4.48 & -- & relation & 1.5 & 4.5 \\
\hline 16 & 05379220 & Lyons Ck nr Nfield & $44^{\circ} 28^{\prime} 06^{\prime \prime}$ & $91^{\circ} 04^{\prime} 07^{\prime \prime}$ & 05379500 & 2.18 & .1444 & equation & .7 & 4.6 \\
\hline 17 & 05379224 & Ellison Coulee nr Nfield & $44^{\circ} 28^{\prime} 07^{\prime \prime}$ & $91^{\circ} 04^{\prime} 29^{\prime \prime}$ & 05379500 & 2.49 & .2140 & equation & 1.0 & 5.6 \\
\hline 18 & 05379226 & Pigeon $\mathrm{Ck}$ at Nfield & $44^{\circ} 27^{\prime} 44^{\prime \prime}$ & $91^{\circ} 05^{\prime} 40^{\prime \prime}$ & 05379500 & 13.9 & .5977 & equation & 10.2 & 9.9 \\
\hline 19 & 05379228 & May Coulee nr York & $44^{\circ} 27^{\prime} 02^{\prime \prime}$ & $91^{\circ} 07^{\prime} 05^{\prime \prime}$ & 05379500 & 2.85 & .1990 & equation & 1.1 & 5.4 \\
\hline 20 & 05379230 & Beaver Ck nr Nfield & $44^{\circ} 28^{\prime} 55^{\prime \prime}$ & $91^{\circ} 07^{\prime} 13^{\prime \prime}$ & 05379500 & 3.38 & .3036 & equation & 1.7 & 6.8 \\
\hline 21 & 05379232 & Beaver $\mathrm{Ck}$ at York & $44^{\circ} 26^{\prime} 59^{\prime \prime}$ & $91^{\circ} 08^{\prime} 39^{\prime \prime}$ & 05379500 & 6.58 & .3702 & equation & 3.7 & 7.6 \\
\hline 22 & 05379234 & Pigeon Ck at York & $44^{\circ} 27^{\prime} 52^{\prime \prime}$ & $91^{\circ} 09^{\prime} 13^{\prime \prime}$ & 05379500 & 33.7 & .4059 & equation & 20.5 & 8.3 \\
\hline 23 & 05379238 & Schermerhorn Ck nr York & $44^{\circ} 25^{\prime} 39^{\prime \prime}$ & $91^{\circ} 07^{\prime} 45^{\prime \prime}$ & 05379500 & 3.06 & .1281 & equation & 1.0 & 4.3 \\
\hline 24 & 05379240 & Schermerhorn Ck nr York & $44^{\circ} 26^{\prime} 20^{\prime \prime}$ & $91^{\circ} 10^{\prime} 17^{\prime \prime}$ & 05379500 & 6.06 & .2010 & equation & 2.5 & 5.5 \\
\hline 25 & 05379242 & Pigeon Ck nr Pigeon Falls & $44^{\circ} 26^{\prime} 12^{\prime \prime}$ & $91^{\circ} 11^{\prime} 27^{\prime \prime}$ & 05379500 & 43.5 & .4782 & equation & 29.0 & 9.1 \\
\hline
\end{tabular}

${ }^{1}$ Low-flow discharge measurements at partial-record stations were compared to one or more nearby USGS gaging stations to establish a relation between measured discharge and concurrent daily discharge. The nearby gaging station that provided the best relation with the partial-record station was chosen as the index station and was associated with nearby miscellaneous measurement sites. 
Appendix 6D. Average annual base flow and corresponding recharge, 1970-99, for partial-record stations in the Trempealeau-Black River Basin—Continued.

[USGS, U.S. Geological Survey; ID, identification number; $\mathrm{dd}^{\circ} \mathrm{mm}$ 'ss", degrees, minutes, seconds; $\mathrm{mi}^{2}$, square mile; $\mathrm{ft}^{3} / \mathrm{s} / \mathrm{mi}^{2}$, cubic foot per second per square mile; $\mathrm{ft}^{3} / \mathrm{s}$, cubic foot per second; equation, statewide regression equation; relation, established relation line with index station; R, River; Bk, Brook; Br, Branch; Fk, Fork; Ck, Creek; Tr, Tributary; nr, near; N, North; S, South; E, East; W, West; ,-- not determined in historical low-flow reports. See references for full list of low-flow reports.]

\begin{tabular}{|c|c|c|c|c|c|c|c|c|c|c|}
\hline $\begin{array}{c}\text { Map } \\
\text { number }\end{array}$ & $\begin{array}{c}\text { USGS } \\
\text { station ID }\end{array}$ & Station name & $\begin{array}{c}\text { Latitude } \\
\text { (dd }{ }^{\circ} \mathrm{mm} \text { 'ss") }\end{array}$ & $\begin{array}{c}\text { Longitude } \\
\text { (dd }{ }^{\circ} \mathrm{mm} \text { 'ss") }\end{array}$ & $\begin{array}{c}\text { Index } \\
\text { station }^{1}\end{array}$ & $\begin{array}{c}\text { Drainage } \\
\text { area } \\
\left(\mathrm{mi}^{2}\right)\end{array}$ & $\begin{array}{c}\text { Base flow } \\
\text { factor } \\
\left(\mathrm{ft}^{3} / \mathrm{s} / \mathrm{mi}^{2}\right)\end{array}$ & $\begin{array}{l}\text { Base flow } \\
\text { method }\end{array}$ & $\begin{array}{c}\text { Base flow } \\
\left(\mathrm{ft}^{3} / \mathrm{s}\right)\end{array}$ & $\begin{array}{c}\text { Recharge } \\
\text { (inches) }\end{array}$ \\
\hline 26 & 05379244 & Big Slough nr Pigeon Falls & $44^{\circ} 24^{\prime} 49^{\prime \prime}$ & $91^{\circ} 10^{\prime} 11^{\prime \prime}$ & 05379500 & 4.67 & 0.1752 & equation & 1.8 & 5.1 \\
\hline 27 & 05379248 & Big Slough nr Pigeon Falls & $44^{\circ} 26^{\prime} 02^{\prime \prime}$ & $91^{\circ} 11^{\prime} 16^{\prime \prime}$ & 05379500 & 8.53 & .2031 & equation & 3.5 & 5.6 \\
\hline 28 & 05379249 & Pigeon Ck at Pigeon Falls & $44^{\circ} 25^{\prime} 36^{\prime \prime}$ & $91^{\circ} 12^{\prime} 41^{\prime \prime}$ & 05379500 & 58.4 & -- & relation & 42.5 & 9.9 \\
\hline 29 & 05379254 & Pigeon Ck nr Pigeon Falls & $44^{\circ} 24^{\prime} 15^{\prime \prime}$ & $91^{\circ} 14 ' 33^{\prime \prime}$ & 05379500 & 65.6 & .4432 & equation & 42.4 & 8.8 \\
\hline 30 & 05379260 & Fitch Coulee nr Pigeon Falls & $44^{\circ} 24^{\prime} 03^{\prime \prime}$ & $91^{\circ} 14^{\prime} 37^{\prime \prime}$ & 05379500 & 5.11 & .2266 & equation & 2.2 & 5.9 \\
\hline 31 & 05379263 & Thompson Coulee nr Whitehall & $44^{\circ} 23^{\prime} 14^{\prime \prime}$ & $91^{\circ} 16^{\prime} 33^{\prime \prime}$ & 05379500 & 2.06 & .2239 & equation & .9 & 5.7 \\
\hline 32 & 05379268 & Fly Ck nr Blair & $44^{\circ} 21^{\prime} 07^{\prime \prime}$ & $91^{\circ} 13^{\prime} 24^{\prime \prime}$ & 05379500 & 2.39 & .2513 & equation & 1.1 & 6.1 \\
\hline 33 & 05379275 & Fly Ck nr Whitehall & $44^{\circ} 22^{\prime} 23^{\prime \prime}$ & $91^{\circ} 17^{\prime} 29^{\prime \prime}$ & 05379500 & 9.54 & .2372 & equation & 4.3 & 6.1 \\
\hline 34 & 05379276 & Trempealeau R at Whitehall & $44^{\circ} 22^{\prime} 19^{\prime \prime}$ & $91^{\circ} 18^{\prime} 57^{\prime \prime}$ & 05379500 & 323 & -- & relation & 219.0 & 9.2 \\
\hline 35 & 05379296 & Elk Ck nr Independence & $44^{\circ} 25^{\prime} 46^{\prime \prime}$ & $91^{\circ} 23^{\prime} 49^{\prime \prime}$ & 05379500 & 56.2 & .3174 & equation & 30.4 & 7.4 \\
\hline 36 & 05379300 & Elk Ck at Elk Ck & $44^{\circ} 25^{\prime} 43^{\prime \prime}$ & $91^{\circ} 24^{\prime} 02^{\prime \prime}$ & 05379500 & 80.2 & .2190 & relation & 50.0 & 8.5 \\
\hline 37 & 05379320 & Elk Ck at Independence & $44^{\circ} 21^{\prime} 30^{\prime \prime}$ & $91^{\circ} 25^{\prime} 11^{\prime \prime}$ & 05379500 & 113 & -- & relation & 67.0 & 8.1 \\
\hline 38 & 05379550 & Tamarack Ck nr Galesville & $44^{\circ} 06^{\prime} 25^{\prime \prime}$ & $91^{\circ} 27^{\prime} 54^{\prime \prime}$ & 05379500 & 34.6 & .1682 & equation & 13.3 & 5.2 \\
\hline 39 & 05380801 & Black R nr Whittlesey & $45^{\circ} 12^{\prime} 21^{\prime \prime}$ & $90^{\circ} 21^{\prime} 43^{\prime \prime}$ & 05381000 & 34.8 & .1034 & equation & 10.4 & 4.1 \\
\hline 40 & 05380807 & Black R at Medford & $45^{\circ} 08^{\prime} 04^{\prime \prime}$ & $90^{\circ} 20^{\prime} 41^{\prime \prime}$ & 05381000 & 48.1 & -- & relation & 21.5 & 6.1 \\
\hline 41 & 05380820 & Little Black R nr Medford & $45^{\circ} 06^{\prime} 41^{\prime \prime}$ & $90^{\circ} 19^{\prime} 45^{\prime \prime}$ & 05381000 & 54.7 & .0170 & equation & 6.9 & 1.7 \\
\hline 42 & 05380825 & Black R nr Medford & $45^{\circ} 06^{\prime} 34^{\prime \prime}$ & $90^{\circ} 21^{\prime} 17^{\prime \prime}$ & 05381000 & 114 & .0833 & equation & 31.2 & 3.7 \\
\hline 43 & 05380870 & Pine Ck nr Medford & $45^{\circ} 03^{\prime} 44^{\prime \prime}$ & $90^{\circ} 33^{\prime} 24^{\prime \prime}$ & 05381000 & 36.7 & .0230 & relation & 6.2 & 2.3 \\
\hline 44 & 05380880 & Mckenzie Ck nr Lublin & $45^{\circ} 02^{\prime} 45^{\prime \prime}$ & $90^{\circ} 38^{\prime} 20^{\prime \prime}$ & 05381000 & 22.4 & -- & relation & 10.3 & 6.2 \\
\hline 45 & 05380886 & Black R nr Withee & $44^{\circ} 56^{\prime} 33^{\prime \prime}$ & $90^{\circ} 38^{\prime} 35^{\prime \prime}$ & 05381000 & 324 & .0810 & equation & 89.3 & 3.7 \\
\hline 46 & 05380889 & S Fk Poplar R nr Riplingler & $44^{\circ} 53^{\prime} 23^{\prime \prime}$ & $90^{\circ} 27^{\prime} 37^{\prime \prime}$ & 05381000 & 49.1 & .0266 & equation & 7.3 & 2.0 \\
\hline 47 & 05380891 & N Fk Poplar R at Dorchester & $44^{\circ} 59^{\prime} 55^{\prime \prime}$ & $90^{\circ} 20^{\prime} 08^{\prime \prime}$ & 05381000 & 2.41 & -- & relation & .1 & .4 \\
\hline 48 & 05380895 & N Fk Poplar R nr Owen & $44^{\circ} 56^{\prime} 37^{\prime \prime}$ & $90^{\circ} 31^{\prime} 04^{\prime \prime}$ & 05381000 & 52.6 & -- & relation & 9.0 & 2.3 \\
\hline 49 & 05380900 & Poplar R nr Owen & $44^{\circ} 53^{\prime} 10^{\prime \prime}$ & $90^{\circ} 34^{\prime} 17^{\prime \prime}$ & 05381000 & 155 & .0460 & relation & 21.0 & 1.8 \\
\hline 50 & 05380915 & Black R at Greenwood & $44^{\circ} 46^{\prime} 12^{\prime \prime}$ & $90^{\circ} 36^{\prime} 18^{\prime \prime}$ & 05381000 & 527 & -- & relation & 177.0 & 4.6 \\
\hline 51 & 05380945 & Bear Ck at Loyal & $44^{\circ} 44^{\prime} 32^{\prime \prime}$ & $90^{\circ} 29^{\prime} 45^{\prime \prime}$ & 05381000 & 3.32 & -- & relation & .4 & 1.6 \\
\hline
\end{tabular}

${ }^{1}$ Low-flow discharge measurements at partial-record stations were compared to one or more nearby USGS gaging stations to establish a relation between measured discharge and concurrent daily discharge. The nearby gaging station that provided the best relation with the partial-record station was chosen as the index station and was associated with nearby miscellaneous measurement sites. 
Appendix 6D. Average annual base flow and corresponding recharge, 1970-99, for partial-record stations in the Trempealeau-Black River Basin—Continued.

[USGS, U.S. Geological Survey; ID, identification number; $\mathrm{dd}^{\circ} \mathrm{mm}$ 'ss", degrees, minutes, seconds; $\mathrm{mi}^{2}$, square mile; $\mathrm{ft}^{3} / \mathrm{s} / \mathrm{mi}^{2}$, cubic foot per second per square mile; $\mathrm{ft}^{3} / \mathrm{s}$, cubic foot per second; equation, statewide regression equation; relation, established relation line with index station; R, River; Bk, Brook; Br, Branch; Fk, Fork; Ck, Creek; Tr, Tributary; nr, near; N, North; S, South; E, East; W, West; ,-- not determined in historical low-flow reports. See references for full list of low-flow reports.]

\begin{tabular}{|c|c|c|c|c|c|c|c|c|c|c|}
\hline $\begin{array}{c}\text { Map } \\
\text { number }\end{array}$ & $\begin{array}{c}\text { USGS } \\
\text { station ID }\end{array}$ & Station name & $\begin{array}{c}\text { Latitude } \\
\text { (dd }{ }^{\circ} \mathrm{mm} \text { 'ss") }\end{array}$ & $\begin{array}{l}\text { Longitude } \\
\text { (ddºmm'ss") }\end{array}$ & $\begin{array}{l}\text { Index } \\
\text { station }^{1}\end{array}$ & $\begin{array}{c}\text { Drainage } \\
\text { area } \\
\left(\mathrm{mi}^{2}\right)\end{array}$ & $\begin{array}{c}\text { Base flow } \\
\text { factor } \\
\left(\mathrm{ft}^{3} / \mathrm{s} / \mathrm{mi}^{2}\right)\end{array}$ & $\begin{array}{l}\text { Base flow } \\
\text { method }\end{array}$ & $\begin{array}{c}\text { Base flow } \\
\left(\mathrm{ft}^{3} / \mathrm{s}\right)\end{array}$ & $\begin{array}{c}\text { Recharge } \\
\text { (inches) }\end{array}$ \\
\hline 52 & 05380950 & Rock Ck at Greenwood & $44^{\circ} 45^{\prime} 30^{\prime \prime}$ & $90^{\circ} 35^{\prime} 52^{\prime \prime}$ & 05381000 & 77 & -- & relation & 16.3 & 2.9 \\
\hline 53 & 05380970 & Cawley Ck nr Neillsville & $44^{\circ} 36^{\prime} 41^{\prime \prime}$ & $90^{\circ} 34^{\prime} 29^{\prime \prime}$ & 05381000 & 38.2 & 0.0520 & relation & 7.2 & 2.6 \\
\hline 54 & 05380985 & S Br O'Neill Ck at Granton & $44^{\circ} 35^{\prime} 25^{\prime \prime}$ & $90^{\circ} 27^{\prime} 40^{\prime \prime}$ & 05381000 & 16.4 & -- & relation & 2.8 & 2.3 \\
\hline 55 & 05380990 & S Br O'Neill Ck nr Neillsville & $44^{\circ} 33^{\prime} 59^{\prime \prime}$ & $90^{\circ} 32^{\prime} 14^{\prime \prime}$ & 05381000 & 22.5 & -- & relation & 3.8 & 2.3 \\
\hline 56 & 05381055 & Cunningham Ck nr Neillsville & $44^{\circ} 31^{\prime} 01^{\prime \prime}$ & $90^{\circ} 34^{\prime} 42^{\prime \prime}$ & 05381000 & 44.4 & .0121 & equation & 4.4 & 1.3 \\
\hline 57 & 05381090 & Wedges Ck nr Neillsville & $44^{\circ} 34^{\prime} 24^{\prime \prime}$ & $90^{\circ} 43^{\prime} 21 "$ & 05381000 & 61.2 & .0489 & equation & 12.5 & 2.8 \\
\hline 58 & 05381110 & Wedges Ck nr Neillsville & $44^{\circ} 30^{\prime} 58^{\prime \prime}$ & $90^{\circ} 42^{\prime} 46^{\prime \prime}$ & 05381000 & 110 & -- & relation & 37.8 & 4.7 \\
\hline 59 & 05381150 & E Fk Black R nr Pittsville & $44^{\circ} 27^{\prime} 12^{\prime \prime}$ & $90^{\circ} 18^{\prime} 28^{\prime \prime}$ & 05381000 & 37 & .0050 & equation & 2.3 & .8 \\
\hline 60 & 05381167 & E Fk Black R nr Pray & $44^{\circ} 24^{\prime} 15^{\prime \prime}$ & $90^{\circ} 29^{\prime} 51^{\prime \prime}$ & 05381000 & 238 & -- & relation & 88.5 & 5.1 \\
\hline 61 & 05381170 & Rock Ck nr Neillsville & $44^{\circ} 26^{\prime} 21^{\prime \prime}$ & $90^{\circ} 35^{\prime} 54^{\prime \prime}$ & 05402000 & 27.3 & .0480 & equation & 5.5 & 2.7 \\
\hline 62 & 05381173 & E Fk Black R nr Hatfield & $44^{\circ} 25^{\prime} 04^{\prime \prime}$ & $90^{\circ} 38^{\prime} 22^{\prime \prime}$ & 05381000 & 292 & .0197 & equation & 38.5 & 1.8 \\
\hline 63 & 05381240 & Morrison Ck nr Hatfield & $44^{\circ} 22^{\prime} 40^{\prime \prime}$ & $90^{\circ} 43^{\prime} 16^{\prime \prime}$ & 05379500 & 104 & .0685 & equation & 25.7 & 3.4 \\
\hline 64 & 05381250 & Hay Ck nr Hatfield & $44^{\circ} 22^{\prime} 29^{\prime \prime}$ & $90^{\circ} 43^{\prime} 14^{\prime \prime}$ & 05379500 & 7.83 & .1206 & equation & 2.5 & 4.3 \\
\hline 65 & 05381260 & Dickey Ck nr Hatfield & $44^{\circ} 20^{\prime} 30^{\prime \prime}$ & $90^{\circ} 44^{\prime} 41^{\prime \prime}$ & 05379500 & 11.9 & .1238 & equation & 3.8 & 4.4 \\
\hline 66 & 05381283 & S Fk Halls Ck at Alma Center & $44^{\circ} 26^{\prime} 24^{\prime \prime}$ & $90^{\circ} 54^{\prime} 44^{\prime \prime}$ & 05379500 & 8.91 & -- & relation & 4.1 & 6.3 \\
\hline 67 & 05381285 & Halls Ck at Merrillan & $44^{\circ} 26^{\prime} 50^{\prime \prime}$ & $90^{\circ} 50^{\prime} 22^{\prime \prime}$ & 05379500 & 47.2 & -- & relation & 25.0 & 7.2 \\
\hline 68 & 05381290 & Halls Ck nr Black R Falls & $44^{\circ} 22^{\prime} 01^{\prime \prime}$ & $90^{\circ} 47^{\prime} 10^{\prime \prime}$ & 05379500 & 89.3 & .1837 & equation & 36.7 & 5.6 \\
\hline 69 & 05381350 & Levis Ck nr Black R Falls & $44^{\circ} 18^{\prime} 42^{\prime \prime}$ & $90^{\circ} 48^{\prime} 23^{\prime \prime}$ & 05379500 & 39 & .1860 & relation & 19.5 & 6.8 \\
\hline 70 & 05381400 & Robinson Ck nr Black R Falls & $44^{\circ} 11^{\prime} 48^{\prime \prime}$ & $90^{\circ} 50^{\prime} 21^{\prime \prime}$ & 05379500 & 108 & .3401 & equation & 61.4 & 7.7 \\
\hline 71 & 05381420 & Trout Run nr Melrose & $44^{\circ} 12^{\prime} 18^{\prime \prime}$ & $90^{\circ} 55^{\prime} 49^{\prime \prime}$ & 05379500 & 13.7 & .5232 & equation & 9.3 & 9.3 \\
\hline 72 & 05381450 & Big Ck nr Cataract & $44^{\circ} 08^{\prime} 08^{\prime \prime}$ & $90^{\circ} 54^{\prime} 26^{\prime \prime}$ & 05383000 & 64.6 & .6030 & relation & 57.8 & 12.2 \\
\hline 73 & 05381495 & Douglas $\mathrm{Ck}$ at Melrose & $44^{\circ} 07^{\prime} 56^{\prime \prime}$ & $90^{\circ} 59^{\prime} 35^{\prime \prime}$ & 05379500 & 22.8 & -- & relation & 16.9 & 10.1 \\
\hline 74 & 05381800 & Fleming $\mathrm{Ck}$ at Stevenstown & $44^{\circ} 02^{\prime} 17^{\prime \prime}$ & $91^{\circ} 10^{\prime} 09^{\prime \prime}$ & 05379500 & 39.5 & .3060 & relation & 16.1 & 5.5 \\
\hline 75 & 05382010 & Beaver Ck nr Taylor & $44^{\circ} 13^{\prime} 16^{\prime \prime}$ & $91^{\circ} 05^{\prime} 22^{\prime \prime}$ & 05379500 & 5.62 & .1811 & equation & 2.2 & 5.2 \\
\hline 76 & 05382022 & Beaver Ck nr Hegg & $44^{\circ} 12^{\prime} 43^{\prime \prime}$ & $91^{\circ} 09^{\prime} 26^{\prime \prime}$ & 05379500 & 17.8 & .2166 & equation & 7.7 & 5.9 \\
\hline 77 & 05382027 & N Fk Beaver Ck at Hegg & $44^{\circ} 12^{\prime} 38^{\prime \prime}$ & $91^{\circ} 10^{\prime} 40^{\prime \prime}$ & 05379500 & 20.1 & .2429 & equation & 9.3 & 6.3 \\
\hline
\end{tabular}

${ }^{1}$ Low-flow discharge measurements at partial-record stations were compared to one or more nearby USGS gaging stations to establish a relation between measured discharge and concurrent daily discharge. The nearby gaging station that provided the best relation with the partial-record station was chosen as the index station and was associated with nearby miscellaneous measurement sites. 
Appendix 6D. Average annual base flow and corresponding recharge, 1970-99, for partial-record stations in the Trempealeau-Black River Basin—Continued.

[USGS, U.S. Geological Survey; ID, identification number; $\mathrm{dd}^{\circ} \mathrm{mm}$ 'ss", degrees, minutes, seconds; $\mathrm{mi}^{2}$, square mile; $\mathrm{ft}^{3} / \mathrm{s} / \mathrm{mi}^{2}$, cubic foot per second per square mile; $\mathrm{ft}^{3} / \mathrm{s}$, cubic foot per second; equation, statewide regression equation; relation, established relation line with index station; R, River; Bk, Brook; Br, Branch; Fk, Fork; Ck, Creek; Tr, Tributary; nr, near; N, North; S, South; E, East; W, West; ,-- not determined in historical low-flow reports. See references for full list of low-flow reports.]

\begin{tabular}{|c|c|c|c|c|c|c|c|c|c|c|}
\hline $\begin{array}{c}\text { Map } \\
\text { number }\end{array}$ & $\begin{array}{c}\text { USGS } \\
\text { station ID }\end{array}$ & Station name & $\begin{array}{c}\text { Latitude } \\
\text { (ddºmm'ss") }\end{array}$ & $\begin{array}{c}\text { Longitude } \\
\text { (dd }{ }^{\circ} \mathrm{mm} \text { 'ss") }\end{array}$ & $\begin{array}{c}\text { Index } \\
\text { station }^{1}\end{array}$ & $\begin{array}{c}\text { Drainage } \\
\text { area } \\
\left(\mathrm{mi}^{2}\right) \\
\end{array}$ & $\begin{array}{c}\text { Base flow } \\
\text { factor } \\
\left(\mathrm{ft}^{3} / \mathrm{s} / \mathrm{mi}^{2}\right)\end{array}$ & $\begin{array}{c}\text { Base flow } \\
\text { method }\end{array}$ & $\begin{array}{c}\text { Base flow } \\
\left(\mathrm{ft}^{3} / \mathrm{s}\right)\end{array}$ & $\begin{array}{c}\text { Recharge } \\
\text { (inches) }\end{array}$ \\
\hline 78 & 05382044 & Bear Ck nr Beachs Corners & $44^{\circ} 13^{\prime} 21^{\prime \prime}$ & $91^{\circ} 14^{\prime} 21^{\prime \prime}$ & 05379500 & 3.01 & 0.3065 & equation & 1.5 & 6.8 \\
\hline 79 & 05382048 & Bear Ck Tr nr Beachs Corners & $44^{\circ} 13^{\prime} 16^{\prime \prime}$ & $91^{\circ} 14^{\prime} 37^{\prime \prime}$ & 05379500 & 2.53 & .1789 & equation & 1.0 & 5.1 \\
\hline 80 & 05382051 & Bear Ck at Beaches Corners & $44^{\circ} 12^{\prime} 37^{\prime \prime}$ & $91^{\circ} 14^{\prime} 21^{\prime \prime}$ & 05379500 & 7.21 & .2632 & equation & 3.4 & 6.4 \\
\hline 81 & 05382060 & N Fk Beaver Ck at Ettrick & $44^{\circ} 10^{\prime} 03^{\prime \prime}$ & $91^{\circ} 16^{\prime} 13^{\prime \prime}$ & 05379500 & 50.8 & -- & relation & 37.0 & 9.9 \\
\hline 82 & 05382071 & German Coulee nr N Bend & $44^{\circ} 09^{\prime} 56^{\prime \prime}$ & $91^{\circ} 07^{\prime} 10^{\prime \prime}$ & 05379500 & 2.67 & .0937 & equation & .7 & 3.7 \\
\hline 83 & 05382080 & S Fk Beaver Ck nr N Bend & $44^{\circ} 08^{\prime} 28^{\prime \prime}$ & $91^{\circ} 10^{\prime} 54^{\prime \prime}$ & 05379500 & 15.4 & .0896 & equation & 4.2 & 3.7 \\
\hline 84 & 05382087 & S Fk Beaver Ck Tr nr Ettrick & $44^{\circ} 09^{\prime} 12^{\prime \prime}$ & $91^{\circ} 12^{\prime} 36^{\prime \prime}$ & 05379500 & 5.3 & .1980 & equation & 2.1 & 5.5 \\
\hline 85 & 05382100 & S Fk Beaver Ck at Ettrick & $44^{\circ} 09^{\prime} 55^{\prime \prime}$ & $91^{\circ} 16^{\prime} 05^{\prime \prime}$ & 05379500 & 33.4 & .1640 & relation & 12.5 & 5.1 \\
\hline 86 & 05382120 & Beaver Ck nr Ettrick & $44^{\circ} 08^{\prime} 52^{\prime \prime}$ & $91^{\circ} 19^{\prime} 21^{\prime \prime}$ & 05379500 & 90.9 & .2547 & equation & 44.3 & 6.6 \\
\hline 87 & 05382130 & French Ck nr Ettrick & $44^{\circ} 13^{\prime} 32^{\prime \prime}$ & $91^{\circ} 19^{\prime} 25^{\prime \prime}$ & 05379500 & 2.13 & .3760 & equation & 1.2 & 7.5 \\
\hline 88 & 05382180 & French Ck Tr nr Ettrick & $44^{\circ} 11^{\prime} 31^{\prime \prime}$ & $91^{\circ} 18^{\prime} 22^{\prime \prime}$ & 05379500 & 2.23 & .1015 & equation & .6 & 3.8 \\
\hline 89 & 05382200 & French Ck nr Ettrick & $44^{\circ} 11^{\prime} 04^{\prime \prime}$ & $91^{\circ} 18^{\prime} 49^{\prime \prime}$ & 05379500 & 14.7 & .3170 & relation & 10.1 & 9.3 \\
\hline 90 & 05382204 & French Ck Tr nr Ettrick & $44^{\circ} 10^{\prime} 18^{\prime \prime}$ & $91^{\circ} 19^{\prime} 24^{\prime \prime}$ & 05379500 & 2.57 & .1524 & equation & .9 & 4.7 \\
\hline 91 & 05382206 & French Ck nr Ettrick & $44^{\circ} 08^{\prime} 57^{\prime \prime}$ & $91^{\circ} 19^{\prime} 30^{\prime \prime}$ & 05379500 & 22 & .2548 & equation & 10.4 & 6.4 \\
\hline 92 & 05382209 & Beaver Ck nr Galesville & $44^{\circ} 07^{\prime} 02^{\prime \prime}$ & $91^{\circ} 20^{\prime} 18^{\prime \prime}$ & 05379500 & 124 & .2560 & equation & 61.0 & 6.7 \\
\hline 93 & 05382211 & Beaver Ck at Galesville & $44^{\circ} 04^{\prime} 26^{\prime \prime}$ & $91^{\circ} 21^{\prime} 58^{\prime \prime}$ & 05379500 & 139 & -- & relation & 92.0 & 9.0 \\
\hline 94 & 05382237 & Halfway Ck at Holmen & $43^{\circ} 57^{\prime} 50^{\prime \prime}$ & $91^{\circ} 15^{\prime} 25^{\prime \prime}$ & 05379500 & 30.4 & -- & relation & 16.1 & 7.2 \\
\hline 95 & 05382265 & La Crosse R nr Angelo & $44^{\circ} 00^{\prime} 30^{\prime \prime}$ & $90^{\circ} 42^{\prime} 59^{\prime \prime}$ & 05379500 & 59.2 & -- & relation & 72.0 & 16.5 \\
\hline 96 & 05382280 & Silver Ck nr Sparta & $43^{\circ} 56^{\prime} 30^{\prime \prime}$ & $90^{\circ} 40^{\prime} 49^{\prime \prime}$ & 05382500 & 14.8 & .4500 & relation & 11.2 & 10.3 \\
\hline 97 & 05382330 & La Crosse R at Sparta & $43^{\circ} 56^{\prime} 11^{\prime \prime}$ & $90^{\circ} 48^{\prime} 39^{\prime \prime}$ & 05379500 & 167 & -- & relation & 164.5 & 13.4 \\
\hline 98 & 05382380 & Little La Crosse R at Cashton & $43^{\circ} 45^{\prime} 59^{\prime \prime}$ & $90^{\circ} 47^{\prime} 52^{\prime \prime}$ & 05408000 & 2.97 & -- & relation & 1.4 & 6.4 \\
\hline 99 & 05382410 & Little La Crosse $\mathrm{R}$ at Helvina & $43^{\circ} 48^{\prime} 21^{\prime \prime}$ & $90^{\circ} 46^{\prime} 15^{\prime \prime}$ & 05408000 & 18.3 & -- & relation & 8.5 & 6.3 \\
\hline 100 & 05382500 & Little La Crosse R nr Leon & $43^{\circ} 53^{\prime} 45^{\prime \prime}$ & $90^{\circ} 50^{\prime} 25^{\prime \prime}$ & 05379500 & 76.8 & .3322 & equation & 42.8 & 7.6 \\
\hline 101 & 05382560 & La Crosse R at Rockland & $43^{\circ} 54^{\prime} 48^{\prime \prime}$ & $90^{\circ} 55^{\prime} 11 "$ & 05379500 & 284 & -- & relation & 250.0 & 12.0 \\
\hline 102 & 05382590 & Dutch Ck nr Bangor & $43^{\circ} 52^{\prime} 41^{\prime \prime}$ & $90^{\circ} 59^{\prime} 53^{\prime \prime}$ & 05379500 & 16.4 & .4509 & equation & 10.4 & 8.6 \\
\hline 103 & 05382610 & La Crosse $\mathrm{R}$ at Bangor & $43^{\circ} 54^{\prime} 02^{\prime \prime}$ & $90^{\circ} 59^{\prime} 38^{\prime \prime}$ & 05379500 & 332 & -- & relation & 274.0 & 11.2 \\
\hline
\end{tabular}

${ }^{1}$ Low-flow discharge measurements at partial-record stations were compared to one or more nearby USGS gaging stations to establish a relation between measured discharge and concurrent daily discharge. The nearby gaging station that provided the best relation with the partial-record station was chosen as the index station and was associated with nearby miscellaneous measurement sites. 
Appendix 6D. Average annual base flow and corresponding recharge, 1970-99, for partial-record stations in the Trempealeau-Black River Basin-Continued.

[USGS, U.S. Geological Survey; ID, identification number; $\mathrm{dd}^{\circ} \mathrm{mm}$ 'ss", degrees, minutes, seconds; $\mathrm{mi}^{2}$, square mile; $\mathrm{ft}^{3} / \mathrm{s} / \mathrm{mi}^{2}$, cubic foot per second per square mile; $\mathrm{ft}^{3} / \mathrm{s}$, cubic foot per second; equation, statewide regression equation; relation, established relation line with index station; R, River; Bk, Brook; Br, Branch; Fk, Fork; Ck, Creek; Tr, Tributary; nr, near; N, North; S, South; E, East; W, West; --, not determined in historical low-flow reports. See references for full list of low-flow reports.]

\begin{tabular}{|c|c|c|c|c|c|c|c|c|c|c|}
\hline $\begin{array}{c}\text { Map } \\
\text { number }\end{array}$ & $\begin{array}{l}\text { USGS } \\
\text { station ID }\end{array}$ & Station name & $\begin{array}{c}\text { Latitude } \\
\left.\text { (dd }{ }^{\circ} \mathrm{mm} ' s s^{\prime \prime}\right)\end{array}$ & $\begin{array}{l}\text { Longitude } \\
\text { (dd'mm'ss") }\end{array}$ & $\begin{array}{l}\text { Index } \\
\text { station }{ }^{1}\end{array}$ & $\begin{array}{c}\text { Drainage } \\
\text { area } \\
\left(\mathrm{mi}^{2}\right)\end{array}$ & $\begin{array}{c}\text { Base flow } \\
\text { factor } \\
\left(\mathrm{ft}^{3} / \mathrm{s} / \mathrm{mi}^{2}\right)\end{array}$ & $\begin{array}{l}\text { Base flow } \\
\text { method }\end{array}$ & $\begin{array}{c}\text { Base flow } \\
\qquad\left(\mathrm{ft}^{3} / \mathrm{s}\right)\end{array}$ & $\begin{array}{c}\text { Recharge } \\
\text { (inches) }\end{array}$ \\
\hline 104 & 05383050 & Bostwick Ck nr Barre Mills & $43^{\circ} 51^{\prime} 53^{\prime \prime}$ & $91^{\circ} 07^{\prime} 37^{\prime \prime}$ & 05379500 & 40.3 & 0.2905 & equation & 20.7 & 7.0 \\
\hline 105 & 05386300 & Morman Ck nr La Crosse & $43^{\circ} 46^{\prime} 00^{\prime \prime}$ & $91^{\circ} 08^{\prime} 27^{\prime \prime}$ & 05382500 & 25.2 & .3800 & relation & 14.2 & 7.7 \\
\hline 106 & 05386850 & Coon $\mathrm{Ck}$ at Chaseburg & $43^{\circ} 39^{\prime} 28^{\prime \prime}$ & $91^{\circ} 05^{\prime} 22^{\prime \prime}$ & 05379500 & 104 & -- & relation & 59.0 & 7.7 \\
\hline 107 & 05387005 & Coon Ck nr Stoddard & $43^{\circ} 39^{\prime} 10^{\prime \prime}$ & $91^{\circ} 09^{\prime} 58^{\prime \prime}$ & 05379500 & 128 & .4184 & equation & 81.3 & 8.6 \\
\hline 108 & 05387090 & $\begin{array}{l}\text { Springville Br Bad Axe } \mathrm{R} \text { at } \\
\text { Springville }\end{array}$ & $43^{\circ} 35^{\prime} 00^{\prime \prime}$ & $90^{\circ} 56^{\prime} 20^{\prime \prime}$ & 05382500 & 8.73 & -- & relation & 4.4 & 6.8 \\
\hline 109 & 05387100 & N Fk Bad Axe R nr Genoa & $43^{\circ} 33^{\prime} 10^{\prime \prime}$ & $91^{\circ} 08^{\prime} 58^{\prime \prime}$ & 05408000 & 80.8 & .3000 & relation & 46.6 & 7.8 \\
\hline 110 & 05388370 & Rush Ck nr Ferryville & $43^{\circ} 23^{\prime} 22^{\prime \prime}$ & $91^{\circ} 07^{\prime} 36^{\prime \prime}$ & 05408500 & 44.8 & .2530 & relation & 33.0 & 10.0 \\
\hline 111 & 05388375 & Sugar Ck at Ferryville & $43^{\circ} 22^{\prime} 04^{\prime \prime}$ & $91^{\circ} 08^{\prime} 22^{\prime \prime}$ & 05408000 & 25.1 & -- & relation & 13.6 & 7.4 \\
\hline 112 & 05388380 & Copper Ck nr Mt Sterling & $43^{\circ} 18^{\prime} 58^{\prime \prime}$ & $90^{\circ} 59^{\prime} 59^{\prime \prime}$ & 05409890 & 14.2 & .3900 & relation & 8.7 & 8.3 \\
\hline
\end{tabular}

'Low-flow discharge measurements at partial-record stations were compared to one or more nearby USGS gaging stations to establish a relation between measured discharge and concurrent daily discharge. The nearby gaging station that provided the best relation with the partial-record station was chosen as the index station and was associated with nearby miscellaneous measurement sites. 


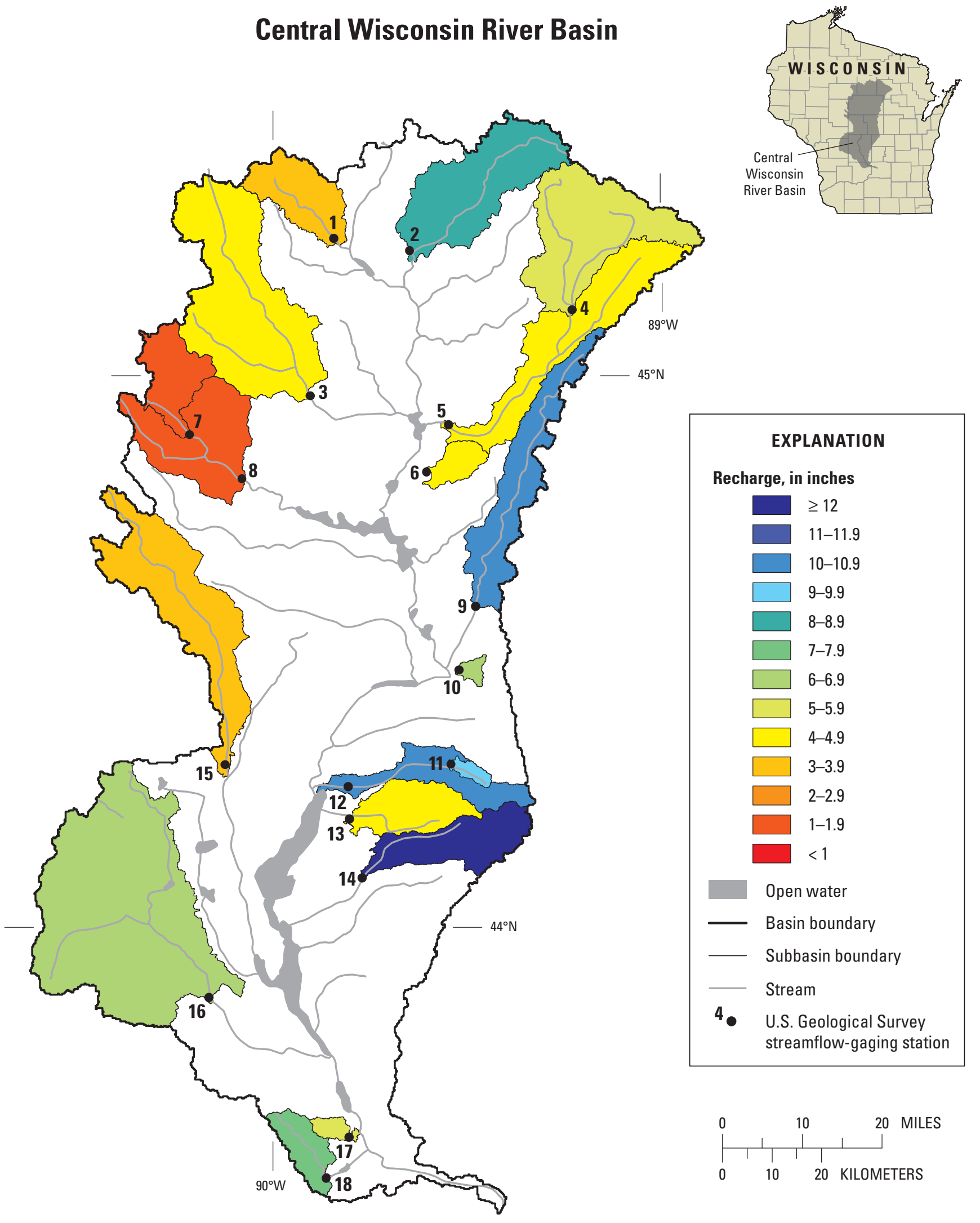

Appendix 7-A. Locations of streamflow-gaging stations in the Central Wisconsin River Basin and spatial distribution of average annual recharge. 


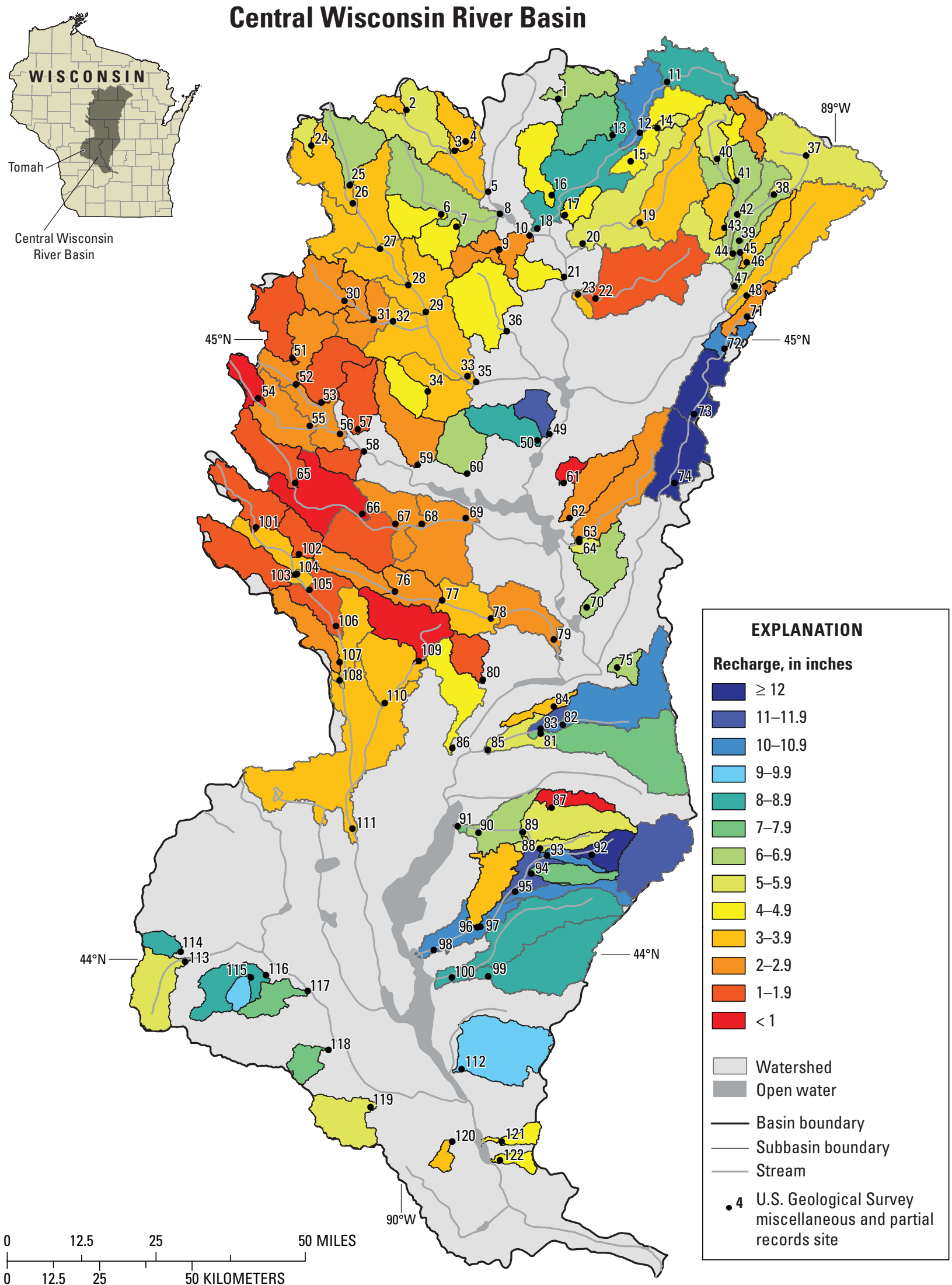

Appendix 7-B. Locations of miscellaneous and partial-record sites in the Central Wisconsin River Basin and spatial distribution of estimated average annual recharge. 
Appendix 7C. Average annual baseflow and corresponding recharge, 1970-99, for gaging stations in the Central Wisconsin River Basin.

[USGS, U.S. Geological Survey; ID, identification number; mi² $^{2}$ square mile; $\mathrm{ft}^{3}$ /s, cubic foot per second; BFI, Baseflow Index; ex rec, extended record; R, River; Bk, Brook; Br, Branch; Fk, Fork; Ck, Creek; Tr, Tributary; nr, near; N, North; S, South; E, East; W, West; --, not determined in historical low-flow reports. See references for full list of low-flow reports.]

\begin{tabular}{|c|c|c|c|c|c|c|c|c|c|c|c|}
\hline $\begin{array}{c}\text { Map } \\
\text { number }\end{array}$ & $\begin{array}{l}\text { USGS } \\
\text { station } \\
\text { ID }\end{array}$ & Station name & $\begin{array}{c}\text { Drainage } \\
\text { area } \\
\left(\mathrm{mi}^{2}\right)\end{array}$ & $\begin{array}{c}\text { Period of } \\
\text { analysis } \\
\text { (water years }{ }^{1} \text { ) }\end{array}$ & $\begin{array}{c}\text { Base flow } \\
\text { method }\end{array}$ & $\begin{array}{l}\text { Base flow } \\
\text { index }\end{array}$ & $\begin{array}{c}\text { Base flow } \\
\quad\left(\mathrm{ft}^{3} / \mathrm{s}\right)\end{array}$ & $\begin{array}{c}\text { Recharge } \\
\text { (inches) }\end{array}$ & $\begin{array}{c}\text { Total } \\
\text { streamflow } \\
\left(\mathrm{ft}^{3} / \mathrm{s}\right)\end{array}$ & $\begin{array}{c}\text { Total } \\
\text { streamflow } \\
\text { (inches) }\end{array}$ & $\begin{array}{l}{ }^{2} 090 \\
\left(\mathrm{ft}^{3} / \mathrm{s}\right)\end{array}$ \\
\hline 1 & 05394000 & New Wood R nr Merrill & 82.2 & $1953-1961$ & ex rec & -- & 22 & 3.6 & -- & -- & -- \\
\hline 2 & 05394500 & Prairie R nr Merrill & 184 & 1970-1999 & BFI & 0.6440 & 110 & 8.4 & 180 & 13.3 & 79 \\
\hline 3 & 05396000 & Rib R at Rib Falls & 303 & $1926-1957$ & ex rec & -- & 100 & 4.7 & -- & -- & 33 \\
\hline 4 & 05397110 & Eau Claire R nr Antigo & 185 & 1975-1981 & ex rec & -- & 72 & 5.3 & -- & -- & 3.7 \\
\hline 5 & 05397500 & Eau Claire R at Kelly & 375 & 1970-1999 & BFI & .5230 & 140 & 4.9 & 260 & 9.6 & 68 \\
\hline 6 & 05398500 & Bull Ck Jr nr Rothschild & 27.4 & $1945-1951$ & ex rec & -- & 10 & 5.0 & -- & -- & 4.4 \\
\hline 7 & 05399000 & Big Eau Pleine R nr Colby & 78.1 & $1942-1954$ & ex rec & -- & 7.4 & 1.3 & -- & -- & 1.4 \\
\hline 8 & 05399500 & Big Eau Pleine R at Stratford & 224 & 1970-1999 & BFI & .1850 & 32 & 1.9 & 180 & 11.1 & 6.8 \\
\hline 9 & 05400500 & Plover R nr Stevens Point & 145 & $\begin{array}{c}1915-1919 \\
1945-1951\end{array}$ & ex rec & -- & 120 & 10.8 & -- & -- & 85 \\
\hline 10 & 05400650 & Little Plover R at Plover & 19 & $1960-1987$ & ex rec & -- & 9.6 & 6.9 & -- & -- & 4.3 \\
\hline 11 & 05401020 & Tenmile Ck Ditch 5 nr Bancroft & 9.73 & $1965-1973$ & ex rec & -- & 7.0 & 9.8 & -- & -- & 3.7 \\
\hline 12 & 05401050 & Tenmile Ck nr Nekoosa & 73.3 & 1970-1999 & BFI & .8730 & 54 & 10.0 & 63 & 11.7 & 26 \\
\hline 13 & 05401100 & Fourteenmile Ck nr New Rome & 91.1 & $1965-1979$ & ex rec & -- & 31 & 4.6 & -- & -- & 7.6 \\
\hline 14 & 05401535 & Big Roche A Cri Ck nr Adams & 52.8 & 1964-1978 & ex rec & -- & 61 & 15.7 & -- & -- & 42 \\
\hline 15 & 05402000 & Yellow $\mathrm{R}$ at Babcock & 215 & 1970-1999 & BFI & .2580 & 48 & 3.0 & 190 & 12.1 & 10 \\
\hline 16 & 05403500 & Lemonweir $\mathrm{R}$ at New Lisbon & 507 & $1945-1987$ & ex rec & -- & 240 & 6.4 & -- & -- & 85 \\
\hline 17 & 05403630 & Hulbert Ck nr Wisconsin Dells & 11.2 & 1971-1977 & ex rec & -- & 4.2 & 5.1 & -- & -- & 2.8 \\
\hline 18 & 05403700 & Dell Ck nr Lake Delton & 44.9 & $1958-1980$ & ex rec & -- & 26 & 7.9 & -- & -- & 20 \\
\hline
\end{tabular}

${ }^{1}$ Water year is a 12-month period from October 1 through September 30, and is designated by the calendar year in which it ends (for example, the 2004 water year occurred October 1,2003 through September 30, 2004).

${ }^{2}$ Discharge that is exceeded 90 percent of the time. 
Appendix 7D. Average annual base flow and corresponding recharge, 1970-99, for partial-record stations in the Central Wisconsin River Basin.

[USGS, U.S. Geological Survey; ID, identification number; $\mathrm{dd}^{\circ} \mathrm{mm}$ 'ss", degrees, minutes, seconds; $\mathrm{mi}^{2}$, square mile; $\mathrm{ft}^{3} / \mathrm{s} / \mathrm{mi}^{2}$, cubic foot per second per square mile; $\mathrm{ft}^{3} / \mathrm{s}$, cubic foot per second; equation, statewide regression equation; relation, established relation line with index station; R, River; Bk, Brook; Br, Branch; Fk, Fork; Ck, Creek; Tr, Tributary; nr, near; N, North; S, South; E, East; W, West; --, not determined in historical low-flow reports. See references for full list of low-flow reports.]

\begin{tabular}{|c|c|c|c|c|c|c|c|c|c|c|}
\hline $\begin{array}{l}\text { Map } \\
\text { number }\end{array}$ & $\begin{array}{l}\text { USGS } \\
\text { station ID }\end{array}$ & Station name & $\begin{array}{c}\text { Latitude } \\
\text { (dd }{ }^{\circ} \mathrm{mm} \text { 'ss") }\end{array}$ & $\begin{array}{l}\text { Longitude } \\
\text { (dd }{ }^{\circ} \mathrm{mm} \text { 'ss") }\end{array}$ & $\begin{array}{l}\text { Index } \\
\text { station }\end{array}$ & $\begin{array}{c}\text { Drainage } \\
\text { area } \\
\left(\mathrm{mi}^{2}\right)\end{array}$ & $\begin{array}{c}\text { Base flow } \\
\text { factor } \\
\left(\mathrm{ft}^{3} / \mathrm{s} / \mathrm{mi}^{2}\right)\end{array}$ & $\begin{array}{l}\text { Base flow } \\
\text { method }\end{array}$ & $\begin{array}{l}\text { Base flow } \\
\qquad\left(\mathrm{ft}^{3} / \mathrm{s}\right)\end{array}$ & $\begin{array}{c}\text { Recharge } \\
\text { (inches) }\end{array}$ \\
\hline 1 & 05393630 & Little Pine Ck nr Tomahawk & $45^{\circ} 23^{\prime} 30^{\prime \prime}$ & $89^{\circ} 39^{\prime} 05^{\prime \prime}$ & 05394500 & 21 & 0.2390 & relation & 9.9 & 6.4 \\
\hline 2 & 05393850 & $\begin{array}{l}\text { Center Fk New Wood R nr Spirit } \\
\text { Falls }\end{array}$ & $45^{\circ} 22^{\prime} 24^{\prime \prime}$ & $89^{\circ} 59^{\prime} 56^{\prime \prime}$ & 05393500 & 5.43 & .0721 & equation & 1.3 & 3.2 \\
\hline 3 & 05393910 & New Wood R nr Irma & $45^{\circ} 18^{\prime} 28^{\prime \prime}$ & $89^{\circ} 53^{\prime} 21^{\prime \prime}$ & 05393500 & 53 & .1360 & relation & 19.2 & 4.9 \\
\hline 4 & 05393916 & Averill Ck nr New Wood & $45^{\circ} 19^{\prime} 23^{\prime \prime}$ & $89^{\circ} 51^{\prime} 49^{\prime \prime}$ & 05393500 & 10.6 & .0711 & equation & 2.5 & 3.3 \\
\hline 5 & 05394002 & New Wood R nr Merrill & $45^{\circ} 14^{\prime} 31^{\prime \prime}$ & $89^{\circ} 48^{\prime} 45^{\prime \prime}$ & 05393500 & 85.9 & .0709 & equation & 21.5 & 3.4 \\
\hline 6 & 05394110 & M Fk Copper R nr Hamburg & $45^{\circ} 12^{\prime} 22^{\prime \prime}$ & $89^{\circ} 55^{\prime} 12^{\prime \prime}$ & 05393500 & 16.4 & .1526 & equation & 5.9 & 4.9 \\
\hline 7 & 05394130 & S Fk Copper R nr Hamburg & $45^{\circ} 11^{\prime} 07^{\prime \prime}$ & $89^{\circ} 53^{\prime} 06^{\prime \prime}$ & 05393500 & 15.8 & .1183 & equation & 5.0 & 4.3 \\
\hline 8 & 05394160 & Copper R nr Hamburg & $45^{\circ} 12^{\prime} 23^{\prime \prime}$ & $89^{\circ} 47^{\prime} 10^{\prime \prime}$ & 05393500 & 94.4 & .1250 & relation & 39.3 & 5.7 \\
\hline 9 & 05394200 & Devil Ck nr Merrill & $45^{\circ} 08^{\prime} 56^{\prime \prime}$ & $89^{\circ} 47^{\prime} 13^{\prime \prime}$ & 05393500 & 9.58 & .1320 & relation & 2.0 & 2.8 \\
\hline 10 & 05394207 & Devil Ck at Merrill & $45^{\circ} 10^{\prime} 15^{\prime \prime}$ & $89^{\circ} 43^{\prime} 07^{\prime \prime}$ & 05393500 & 27.1 & .0548 & equation & 5.8 & 2.9 \\
\hline 11 & 05394250 & Prairie $\mathrm{R}$ at Parrish & $45^{\circ} 25^{\prime} 04^{\prime \prime}$ & $89^{\circ} 24^{\prime} 06^{\prime \prime}$ & 05394500 & 37.3 & .4199 & equation & 23.2 & 8.4 \\
\hline 12 & 05394310 & Prairie R nr Gleason & $45^{\circ} 20^{\prime} 10^{\prime \prime}$ & $89^{\circ} 27^{\prime} 53^{\prime \prime}$ & 05394500 & 61.4 & .6206 & equation & 47.2 & 10.4 \\
\hline 13 & 05394380 & N Br Prairie R nr Gleason & $45^{\circ} 19^{\prime} 57^{\prime \prime}$ & $89^{\circ} 31^{\prime} 37^{\prime \prime}$ & 05394500 & 34.8 & .2790 & relation & 20.3 & 7.9 \\
\hline 14 & 05394420 & Silver Ck nr Parrish & $45^{\circ} 20^{\prime} 39^{\prime \prime}$ & $89^{\circ} 25^{\prime} 27^{\prime \prime}$ & 05394500 & 4.08 & .1790 & equation & 1.6 & 5.2 \\
\hline 15 & 05394450 & Big Hay Meadow Ck nr Gleason & $45^{\circ} 17^{\prime} 24^{\prime \prime}$ & $89^{\circ} 29^{\prime} 10^{\prime \prime}$ & 05394500 & 32.3 & .1507 & equation & 11.7 & 4.9 \\
\hline 16 & 05394750 & Meadow Ck nr Merrill & $45^{\circ} 14^{\prime} 08^{\prime \prime}$ & $89^{\circ} 40^{\prime} 02^{\prime \prime}$ & 05394500 & 23.8 & .1290 & equation & 7.9 & 4.5 \\
\hline 17 & 05394768 & Barnes Ck nr Merrill & $45^{\circ} 12^{\prime} 13^{\prime \prime}$ & $89^{\circ} 38^{\prime} 14^{\prime \prime}$ & 05394500 & 9.9 & .1147 & equation & 3.0 & 4.2 \\
\hline 18 & 05394797 & Prairie $\mathrm{Ck}$ at Merrill & $45^{\circ} 10^{\prime} 55^{\prime \prime}$ & $89^{\circ} 42^{\prime} 01^{\prime \prime}$ & 05394500 & 231 & .4046 & equation & 145.9 & 8.6 \\
\hline 19 & 05395030 & Pine R nr Merrill & $45^{\circ} 11^{\prime} 27^{\prime \prime}$ & $89^{\circ} 27^{\prime} 58^{\prime \prime}$ & 05394500 & 55.2 & .0520 & relation & 13.5 & 3.3 \\
\hline 20 & 05395063 & Pine R nr Merrill & $45^{\circ} 09^{\prime} 26^{\prime \prime}$ & $89^{\circ} 35^{\prime} 50^{\prime \prime}$ & 05394500 & 118 & .1114 & equation & 37.6 & 4.3 \\
\hline 21 & 05395068 & County Line Ck nr Merrill & $45^{\circ} 06^{\prime} 14^{\prime \prime}$ & $89^{\circ} 38^{\prime} 24^{\prime \prime}$ & 05394500 & 12.7 & .1285 & equation & 4.2 & 4.5 \\
\hline 22 & 05395130 & Trappe R nr Wausau & $45^{\circ} 04^{\prime} 09^{\prime \prime}$ & $89^{\circ} 34^{\prime} 07^{\prime \prime}$ & 05394500 & 73.6 & .0310 & relation & 8.1 & 1.5 \\
\hline 23 & 053951312 & Trappe R nr Brokaw & $45^{\circ} 04^{\prime} 31^{\prime \prime}$ & $89^{\circ} 36^{\prime} 30^{\prime \prime}$ & 05394500 & 79.2 & .0791 & equation & 21.0 & 3.6 \\
\hline 24 & 05395504 & Sheep Ranch Ck at Rib Lk & $45^{\circ} 18^{\prime} 59^{\prime \prime}$ & $90^{\circ} 13^{\prime} 01^{\prime \prime}$ & 05393500 & 7.84 & .1160 & relation & 3.2 & 5.5 \\
\hline 25 & 05395550 & Wood Ck nr Rib Lk & $45^{\circ} 15^{\prime} 07^{\prime \prime}$ & $90^{\circ} 07^{\prime} 44^{\prime \prime}$ & 05396000 & 31.4 & .2280 & relation & 15.1 & 6.5 \\
\hline
\end{tabular}

${ }^{1}$ Low-flow discharge measurements at partial-record stations were compared to one or more nearby USGS gaging stations to establish a relation between measured discharge and concurrent daily discharge. The nearby gaging station that provided the best relation with the partial-record station was chosen as the index station and was associated with nearby miscellaneous measurement sites. 
[USGS, U.S. Geological Survey; ID, identification number; $\mathrm{dd}^{\circ} \mathrm{mm}$ 'ss", degrees, minutes, seconds; $\mathrm{mi}^{2}$, square mile; $\mathrm{ft}^{3} / \mathrm{s} / \mathrm{mi}^{2}$, cubic foot per second per square mile; $\mathrm{ft}^{3} / \mathrm{s}$, cubic foot per second; equation, statewide regression equation; relation, established relation line with index station; R, River; Bk, Brook; Br, Branch; Fk, Fork; Ck, Creek; Tr, Tributary; nr, near; N, North; S, South; E, East; W, West; ,-- not determined in historical low-flow reports. See references for full list of low-flow reports.]

\begin{tabular}{|c|c|c|c|c|c|c|c|c|c|c|}
\hline $\begin{array}{l}\text { Map } \\
\text { number }\end{array}$ & $\begin{array}{c}\text { USGS } \\
\text { station ID }\end{array}$ & Station name & $\begin{array}{c}\text { Latitude } \\
\text { (ddºmm'ss") }\end{array}$ & $\begin{array}{l}\text { Longitude } \\
\text { (dd }{ }^{\circ} \mathrm{mm} \text { 'ss") }\end{array}$ & $\begin{array}{l}\text { Index } \\
\text { station }{ }^{1}\end{array}$ & $\begin{array}{c}\text { Drainage } \\
\text { area } \\
\left(\mathrm{mi}^{2}\right)\end{array}$ & $\begin{array}{c}\text { Base flow } \\
\text { factor } \\
\left(\mathrm{ft}^{3} / \mathrm{s} / \mathrm{mi}^{2}\right)\end{array}$ & $\begin{array}{l}\text { Base flow } \\
\text { method }\end{array}$ & $\begin{array}{c}\text { Base flow } \\
\qquad\left(\mathrm{ft}^{3} / \mathrm{s}\right)\end{array}$ & $\begin{array}{c}\text { Recharge } \\
\text { (inches) }\end{array}$ \\
\hline 26 & 05395580 & Rib R nr Goodrich & $45^{\circ} 13^{\prime} 24^{\prime \prime}$ & $90^{\circ} 07^{\prime} 16^{\prime \prime}$ & 05393500 & 71.8 & 0.0941 & equation & 20.8 & 3.9 \\
\hline 27 & 05395650 & Rib R at Goodrich & $45^{\circ} 08^{\prime} 59^{\prime \prime}$ & $90^{\circ} 03^{\prime} 34^{\prime \prime}$ & 05393500 & 131 & .0866 & equation & 36.7 & 3.8 \\
\hline 28 & 05395700 & Rib R nr Hamburg & $45^{\circ} 05^{\prime} 27^{\prime \prime}$ & $89^{\circ} 59^{\prime} 41^{\prime \prime}$ & 05393500 & 159 & .0790 & equation & 42.6 & 3.6 \\
\hline 29 & 05395750 & Rib R nr athens & $45^{\circ} 02^{\prime} 51^{\prime \prime}$ & $89^{\circ} 57^{\prime} 17^{\prime \prime}$ & 05393500 & 193 & .0792 & equation & 52.0 & 3.7 \\
\hline 30 & 05395850 & Black Ck above Beaver Ck & $45^{\circ} 03^{\prime} 58^{\prime \prime}$ & $90^{\circ} 08^{\prime} 23^{\prime \prime}$ & 05399500 & 25.4 & .0296 & equation & 3.9 & 2.1 \\
\hline 31 & 05395870 & Black Ck at athens & $45^{\circ} 02^{\prime} 09^{\prime \prime}$ & $90^{\circ} 04^{\prime} 24^{\prime \prime}$ & 05399500 & 39.9 & .0780 & relation & 8.2 & 2.8 \\
\hline 32 & 05395900 & Black Ck nr athens & $45^{\circ} 01^{\prime} 58^{\prime \prime}$ & $90^{\circ} 01^{\prime} 48^{\prime \prime}$ & 05393500 & 58.8 & .0331 & equation & 9.8 & 2.3 \\
\hline 33 & 05396015 & Rib R nr Marathon & $44^{\circ} 56^{\prime} 39^{\prime \prime}$ & $89^{\circ} 51^{\prime} 36^{\prime \prime}$ & 05393500 & 316 & .0696 & equation & 80.4 & 3.5 \\
\hline 34 & 05396060 & Scotch Ck at Edgar & $44^{\circ} 55^{\prime} 12^{\prime \prime}$ & $89^{\circ} 57^{\prime} 02^{\prime \prime}$ & 05399500 & 16.9 & .0470 & relation & 5.2 & 4.2 \\
\hline 35 & 05396200 & Rib R at Marathon & $44^{\circ} 56^{\prime} 06^{\prime \prime}$ & $89^{\circ} 50^{\prime} 25^{\prime \prime}$ & 05393500 & 365 & .1250 & relation & 85.3 & 3.2 \\
\hline 36 & 05396400 & Little Rib R nr Hamburg & $45^{\circ} 01^{\prime} 01^{\prime \prime}$ & $89^{\circ} 46^{\prime} 14^{\prime \prime}$ & 05397500 & 51 & .1408 & equation & 18.1 & 4.8 \\
\hline 37 & 05396830 & E Br Eau Claire R nr Kempster & $45^{\circ} 17^{\prime} 48^{\prime \prime}$ & $89^{\circ} 05^{\prime} 06^{\prime \prime}$ & 05397500 & 38.6 & .0040 & equation & 6.4 & 2.3 \\
\hline 38 & 05396920 & E Br Eau Claire R at Deerbrook & $45^{\circ} 14^{\prime} 02^{\prime \prime}$ & $89^{\circ} 09^{\prime} 33^{\prime \prime}$ & 05397500 & 65.7 & .2430 & relation & 26.0 & 5.4 \\
\hline 39 & 05397015 & E Br Eau Claire R nr Antigo & $45^{\circ} 09^{\prime} 39^{\prime \prime}$ & $89^{\circ} 14^{\prime} 24^{\prime \prime}$ & 05397500 & 87.2 & -- & relation & 37.0 & 5.8 \\
\hline 40 & 05397025 & W Br Eau Claire R nr Kempster & $45^{\circ} 17^{\prime} 33^{\prime \prime}$ & $89^{\circ} 17^{\prime} 18^{\prime \prime}$ & 05397500 & 17 & .1722 & equation & 6.5 & 5.2 \\
\hline 41 & 05397030 & Sucker Cr nr Dempster & $45^{\circ} 15^{\prime} 28^{\prime \prime}$ & $89^{\circ} 14^{\prime} 42^{\prime \prime}$ & 05397500 & 6.93 & .1305 & equation & 2.3 & 4.4 \\
\hline 42 & 05397045 & W Br Eau Claire R nr Antigo & $45^{\circ} 12^{\prime} 08^{\prime \prime}$ & $89^{\circ} 14^{\prime} 37^{\prime \prime}$ & 05397500 & 51.9 & .2565 & equation & 25.1 & 6.6 \\
\hline 43 & 05397085 & Black Bk Tr nr Antigo & $45^{\circ} 10^{\prime} 54^{\prime \prime}$ & $89^{\circ} 16^{\prime} 22^{\prime \prime}$ & 05397500 & 11.4 & .0787 & equation & 2.9 & 3.4 \\
\hline 44 & 05397090 & W Br Eau Claire R nr Antigo & $45^{\circ} 08^{\prime} 25^{\prime \prime}$ & $89^{\circ} 15^{\prime} 17^{\prime \prime}$ & 05397500 & 89.7 & .1290 & relation & 27.6 & 4.2 \\
\hline 45 & 05397100 & Eau Claire R nr Antigo & $45^{\circ} 08^{\prime} 29^{\prime \prime}$ & $89^{\circ} 14^{\prime} 15^{\prime \prime}$ & 05397500 & 179 & .2030 & relation & 86.9 & 6.6 \\
\hline 46 & 05397120 & Eau Claire R Tr nr Antigo & $45^{\circ} 07^{\prime} 33^{\prime \prime}$ & $89^{\circ} 13^{\prime} 24^{\prime \prime}$ & 05397500 & 15 & .0834 & equation & 3.9 & 3.6 \\
\hline 47 & 05397140 & Eau Claire R nr Antigo & $45^{\circ} 05^{\prime} 13^{\prime \prime}$ & $89^{\circ} 15^{\prime} 06^{\prime \prime}$ & 05397500 & 215 & .2309 & equation & 101.3 & 6.4 \\
\hline 48 & 05397200 & Spring Bk nr Antigo & $45^{\circ} 04^{\prime} 18^{\prime \prime}$ & $89^{\circ} 13^{\prime} 26^{\prime \prime}$ & 05397500 & 79.2 & .1380 & relation & 22.3 & 3.8 \\
\hline 49 & 05398100 & Black Ck nr Mosinee & $44^{\circ} 51^{\prime} 04^{\prime \prime}$ & $89^{\circ} 40^{\prime} 29^{\prime \prime}$ & 05399500 & 13 & .0880 & relation & 11.4 & 11.9 \\
\hline 50 & 05398233 & Fourmile Ck nr Mosinee & $44^{\circ} 50^{\prime} 26^{\prime \prime}$ & $89^{\circ} 42^{\prime} 08^{\prime \prime}$ & 05397500 & 24.3 & .4011 & equation & 14.6 & 8.2 \\
\hline 51 & 05398800 & $\begin{array}{l}\text { W Br Big Eau Pleine R nr } \\
\text { Abbotsford }\end{array}$ & $44^{\circ} 58^{\prime} 25^{\prime \prime}$ & $90^{\circ} 15^{\prime} 33^{\prime \prime}$ & 05399500 & 33.1 & .0175 & equation & 3.9 & 1.6 \\
\hline
\end{tabular}

${ }^{1}$ Low-flow discharge measurements at partial-record stations were compared to one or more nearby USGS streamflow-gaging stations to establish a relation between measured discharge and concurrent daily discharge. The nearby gaging station that provided the best relation with the partial-record station was chosen as the index station and was associated with nearby miscellaneous measurement sites. 
Appendix 7D. Average annual base flow and corresponding recharge, 1970-99, for partial-record stations in the Central Wisconsin River Basin-Continued.

[USGS, U.S. Geological Survey; ID, identification number; $\mathrm{dd}^{\circ} \mathrm{mm}$ 'ss", degrees, minutes, seconds; $\mathrm{mi}^{2}$, square mile; $\mathrm{ft}^{3} / \mathrm{s} / \mathrm{mi}^{2}$, cubic foot per second per square mile; $\mathrm{ft}^{3} / \mathrm{s}$, cubic foot per second; equation, statewide regression equation; relation, established relation line with index station; R, River; Bk, Brook; Br, Branch; Fk, Fork; Ck, Creek; Tr, Tributary; nr, near; N, North; S, South; E, East; W, West; --, not determined in historical low-flow reports. See references for full list of low-flow reports.]

\begin{tabular}{|c|c|c|c|c|c|c|c|c|c|c|}
\hline $\begin{array}{c}\text { Map } \\
\text { number }\end{array}$ & $\begin{array}{c}\text { USGS } \\
\text { station ID }\end{array}$ & Station name & $\begin{array}{c}\text { Latitude } \\
\text { (dd }{ }^{\circ} \mathrm{mm} \text { 'ss") }\end{array}$ & $\begin{array}{c}\text { Longitude } \\
\text { (dd }{ }^{\circ} \mathrm{mm} \text { 'ss") }\end{array}$ & $\begin{array}{l}\text { Index } \\
\text { station } 1\end{array}$ & $\begin{array}{c}\text { Drainage } \\
\text { area } \\
\left(\mathrm{mi}^{2}\right)\end{array}$ & $\begin{array}{c}\text { Base flow } \\
\text { factor } \\
\left(\mathrm{ft}^{3} / \mathrm{s} / \mathrm{mi}^{2}\right)\end{array}$ & $\begin{array}{c}\text { Base flow } \\
\text { method }\end{array}$ & $\begin{array}{c}\text { Base flow } \\
\quad\left(\mathrm{ft}^{3} / \mathrm{s}\right)\end{array}$ & $\begin{array}{c}\text { Recharge } \\
\text { (inches) }\end{array}$ \\
\hline 52 & 05398950 & Big Eau Pleine R nr Abbotsford & $44^{\circ} 55^{\prime} 52^{\prime \prime}$ & $90^{\circ} 15^{\prime} 02^{\prime \prime}$ & 05399500 & 73.1 & 0.0228 & equation & 10.1 & 1.9 \\
\hline 53 & 05399210 & Randall Ck nr Colby & $44^{\circ} 54^{\prime} 07^{\prime \prime}$ & $90^{\circ} 11^{\prime} 33^{\prime \prime}$ & 05399500 & 30.9 & .0181 & equation & 3.7 & 1.6 \\
\hline 54 & 05399250 & Dill Ck at Colby & $44^{\circ} 54^{\prime} 28^{\prime \prime}$ & $90^{\circ} 20^{\prime} 09^{\prime \prime}$ & 05399500 & 12.3 & .0670 & relation & .7 & .8 \\
\hline 55 & 05399350 & Dill Ck nr Unity & $44^{\circ} 51^{\prime} 50^{\prime \prime}$ & $90^{\circ} 13^{\prime} 06^{\prime \prime}$ & 05399500 & 46.7 & .0251 & equation & 6.7 & 2.0 \\
\hline 56 & 05399400 & Big Ea Pleine R nr Stratford & $44^{\circ} 51^{\prime} 05^{\prime \prime}$ & $90^{\circ} 09^{\prime} 00^{\prime \prime}$ & 05399500 & 174 & .0307 & equation & 28.6 & 2.2 \\
\hline 57 & 05399448 & Hamann Ck nr Stratford & $44^{\circ} 51^{\prime} 31^{\prime \prime}$ & $90^{\circ} 06^{\prime} 35^{\prime \prime}$ & 05399500 & 25.8 & .0182 & equation & 3.1 & 1.6 \\
\hline 58 & 05399479 & Noisy Ck nr Stratford & $44^{\circ} 49^{\prime} 21^{\prime \prime}$ & $90^{\circ} 05^{\prime} 44^{\prime \prime}$ & 05399500 & 12.7 & .0102 & equation & 1.1 & 1.2 \\
\hline 59 & 05399550 & Fenwood Ck nr Stratford & $44^{\circ} 48^{\prime} 03^{\prime \prime}$ & $89^{\circ} 58^{\prime} 25^{\prime \prime}$ & 05399500 & 36.9 & .0421 & equation & 6.9 & 2.6 \\
\hline 60 & 05399580 & Freeman Ck nr Halder & $44^{\circ} 47^{\prime} 11^{\prime \prime}$ & $89^{\circ} 51^{\prime} 44^{\prime \prime}$ & 05399500 & 26.5 & .1210 & relation & 12.0 & 6.2 \\
\hline 61 & 05400005 & Peplin Ck nr Mosinee & $44^{\circ} 46^{\prime} 18^{\prime \prime}$ & $89^{\circ} 38^{\prime} 34^{\prime \prime}$ & 05397500 & 6.37 & .0030 & equation & .3 & 6 \\
\hline 62 & 05400030 & Johnson Ck nr Knowlton & $44^{\circ} 42^{\prime} 51^{\prime \prime}$ & $89^{\circ} 37^{\prime} 46^{\prime \prime}$ & 05397500 & 28.6 & .0515 & equation & 5.9 & 2.8 \\
\hline 63 & 05400040 & Little Eau Claire R nr Knowlton & $44^{\circ} 40^{\prime} 49^{\prime \prime}$ & $89^{\circ} 36^{\prime} 28^{\prime \prime}$ & 05397500 & 49.2 & .0274 & equation & 7.4 & 2.1 \\
\hline 64 & 05400041 & Little Eau Clair R Tr nr Dancy & $44^{\circ} 40^{\prime} 34^{\prime \prime}$ & $89^{\circ} 36^{\prime} 28^{\prime \prime}$ & 05397500 & 3.95 & .2045 & equation & 1.6 & 5.5 \\
\hline 65 & 05400050 & Little Eau Pleine R nr Spencer & $44^{\circ} 46^{\prime} 18^{\prime \prime}$ & $90^{\circ} 15^{\prime} 05^{\prime \prime}$ & 05399500 & 28.2 & .0142 & equation & 3.0 & 1.4 \\
\hline 66 & 05400100 & Little Eau Plein R nr Marshfield & $44^{\circ} 43^{\prime} 20^{\prime \prime}$ & $90^{\circ} 05^{\prime} 58^{\prime \prime}$ & 05399500 & 78 & .0070 & relation & 4.4 & .8 \\
\hline 67 & 05400150 & Little Eau Pleine R nr Rozeville & $44^{\circ} 42^{\prime} 21^{\prime \prime}$ & $90^{\circ} 01^{\prime} 27^{\prime \prime}$ & 05399500 & 119 & .0225 & equation & 16.5 & 1.9 \\
\hline 68 & 05400200 & Little Eau Pleine R nr Auburndale & $44^{\circ} 42^{\prime} 21^{\prime \prime}$ & $89^{\circ} 57^{\prime} 51^{\prime \prime}$ & 05399500 & 147 & .0266 & equation & 22.4 & 2.1 \\
\hline 69 & 05400250 & Little Eau Pleine R nr Millador & $44^{\circ} 42^{\prime} 53^{\prime \prime}$ & $89^{\circ} 51^{\prime} 55^{\prime \prime}$ & 05399500 & 200 & .0390 & equation & 37.3 & 2.5 \\
\hline 70 & 05400300 & Hay Meadow Ck nr Stevens Point & $44^{\circ} 34^{\prime} 12^{\prime \prime}$ & $89^{\circ} 35^{\prime} 32^{\prime \prime}$ & 05398500 & 30.4 & -- & relation & 14.3 & 6.4 \\
\hline 71 & 05400330 & Plover R nr Aniwa & $45^{\circ} 02^{\prime} 18^{\prime \prime}$ & $89^{\circ} 13^{\prime} 26^{\prime \prime}$ & 05397500 & 6.86 & .0528 & relation & 1.4 & 2.8 \\
\hline 72 & 05400370 & Plover R nr Aniwa & $44^{\circ} 59^{\prime} 11^{\prime \prime}$ & $89^{\circ} 16^{\prime} 33^{\prime \prime}$ & 05397500 & 19.6 & .6754 & equation & 15.4 & 10.7 \\
\hline 73 & 05400410 & Plover R at Hatley & $44^{\circ} 52^{\prime} 54^{\prime \prime}$ & $89^{\circ} 20^{\prime} 46^{\prime \prime}$ & 05397500 & 47 & .7847 & equation & 40.6 & 11.7 \\
\hline 74 & 05400440 & Plover $\mathrm{R}$ at Bevent & $44^{\circ} 46^{\prime} 15^{\prime \prime}$ & $89^{\circ} 23^{\prime} 30^{\prime \prime}$ & 05397500 & 80 & .8667 & equation & 73.5 & 12.5 \\
\hline 75 & 05400645 & Little Plover R nr Plover & $44^{\circ} 28^{\prime} 22^{\prime \prime}$ & $89^{\circ} 31^{\prime} 26^{\prime \prime}$ & 05400650 & 18.1 & -- & relation & 9.3 & 7.0 \\
\hline 76 & 05400670 & Mill Ck nr Auburndale & $44^{\circ} 35^{\prime} 48^{\prime \prime}$ & $90^{\circ} 01^{\prime} 30^{\prime \prime}$ & 05399500 & 20.1 & .4769 & equation & 4.3 & 2.9 \\
\hline 77 & 05400685 & Mill Ck at Sherry & $44^{\circ} 34^{\prime} 57^{\prime \prime}$ & $89^{\circ} 55^{\prime} 07^{\prime \prime}$ & 05399500 & 41.6 & .0316 & equation & 6.7 & 2.2 \\
\hline
\end{tabular}

${ }^{1}$ Low-flow discharge measurements at partial-record stations were compared to one or more nearby USGS gaging stations to establish a relation between measured discharge and concurrent daily discharge. The nearby gaging station that provided the best relation with the partial-record station was chosen as the index station and was associated with nearby miscellaneous measurement sites. 
Appendix 7D. Average annual base flow and corresponding recharge, 1970-99, for partial-record stations in the Central Wisconsin River Basin-Continued.

[USGS, U.S. Geological Survey; ID, identification number; $\mathrm{dd}^{\circ} \mathrm{mm}$ 'ss", degrees, minutes, seconds; $\mathrm{mi}^{2}$, square mile; $\mathrm{ft}^{3} / \mathrm{s} / \mathrm{mi}^{2}$, cubic foot per second per square mile; $\mathrm{ft}^{3} / \mathrm{s}$, cubic foot per second; equation, statewide regression equation; relation, established relation line with index station; R, River; Bk, Brook; Br, Branch; Fk, Fork; Ck, Creek; Tr, Tributary; nr, near; N, North; S, South; E, East; W, West; ,-- not determined in historical low-flow reports. See references for full list of low-flow reports.]

\begin{tabular}{|c|c|c|c|c|c|c|c|c|c|c|}
\hline $\begin{array}{c}\text { Map } \\
\text { number }\end{array}$ & $\begin{array}{c}\text { USGS } \\
\text { station ID }\end{array}$ & Station name & $\begin{array}{c}\text { Latitude } \\
\text { (ddºmm'ss") }\end{array}$ & $\begin{array}{c}\text { Longitude } \\
\text { (dd }{ }^{\circ} \mathrm{mm} \text { 'ss") }\end{array}$ & $\begin{array}{l}\text { Index } \\
\text { station }^{1}\end{array}$ & $\begin{array}{c}\text { Drainage } \\
\text { area } \\
\left(\mathrm{mi}^{2}\right)\end{array}$ & $\begin{array}{l}\text { Base flow } \\
\text { factor } \\
\left(\mathrm{ft}^{3} / \mathrm{s} / \mathrm{mi}^{2}\right)\end{array}$ & $\begin{array}{l}\text { Base flow } \\
\text { method }\end{array}$ & $\begin{array}{c}\text { Base flow } \\
\quad\left(\mathrm{ft}^{3} / \mathrm{s}\right)\end{array}$ & $\begin{array}{c}\text { Recharge } \\
\text { (inches) }\end{array}$ \\
\hline 78 & 05400700 & Mill Ck at Junction City & $44^{\circ} 33^{\prime} 10^{\prime \prime}$ & $89^{\circ} 48^{\prime} 28^{\prime \prime}$ & 05399500 & 68.7 & 0.0350 & relation & 17.0 & 3.4 \\
\hline 79 & 05400710 & Mill Ck nr Stevens Point & $44^{\circ} 31^{\prime} 08^{\prime \prime}$ & $89^{\circ} 39^{\prime} 59^{\prime \prime}$ & 05399500 & 102 & .0542 & equation & 22.3 & 3.0 \\
\hline 80 & 05400750 & Mosquito Ck nr Rudolph & $44^{\circ} 27^{\prime} 16^{\prime \prime}$ & $89^{\circ} 49^{\prime} 38^{\prime \prime}$ & 05400650 & 16.3 & .0172 & equation & 1.9 & 1.6 \\
\hline 81 & 05400840 & Four Mile Ck nr Keller & $44^{\circ} 22^{\prime} 04^{\prime \prime}$ & $89^{\circ} 41^{\prime} 52^{\prime \prime}$ & 05401050 & 75 & .2850 & relation & 41.9 & 7.6 \\
\hline 82 & 05400850 & Buena Vista Cr nr Wisconsin Rapids & $44^{\circ} 22^{\prime} 54^{\prime \prime}$ & $89^{\circ} 38^{\prime} 51^{\prime \prime}$ & 05401050 & 45.7 & .3620 & relation & 34.0 & 10.1 \\
\hline 83 & 05400853 & Buena Vista Cr nr Wisconsin Rapids & $44^{\circ} 22^{\prime} 26^{\prime \prime}$ & $89^{\circ} 41^{\prime} 52^{\prime \prime}$ & 05401050 & 53.1 & .7420 & relation & 45.0 & 11.5 \\
\hline 84 & 05400885 & Twomile Ck nr Meehan & $44^{\circ} 24^{\prime} 38^{\prime \prime}$ & $89^{\circ} 40^{\prime} 03^{\prime \prime}$ & 05401050 & 2.79 & .0902 & equation & .7 & 3.6 \\
\hline 85 & 05400905 & Fourmile $\mathrm{Ck}$ at Wisconsin Rapids & $44^{\circ} 20^{\prime} 26^{\prime \prime}$ & $89^{\circ} 50^{\prime} 44^{\prime \prime}$ & 05401050 & 165 & .1388 & equation & 59.4 & 4.9 \\
\hline 86 & 05400950 & Moccasin Ck at Nekoosa & $44^{\circ} 20^{\prime} 37^{\prime \prime}$ & $89^{\circ} 53^{\prime} 45^{\prime \prime}$ & 05402000 & 22.3 & .1880 & relation & 8.1 & 4.9 \\
\hline 87 & 05401075 & Ditch N0 7 nr New Rome & $44^{\circ} 14^{\prime} 49^{\prime \prime}$ & $89^{\circ} 40^{\prime} 24^{\prime \prime}$ & 05401050 & 10.8 & .0042 & equation & .6 & .8 \\
\hline 88 & 05401085 & Fourteenmile Ck Tr nr Hancock & $44^{\circ} 10^{\prime} 52^{\prime \prime}$ & $89^{\circ} 41^{\prime} 58^{\prime \prime}$ & 05401050 & 9.58 & .1974 & equation & 3.9 & 5.5 \\
\hline 89 & 05401095 & Fourteenmile Ck nr New Rome & $44^{\circ} 12^{\prime} 29^{\prime \prime}$ & $89^{\circ} 44^{\prime} 15^{\prime \prime}$ & 05401050 & 77 & .1395 & equation & 27.4 & 4.8 \\
\hline 90 & 05401110 & Fourteenmile Ck nr New Rome & $44^{\circ} 12^{\prime} 26^{\prime \prime}$ & $89^{\circ} 50^{\prime} 15^{\prime \prime}$ & 05401050 & 98.8 & .2663 & equation & 49.4 & 6.8 \\
\hline 91 & 05401149 & Fourteenmile $\mathrm{Ck}$ at New Rome & $44^{\circ} 13^{\prime} 04^{\prime \prime}$ & $89^{\circ} 53^{\prime} 03^{\prime \prime}$ & 05401050 & 102 & .3484 & equation & 58.7 & 7.8 \\
\hline 92 & 05401510 & Big Roche at Chi Crk nr Handcock & $44^{\circ} 10^{\prime} 15^{\prime \prime}$ & $89^{\circ} 34^{\prime} 59^{\prime \prime}$ & 05401535 & 9.61 & .4880 & relation & 9.9 & 14.0 \\
\hline 93 & 05401516 & Big Roche A Cri Ck nr Hancock & $44^{\circ} 10^{\prime} 13^{\prime \prime}$ & $89^{\circ} 41^{\prime} 03^{\prime \prime}$ & 05401535 & 23.2 & .6527 & equation & 17.9 & 10.5 \\
\hline 94 & 05401520 & Big Roche A Ck Tr nr Hancock & $44^{\circ} 08^{\prime} 26^{\prime \prime}$ & $89^{\circ} 43^{\prime} 09^{\prime \prime}$ & 05401535 & 15.7 & .3703 & equation & 9.0 & 7.8 \\
\hline 95 & 05401530 & Big Roche A Cri Ck nr Friendship & $44^{\circ} 06^{\prime} 43^{\prime \prime}$ & $89^{\circ} 45^{\prime} 22^{\prime \prime}$ & 05400650 & 51.4 & .4230 & relation & 44.7 & 11.8 \\
\hline 96 & 05401545 & Big Roche A Cri Ck nr Arkdale & $44^{\circ} 03^{\prime} 18^{\prime \prime}$ & $89^{\circ} 50^{\prime} 02^{\prime \prime}$ & 05401535 & 107 & .5867 & equation & 80.8 & 10.3 \\
\hline 97 & 05401550 & Dead Horse Ck nr Arkdale & $44^{\circ} 03^{\prime} 15^{\prime \prime}$ & $89^{\circ} 50^{\prime} 25^{\prime \prime}$ & 05401535 & 30.7 & .0720 & equation & 7.6 & 3.4 \\
\hline 98 & 05401558 & Big Roche A Cri Ck nr Arkdale & $44^{\circ} 01^{\prime} 04^{\prime \prime}$ & $89^{\circ} 56^{\prime} 16^{\prime \prime}$ & 05401535 & 151 & .6064 & equation & 116.7 & 10.5 \\
\hline 99 & 05401580 & Little Roche A Cri Ck at Friendship & $43^{\circ} 58^{\prime} 30^{\prime \prime}$ & $89^{\circ} 49^{\prime} 00^{\prime \prime}$ & 05401535 & 57.5 & .4650 & relation & 36.9 & 8.7 \\
\hline 100 & 05401608 & Little Roche A Cri Ck nr Adams & $43^{\circ} 58^{\prime} 25^{\prime \prime}$ & $89^{\circ} 53^{\prime} 52^{\prime \prime}$ & 05401535 & 133 & .4055 & equation & 83.2 & 8.5 \\
\hline 101 & 05401630 & Yellow R nr Spencer & $44^{\circ} 41^{\prime} 57^{\prime \prime}$ & $90^{\circ} 20^{\prime} 25^{\prime \prime}$ & 05399500 & 27.6 & .0171 & equation & 3.2 & 1.6 \\
\hline 102 & 05401700 & E Br Yellow R nr Marshfield & $44^{\circ} 39^{\prime} 22^{\prime \prime}$ & $90^{\circ} 14^{\prime} 33^{\prime \prime}$ & 05381000 & 15.5 & .0130 & relation & 1.4 & 1.2 \\
\hline 103 & 05401710 & Yellow R nr Marshfield & $44^{\circ} 37^{\prime} 27^{\prime \prime}$ & $90^{\circ} 14^{\prime} 47^{\prime \prime}$ & 05402000 & 62.9 & .0266 & equation & 9.4 & 2.0 \\
\hline
\end{tabular}

${ }^{1}$ Low-flow discharge measurements at partial-record stations were compared to one or more nearby USGS gaging stations to establish a relation between measured discharge and concurrent daily discharge. The nearby gaging station that provided the best relation with the partial-record station was chosen as the index station and was associated with nearby miscellaneous measurement sites. 
Appendix 7D. Average annual base flow and corresponding recharge, 1970-99, for partial-record stations in the Central Wisconsin River Basin-Continued.

[USGS, U.S. Geological Survey; ID, identification number; $\mathrm{dd}^{\circ} \mathrm{mm}$ 'ss", degrees, minutes, seconds; $\mathrm{mi}^{2}$, square mile; $\mathrm{ft}^{3} / \mathrm{s} / \mathrm{mi}^{2}$, cubic foot per second per square mile; $\mathrm{ft}^{3} / \mathrm{s}$, cubic foot per second; equation, statewide regression equation; relation, established relation line with index station; R, River; Bk, Brook; Br, Branch; Fk, Fork; Ck, Creek; Tr, Tributary; nr, near; N, North; S, South; E, East; W, West; ,-- not determined in historical low-flow reports. See references for full list of low-flow reports.]

\begin{tabular}{|c|c|c|c|c|c|c|c|c|c|c|}
\hline$\underset{\text { Mumber }}{\text { Map }}$ & $\begin{array}{c}\text { USGS } \\
\text { station ID }\end{array}$ & Station name & $\begin{array}{c}\text { Latitude } \\
\text { (dd }{ }^{\circ} \mathrm{mm} \text { 'ss") }\end{array}$ & $\begin{array}{l}\text { Longitude } \\
\text { (ddºmm'ss") }\end{array}$ & $\begin{array}{l}\text { Index } \\
\text { station }^{1}\end{array}$ & $\begin{array}{c}\text { Drainage } \\
\text { area } \\
\left(\mathrm{mi}^{2}\right)\end{array}$ & $\begin{array}{c}\text { Base flow } \\
\text { factor } \\
\left(\mathrm{ft}^{3} / \mathrm{s} / \mathrm{mi}^{2}\right)\end{array}$ & $\begin{array}{c}\text { Base flow } \\
\text { method }\end{array}$ & $\begin{array}{c}\text { Base flow } \\
\quad\left(\mathrm{ft}^{3} / \mathrm{s}\right)\end{array}$ & $\begin{array}{c}\text { Recharge } \\
\text { (inches) }\end{array}$ \\
\hline 104 & 05401750 & S Br Yellow R nr Marshfield & $44^{\circ} 37^{\prime} 24^{\prime \prime}$ & $90^{\circ} 15^{\prime} 03^{\prime \prime}$ & 05381000 & 36 & 0.0340 & relation & 3.1 & 1.2 \\
\hline 105 & 05401755 & Yellow R nr Bakerville & $44^{\circ} 35^{\prime} 55^{\prime \prime}$ & $90^{\circ} 13^{\prime} 06^{\prime \prime}$ & 05402000 & 102 & .0192 & relation & 30.0 & 4.0 \\
\hline 106 & 05401770 & Yellow R nr Arpin & $44^{\circ} 32^{\prime} 26^{\prime \prime}$ & $90^{\circ} 09^{\prime} 33^{\prime \prime}$ & 05402000 & 128 & .0228 & equation & 17.9 & 1.9 \\
\hline 107 & 05401880 & Rocky Ck nr Pittsville & $44^{\circ} 28^{\prime} 57^{\prime \prime}$ & $90^{\circ} 09^{\prime} 02^{\prime \prime}$ & 05402000 & 21.9 & .0274 & equation & 3.3 & 2.0 \\
\hline 108 & 05401890 & Yellow R nr Pittsville & $44^{\circ} 27^{\prime} 12^{\prime \prime}$ & $90^{\circ} 09^{\prime} 00^{\prime \prime}$ & 05402000 & 180 & .0053 & relation & 44.0 & 3.3 \\
\hline 109 & 05402170 & Hemlock Ck at Vesper & $44^{\circ} 29^{\prime} 04^{\prime \prime}$ & $89^{\circ} 58^{\prime} 17^{\prime \prime}$ & 05399500 & 41.2 & .0010 & relation & 1.3 & .4 \\
\hline 110 & 05402300 & Hemlock Ck nr Dexterville & $44^{\circ} 25^{\prime} 00^{\prime \prime}$ & $90^{\circ} 02^{\prime} 51^{\prime \prime}$ & 05402000 & 77.2 & .0197 & equation & 9.9 & 1.7 \\
\hline 111 & 05402400 & Yellow R at Finley & $44^{\circ} 12^{\prime} 50^{\prime \prime}$ & $90^{\circ} 07^{\prime} 12^{\prime \prime}$ & 05402000 & 379 & .0502 & equation & 81.7 & 2.9 \\
\hline 112 & 05403250 & White Ck nr Friendship & $43^{\circ} 49^{\prime} 32^{\prime \prime}$ & $89^{\circ} 52^{\prime} 35^{\prime \prime}$ & 05401535 & 63.8 & .4280 & relation & 46.9 & 10.0 \\
\hline 113 & 05403300 & S Fk Lemonweir R at Tomah & $43^{\circ} 59^{\prime} 52^{\prime \prime}$ & $90^{\circ} 29^{\prime} 41^{\prime \prime}$ & 05403500 & 35.4 & .1080 & relation & 13.5 & 5.2 \\
\hline 114 & 05403308 & Mud Ck at Tomah & $44^{\circ} 00^{\prime} 50^{\prime \prime}$ & $90^{\circ} 30^{\prime} 18^{\prime \prime}$ & 05405000 & 7.04 & .4340 & relation & 4.2 & 8.1 \\
\hline 115 & 05403396 & Allen Ck nr Oakdale & $43^{\circ} 58^{\prime} 24^{\prime \prime}$ & $90^{\circ} 20^{\prime} 52^{\prime \prime}$ & 05403500 & 7.44 & .1200 & relation & 5.0 & 9.1 \\
\hline 116 & 05403400 & Bear Ck nr Camp Douglas & $43^{\circ} 58^{\prime} 36^{\prime \prime}$ & $90^{\circ} 18^{\prime} 47^{\prime \prime}$ & 05403500 & 33.2 & .1590 & relation & 20.6 & 8.4 \\
\hline 117 & 05403480 & Lemonweir R Tr at Camp Williams & $43^{\circ} 57^{\prime} 04^{\prime \prime}$ & $90^{\circ} 13^{\prime} 11^{\prime \prime}$ & 05403500 & 14.1 & .2870 & relation & 7.8 & 7.5 \\
\hline 118 & 05403520 & Webster Ck nr New Lisbon & $43^{\circ} 51^{\prime} 23^{\prime \prime}$ & $90^{\circ} 10^{\prime} 25^{\prime \prime}$ & 05408000 & 11.6 & .2460 & relation & 6.1 & 7.1 \\
\hline 119 & 05403550 & One Mile Ck nr Mauston & $43^{\circ} 45^{\prime} 50^{\prime \prime}$ & $90^{\circ} 04^{\prime} 45^{\prime \prime}$ & 05405000 & 30.4 & .3440 & relation & 12.0 & 5.4 \\
\hline 120 & 05403576 & Lyndon Ck at Lyndon Station & $43^{\circ} 42^{\prime} 33^{\prime \prime}$ & $89^{\circ} 42^{\prime} 33^{\prime \prime}$ & 05401535 & 6.11 & .2130 & relation & 1.5 & 3.3 \\
\hline 121 & 05403586 & Plainville Ck nr Wisconsin Dells & $43^{\circ} 42^{\prime} 32^{\prime \prime}$ & $89^{\circ} 47^{\prime} 15^{\prime \prime}$ & 05401535 & 6.9 & .1520 & relation & 2.3 & 4.5 \\
\hline 122 & 05403601 & Witches Gulch nr Wisconsin Dells & $43^{\circ} 40^{\prime} 42^{\prime \prime}$ & $89^{\circ} 47^{\prime} 30^{\prime \prime}$ & 05401535 & 6.76 & .1281 & equation & 2.2 & 4.4 \\
\hline
\end{tabular}

${ }^{1}$ Low-flow discharge measurements at partial-record stations were compared to one or more nearby USGS gaging stations to establish a relation between measured discharge and concurrent daily discharge. The nearby gaging station that provided the best relation with the partial-record station was chosen as the index station and was associated with nearby miscellaneous measurement sites. 


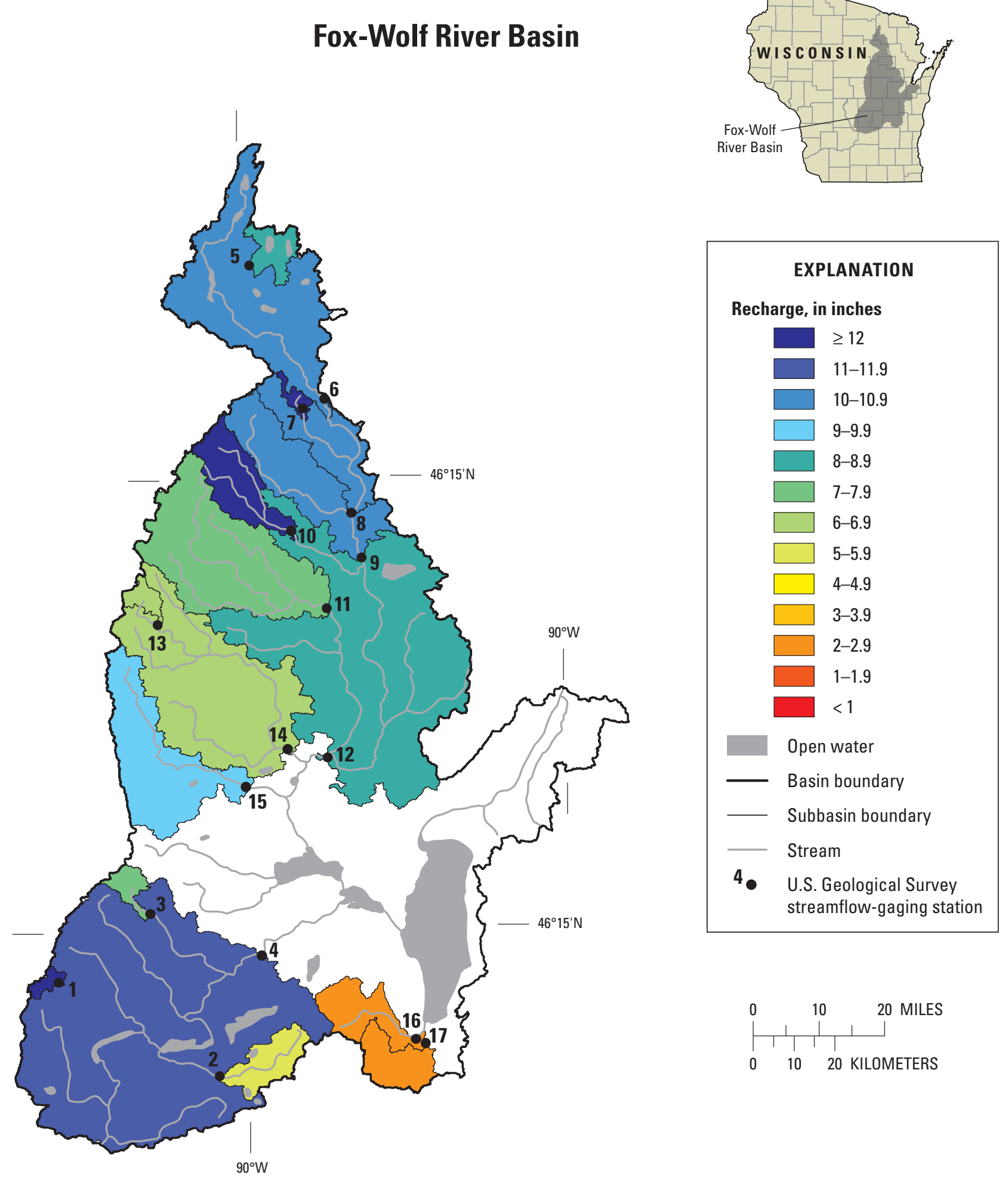

Appendix 8-A. Locations of streamflow-gaging stations in the Fox-Wolf River Basin and spatial distribution of average annual recharge. 


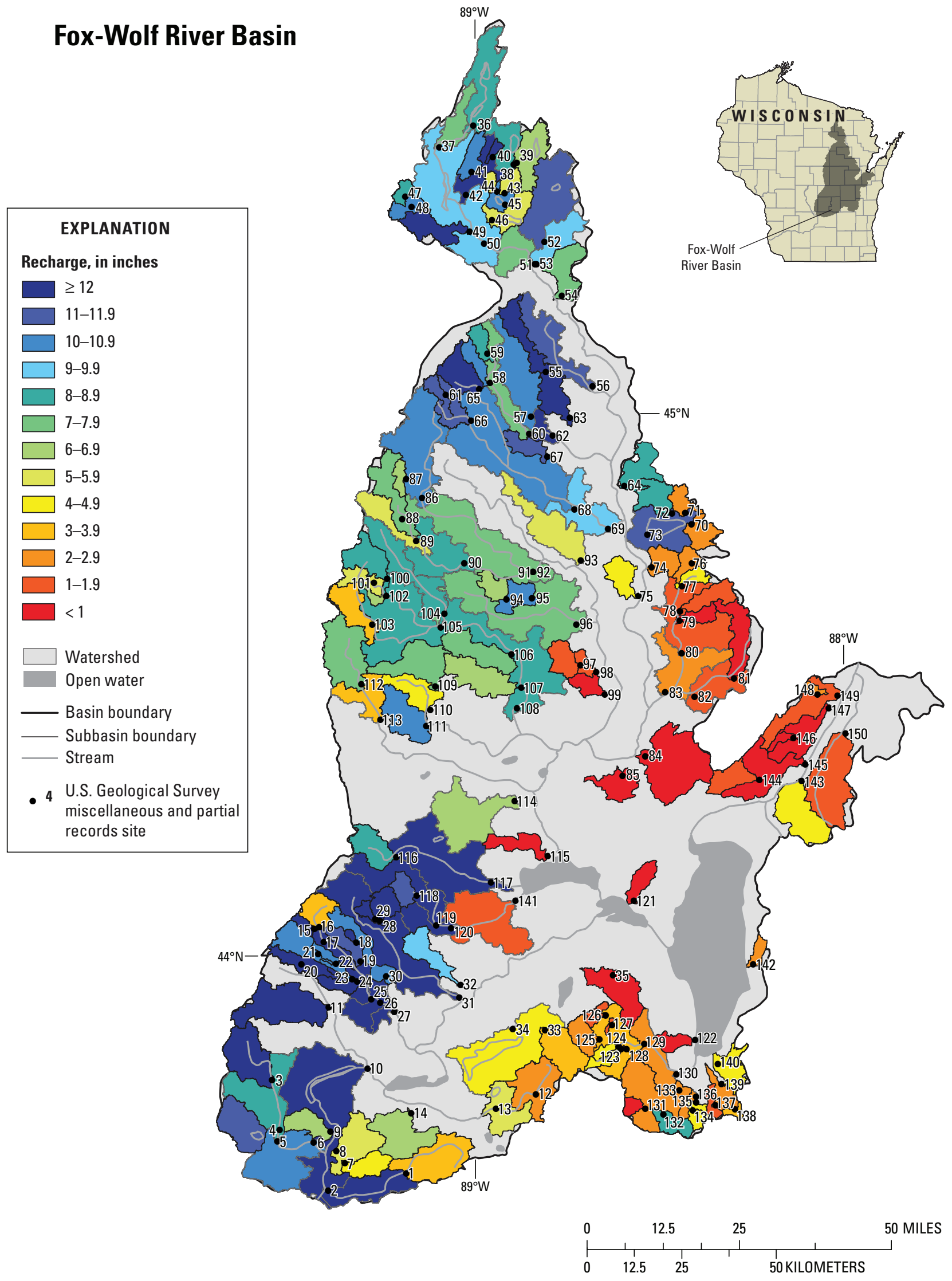

Appendix 8-B. Locations of miscellaneous and partial-record sites in the Fox-Wolf River Basin and spatial distribution of estimated average annual recharge. 
[USGS, U.S. Geological Survey; ID, identification number; $\mathrm{mi}^{2}$, square mile; $\mathrm{ft}^{3} / \mathrm{s}$, cubic foot per second; BFI, Baseflow Index; ex rec, extended record; R, River; Bk, Brook; Br, Branch; Fk, Fork; Ck, Creek; Tr, Tributary; nr, near; N, North; S, South; E, East; W, West; --, not determined in historical low-flow reports. See references for full list of low-flow reports.]

\begin{tabular}{|c|c|c|c|c|c|c|c|c|c|c|c|}
\hline $\begin{array}{c}\text { Map } \\
\text { number }\end{array}$ & $\begin{array}{l}\text { USGS } \\
\text { station } \\
\text { ID } \\
\end{array}$ & Station name & $\begin{array}{c}\text { Drainage } \\
\text { area } \\
\left(\mathrm{mi}^{2}\right)\end{array}$ & $\begin{array}{c}\text { Period of } \\
\text { analysis } \\
\text { (water years }{ }^{1} \text { ) }\end{array}$ & $\begin{array}{l}\text { Base flow } \\
\text { method }\end{array}$ & $\begin{array}{l}\text { Base flow } \\
\text { index }\end{array}$ & $\begin{array}{l}\text { Base flow } \\
\quad\left(\mathrm{ft}^{3} / \mathrm{s}\right)\end{array}$ & $\begin{array}{c}\text { Recharge } \\
\text { (inches) }\end{array}$ & $\begin{array}{c}\text { Total } \\
\text { streamflow } \\
\left(\mathrm{ft}^{3} / \mathrm{s}\right)\end{array}$ & $\begin{array}{c}\text { Total } \\
\text { streamflow } \\
\text { (inches) }\end{array}$ & $\begin{array}{r}{ }^{2} 090 \\
\left(\mathrm{ft}^{3} / \mathrm{s}\right)\end{array}$ \\
\hline 1 & 04072750 & Lawrence Ck nr Westfield & 13.44 & $1969-1973$ & ex rec & -- & 16 & 16.7 & -- & -- & -- \\
\hline 2 & 04073050 & Grand R nr Kingston & 73.5 & $1969-1975$ & ex rec & -- & 28 & 5.2 & -- & -- & -- \\
\hline 3 & 04073405 & W Br White R nr Wautoma & 38.9 & $1964-1965$ & ex rec & -- & 21 & 7.3 & -- & -- & -- \\
\hline 4 & 04073500 & Fox $\mathrm{R}$ at Berlin & 1,340 & 1970-1999 & BFI & 0.8720 & 1,100 & 11.4 & 1,300 & 13.0 & 560 \\
\hline 5 & 04074538 & Swamp Ck at Mole Lake & 46.3 & $1978-1986$ & ex rec & -- & 29 & 8.5 & -- & -- & 14 \\
\hline 6 & 04074950 & Wolf $\mathrm{R}$ at Langlade & 468 & 1970-1999 & BFI & .7820 & 350 & 10.2 & 450 & 13.1 & 240 \\
\hline 7 & 04075200 & Evergreen Ck nr Langlade & 8.09 & $1968-1973$ & ex rec & -- & 10 & 17.6 & -- & -- & 8.2 \\
\hline 8 & 04075500 & Wolf $\mathrm{R}$ nr Keshena & 616 & $1928-1962$ & ex rec & -- & 460 & 10.1 & -- & -- & 310 \\
\hline 9 & 04077400 & Wolf R nr Shawano & 816 & 1970-1999 & BFI & .7950 & 620 & 10.3 & 780 & 13.0 & 420 \\
\hline 10 & 04077630 & Red R nr Morgan & 114 & 1970-1999 & ex rec & -- & 110 & 13.1 & -- & -- & 74 \\
\hline 11 & 04078500 & Embarrass R nr Embarrass & 384 & 1970-1999 & BFI & .6220 & 200 & 7.2 & 330 & 11.7 & 110 \\
\hline 12 & 04079000 & Wolf $\mathrm{R}$ at New London & 2,260 & 1970-1999 & BFI & .7560 & 1,470 & 8.8 & 1,900 & 11.7 & 790 \\
\hline 13 & 04079602 & Little Wolf R nr Galloway & 22.6 & 1974-1979 & ex rec & -- & 11 & 6.4 & -- & -- & 6.9 \\
\hline 14 & 04080000 & Little Wolf R at Royalton & 507 & $1915-1985$ & ex rec & -- & 240 & 6.4 & -- & -- & 140 \\
\hline 15 & 04081000 & Waupaca R nr Waupaca & 265 & $1917-1985$ & ex rec & -- & 180 & 9.1 & -- & -- & 120 \\
\hline 16 & 04083000 & $\begin{array}{l}\text { W Br Fond Du Lac R at Fond Du } \\
\text { Lac }\end{array}$ & 83.1 & $1940-1954$ & ex rec & -- & 14 & 2.2 & -- & -- & -- \\
\hline 17 & 04083500 & E Br Fond Du Lac R at Fond Du Lac & 78.4 & $1940-1954$ & ex rec & -- & 14 & 2.5 & -- & -- & 4.0 \\
\hline
\end{tabular}

${ }^{1}$ Water year is a 12-month period from October 1 through September 30, and is designated by the calendar year in which it ends (for example, the 2004 water year occurred October 1, 2003 through September 30, 2004).

${ }^{2}$ Discharge that is exceeded 90 percent of the time. 
Appendix 8D. Average annual base flow and corresponding recharge, 1970-99, for partial-record stations in the Fox-Wolf River Basin.

[USGS, U.S. Geological Survey; ID, identification number; $\mathrm{dd}^{\circ} \mathrm{mm}$ 'ss", degrees, minutes, seconds; $\mathrm{mi}^{2}$, square mile; $\mathrm{ft}^{3} / \mathrm{s} / \mathrm{mi}^{2}$, cubic foot per second per square mile; $\mathrm{ft}^{3} / \mathrm{s}$, cubic foot per second; equation, statewide regression equation; relation, established relation line with index station; R, River; Bk, Brook; Br, Branch; Fk, Fork; Ck, Creek; Tr, Tributary; nr, near; N, North; S, South; E, East; W, West; ,-- not determined in historical low-flow reports. See references for full list of low-flow reports.]

\begin{tabular}{|c|c|c|c|c|c|c|c|c|c|c|}
\hline $\begin{array}{c}\text { Map } \\
\text { number }\end{array}$ & $\begin{array}{c}\text { USGS } \\
\text { station ID }\end{array}$ & Station name & $\begin{array}{c}\text { Latitude } \\
\text { (dd }{ }^{\circ} \mathrm{mm} \text { 'ss") }\end{array}$ & $\begin{array}{c}\text { Longitude } \\
\text { (dd }{ }^{\circ} \mathrm{mm} \text { 'ss") }\end{array}$ & $\begin{array}{l}\text { Index } \\
\text { station }{ }^{1}\end{array}$ & $\begin{array}{c}\text { Drainage } \\
\text { area } \\
\left(\mathrm{mi}^{2}\right)\end{array}$ & $\begin{array}{l}\text { Base flow } \\
\text { factor } \\
\left(\mathrm{ft}^{3} / \mathrm{s} / \mathrm{mi}^{2}\right)\end{array}$ & $\begin{array}{c}\text { Base flow } \\
\text { method }\end{array}$ & $\begin{array}{c}\text { Base flow } \\
\qquad\left(\mathrm{ft}^{3} / \mathrm{s}\right)\end{array}$ & $\begin{array}{c}\text { Recharge } \\
\text { (inches) }\end{array}$ \\
\hline 1 & 04072400 & Fox R nr Pardeeville & $43^{\circ} 35^{\prime} 04^{\prime \prime}$ & $89^{\circ} 13^{\prime} 39^{\prime \prime}$ & 04073500 & 41 & 0.0720 & relation & 10.5 & 3.5 \\
\hline 2 & 04072480 & Fox R nr Portage & $43^{\circ} 33^{\prime} 16^{\prime \prime}$ & $89^{\circ} 25^{\prime} 53^{\prime \prime}$ & 04073500 & 71.9 & .2597 & relation & 39.0 & 7.4 \\
\hline 3 & 04072660 & Neenah Ck nr Oxford & $43^{\circ} 45^{\prime} 50^{\prime \prime}$ & $89^{\circ} 34^{\prime} 36^{\prime \prime}$ & 04081000 & 33.9 & -- & relation & 36.0 & 14.4 \\
\hline 4 & 04072700 & Neenah Ck nr Briggsville & $43^{\circ} 40^{\prime} 08^{\prime \prime}$ & $89^{\circ} 33^{\prime} 24^{\prime \prime}$ & 04081000 & 80.3 & .3690 & relation & 50.0 & 8.5 \\
\hline 5 & 04072706 & So Br Neenah Ck nr Briggsville & $43^{\circ} 38^{\prime} 53^{\prime \prime}$ & $89^{\circ} 36^{\prime} 00^{\prime \prime}$ & 04073500 & 28.77 & .8270 & equation & 25.3 & 11.9 \\
\hline 6 & 04072715 & Neenah Ck nr Endeavor & $43^{\circ} 38^{\prime} 41^{\prime \prime}$ & $89^{\circ} 28^{\prime} 07^{\prime \prime}$ & 04073500 & 157.4 & .6599 & equation & 127.3 & 11.0 \\
\hline 7 & 040727265 & Spring Ck nr Anacker & $43^{\circ} 36^{\prime} 21^{\prime \prime}$ & $89^{\circ} 23^{\prime} 12^{\prime \prime}$ & 04073500 & 17.4 & .1157 & equation & 5.4 & 4.2 \\
\hline 8 & 04072728 & French Ck nr Anacker & $43^{\circ} 37^{\prime} 42^{\prime \prime}$ & $89^{\circ} 24^{\prime} 31^{\prime \prime}$ & 04073500 & 24.35 & .1812 & equation & 9.7 & 5.4 \\
\hline 9 & 04072732 & Good Earth Ck nr Endeavor & $43^{\circ} 39^{\prime} 57^{\prime \prime}$ & $89^{\circ} 25^{\prime} 28^{\prime \prime}$ & 04073500 & 16.48 & .2368 & equation & 7.5 & 6.2 \\
\hline 10 & 04072743 & Fox R at Montello & $43^{\circ} 47^{\prime} 08^{\prime \prime}$ & $89^{\circ} 19^{\prime} 32^{\prime \prime}$ & 04073500 & 398.2 & .3679 & equation & 300.0 & 10.2 \\
\hline 11 & 04072790 & Wfield Ck nr Harrisville & $43^{\circ} 54^{\prime} 04^{\prime \prime}$ & $89^{\circ} 25^{\prime} 34^{\prime \prime}$ & 05401535 & 48 & 1.0525 & equation & 48.3 & 13.7 \\
\hline 12 & 04072942 & Grand R nr Fairwater & $43^{\circ} 43^{\prime} 53^{\prime \prime}$ & $88^{\circ} 53^{\prime} 10^{\prime \prime}$ & 04073050 & 24.6 & .0700 & relation & 4.5 & 2.5 \\
\hline 13 & 04073023 & Grand $\mathrm{R}$ at Markasan & $43^{\circ} 42^{\prime} 22^{\prime \prime}$ & $88^{\circ} 59^{\prime} 25^{\prime \prime}$ & 04073050 & 57.3 & .1147 & relation & 17.0 & 4.0 \\
\hline 14 & 04073100 & Belle Fountain Ck nr Kingston & $43^{\circ} 41^{\prime} 57^{\prime \prime}$ & $89^{\circ} 12^{\prime} 44^{\prime \prime}$ & 04073500 & 37.4 & .1550 & relation & 17.5 & 6.4 \\
\hline 15 & 04073180 & Mecan R nr Richford & $44^{\circ} 03^{\prime} 04^{\prime \prime}$ & $89^{\circ} 27^{\prime} 50^{\prime \prime}$ & 05401535 & 2.98 & 4.1499 & equation & 5.8 & ${ }^{2} 26.4$ \\
\hline 16 & 04073185 & Schmudlach Ck nr Richford & $44^{\circ} 03^{\prime} 17^{\prime \prime}$ & $89^{\circ} 27^{\prime} 03^{\prime \prime}$ & 05401535 & 13.1 & .0980 & equation & 3.7 & 3.9 \\
\hline 17 & 04073190 & Mecan R at Richford & $44^{\circ} 01^{\prime} 33^{\prime \prime}$ & $89^{\circ} 27^{\prime} 53^{\prime \prime}$ & 05401535 & 21.6 & 1.0965 & equation & 21.9 & 13.7 \\
\hline 18 & 04073207 & W Br Little Pine Ck nr Richford & $44^{\circ} 01^{\prime} 27^{\prime \prime}$ & $89^{\circ} 21^{\prime} 08^{\prime \prime}$ & 05401535 & 5.78 & .9951 & equation & 5.4 & 12.7 \\
\hline 19 & 04073220 & Little Pine Ck nr Richford & $43^{\circ} 59^{\prime} 18^{\prime \prime}$ & $89^{\circ} 20^{\prime} 31^{\prime \prime}$ & 05401535 & 11.4 & .8719 & equation & 10.1 & 12.0 \\
\hline 20 & 04073240 & Chaffee Ck nr Coloma & $43^{\circ} 59^{\prime} 01^{\prime \prime}$ & $89^{\circ} 29^{\prime} 51^{\prime \prime}$ & 05401535 & 4.28 & 2.5283 & equation & 6.5 & ${ }^{2} 20.5$ \\
\hline 21 & 04073260 & S Br Wedde Ck nr Richford & $44^{\circ} 00^{\prime} 10^{\prime \prime}$ & $89^{\circ} 27^{\prime} 13^{\prime \prime}$ & 05401535 & 16 & .5844 & equation & 11.6 & 9.8 \\
\hline 22 & 04073270 & S Br Wedde Ck nr Richford & $43^{\circ} 59^{\prime} 05^{\prime \prime}$ & $89^{\circ} 24^{\prime} 25^{\prime \prime}$ & 05401535 & 17.7 & .6869 & equation & 14.0 & 10.7 \\
\hline 23 & 04073290 & Wedde Ck nr Richford & $43^{\circ} 57^{\prime} 19^{\prime \prime}$ & $89^{\circ} 21^{\prime} 53^{\prime \prime}$ & 05401535 & 26 & .6249 & equation & 19.7 & 10.3 \\
\hline 24 & 04073300 & Chaffee Ck nr Neshkoro & $43^{\circ} 57^{\prime} 02^{\prime \prime}$ & $89^{\circ} 21^{\prime} 04^{\prime \prime}$ & 05401535 & 46.3 & .6900 & relation & 48.0 & 14.1 \\
\hline
\end{tabular}

${ }^{1}$ Low-flow discharge measurements at partial-record stations were compared to one or more nearby USGS gaging stations to establish a relation between measured discharge and concurrent daily discharge. The nearby gaging station that provided the best relation with the partial-record station was chosen as the index station and was associated with nearby miscellaneous measurement sites.

${ }^{2}$ Mismatch between surface-water and ground-water drainage area. 
[USGS, U.S. Geological Survey; ID, identification number; $\mathrm{dd}^{\circ} \mathrm{mm}$ 'ss", degrees, minutes, seconds; $\mathrm{mi}^{2}$, square mile; $\mathrm{ft}^{3} / \mathrm{s} / \mathrm{mi}^{2}$, cubic foot per second per square mile; $\mathrm{ft}^{3} / \mathrm{s}$, cubic foot per second; equation, statewide regression equation; relation, established relation line with index station; R, River; Bk, Brook; Br, Branch; Fk, Fork; Ck, Creek; Tr, Tributary; nr, near; N, North; S, South; E, East; W, West;

,-- not determined in historical low-flow reports. See references for full list of low-flow reports.]

\begin{tabular}{|c|c|c|c|c|c|c|c|c|c|c|}
\hline $\begin{array}{c}\text { Map } \\
\text { number }\end{array}$ & $\begin{array}{c}\text { USGS } \\
\text { station ID }\end{array}$ & Station name & $\begin{array}{c}\text { Latitude } \\
\left.\text { (dd }{ }^{\circ} \mathrm{mm} ' s s^{\prime \prime}\right)\end{array}$ & $\begin{array}{l}\text { Longitude } \\
\text { (dd'mm'ss") }\end{array}$ & $\begin{array}{l}\text { Index } \\
\text { station }{ }^{1}\end{array}$ & $\begin{array}{c}\text { Drainage } \\
\text { area } \\
\left(\mathrm{mi}^{2}\right)\end{array}$ & $\begin{array}{c}\text { Base flow } \\
\text { factor } \\
\left(\mathrm{ft}^{3} / \mathrm{s} / \mathrm{mi}^{2}\right)\end{array}$ & $\begin{array}{c}\text { Base flow } \\
\text { method }\end{array}$ & $\begin{array}{c}\text { Base flow } \\
\quad\left(\mathrm{ft}^{3} / \mathrm{s}\right)\end{array}$ & $\begin{array}{c}\text { Recharge } \\
\text { (inches) }\end{array}$ \\
\hline 25 & 04073310 & Mecan R nr Harrisville & $43^{\circ} 54^{\prime} 57^{\prime \prime}$ & $89^{\circ} 18^{\prime} 53^{\prime \prime}$ & 05401535 & 98 & 1.1019 & equation & 102.5 & 14.2 \\
\hline 26 & 04073318 & Mecan R nr Germania & $43^{\circ} 54^{\prime} 35^{\prime \prime}$ & $89^{\circ} 17^{\prime} 25^{\prime \prime}$ & 05401535 & 107 & .9990 & equation & 106.5 & 13.5 \\
\hline 27 & 04073330 & Mecan R nr Neshkoro & $43^{\circ} 53^{\prime} 29^{\prime \prime}$ & $89^{\circ} 15^{\prime} 13^{\prime \prime}$ & 05401535 & 122 & .8398 & equation & 111.2 & 12.4 \\
\hline 28 & 04073396 & White R at Wautoma & $44^{\circ} 03^{\prime} 54^{\prime \prime}$ & $89^{\circ} 17^{\prime} 26^{\prime \prime}$ & 05401535 & 13.7 & 1.3508 & equation & 21.0 & ${ }^{2} 20.8$ \\
\hline 29 & 04073400 & Bird Ck at Wautoma & $44^{\circ} 04^{\prime} 06^{\prime \prime}$ & $89^{\circ} 18^{\prime} 08^{\prime \prime}$ & 05401535 & 4.14 & 2.0000 & relation & 10.5 & 234.4 \\
\hline 30 & 04073420 & Lunch Ck nr Neshkoro & $43^{\circ} 57^{\prime} 35^{\prime \prime}$ & $89^{\circ} 16^{\prime} 30^{\prime \prime}$ & 05401050 & 18.3 & .4590 & relation & 14.5 & 10.8 \\
\hline 31 & 04073440 & White R nr Princeton & $43^{\circ} 55^{\prime} 03^{\prime \prime}$ & $89^{\circ} 05^{\prime} 01^{\prime \prime}$ & 04073500 & 131 & 1.0863 & equation & 136.7 & 14.2 \\
\hline 32 & 04073450 & Sucker Ck nr Berlin & $43^{\circ} 56^{\prime} 30^{\prime \prime}$ & $89^{\circ} 04^{\prime} 47^{\prime \prime}$ & 04073500 & 20.6 & .3380 & relation & 14.7 & 9.7 \\
\hline 33 & 04073464 & Silver Ck at Ripon & $43^{\circ} 51^{\prime} 14^{\prime \prime}$ & $88^{\circ} 51^{\prime} 36^{\prime \prime}$ & 04073050 & 26.7 & .2554 & relation & 7.8 & 4.0 \\
\hline 34 & 04073472 & Puchyan R nr Green Lk & $43^{\circ} 51^{\prime} 24^{\prime \prime}$ & $88^{\circ} 56^{\prime} 40^{\prime \prime}$ & 04073050 & 105 & .0683 & relation & 34.0 & 4.4 \\
\hline 35 & 04073900 & Eightmile Ck at Fisk & $43^{\circ} 57^{\prime} 20^{\prime \prime}$ & $88^{\circ} 40^{\prime} 44^{\prime \prime}$ & 04073500 & 22.9 & .0012 & equation & .7 & .4 \\
\hline 36 & 04074290 & Wolf R nr Nashville & $45^{\circ} 34^{\prime} 20^{\prime \prime}$ & $89^{\circ} 01^{\prime} 07^{\prime \prime}$ & 04074950 & 42.8 & .4190 & equation & 26.6 & 8.4 \\
\hline 37 & 04074350 & Wolf R nr Nashville & $45^{\circ} 31^{\prime} 55^{\prime \prime}$ & $89^{\circ} 06^{\prime} 43^{\prime \prime}$ & 04074950 & 60.77 & .3389 & equation & 34.1 & 7.6 \\
\hline 38 & 04074508 & Swamp Ck nr Crandon & $45^{\circ} 30^{\prime} 03^{\prime \prime}$ & $88^{\circ} 54^{\prime} 11 "$ & 04074950 & 24.2 & .2473 & equation & 11.3 & 6.3 \\
\hline 39 & 04074520 & Swamp Ck nr Crandon & $45^{\circ} 29^{\prime} 52^{\prime \prime}$ & $88^{\circ} 54^{\prime} 40^{\prime \prime}$ & 04074950 & 39.2 & .4057 & equation & 23.9 & 8.3 \\
\hline 40 & 04074542 & Gliske Ck nr Mole Lk & $45^{\circ} 30^{\prime} 42^{\prime \prime}$ & $88^{\circ} 58^{\prime} 02^{\prime \prime}$ & 04074950 & 2.43 & 1.2692 & equation & 2.5 & 14.2 \\
\hline 41 & 04074560 & Squaw Ck nr Post Lk & $45^{\circ} 29^{\prime} 02^{\prime \prime}$ & $89^{\circ} 01^{\prime} 32^{\prime \prime}$ & 04074950 & 9.15 & .7168 & equation & 7.3 & 10.8 \\
\hline 42 & 04074600 & Swamp Ck nr Post Lk & $45^{\circ} 26^{\prime} 27^{\prime \prime}$ & $89^{\circ} 02^{\prime} 27^{\prime \prime}$ & 04074950 & 76.5 & .8574 & equation & 69.9 & 12.4 \\
\hline 43 & 04074653 & Pickerel Ck nr Mole Lk & $45^{\circ} 26^{\prime} 37^{\prime \prime}$ & $88^{\circ} 56^{\prime} 11 "$ & 04074950 & 6.13 & .2010 & equation & 2.5 & 5.5 \\
\hline 44 & 04074654 & Pickerel Ck Tr nr Mole Lk & $45^{\circ} 26^{\prime} 46^{\prime \prime}$ & $88^{\circ} 57^{\prime} 26^{\prime \prime}$ & 04074950 & 2.16 & .2382 & equation & .9 & 5.9 \\
\hline 45 & 04074657 & Pickerel Ck nr Mole Lk & $45^{\circ} 25^{\prime} 16^{\prime \prime}$ & $88^{\circ} 56^{\prime} 08^{\prime \prime}$ & 04074950 & 14.3 & .3704 & equation & 8.2 & 7.8 \\
\hline 46 & 04074666 & Pickerel Ck nr Kole Lk & $45^{\circ} 23^{\prime} 31^{\prime \prime}$ & $88^{\circ} 58^{\prime} 20^{\prime \prime}$ & 04074950 & 78.43 & .2250 & equation & 35.7 & 6.2 \\
\hline 47 & 04074685 & Hunting $\mathrm{R}$ at Elcho & $45^{\circ} 26^{\prime} 20^{\prime \prime}$ & $89^{\circ} 12^{\prime} 18^{\prime \prime}$ & 04074950 & 3.39 & .4258 & equation & 2.0 & 8.1 \\
\hline 48 & 04074700 & Hunting R nr Elcho & $45^{\circ} 25^{\prime} 10^{\prime \prime}$ & $89^{\circ} 11^{\prime} 15^{\prime \prime}$ & 05394500 & 10.7 & .6010 & relation & 7.8 & 9.9 \\
\hline
\end{tabular}

${ }^{1}$ Low-flow discharge measurements at partial-record stations were compared to one or more nearby USGS gaging stations to establish a relation between measured discharge and concurrent daily discharge. The nearby gaging station that provided the best relation with the partial-record station was chosen as the index station and was associated with nearby miscellaneous measurement sites.

${ }^{2}$ Mismatch between surface-water and ground-water drainage area. 
Appendix 8D. Average annual base flow and corresponding recharge, 1970-99, for partial-record stations in the Fox-Wolf River Basin-Continued.

[USGS, U.S. Geological Survey; ID, identification number; $\mathrm{dd}^{\circ} \mathrm{mm}$ 'ss", degrees, minutes, seconds; $\mathrm{mi}^{2}$, square mile; $\mathrm{ft}^{3} / \mathrm{s} / \mathrm{mi}^{2}$, cubic foot per second per square mile; $\mathrm{ft}^{3} / \mathrm{s}$, cubic foot per second; equation, statewide regression equation; relation, established relation line with index station; R, River; Bk, Brook; Br, Branch; Fk, Fork; Ck, Creek; Tr, Tributary; nr, near; N, North; S, South; E, East; W, West; --, not determined in historical low-flow reports. See references for full list of low-flow reports.]

\begin{tabular}{|c|c|c|c|c|c|c|c|c|c|c|}
\hline $\begin{array}{c}\text { Map } \\
\text { number }\end{array}$ & $\begin{array}{c}\text { USGS } \\
\text { station ID }\end{array}$ & Station name & $\begin{array}{c}\text { Latitude } \\
\text { (dd }{ }^{\circ} \mathrm{mm} \text { 'ss") }\end{array}$ & $\begin{array}{c}\text { Longitude } \\
\text { (dd }{ }^{\circ} \mathrm{mm} \text { 'ss") }\end{array}$ & $\begin{array}{l}\text { Index } \\
\text { station }{ }^{1}\end{array}$ & $\begin{array}{c}\text { Drainage } \\
\text { area } \\
\left(\mathrm{mi}^{2}\right)\end{array}$ & $\begin{array}{c}\text { Base flow } \\
\text { factor } \\
\left(\mathrm{ft}^{3} / \mathrm{s} / \mathrm{mi}^{2}\right)\end{array}$ & $\begin{array}{l}\text { Base flow } \\
\text { method }\end{array}$ & $\begin{array}{c}\text { Base flow } \\
\quad\left(\mathrm{ft}^{3} / \mathrm{s}\right)\end{array}$ & $\begin{array}{c}\text { Recharge } \\
\text { (inches) }\end{array}$ \\
\hline 49 & 04074750 & Hunting $\mathrm{R}$ at Pearson & $45^{\circ} 22^{\prime} 17^{\prime \prime}$ & $89^{\circ} 01^{\prime} 56^{\prime \prime}$ & 04074950 & 35.4 & 1.0842 & equation & 36.0 & 13.8 \\
\hline 50 & 04074753 & Wolf R nr Pickerel & $45^{\circ} 20^{\prime} 56^{\prime \prime}$ & $88^{\circ} 59^{\prime} 39^{\prime \prime}$ & 04074950 & 298 & .4394 & equation & 197.5 & 9.0 \\
\hline 51 & 04074780 & Wolf R at Lily & $45^{\circ} 18^{\prime} 29^{\prime \prime}$ & $88^{\circ} 51^{\prime} 27^{\prime \prime}$ & 04074950 & 332 & .4191 & equation & 215.2 & 8.8 \\
\hline 52 & 04074850 & Lily R nr Lily & $45^{\circ} 20^{\prime} 59^{\prime \prime}$ & $88^{\circ} 49^{\prime} 52^{\prime \prime}$ & 04071000 & 45.6 & .5460 & relation & 39.0 & 11.6 \\
\hline 53 & 04074880 & Lily R at Lily & $45^{\circ} 18^{\prime} 27^{\prime \prime}$ & $88^{\circ} 51^{\prime} 14^{\prime \prime}$ & 04071000 & 70.2 & .4649 & equation & 46.5 & 9.0 \\
\hline 54 & 04074910 & Ninemile Ck at Hollister & $45^{\circ} 14^{\prime} 50^{\prime \prime}$ & $88^{\circ} 47^{\prime} 15^{\prime \prime}$ & 04071000 & 21.6 & .3856 & equation & 12.7 & 8.0 \\
\hline 55 & 04075300 & Elton Ck nr Langlade & $45^{\circ} 06^{\prime} 08^{\prime \prime}$ & $88^{\circ} 49^{\prime} 59^{\prime \prime}$ & 04074950 & 25.17 & .9077 & equation & 23.2 & 12.5 \\
\hline 56 & 04075350 & Evergreen R nr Langlade & $45^{\circ} 04^{\prime} 28^{\prime \prime}$ & $88^{\circ} 42^{\prime} 31^{\prime \prime}$ & 04074950 & 54.6 & .7786 & equation & 47.1 & 11.7 \\
\hline 57 & 04075803 & W Br Wolf R nr Neopit & $45^{\circ} 01^{\prime} 06^{\prime \prime}$ & $88^{\circ} 52^{\prime} 27^{\prime \prime}$ & 04078500 & 41.8 & .6728 & equation & 33.2 & 10.8 \\
\hline 58 & 04075807 & Little W Br Wolf R nr Zoar & $45^{\circ} 05^{\prime} 02^{\prime \prime}$ & $88^{\circ} 58^{\prime} 55^{\prime \prime}$ & 04074950 & 9.19 & .6219 & equation & 6.8 & 10.1 \\
\hline 59 & 04075809 & Rabe Ck nr Polar & $45^{\circ} 08^{\prime} 23^{\prime \prime}$ & $88^{\circ} 59^{\prime} 20^{\prime \prime}$ & 04074950 & 7.2 & .4648 & equation & 4.6 & 8.6 \\
\hline 60 & 04075850 & Little W Br Wolf R nr Neopit & $44^{\circ} 59^{\prime} 11 "$ & $88^{\circ} 52^{\prime} 49^{\prime \prime}$ & 04078500 & 45.84 & .4039 & equation & 28.0 & 8.3 \\
\hline 61 & 04075905 & W Branch Red R nr Phlox & $45^{\circ} 03^{\prime} 42^{\prime \prime}$ & $89^{\circ} 06^{\prime} 08^{\prime \prime}$ & 04078500 & 5.11 & .8422 & equation & 4.4 & 11.6 \\
\hline 62 & 04076080 & W Br Wolf R at Neopit & $44^{\circ} 58^{\prime} 54^{\prime \prime}$ & $88^{\circ} 49^{\prime} 01^{\prime \prime}$ & 04078500 & 98.6 & .5650 & relation & 115.0 & 15.8 \\
\hline 63 & 04076400 & Little W Br Ck nr Neopit & $45^{\circ} 00^{\prime} 54^{\prime \prime}$ & $88^{\circ} 46^{\prime} 11^{\prime \prime}$ & 04078500 & 17 & .4190 & relation & 17.5 & 14.0 \\
\hline 64 & 04077150 & Wolf $\mathrm{R} \operatorname{Tr} \# 1$ at Keshena & $44^{\circ} 53^{\prime} 03^{\prime \prime}$ & $88^{\circ} 37^{\prime} 41^{\prime \prime}$ & 04071000 & 6.6 & .4758 & equation & 4.2 & 8.7 \\
\hline 65 & 04077550 & Red R nr Antigo & $45^{\circ} 04^{\prime} 22^{\prime \prime}$ & $89^{\circ} 00^{\prime} 44^{\prime \prime}$ & 05397500 & 20.6 & 2.5900 & relation & 41.5 & 227.4 \\
\hline 66 & 04077595 & W Branch Red R at Mattoon & $45^{\circ} 00^{\prime} 44^{\prime \prime}$ & $89^{\circ} 02^{\prime} 03^{\prime \prime}$ & 04078500 & 22 & .4580 & relation & 18.5 & 11.4 \\
\hline 67 & 04077670 & Miller Ck nr Neopit & $44^{\circ} 56^{\prime} 34^{\prime \prime}$ & $88^{\circ} 49^{\prime} 55^{\prime \prime}$ & 04078500 & 10.9 & .7656 & equation & 9.0 & 11.2 \\
\hline 68 & 04077675 & Red $\mathrm{R}$ at Gresham & $44^{\circ} 50^{\prime} 28^{\prime \prime}$ & $88^{\circ} 45^{\prime} 40^{\prime \prime}$ & 04078500 & 159 & .5862 & equation & 120.9 & 10.3 \\
\hline 69 & 04077700 & Red $\mathrm{R}$ at Thornton & $44^{\circ} 48^{\prime} 10^{\prime \prime}$ & $88^{\circ} 40^{\prime} 24^{\prime \prime}$ & 04071000 & 195 & .5566 & equation & 144.9 & 10.1 \\
\hline 70 & 04077800 & Pickerel $\mathrm{Ck}$ at Cecil & $44^{\circ} 48^{\prime} 32^{\prime \prime}$ & $88^{\circ} 27^{\prime} 02^{\prime \prime}$ & 04071000 & 13.2 & .0653 & relation & 2.0 & 2.0 \\
\hline 71 & 04077820 & Duchess Ck nr Cecil & $44^{\circ} 49^{\prime} 53^{\prime \prime}$ & $88^{\circ} 28^{\prime} 02^{\prime \prime}$ & 04071000 & 8.34 & .0860 & relation & 1.6 & 2.6 \\
\hline 72 & 04077830 & Loon Lk nr Cecil & $44^{\circ} 49^{\prime} 51 "$ & $88^{\circ} 30^{\prime} 06^{\prime \prime}$ & 04071000 & 15.6 & .4291 & equation & 9.6 & 8.4 \\
\hline
\end{tabular}

${ }^{1}$ Low-flow discharge measurements at partial-record stations were compared to one or more nearby USGS gaging stations to establish a relation between measured discharge and concurrent daily discharge. The nearby gaging station that provided the best relation with the partial-record station was chosen as the index station and was associated with nearby miscellaneous measurement sites.

${ }^{2}$ Mismatch between surface-water and ground-water drainage area. 
[USGS, U.S. Geological Survey; ID, identification number; $\mathrm{dd}^{\circ} \mathrm{mm}$ 'ss", degrees, minutes, seconds; $\mathrm{mi}^{2}$, square mile; $\mathrm{ft}^{3} / \mathrm{s} / \mathrm{mi}^{2}$, cubic foot per second per square mile; $\mathrm{ft}^{3} / \mathrm{s}$, cubic foot per second; equation, statewide regression equation; relation, established relation line with index station; R, River; Bk, Brook; Br, Branch; Fk, Fork; Ck, Creek; Tr, Tributary; nr, near; N, North; S, South; E, East; W, West;

,-- not determined in historical low-flow reports. See references for full list of low-flow reports.]

\begin{tabular}{|c|c|c|c|c|c|c|c|c|c|c|}
\hline $\begin{array}{c}\text { Map } \\
\text { number }\end{array}$ & $\begin{array}{c}\text { USGS } \\
\text { station ID }\end{array}$ & Station name & $\begin{array}{c}\text { Latitude } \\
\text { (dd }{ }^{\circ} \mathrm{mm} \text { 'ss") }\end{array}$ & $\begin{array}{c}\text { Longitude } \\
\text { (ddºmm'ss") }\end{array}$ & $\begin{array}{l}\text { Index } \\
\text { station }^{1}\end{array}$ & $\begin{array}{c}\text { Drainage } \\
\text { area } \\
\left(\mathrm{mi}^{2}\right)\end{array}$ & $\begin{array}{c}\text { Base flow } \\
\text { factor } \\
\left(\mathrm{ft}^{3} / \mathrm{s} / \mathrm{mi}^{2}\right)\end{array}$ & $\begin{array}{l}\text { Base flow } \\
\text { method }\end{array}$ & $\begin{array}{c}\text { Base flow } \\
\left(\mathrm{ft}^{3} / \mathrm{s}\right)\end{array}$ & $\begin{array}{c}\text { Recharge } \\
\text { (inches) }\end{array}$ \\
\hline 73 & 04077871 & Shawano Lk Outlet nr Shawano & $44^{\circ} 47^{\prime} 29^{\prime \prime}$ & $88^{\circ} 34^{\prime} 07^{\prime \prime}$ & 04071000 & 71.2 & 0.7051 & equation & 58.6 & 11.2 \\
\hline 74 & 04078005 & Wolf R Tr nr Shawano & $44^{\circ} 43^{\prime} 45^{\prime \prime}$ & $88^{\circ} 33^{\prime} 30^{\prime \prime}$ & 04071000 & 12.7 & .0318 & equation & 2.0 & 2.2 \\
\hline 75 & 04078010 & Wolf R Tr nr Belle Plaine & $44^{\circ} 40^{\prime} 29^{\prime \prime}$ & $88^{\circ} 35^{\prime} 43^{\prime \prime}$ & 04071000 & 14.8 & .1118 & equation & 4.5 & 4.2 \\
\hline 76 & 04078022 & W Br Shioc R at Bonduel & $44^{\circ} 44^{\prime} 04^{\prime \prime}$ & $88^{\circ} 27^{\prime} 06^{\prime \prime}$ & 04071000 & 5.44 & .0720 & relation & 1.2 & 3.0 \\
\hline 77 & 04078028 & W Br Shioc R nr Bonduel & $44^{\circ} 41^{\prime} 31^{\prime \prime}$ & $88^{\circ} 28^{\prime} 44^{\prime \prime}$ & 04071000 & 16.5 & .1151 & equation & 5.1 & 4.2 \\
\hline 78 & 04078036 & E Br Shioc R nr Navarino & $44^{\circ} 38^{\prime} 43^{\prime \prime}$ & $88^{\circ} 29^{\prime} 05^{\prime \prime}$ & 04071000 & 16.5 & .0190 & equation & 2.0 & 1.7 \\
\hline 79 & 04078039 & Mink Ck nr Navarino & $44^{\circ} 37^{\prime} 36^{\prime \prime}$ & $88^{\circ} 29^{\prime} 11^{\prime \prime}$ & 04071000 & 7.58 & .0057 & equation & .5 & .9 \\
\hline 80 & 04078050 & Shioc R at Nichols & $44^{\circ} 33^{\prime} 54^{\prime \prime}$ & $88^{\circ} 28^{\prime} 58^{\prime \prime}$ & 04071000 & 92.2 & .0088 & relation & 10.0 & 1.5 \\
\hline 81 & 04078054 & Black Ck at Seymore & $44^{\circ} 30^{\prime} 55^{\prime \prime}$ & $88^{\circ} 20^{\prime} 41^{\prime \prime}$ & 04071000 & 22.9 & .0050 & relation & .2 & .1 \\
\hline 82 & 04078055 & Black Ck at Black Ck & $44^{\circ} 28^{\prime} 55^{\prime \prime}$ & $88^{\circ} 27^{\prime} 03^{\prime \prime}$ & 04071000 & 44.7 & .0150 & relation & 3.2 & 1.0 \\
\hline 83 & 04078057 & Shioc R nr Shiocton & $44^{\circ} 29^{\prime} 30^{\prime \prime}$ & $88^{\circ} 31^{\prime} 41^{\prime \prime}$ & 04071000 & 184 & .0288 & equation & 29.3 & 2.2 \\
\hline 84 & 04078080 & Bear Ck at Stephensville & $44^{\circ} 22^{\prime} 15^{\prime \prime}$ & $88^{\circ} 35^{\prime} 05^{\prime \prime}$ & 04079000 & 62.5 & .0230 & relation & 3.5 & .8 \\
\hline 85 & 04078085 & Black Otter Ck at Hortonville & $44^{\circ} 20^{\prime} 03^{\prime \prime}$ & $88^{\circ} 38^{\prime} 42^{\prime \prime}$ & 04081000 & 15.9 & .0040 & relation & 1.2 & 1.0 \\
\hline 86 & 04078092 & M Br Embarrass R nr Eland & $44^{\circ} 52^{\prime} 00^{\prime \prime}$ & $89^{\circ} 10^{\prime} 05^{\prime \prime}$ & 04078500 & 66.5 & .5898 & equation & 49.8 & 10.2 \\
\hline 87 & 04078098 & Railroad Ck nr Birnamwood & $44^{\circ} 54^{\prime} 11^{\prime \prime}$ & $89^{\circ} 12^{\prime} 36^{\prime \prime}$ & 04078500 & 2.87 & .2470 & equation & 1.5 & 6.9 \\
\hline 88 & 04078100 & S Br Embarrass R nr Wittenberg & $44^{\circ} 49^{\prime} 37^{\prime \prime}$ & $89^{\circ} 13^{\prime} 20^{\prime \prime}$ & 04078500 & 26.4 & .2860 & relation & 15.0 & 7.7 \\
\hline 89 & 04078109 & S Br Embarrass R nr Wittenberg & $44^{\circ} 47^{\prime} 07^{\prime \prime}$ & $89^{\circ} 11^{\prime} 03^{\prime \prime}$ & 04078500 & 50.5 & .2646 & equation & 24.8 & 6.7 \\
\hline 90 & 04078190 & S Br Embarrass R at Tigerton & $44^{\circ} 44^{\prime} 30^{\prime \prime}$ & $89^{\circ} 03^{\prime} 25^{\prime \prime}$ & 04078500 & 90.5 & .3270 & relation & 57.0 & 8.6 \\
\hline 91 & 04078298 & Embarrass $\mathrm{R}$ at Caroline & $44^{\circ} 43^{\prime} 23^{\prime \prime}$ & $88^{\circ} 52^{\prime} 31^{\prime \prime}$ & 04078500 & 239 & .3020 & relation & 135.0 & 7.7 \\
\hline 92 & 04078300 & Embarrass $\mathrm{R}$ at Caroline & $44^{\circ} 43^{\prime} 25^{\prime \prime}$ & $88^{\circ} 52^{\prime} 26^{\prime \prime}$ & 04078500 & 239 & .3160 & equation & 132.9 & 7.6 \\
\hline 93 & 04078490 & Mill Ck nr Pella & $44^{\circ} 44^{\prime} 40^{\prime \prime}$ & $88^{\circ} 44^{\prime} 48^{\prime \prime}$ & 04078500 & 40.6 & .2060 & relation & 15.5 & 5.2 \\
\hline 94 & 04078600 & N Br Pigeon R nr Marion & $44^{\circ} 40^{\prime} 21^{\prime \prime}$ & $88^{\circ} 56^{\prime} 46^{\prime \prime}$ & 04078500 & 10.8 & .2040 & relation & 5.3 & 6.7 \\
\hline 95 & 04078605 & $\mathrm{~N}$ Br Pigeon $\mathrm{R}$ at Marion & $44^{\circ} 40^{\prime} 29^{\prime \prime}$ & $88^{\circ} 52^{\prime} 42^{\prime \prime}$ & 04078500 & 19.6 & .3030 & relation & 12.0 & 8.3 \\
\hline 96 & 04078650 & Pigeon $\mathrm{R}$ at Clintonville & $44^{\circ} 37^{\prime} 20^{\prime \prime}$ & $88^{\circ} 45^{\prime} 40^{\prime \prime}$ & 04078500 & 109 & .2916 & relation & 64.0 & 8.0 \\
\hline
\end{tabular}

${ }^{1}$ Low-flow discharge measurements at partial-record stations were compared to one or more nearby USGS gaging stations to establish a relation between measured discharge and concurrent daily discharge. The nearby gaging station that provided the best relation with the partial-record station was chosen as the index station and was associated with nearby miscellaneous measurement sites.

${ }^{2}$ Mismatch between surface-water and ground-water drainage area. 
Appendix 8D. Average annual base flow and corresponding recharge, 1970-99, for partial-record stations in the Fox-Wolf River Basin-Continued.

[USGS, U.S. Geological Survey; ID, identification number; $\mathrm{dd}^{\circ} \mathrm{mm}$ 'ss", degrees, minutes, seconds; $\mathrm{mi}^{2}$, square mile; $\mathrm{ft}^{3} / \mathrm{s} / \mathrm{mi}^{2}$, cubic foot per second per square mile; $\mathrm{ft}^{3} / \mathrm{s}$, cubic foot per second; equation, statewide regression equation; relation, established relation line with index station; R, River; Bk, Brook; Br, Branch; Fk, Fork; Ck, Creek; Tr, Tributary; nr, near; N, North; S, South; E, East; W, West; ,-- not determined in historical low-flow reports. See references for full list of low-flow reports.]

\begin{tabular}{|c|c|c|c|c|c|c|c|c|c|c|}
\hline $\begin{array}{c}\text { Map } \\
\text { number }\end{array}$ & $\begin{array}{c}\text { USGS } \\
\text { station ID }\end{array}$ & Station name & $\begin{array}{c}\text { Latitude } \\
\text { (dd }{ }^{\circ} \mathrm{mm} \text { 'ss") }\end{array}$ & $\begin{array}{c}\text { Longitude } \\
\text { (ddºmm'ss") }\end{array}$ & $\begin{array}{l}\text { Index } \\
\text { station } 1\end{array}$ & $\begin{array}{c}\text { Drainage } \\
\text { area } \\
\left(\mathrm{mi}^{2}\right)\end{array}$ & $\begin{array}{c}\text { Base flow } \\
\text { factor } \\
\left(\mathrm{ft}^{3} / \mathrm{s} / \mathrm{mi}^{2}\right)\end{array}$ & $\begin{array}{l}\text { Base flow } \\
\text { method }\end{array}$ & $\begin{array}{c}\text { Base flow } \\
\quad\left(\mathrm{ft}^{3} / \mathrm{s}\right)\end{array}$ & $\begin{array}{c}\text { Recharge } \\
\text { (inches) }\end{array}$ \\
\hline 97 & 04078780 & Bear Ck nr Bear Ck & $44^{\circ} 32^{\prime} 44^{\prime \prime}$ & $88^{\circ} 45^{\prime} 08^{\prime \prime}$ & 04078500 & 14.2 & 0.0178 & equation & 1.7 & 1.6 \\
\hline 98 & 04078785 & Bear Ck at Bear Ck & $44^{\circ} 31^{\prime} 54^{\prime \prime}$ & $88^{\circ} 42^{\prime} 37^{\prime \prime}$ & 04078500 & 19.7 & .0210 & relation & 2.0 & 1.4 \\
\hline 99 & 04078800 & Bear Ck nr Sugar Bush & $44^{\circ} 29^{\prime} 21^{\prime \prime}$ & $88^{\circ} 41^{\prime} 18^{\prime \prime}$ & 04078500 & 30.6 & .0110 & relation & .8 & .4 \\
\hline 100 & 04079600 & Little Wolf $\mathrm{R}$ at Galloway & $44^{\circ} 42^{\prime} 50^{\prime \prime}$ & $89^{\circ} 15^{\prime} 47^{\prime \prime}$ & 04078500 & 21.3 & .2870 & relation & 12.8 & 8.2 \\
\hline 101 & 04079603 & Holt Ck nr Galloway & $44^{\circ} 42^{\prime} 24^{\prime \prime}$ & $89^{\circ} 17^{\prime} 53^{\prime \prime}$ & 04078500 & 8.18 & .1760 & equation & 3.1 & 5.2 \\
\hline 102 & 04079607 & Holt Ck nr Galloway & $44^{\circ} 40^{\prime} 54^{\prime \prime}$ & $89^{\circ} 15^{\prime} 57^{\prime \prime}$ & 04078500 & 16.7 & .2321 & equation & 7.5 & 6.1 \\
\hline 103 & 04079630 & Flume Ck nr Rosholt & $44^{\circ} 37^{\prime} 40^{\prime \prime}$ & $89^{\circ} 18^{\prime} 16^{\prime \prime}$ & 04081000 & 19.2 & .1870 & relation & 4.6 & 3.3 \\
\hline 104 & 04079650 & Comet Ck nr Big Falls & $44^{\circ} 38^{\prime} 46^{\prime \prime}$ & $89^{\circ} 06^{\prime} 44^{\prime \prime}$ & 04078500 & 40.2 & .3280 & relation & 26.0 & 8.8 \\
\hline 105 & 04079657 & Comet Ck nr Big Falls & $44^{\circ} 37^{\prime} 15^{\prime \prime}$ & $89^{\circ} 07^{\prime} 18^{\prime \prime}$ & 04078500 & 42.6 & .3433 & equation & 23.9 & 7.6 \\
\hline 106 & 04079800 & Whitcomb Ck nr Big Falls & $44^{\circ} 34^{\prime} 05^{\prime \prime}$ & $88^{\circ} 56^{\prime} 07^{\prime \prime}$ & 04078500 & 27.4 & .3900 & relation & 15.5 & 7.7 \\
\hline 107 & 04079840 & Blake Ck at Symco & $44^{\circ} 30^{\prime} 16^{\prime \prime}$ & $88^{\circ} 54^{\prime} 36^{\prime \prime}$ & 04078500 & 30.1 & .2522 & equation & 14.3 & 6.4 \\
\hline 108 & 04079845 & Little Wolf $\mathrm{R}$ at Manawa & $44^{\circ} 27^{\prime} 57^{\prime \prime}$ & $88^{\circ} 55^{\prime} 19^{\prime \prime}$ & 04078500 & 311 & .3410 & relation & 205.0 & 9.0 \\
\hline 109 & 04079880 & S Br Little Wolf $\mathrm{R}$ at Iola & $44^{\circ} 30^{\prime} 33^{\prime \prime}$ & $89^{\circ} 08^{\prime} 16^{\prime \prime}$ & 04081000 & 30 & .4140 & relation & 17.0 & 7.7 \\
\hline 110 & 04079892 & S Br Little Wolf R at Scandinavia & $44^{\circ} 27^{\prime} 50^{\prime \prime}$ & $89^{\circ} 09^{\prime} 08^{\prime \prime}$ & 04081000 & 50.9 & .4050 & relation & 24.5 & 6.5 \\
\hline 111 & 04079900 & Peterson Ck nr Scandinavia & $44^{\circ} 26^{\prime} 03^{\prime \prime}$ & $89^{\circ} 09^{\prime} 48^{\prime \prime}$ & 04081000 & 25.6 & .4760 & relation & 20.0 & 10.6 \\
\hline 112 & 04080800 & Tomorrow R nr Nelsonville & $44^{\circ} 30^{\prime} 51^{\prime \prime}$ & $89^{\circ} 20^{\prime} 02^{\prime \prime}$ & 04081000 & 46 & .3520 & relation & 25.0 & 7.4 \\
\hline 113 & 04080895 & Tomorrow $\mathrm{R}$ at Amherst & $44^{\circ} 26^{\prime} 49^{\prime \prime}$ & $89^{\circ} 17^{\prime} 05^{\prime \prime}$ & 04081000 & 80.1 & .3060 & relation & 34.0 & 5.8 \\
\hline 114 & 04081100 & Walla Walla Ck nr Weyauwega & $44^{\circ} 17^{\prime} 20^{\prime \prime}$ & $88^{\circ} 55^{\prime} 53^{\prime \prime}$ & 04081000 & 52.4 & .3050 & relation & 24.0 & 6.2 \\
\hline 115 & 04081400 & Alder Ck nr Fremont & $44^{\circ} 11^{\prime} 04^{\prime \prime}$ & $88^{\circ} 50^{\prime} 44^{\prime \prime}$ & 04081000 & 14.2 & .0017 & relation & .3 & .3 \\
\hline 116 & 04081408 & Humphrey Ck nr Wild Rose & $44^{\circ} 11^{\prime} 05^{\prime \prime}$ & $89^{\circ} 14^{\prime} 45^{\prime \prime}$ & 04081000 & 20.6 & .3120 & relation & 12.5 & 8.2 \\
\hline 117 & 04081425 & Pine R at Poy Sippi & $44^{\circ} 08^{\prime} 09^{\prime \prime}$ & $88^{\circ} 59^{\prime} 46^{\prime \prime}$ & 04081000 & 99.4 & .6730 & relation & 93.0 & 12.7 \\
\hline 118 & 04081440 & Rattlesnake Ck nr Mt Morris & $44^{\circ} 06^{\prime} 45^{\prime \prime}$ & $89^{\circ} 11^{\prime} 32^{\prime \prime}$ & 04081000 & 20.4 & .5900 & relation & 17.5 & 11.7 \\
\hline 119 & 04081450 & Willow Ck nr Redgranite & $44^{\circ} 03^{\prime} 19^{\prime \prime}$ & $89^{\circ} 08^{\prime} 32^{\prime \prime}$ & 04081000 & 34.8 & .6790 & relation & 42.0 & 16.4 \\
\hline 120 & 04081456 & Willow Ck at Redgranite & $44^{\circ} 02^{\prime} 58^{\prime \prime}$ & $89^{\circ} 06^{\prime} 11^{\prime \prime}$ & 04081000 & 48.8 & 1.2000 & relation & 61.0 & ${ }^{2} 17.0$ \\
\hline
\end{tabular}

${ }^{1}$ Low-flow discharge measurements at partial-record stations were compared to one or more nearby USGS gaging stations to establish a relation between measured discharge and concurrent daily discharge. The nearby gaging station that provided the best relation with the partial-record station was chosen as the index station and was associated with nearby miscellaneous measurement sites.

${ }^{2}$ Mismatch between surface-water and ground-water drainage area. 
Appendix 8D. Average annual base flow and corresponding recharge, 1970-99, for partial-record stations in the Fox-Wolf River Basin-Continued.

[USGS, U.S. Geological Survey; ID, identification number; $\mathrm{dd}^{\circ} \mathrm{mm}$ 'ss", degrees, minutes, seconds; $\mathrm{mi}^{2}$, square mile; $\mathrm{ft}^{3} / \mathrm{s} / \mathrm{mi}^{2}$, cubic foot per second per square mile; $\mathrm{ft}^{3} / \mathrm{s}$, cubic foot per second; equation, statewide regression equation; relation, established relation line with index station; R, River; Bk, Brook; Br, Branch; Fk, Fork; Ck, Creek; Tr, Tributary; nr, near; N, North; S, South; E, East; W, West; ,-- not determined in historical low-flow reports. See references for full list of low-flow reports.]

\begin{tabular}{|c|c|c|c|c|c|c|c|c|c|c|}
\hline $\begin{array}{l}\text { Map } \\
\text { number }\end{array}$ & $\begin{array}{l}\text { USGS } \\
\text { station ID }\end{array}$ & Station name & $\begin{array}{c}\text { Latitude } \\
\left.\text { (dd }{ }^{\circ} \mathrm{mm} ' s s^{\prime \prime}\right)\end{array}$ & $\begin{array}{l}\text { Longitude } \\
\left.\text { (dd }{ }^{\circ} \mathrm{mm} ' s s^{\prime \prime}\right)\end{array}$ & $\begin{array}{l}\text { Index } \\
\text { station }\end{array}$ & $\begin{array}{c}\text { Drainage } \\
\text { area } \\
\left(\mathrm{mi}^{2}\right)\end{array}$ & $\begin{array}{c}\text { Base flow } \\
\text { factor } \\
\left(\mathrm{ft}^{3} / \mathrm{s} / \mathrm{mi}^{2}\right)\end{array}$ & $\begin{array}{l}\text { Base flow } \\
\text { method }\end{array}$ & $\begin{array}{l}\text { Base flow } \\
\qquad\left(\mathrm{ft}^{3} / \mathrm{s}\right)\end{array}$ & $\begin{array}{c}\text { Recharge } \\
\text { (inches) }\end{array}$ \\
\hline 121 & 04081800 & Daggets Ck nr Butte Des Morts & $44^{\circ} 05^{\prime} 48^{\prime \prime}$ & $88^{\circ} 37^{\prime} 16^{\prime \prime}$ & 04073500 & 10.6 & 0.0001 & equation & 0.1 & 0.1 \\
\hline 122 & 04082650 & Anderson Ck nr N Fond du Lac & $43^{\circ} 49^{\prime} 53^{\prime \prime}$ & $88^{\circ} 28^{\prime} 00^{\prime \prime}$ & 04073500 & 8.01 & .0048 & equation & .5 & .8 \\
\hline 123 & 04082813 & W Br Fond du Lac R at Rosendale & $43^{\circ} 48^{\prime} 54^{\prime \prime}$ & $88^{\circ} 39^{\prime} 00^{\prime \prime}$ & 04073500 & 5.2 & .1193 & equation & 1.6 & 4.2 \\
\hline 124 & 04082817 & W Br Fond du Lac Tr at Rosendale & $43^{\circ} 49^{\prime} 10^{\prime \prime}$ & $88^{\circ} 40^{\prime} 00^{\prime \prime}$ & 04073500 & 2.85 & .1480 & equation & 1.0 & 4.7 \\
\hline 125 & 04082826 & W Br Fond du Lac R Tr nr Rosendale & $43^{\circ} 50^{\prime} 06^{\prime \prime}$ & $88^{\circ} 43^{\prime} 00^{\prime \prime}$ & 04073500 & 12.2 & .0514 & equation & 2.5 & 2.8 \\
\hline 126 & 04082831 & Tr of Fond du Lac R nr Rosendale & $43^{\circ} 52^{\prime} 49^{\prime \prime}$ & $88^{\circ} 42^{\prime} 00^{\prime \prime}$ & 04073500 & 5.59 & .0099 & equation & .5 & 1.2 \\
\hline 127 & 04082841 & W Br Fond Du Lac R Tr nr Rosendale & $43^{\circ} 51^{\prime} 45^{\prime \prime}$ & $88^{\circ} 41^{\prime} 00^{\prime \prime}$ & 04073500 & 2.58 & .0204 & equation & .3 & 1.7 \\
\hline 128 & 04082866 & W Br Fond du Lac R Tr nr Rosendale & $43^{\circ} 48^{\prime} 54^{\prime \prime}$ & $88^{\circ} 38^{\prime} 00^{\prime \prime}$ & 04073500 & 7.77 & .0970 & equation & 2.2 & 3.8 \\
\hline 129 & 04082871 & W Br Fond du Lac R nr Rosendale & $43^{\circ} 49^{\prime} 31^{\prime \prime}$ & $88^{\circ} 36^{\prime} 00^{\prime \prime}$ & 04073500 & 55.2 & .0701 & equation & 13.6 & 3.4 \\
\hline 130 & 04082916 & W Br Fond du Lac R nr Rosendale & $43^{\circ} 45^{\prime} 58^{\prime \prime}$ & $88^{\circ} 31^{\prime} 00^{\prime \prime}$ & 04073500 & 78.5 & .0536 & equation & 17.0 & 2.9 \\
\hline 131 & 04083025 & E Br Fond du Lac R nr Fond du Lac & $43^{\circ} 42^{\prime} 08^{\prime \prime}$ & $88^{\circ} 36^{\prime} 00^{\prime \prime}$ & 04073500 & 4.71 & .0027 & equation & .2 & .6 \\
\hline 132 & 04083160 & Campground Ck nr Oakfield & $43^{\circ} 41^{\prime} 24^{\prime \prime}$ & $88^{\circ} 33^{\prime} 10^{\prime \prime}$ & 04073050 & 9.57 & .4980 & equation & 6.3 & 9.0 \\
\hline 133 & 04083295 & E Br Fond du Lac R nr Fond du Lac & $43^{\circ} 44^{\prime} 52^{\prime \prime}$ & $88^{\circ} 30^{\prime} 00^{\prime \prime}$ & 04073500 & 53.1 & .0630 & equation & 12.4 & 3.2 \\
\hline 134 & 04083425 & Parsons Ck nr Fond du Lac & $43^{\circ} 41^{\prime} 50^{\prime \prime}$ & $88^{\circ} 28^{\prime} 31^{\prime \prime}$ & 04073500 & 5.7 & .1267 & equation & 1.8 & 4.4 \\
\hline 135 & 04083435 & $\begin{array}{l}\text { E Br Fond du Lac R Tr nr Fond du } \\
\text { Lac }\end{array}$ & $43^{\circ} 42^{\prime} 45^{\prime \prime}$ & $88^{\circ} 28^{\prime} 00^{\prime \prime}$ & 04073500 & 5.87 & .1469 & equation & 2.0 & 4.7 \\
\hline 136 & 04083455 & E Br Fond du Lac R nr Fond du Lac & $43^{\circ} 43^{\prime} 24^{\prime \prime}$ & $88^{\circ} 28^{\prime} 00^{\prime \prime}$ & 04073500 & 73.7 & .0466 & equation & 14.8 & 2.7 \\
\hline 137 & 04083900 & De Neveu Ck nr Fond du Lac & $43^{\circ} 42^{\prime} 21^{\prime \prime}$ & $88^{\circ} 25^{\prime} 00^{\prime \prime}$ & 04073500 & 2.65 & .0136 & equation & .3 & 1.3 \\
\hline 138 & 04083935 & De Neveu Ck at Eden & $43^{\circ} 41^{\prime} 55^{\prime \prime}$ & $88^{\circ} 21^{\prime} 00^{\prime \prime}$ & 04073500 & 2.82 & .0863 & equation & .7 & 3.5 \\
\hline 139 & 04084020 & De Neveu Ck nr Fond du Lac & $43^{\circ} 44^{\prime} 46^{\prime \prime}$ & $88^{\circ} 24^{\prime} 00^{\prime \prime}$ & 04073500 & 16.4 & .0460 & equation & 3.2 & 2.6 \\
\hline 140 & 04084124 & Taycheedah Ck nr Fond du Lac & $43^{\circ} 47^{\prime} 02^{\prime \prime}$ & $88^{\circ} 24^{\prime} 00^{\prime \prime}$ & 04073050 & 14.1 & .1130 & equation & 4.3 & 4.2 \\
\hline 141 & 04081475 & Willow Ck nr Auroraville & $44^{\circ} 06^{\prime} 01^{\prime \prime}$ & $88^{\circ} 03^{\prime} 00^{\prime \prime}$ & 04086000 & 114 & .3897 & equation & 69.6 & 8.3 \\
\hline 142 & 04084200 & Lk Winnebago $\operatorname{Tr}$ at Brothertown & $43^{\circ} 58^{\prime} 17^{\prime \prime}$ & $88^{\circ} 18^{\prime} 35^{\prime \prime}$ & 04073500 & 5.1 & .0380 & equation & .9 & 2.3 \\
\hline 143 & 04084940 & Plum Ck nr Wrightstown & $44^{\circ} 19^{\prime} 02^{\prime \prime}$ & $88^{\circ} 10^{\prime} 20^{\prime \prime}$ & 04085200 & 35 & .1225 & equation & 11.4 & 4.4 \\
\hline
\end{tabular}

${ }^{1}$ Low-flow discharge measurements at partial-record stations were compared to one or more nearby USGS gaging stations to establish a relation between measured discharge and concurrent daily discharge. The nearby gaging station that provided the best relation with the partial-record station was chosen as the index station and was associated with nearby miscellaneous measurement sites.

${ }^{2}$ Mismatch between surface-water and ground-water drainage area. 
Appendix 8D. Average annual base flow and corresponding recharge, 1970-99, for partial-record stations in the Fox-Wolf River Basin-Continued.

[USGS, U.S. Geological Survey; ID, identification number; $\mathrm{dd}^{\circ} \mathrm{mm}$ 'ss", degrees, minutes, seconds; $\mathrm{mi}^{2}$, square mile; $\mathrm{ft}^{3} / \mathrm{s} / \mathrm{mi}^{2}$, cubic foot per second per square mile; $\mathrm{ft}^{3} / \mathrm{s}$, cubic foot per second; equation, statewide regression equation; relation, established relation line with index station; R, River; Bk, Brook; Br, Branch; Fk, Fork; Ck, Creek; Tr, Tributary; nr, near; N, North; S, South; E, East; W, West; --, not determined in historical low-flow reports. See references for full list of low-flow reports.]

\begin{tabular}{|c|c|c|c|c|c|c|c|c|c|c|}
\hline $\begin{array}{c}\text { Map } \\
\text { number }\end{array}$ & $\begin{array}{c}\text { USGS } \\
\text { station ID }\end{array}$ & Station name & $\begin{array}{c}\text { Latitude } \\
\text { (dd }{ }^{\circ} \mathrm{mm} \text { 'ss") }\end{array}$ & $\begin{array}{l}\text { Longitude } \\
\text { (dd }{ }^{\circ} \mathrm{mm} \text { 'ss") }\end{array}$ & $\begin{array}{l}\text { Index } \\
\text { station }^{1}\end{array}$ & $\begin{array}{c}\text { Drainage } \\
\text { area } \\
\left(\mathrm{mi}^{2}\right)\end{array}$ & $\begin{array}{c}\text { Base flow } \\
\text { factor } \\
\left(\mathrm{ft}^{3} / \mathrm{s} / \mathrm{mi}^{2}\right)\end{array}$ & $\begin{array}{l}\text { Base flow } \\
\text { method }\end{array}$ & $\begin{array}{l}\text { Base flow } \\
\qquad\left(\mathrm{ft}^{3} / \mathrm{s}\right)\end{array}$ & $\begin{array}{c}\text { Recharge } \\
\text { (inches) }\end{array}$ \\
\hline 144 & 04085030 & Apple Ck nr Kaukauna & $44^{\circ} 19^{\prime} 17^{\prime \prime}$ & $88^{\circ} 17^{\prime} 00^{\prime \prime}$ & 04079000 & 15.2 & 0.0170 & equation & 1.7 & 1.5 \\
\hline 145 & 04085051 & Apple Ck nr Wrightstown & $44^{\circ} 20^{\prime} 56^{\prime \prime}$ & $88^{\circ} 09^{\prime} 41^{\prime \prime}$ & 04079000 & 48.1 & .0090 & equation & 4.1 & 1.2 \\
\hline 146 & 04085064 & $\begin{array}{l}\text { N Br Ashwaubenon Ck nr } \\
\text { Freedom }\end{array}$ & $44^{\circ} 23^{\prime} 57^{\prime \prime}$ & $88^{\circ} 11^{\prime} 28^{\prime \prime}$ & 04079000 & 3.13 & .0003 & equation & .0 & .2 \\
\hline 147 & 04085070 & Ashwaubenon Ck at De Pere & $44^{\circ} 27^{\prime} 17^{\prime \prime}$ & $88^{\circ} 05^{\prime} 43^{\prime \prime}$ & 04079000 & 26.6 & .0004 & equation & .4 & .2 \\
\hline 148 & 04085076 & Dutchman Ck Tr nr De Pere & $44^{\circ} 28^{\prime} 53^{\prime \prime}$ & $88^{\circ} 07^{\prime} 29^{\prime \prime}$ & 04079000 & 2.41 & .0337 & equation & .4 & 2.1 \\
\hline 149 & 04085080 & Dutchman Ck nr De Pere & $44^{\circ} 28^{\prime} 43^{\prime \prime}$ & $88^{\circ} 04^{\prime} 21^{\prime \prime}$ & 04079000 & 28.1 & .0131 & equation & 2.9 & 1.4 \\
\hline 150 & 04085110 & E R at Co Hwy Pp nr De Pere & $44^{\circ} 24^{\prime} 21^{\prime \prime}$ & $88^{\circ} 03^{\prime} 12^{\prime \prime}$ & 04085200 & 51.6 & .0090 & equation & 4.4 & 1.2 \\
\hline
\end{tabular}

${ }^{1}$ Low-flow discharge measurements at partial-record stations were compared to one or more nearby USGS gaging stations to establish a relation between measured discharge and concurrent daily discharge. The nearby gaging station that provided the best relation with the partial-record station was chosen as the index station and was associated with nearby miscellaneous measurement sites.

${ }^{2}$ Mismatch between surface-water and ground-water drainage area. 


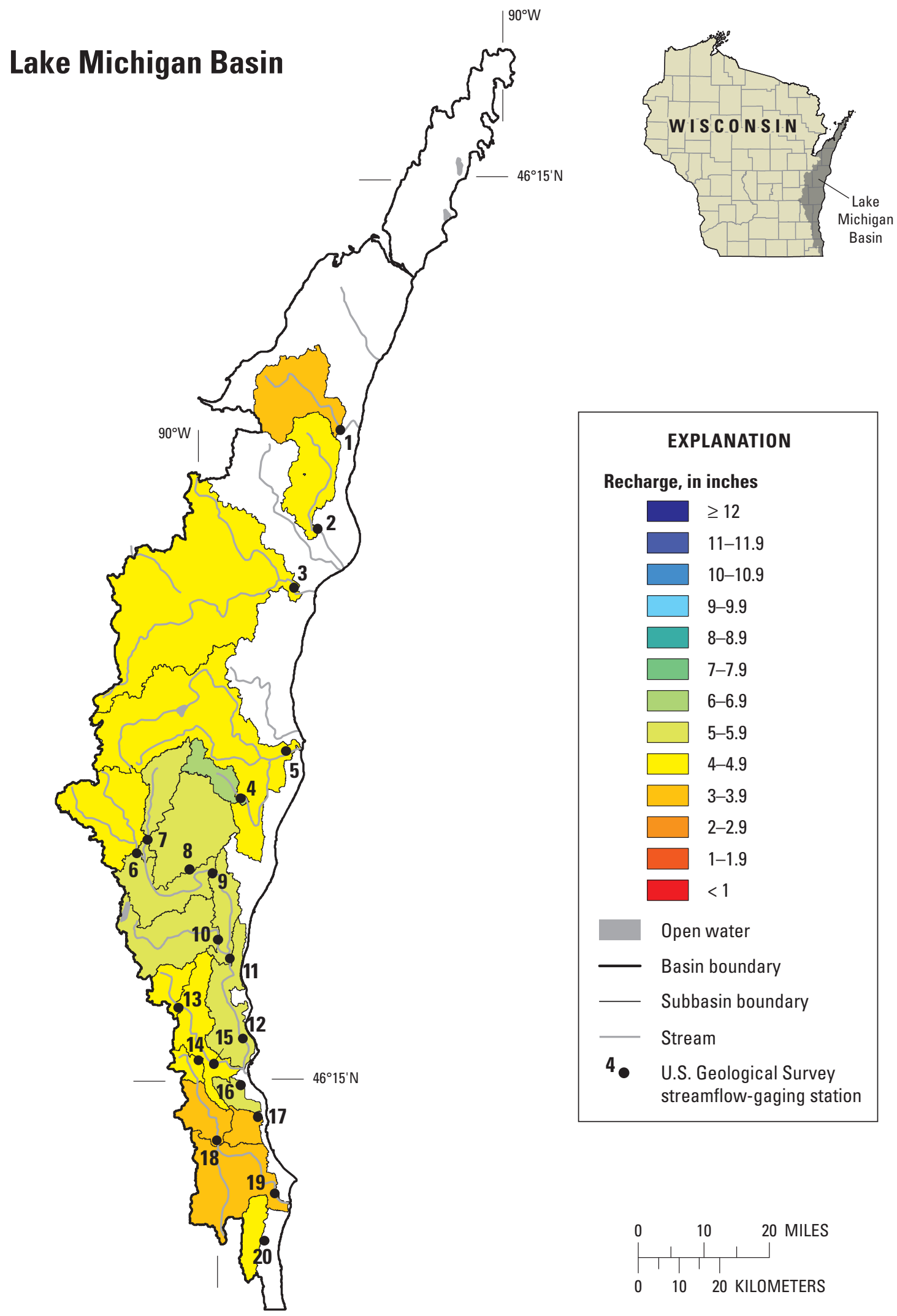

Appendix 9-A. Locations of streamflow-gaging stations in the Lake Michigan Basin and spatial distribution of average annual recharge. 


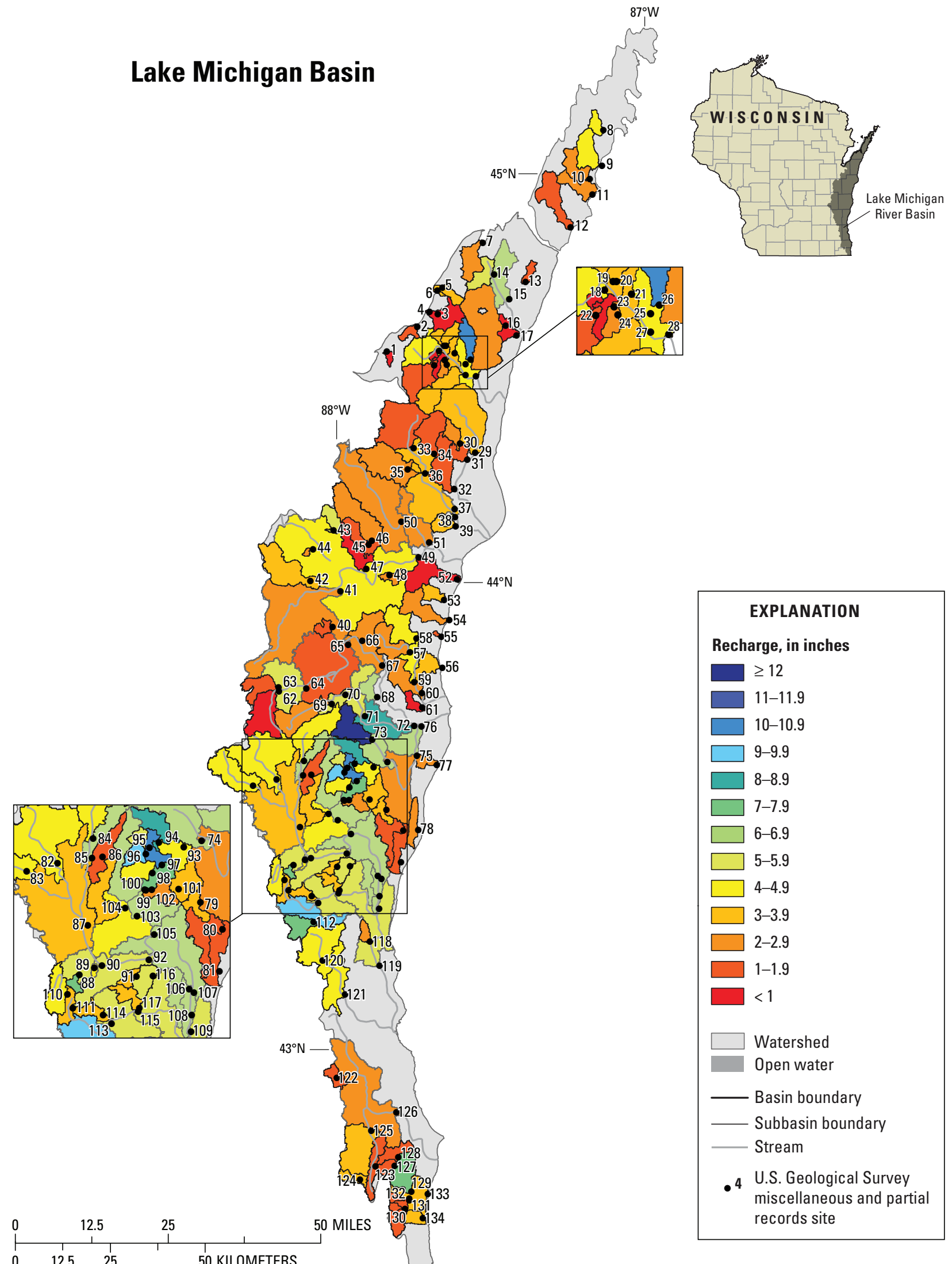

Appendix 9-B. Locations of miscellaneous and partial-record sites in the Lake Michigan Basin and spatial distribution of estimated average annual recharge. 
Appendix 9C. Average annual base flow and corresponding recharge, 1970-99, for streamflow-gaging stations in the Lake Michigan Basin.

[USGS, U.S. Geological Survey; ID, identification number; mi² $^{2}$ square mile; ft³/s, cubic foot per second; BFI, Baseflow Index; ex rec, extended record; R, River; Bk, Brook; Br, Branch; Fk, Fork; Ck, Creek; Tr, Tributary; nr, near; N, North; S, South; E, East; W, West; --, not determined in historical low-flow reports. See references for full list of low-flow reports.]

\begin{tabular}{|c|c|c|c|c|c|c|c|c|c|c|c|}
\hline $\begin{array}{c}\text { Map } \\
\text { number }\end{array}$ & $\begin{array}{l}\text { USGS } \\
\text { station } \\
\text { ID }\end{array}$ & Station name & $\begin{array}{c}\text { Drainage } \\
\text { area } \\
\left(\mathrm{mi}^{2}\right)\end{array}$ & $\begin{array}{c}\text { Period of } \\
\text { analysis } \\
\text { (water years') }\end{array}$ & $\begin{array}{c}\text { Base flow } \\
\text { method }\end{array}$ & $\begin{array}{l}\text { Base flow } \\
\text { index }\end{array}$ & $\begin{array}{l}\text { Base } \\
\text { flow } \\
\left(\mathrm{ft}^{3} / \mathrm{s}\right)\end{array}$ & $\begin{array}{c}\text { Recharge } \\
\text { (inches) }\end{array}$ & $\begin{array}{c}\text { Total } \\
\text { streamflow } \\
\left(\mathrm{ft}^{3} / \mathrm{s}\right)\end{array}$ & $\begin{array}{c}\text { Total } \\
\text { streamflow } \\
\text { (inches) }\end{array}$ & $\begin{array}{l}{ }^{2} 090 \\
\left(\mathrm{ft}^{3} / \mathrm{s}\right)\end{array}$ \\
\hline 1 & 04085200 & Kewaunee R nr Kewaunee & 127 & $1970-1995$ & BFI & 0.4120 & 36 & 3.8 & 91 & 9.7 & 14 \\
\hline 2 & 04085281 & E Twin R at Mishicot & 110 & $1973-1996$ & BFI & .4590 & 35 & 4.3 & -- & -- & 12 \\
\hline 3 & 04085427 & Manitowoc $\mathrm{R}$ at Manitowoc & 526 & 1973-1996 & BFI & .4950 & 160 & 4.3 & 330 & 8.5 & 30 \\
\hline 4 & 04085813 & Onion $\mathrm{R}$ at Hingham & 37.2 & $1979-1980$ & ex rec & -- & 17 & 6.2 & -- & -- & -- \\
\hline 5 & 04086000 & Sheboygan $\mathrm{R}$ at Sheboygan & 418 & 1970-1999 & BFI & .5120 & 150 & 4.7 & 290 & 9.4 & 52 \\
\hline 6 & 04086150 & Milwaukee R at Kewaskum & 138 & 1969-1981 & BFI & -- & 45 & 4.4 & -- & -- & 15 \\
\hline 7 & 04086200 & E Br Milwaukee $\mathrm{R}$ at New Fane & 54.1 & 1969-1981 & BFI & -- & 20 & 5.0 & -- & -- & 8.5 \\
\hline 8 & 04086340 & N Br Milwaukee R nr Fillmore & 148 & 1969-1981 & ex rec & -- & 60 & 5.5 & -- & -- & 28 \\
\hline 9 & 04086360 & Milwaukee R at Waubeka & 432 & $\begin{array}{c}\text { 1969-1981, } \\
1994\end{array}$ & ex rec & -- & 180 & 5.7 & -- & -- & 76 \\
\hline 10 & 04086500 & Cedar Ck nr Cedarburg & 120 & 1970-1999 & BFI & .5240 & 51 & 5.8 & 100 & 11.3 & 19 \\
\hline 11 & 04086600 & Milwaukee R nr Cedarburg & 607 & $1983-1999$ & BFI & .5700 & 260 & 5.8 & 460 & 10.3 & 120 \\
\hline 12 & 04087000 & Milwaukee $\mathrm{R}$ at Milwaukee & 696 & $1970-1999$ & BFI & .5500 & 290 & 5.7 & 530 & 10.4 & 120 \\
\hline 13 & 04087030 & Menomonee R at Menomonee Falls & 34.7 & $1976-1999$ & BFI & .4180 & 12 & 4.7 & 30 & 11.7 & 4.2 \\
\hline 14 & 04087088 & Underwood $\mathrm{Ck}$ at Wauwatosa & 18.2 & 1976-1998 & BFI & .3900 & 6.0 & 4.5 & 15 & 11.2 & 3.1 \\
\hline 15 & 04087120 & Menomonee $\mathrm{R}$ at Wauwatosa & 123 & $1970-1999$ & BFI & .3620 & 40 & 4.4 & 110 & 12.3 & 18 \\
\hline 16 & 04087160 & Kinnickinnic R at Milwaukee & 20.4 & $1977-1982$ & ex rec & -- & 8.0 & 5.3 & -- & -- & -- \\
\hline 17 & 04087204 & Oak Ck at South Milwaukee & 25 & 1970-1999 & BFI & .2560 & 6.0 & 3.3 & 24 & 13.0 & 2.0 \\
\hline 18 & 04087220 & Root R nr Franklin & 49.2 & 1970-1999 & BFI & .3030 & 13 & 3.6 & 45 & 12.4 & 5.0 \\
\hline 19 & 04087240 & Root $\mathrm{R}$ at Racine & 190 & 1970-1999 & BFI & .3220 & 51 & 3.6 & 160 & 11.4 & 11 \\
\hline 20 & 04087257 & Pike R nr Racine & 38.5 & 1972-1999 & BFI & .3590 & 13 & 4.6 & 37 & 13.0 & 5.6 \\
\hline
\end{tabular}

${ }^{1}$ Water year is a 12-month period from October 1 through September 30, and is designated by the calendar year in which it ends (for example, the 2004 water year occurred October 1,2003 through September 30, 2004).

${ }^{2}$ Discharge that is exceeded 90 percent of the time. 
Appendix 9D. Average annual base flow and corresponding recharge, 1970-99, for partial-record stations in the Lake Michigan Basin.

[USGS, U.S. Geological Survey; ID, identification number; $\mathrm{dd}^{\circ} \mathrm{mm}$ 'ss", degrees, minutes, seconds; $\mathrm{mi}^{2}$, square mile; $\mathrm{ft}^{3} / \mathrm{s} / \mathrm{mi}^{2}$, cubic foot per second per square mile; $\mathrm{ft}^{3} / \mathrm{s}$, cubic foot per second; equation, statewide regression equation; relation, established relation line with index station; R, River; Bk, Brook; Br, Branch; Fk, Fork; Ck, Creek; Tr, Tributary; nr, near; N, North; S, South; E, East; W, West; ,-- not determined in historical low-flow reports. See references for full list of low-flow reports.]

\begin{tabular}{|c|c|c|c|c|c|c|c|c|c|c|}
\hline $\begin{array}{c}\text { Map } \\
\text { number }\end{array}$ & $\begin{array}{c}\text { USGS } \\
\text { station ID }\end{array}$ & Station name & $\begin{array}{c}\text { Latitude } \\
\text { (ddºmm'ss") }\end{array}$ & $\begin{array}{l}\text { Longitude } \\
\text { (dd }{ }^{\circ} \mathrm{mm} \text { 'ss") }\end{array}$ & $\begin{array}{l}\text { Index } \\
\text { station }^{1}\end{array}$ & $\begin{array}{c}\text { Drainage } \\
\text { area } \\
\left(\mathrm{mi}^{2}\right)\end{array}$ & $\begin{array}{c}\text { Base flow } \\
\text { factor } \\
\left(\mathrm{ft}^{3} / \mathrm{s} / \mathrm{mi}^{2}\right)\end{array}$ & $\begin{array}{c}\text { Base flow } \\
\text { method }\end{array}$ & $\begin{array}{c}\text { Base flow } \\
\qquad\left(\mathrm{ft}^{3} / \mathrm{s}\right)\end{array}$ & $\begin{array}{c}\text { Recharge } \\
\text { (inches) }\end{array}$ \\
\hline 1 & 040851405 & Lk Mich Tr nr Champion & $44^{\circ} 34^{\prime} 43^{\prime \prime}$ & $87^{\circ} 53^{\prime} 00^{\prime \prime}$ & 04085200 & 12.4 & 0.0032 & equation & 0.6 & 0.6 \\
\hline 2 & 04085143 & Lk Mich Tr nr Dyckesville & $44^{\circ} 38^{\prime} 03^{\prime \prime}$ & $87^{\circ} 47^{\prime} 00^{\prime \prime}$ & 04085200 & 5.19 & .0075 & equation & .4 & ${ }^{2} 1.0$ \\
\hline 3 & 04085146 & Red R nr Dyckesville & $44^{\circ} 39^{\prime} 37^{\prime \prime}$ & $87^{\circ} 43^{\prime} 00^{\prime \prime}$ & 04085200 & 12.8 & .0043 & equation & .7 & ${ }^{2} .8$ \\
\hline 4 & 04085147 & Red R nr Dyckesville & $44^{\circ} 40^{\prime} 04^{\prime \prime}$ & $87^{\circ} 44^{\prime} 36^{\prime \prime}$ & 04085200 & 13.7 & .0053 & equation & .9 & ${ }^{2} .9$ \\
\hline 5 & 04085150 & Renard Ck nr Brussels & $44^{\circ} 43^{\prime} 16^{\prime \prime}$ & $87^{\circ} 42^{\prime} 00^{\prime \prime}$ & 04085200 & 6 & .0792 & equation & 1.5 & 3.4 \\
\hline 6 & 04085154 & Renard Ck nr Brussels & $44^{\circ} 42^{\prime} 54^{\prime \prime}$ & $87^{\circ} 43^{\prime} 00^{\prime \prime}$ & 04085200 & 7.88 & .0661 & equation & 1.8 & 3.1 \\
\hline 7 & 04085158 & Keyes Ck nr Little Sturgeon & $44^{\circ} 49^{\prime} 13^{\prime \prime}$ & $87^{\circ} 34^{\prime} 00^{\prime \prime}$ & 04085200 & 14.4 & .0291 & equation & 2.2 & 2.1 \\
\hline 8 & 04085163 & Heins Ck nr Baileys Harbor & $45^{\circ} 04^{\prime} 08^{\prime \prime}$ & $87^{\circ} 10^{\prime} 00^{\prime \prime}$ & 04085200 & 4.48 & .1663 & equation & 1.6 & 5.0 \\
\hline 9 & 04085170 & Hibbard Ck at Jacksonport & $44^{\circ} 59^{\prime} 16^{\prime \prime}$ & $87^{\circ} 10^{\prime} 31^{\prime \prime}$ & 04085200 & 16.4 & .1510 & equation & 5.4 & 4.5 \\
\hline 10 & 04085171 & Logan Ck nr Jacksonport & $44^{\circ} 57^{\prime} 28^{\prime \prime}$ & $87^{\circ} 13^{\prime} 00^{\prime \prime}$ & 04085200 & 14.7 & .0466 & equation & 2.9 & 22.6 \\
\hline 11 & 04085172 & Whitefish Bay Ck nr Jacksonport & $44^{\circ} 55^{\prime} 22^{\prime \prime}$ & $87^{\circ} 12^{\prime} 00^{\prime \prime}$ & 04085200 & 18.6 & .0285 & equation & 2.8 & ${ }^{2} 2.1$ \\
\hline 12 & 04085174 & Lilly Bay Ck nr Sturgeon Bay & $44^{\circ} 50^{\prime} 59^{\prime \prime}$ & $87^{\circ} 17^{\prime} 00^{\prime \prime}$ & 04085200 & 20.4 & .0084 & equation & 1.6 & ${ }^{2} 1.1$ \\
\hline 13 & 04085179 & Stoney Ck nr Maplewood & $44^{\circ} 43^{\prime} 43^{\prime \prime}$ & $87^{\circ} 26^{\prime} 00^{\prime \prime}$ & 04085200 & 12.6 & .0145 & equation & 1.3 & ${ }^{2} 1.4$ \\
\hline 14 & 04085184 & Ahnapee Ck nr Kolberg & $44^{\circ} 44^{\prime} 55^{\prime \prime}$ & $87^{\circ} 32^{\prime} 00^{\prime \prime}$ & 04085200 & 21.7 & .1751 & equation & 8.5 & 5.3 \\
\hline 15 & 04085186 & Ahnapee $\mathrm{Ck}$ at Forestville & $44^{\circ} 41^{\prime} 24^{\prime \prime}$ & $87^{\circ} 29^{\prime} 14^{\prime \prime}$ & 04085200 & 32 & .1390 & relation & 15.0 & 6.4 \\
\hline 16 & 04085190 & Silver Ck nr Algoma & $44^{\circ} 37^{\prime} 49^{\prime \prime}$ & $87^{\circ} 30^{\prime} 03^{\prime \prime}$ & 04085200 & 58.2 & -- & relation & 10.0 & 22.3 \\
\hline 17 & 040851906 & Silver Ck nr Algoma & $44^{\circ} 366^{\prime} 27^{\prime \prime}$ & $87^{\circ} 28^{\prime} 00^{\prime \prime}$ & 04085200 & 63.1 & .0012 & equation & 1.9 & ${ }^{2} .4$ \\
\hline 18 & 04085193 & Kewaunee R Tr nr Luxemburg & $44^{\circ} 34^{\prime} 37^{\prime \prime}$ & $87^{\circ} 43^{\prime} 00^{\prime \prime}$ & 04085200 & 10.6 & .0034 & equation & .5 & 2.7 \\
\hline 19 & 040851933 & Kewaunne R nr Luxemburg & $44^{\circ} 35^{\prime} 19^{\prime \prime}$ & $87^{\circ} 42^{\prime} 00^{\prime \prime}$ & 04085200 & 20.8 & .0371 & equation & 3.6 & 2.4 \\
\hline 20 & 040851939 & Kewaunee R Tr nr Luxemburg & $44^{\circ} 35^{\prime} 19^{\prime \prime}$ & $87^{\circ} 41^{\prime} 00^{\prime \prime}$ & 04085200 & 5.96 & .0847 & equation & 1.6 & 3.5 \\
\hline 21 & 040851943 & Kewaunee R nr Luxemburg & $44^{\circ} 34^{\prime} 18^{\prime \prime}$ & $87^{\circ} 40^{\prime} 00^{\prime \prime}$ & 04085200 & 31.5 & .0605 & equation & 7.1 & 3.1 \\
\hline 22 & 040851947 & School Ck nr Luxemburg & $44^{\circ} 32^{\prime} 42^{\prime \prime}$ & $87^{\circ} 44^{\prime} 00^{\prime \prime}$ & 04085200 & 14.6 & .0144 & equation & 1.5 & ${ }^{2} 1.4$ \\
\hline 23 & 040851951 & School Ck nr Luxemburg & $44^{\circ} 33^{\prime} 20^{\prime \prime}$ & $87^{\circ} 42^{\prime} 00^{\prime \prime}$ & 04085200 & 23.7 & .0054 & equation & 1.5 & 2.9 \\
\hline 24 & 040851955 & Luxemburg Ck at Luxemburg & $44^{\circ} 32^{\prime} 41^{\prime \prime}$ & $87^{\circ} 41^{\prime} 00^{\prime \prime}$ & 04085200 & 2.78 & .0780 & relation & .6 & 2.9 \\
\hline
\end{tabular}

${ }^{1}$ Low-flow discharge measurements at partial-record stations were compared to one or more nearby USGS gaging stations to establish a relation between measured discharge and concurrent daily discharge. The nearby gaging station that provided the best relation with the partial-record station was chosen as the index station and was associated with nearby miscellaneous measurement sites.

${ }^{2}$ Mismatch between surface-water and ground-water drainage area. 
Appendix 9D. Average annual base flow and corresponding recharge, 1970-99, for partial-record stations in the Lake Michigan Basin-Continued.

[USGS, U.S. Geological Survey; ID, identification number; $\mathrm{dd}^{\circ} \mathrm{mm}$ 'ss", degrees, minutes, seconds; $\mathrm{mi}^{2}$, square mile; $\mathrm{ft}^{3} / \mathrm{s} / \mathrm{mi}^{2}$, cubic foot per second per square mile; $\mathrm{ft}^{3} / \mathrm{s}$, cubic foot per second; equation, statewide regression equation; relation, established relation line with index station; R, River; Bk, Brook; Br, Branch; Fk, Fork; Ck, Creek; Tr, Tributary; nr, near; N, North; S, South; E, East; W, West; ,-- not determined in historical low-flow reports. See references for full list of low-flow reports.]

\begin{tabular}{|c|c|c|c|c|c|c|c|c|c|c|}
\hline $\begin{array}{c}\text { Map } \\
\text { number }\end{array}$ & $\begin{array}{c}\text { USGS } \\
\text { station ID }\end{array}$ & Station name & $\begin{array}{c}\text { Latitude } \\
\text { (dd }{ }^{\circ} \mathrm{mm} \text { 'ss") }\end{array}$ & $\begin{array}{l}\text { Longitude } \\
\text { (ddºmm'ss") }\end{array}$ & $\begin{array}{l}\text { Index } \\
\text { station }^{1}\end{array}$ & $\begin{array}{c}\text { Drainage } \\
\text { area } \\
\left(\mathrm{mi}^{2}\right)\end{array}$ & $\begin{array}{c}\text { Base flow } \\
\text { factor } \\
\left(\mathrm{ft}^{3} / \mathrm{s} / \mathrm{mi}^{2}\right)\end{array}$ & $\begin{array}{l}\text { Base flow } \\
\text { method }\end{array}$ & $\begin{array}{c}\text { Base flow } \\
\left(\mathrm{ft}^{3} / \mathrm{s}\right)\end{array}$ & $\begin{array}{c}\text { Recharge } \\
\text { (inches) }\end{array}$ \\
\hline 25 & 040851959 & Kewaunee R nr Luxemburg & $44^{\circ} 32^{\prime} 41^{\prime \prime}$ & $87^{\circ} 38^{\prime} 00^{\prime \prime}$ & 04085200 & 67.2 & 0.0582 & equation & 15.1 & 3.1 \\
\hline 26 & 040851967 & Casco $\mathrm{Ck}$ at Casco & $44^{\circ} 33^{\prime} 21^{\prime \prime}$ & $87^{\circ} 37^{\prime} 00^{\prime \prime}$ & 04085200 & 10.1 & .0830 & relation & 8.0 & 10.8 \\
\hline 27 & 040851983 & Scarboro Ck nr Luxemburg & $44^{\circ} 31^{\prime} 16^{\prime \prime}$ & $87^{\circ} 38^{\prime} 00^{\prime \prime}$ & 04085200 & 20 & .0910 & equation & 5.5 & 3.8 \\
\hline 28 & 040851990 & Kewaunee R nr Luxemburg & $44^{\circ} 31^{\prime} 00^{\prime \prime}$ & $87^{\circ} 36^{\prime} 00^{\prime \prime}$ & 04085200 & 112 & .1049 & equation & 34.6 & 4.2 \\
\hline 29 & 04085244 & E Twin R nr Tisch Mills & $44^{\circ} 20^{\prime} 32^{\prime \prime}$ & $87^{\circ} 36^{\prime} 37^{\prime \prime}$ & 04085200 & 49.7 & .1070 & relation & 14.0 & 3.8 \\
\hline 30 & 04085250 & E Twin R Tr nr Stangelville & $44^{\circ} 21^{\prime} 51^{\prime \prime}$ & $87^{\circ} 39^{\prime} 29^{\prime \prime}$ & 04085200 & 7.43 & .0316 & equation & 1.2 & 2.1 \\
\hline 31 & 040852508 & Tisch Mills Ck at Tisch Mills & $44^{\circ} 19^{\prime} 40^{\prime \prime}$ & $87^{\circ} 38^{\prime} 10^{\prime \prime}$ & 04085200 & 16.3 & .0239 & equation & 2.2 & 1.9 \\
\hline 32 & 04085270 & Jambo Ck nr Mishicot & $44^{\circ} 15^{\prime} 43^{\prime \prime}$ & $87^{\circ} 40^{\prime} 52^{\prime \prime}$ & 04085200 & 19.3 & .0204 & equation & 2.5 & 1.7 \\
\hline 33 & 04085306 & Neshoto R nr Denmark & $44^{\circ} 21^{\prime} 26^{\prime \prime}$ & $87^{\circ} 48^{\prime} 22^{\prime \prime}$ & 04085200 & 39.2 & .1020 & relation & 3.2 & 1.1 \\
\hline 34 & 04085314 & Buck Ck nr Cooperstown & $44^{\circ} 20^{\prime} 30^{\prime \prime}$ & $87^{\circ} 44^{\prime} 30^{\prime \prime}$ & 04085200 & 20.1 & .0146 & equation & 2.1 & 1.5 \\
\hline 35 & 04085322 & Devils R nr Denmark & $44^{\circ} 18^{\prime} 31^{\prime \prime}$ & $87^{\circ} 49^{\prime} 36^{\prime \prime}$ & 04085200 & 29.6 & .0338 & equation & 4.9 & 2.3 \\
\hline 36 & 04085323 & Devils R nr Denmark & $44^{\circ} 17^{\prime} 52^{\prime \prime}$ & $87^{\circ} 46^{\prime} 19^{\prime \prime}$ & 04085200 & 36.3 & .0790 & equation & 9.4 & 3.5 \\
\hline 37 & 04085330 & W Twin R nr Francis Ck & $44^{\circ} 12^{\prime} 54^{\prime \prime}$ & $87^{\circ} 40^{\prime} 50^{\prime \prime}$ & 04085200 & 150 & .0940 & relation & 39.8 & 3.6 \\
\hline 38 & 040853315 & W Twin R Tr nr Francis Ck & $44^{\circ} 11^{\prime} 48^{\prime \prime}$ & $87^{\circ} 40^{\prime} 50^{\prime \prime}$ & 04085200 & 2.43 & .1785 & equation & .9 & 5.1 \\
\hline 39 & 04085334 & W Twin R Tr nr Rockwood & $44^{\circ} 10^{\prime} 33^{\prime \prime}$ & $87^{\circ} 40^{\prime} 47^{\prime \prime}$ & 04085200 & 4.88 & -- & relation & 1.2 & 3.3 \\
\hline 40 & 04085392 & Jordan Ck at New Holstein & $43^{\circ} 57^{\prime} 12^{\prime \prime}$ & $88^{\circ} 04^{\prime} 35^{\prime \prime}$ & 04086000 & 3.3 & .0154 & equation & .4 & 1.4 \\
\hline 41 & 04085396 & S Br Manitowoc R nr Collins & $44^{\circ} 02^{\prime} 01^{\prime \prime}$ & $88^{\circ} 03^{\prime} 00^{\prime \prime}$ & 04086000 & 114 & .0511 & equation & 24.2 & 2.9 \\
\hline 42 & 04085400 & Killsnake R nr Chilton & $44^{\circ} 03^{\prime} 33^{\prime \prime}$ & $88^{\circ} 08^{\prime} 36^{\prime \prime}$ & 04086000 & 29.4 & .0480 & relation & 8.5 & 3.9 \\
\hline 43 & 04085403 & Spring $\mathrm{Ck}$ at Brillion & $44^{\circ} 10^{\prime} 22^{\prime \prime}$ & $88^{\circ} 04^{\prime} 00^{\prime \prime}$ & 04086000 & 8.17 & .1630 & equation & 3.0 & 5.0 \\
\hline 44 & 04085404 & N Br Manitowoc R Tr at Hilbert & $44^{\circ} 07^{\prime} 53^{\prime \prime}$ & $88^{\circ} 08^{\prime} 00^{\prime \prime}$ & 04086000 & 5.3 & .0494 & equation & 1.0 & 2.7 \\
\hline 45 & 04085408 & Mud Ck at Reeddsville & $44^{\circ} 08^{\prime} 54^{\prime \prime}$ & $87^{\circ} 56^{\prime} 46^{\prime \prime}$ & 04086000 & 32.2 & .0283 & equation & 4.9 & 2.1 \\
\hline 46 & 04085410 & Mud Ck nr Reedsville & $44^{\circ} 08^{\prime} 21^{\prime \prime}$ & $87^{\circ} 57^{\prime} 26^{\prime \prime}$ & 04086000 & 42 & .0110 & relation & 5.5 & 1.8 \\
\hline 47 & 040854103 & Mud Ck nr Collins & $44^{\circ} 05^{\prime} 03^{\prime \prime}$ & $87^{\circ} 57^{\prime} 58^{\prime \prime}$ & 04086000 & 58.8 & .0005 & equation & 1.1 & ${ }^{2} .3$ \\
\hline 48 & 040854108 & Manitowoc R Tr at Valders & $44^{\circ} 04^{\prime} 05^{\prime \prime}$ & $87^{\circ} 53^{\prime} 33^{\prime \prime}$ & 04086000 & 5.06 & .0571 & equation & 1.1 & 2.9 \\
\hline 49 & 04085415 & Manitowoc R nr Clark Mills & $44^{\circ} 06^{\prime} 27^{\prime \prime}$ & $87^{\circ} 47^{\prime} 57^{\prime \prime}$ & 04086000 & 410 & .0648 & equation & 101.1 & 3.3 \\
\hline
\end{tabular}

${ }^{1}$ Low-flow discharge measurements at partial-record stations were compared to one or more nearby USGS gaging stations to establish a relation between measured discharge and concurrent daily discharge. The nearby gaging station that provided the best relation with the partial-record station was chosen as the index station and was associated with nearby miscellaneous measurement sites.

${ }^{2}$ Mismatch between surface-water and ground-water drainage area. 
Appendix 9D. Average annual base flow and corresponding recharge, 1970-99, for partial-record stations in the Lake Michigan Basin-Continued.

[USGS, U.S. Geological Survey; ID, identification number; $\mathrm{dd}^{\circ} \mathrm{mm}$ 'ss", degrees, minutes, seconds; $\mathrm{mi}^{2}$, square mile; $\mathrm{ft}^{3} / \mathrm{s} / \mathrm{mi}^{2}$, cubic foot per second per square mile; $\mathrm{ft}^{3} / \mathrm{s}, \mathrm{cubic} \mathrm{foot}$ per second; equation, statewide regression equation; relation, established relation line with index station; R, River; Bk, Brook; Br, Branch; Fk, Fork; Ck, Creek; Tr, Tributary; nr, near; N, North; S, South; E, East; W, West;

,-- not determined in historical low-flow reports. See references for full list of low-flow reports.]

\begin{tabular}{|c|c|c|c|c|c|c|c|c|c|c|}
\hline $\begin{array}{c}\text { Map } \\
\text { number }\end{array}$ & $\begin{array}{c}\text { USGS } \\
\text { station ID }\end{array}$ & Station name & $\begin{array}{c}\text { Latitude } \\
\text { (dd }{ }^{\circ} \mathrm{mm} \text { 'ss") }\end{array}$ & $\begin{array}{c}\text { Longitude } \\
\text { (dd }{ }^{\circ} \mathrm{mm} \text { 'ss") }\end{array}$ & $\begin{array}{l}\text { Index } \\
\text { station }{ }^{1}\end{array}$ & $\begin{array}{c}\text { Drainage } \\
\text { area } \\
\left(\mathrm{mi}^{2}\right) \\
\end{array}$ & $\begin{array}{c}\text { Base flow } \\
\text { factor } \\
\left(\mathrm{ft}^{3} / \mathrm{s} / \mathrm{mi}^{2}\right)\end{array}$ & $\begin{array}{c}\text { Base flow } \\
\text { method }\end{array}$ & $\begin{array}{c}\text { Base flow } \\
\left(\mathrm{ft}^{3} / \mathrm{s}\right)\end{array}$ & $\begin{array}{c}\text { Recharge } \\
\text { (inches) }\end{array}$ \\
\hline 50 & 04085420 & Branch R nr Cato & $44^{\circ} 11^{\prime} 20^{\prime \prime}$ & $87^{\circ} 51^{\prime} 04^{\prime \prime}$ & 04085200 & 78.8 & 0.0720 & relation & 15.1 & 2.6 \\
\hline 51 & 040854245 & Branch $\mathrm{R}$ at $\mathrm{Br}$ & $44^{\circ} 08^{\prime} 26^{\prime \prime}$ & $87^{\circ} 45^{\prime} 55^{\prime \prime}$ & 04085200 & 106 & .0416 & equation & 20.2 & 2.6 \\
\hline 52 & 04085435 & Silver Ck nr Manitowoc & $44^{\circ} 03^{\prime} 17^{\prime \prime}$ & $87^{\circ} 40^{\prime} 50^{\prime \prime}$ & 04086000 & 21.2 & .0061 & equation & 1.4 & 2.9 \\
\hline 53 & 04085438 & Pine $\mathrm{Ck}$ at Newton & $44^{\circ} 00^{\prime} 31^{\prime \prime}$ & $87^{\circ} 43^{\prime} 19^{\prime \prime}$ & 04086000 & 6.6 & -- & relation & 1.7 & 3.4 \\
\hline 54 & 04085440 & Point Ck nr Newton & $43^{\circ} 57^{\prime} 43^{\prime \prime}$ & $87^{\circ} 42^{\prime} 31^{\prime \prime}$ & 04086000 & 18.7 & .0474 & equation & 3.7 & 2.7 \\
\hline 55 & 040854423 & Centerville $\mathrm{Ck}$ at Centerville & $43^{\circ} 55^{\prime} 30^{\prime \prime}$ & $87^{\circ} 44^{\prime} 00^{\prime \prime}$ & 04086000 & 3.12 & .0217 & equation & .4 & ${ }^{2} 1.7$ \\
\hline 56 & 04085445 & Sevenmile $\mathrm{Ck}$ at Haven & $43^{\circ} 51^{\prime} 14^{\prime \prime}$ & $87^{\circ} 44^{\prime} 00^{\prime \prime}$ & 04086000 & 5.6 & .0622 & equation & 1.2 & 3.0 \\
\hline 57 & 04085450 & Pigeon R nr Howards Grove & $43^{\circ} 53^{\prime} 30^{\prime \prime}$ & $87^{\circ} 50^{\prime} 02^{\prime \prime}$ & 04086000 & 18.5 & .0426 & equation & 3.4 & 2.5 \\
\hline 58 & 04085454 & Meeme Ck nr Cleveland & $43^{\circ} 55^{\prime} 20^{\prime \prime}$ & $87^{\circ} 48^{\prime} 45^{\prime \prime}$ & 04086000 & 18.2 & .1101 & equation & 5.6 & 4.1 \\
\hline 59 & 04085458 & Pigeon R at Howards Grove & $43^{\circ} 49^{\prime} 20^{\prime \prime}$ & $87^{\circ} 49^{\prime} 28^{\prime \prime}$ & 04086000 & 48.7 & .1110 & equation & 15.2 & 4.2 \\
\hline 60 & 04085460 & Pigeon R nr Howards Grove & $43^{\circ} 47^{\prime} 49^{\prime \prime}$ & $87^{\circ} 48^{\prime} 02^{\prime \prime}$ & 04086000 & 65 & .0370 & equation & 11.6 & 2.4 \\
\hline 61 & 04085462 & Pigeon R Tr nr Kohler & $43^{\circ} 45^{\prime} 50^{\prime \prime}$ & $87^{\circ} 48^{\prime} 00^{\prime \prime}$ & 04086000 & 4.57 & .0035 & equation & .2 & .7 \\
\hline 62 & 04085480 & Sheboygan R nr Fond du Lac & $43^{\circ} 48^{\prime} 33^{\prime \prime}$ & $88^{\circ} 15^{\prime} 04^{\prime \prime}$ & 04086000 & 27.4 & .0020 & equation & .9 & .5 \\
\hline 63 & 040854801 & Sheboygan $\mathrm{R}$ Tr at Mtcalvary & $43^{\circ} 49^{\prime} 06^{\prime \prime}$ & $88^{\circ} 15^{\prime} 05^{\prime \prime}$ & 04086000 & 12.7 & .0109 & equation & 1.2 & 1.2 \\
\hline 64 & 04085482 & Sheboygan R at St Cloud & $43^{\circ} 48^{\prime} 51^{\prime \prime}$ & $88^{\circ} 09^{\prime} 52^{\prime \prime}$ & 04086000 & 73 & .0620 & relation & 16.2 & 3.0 \\
\hline 65 & 04085497 & Sheboygan $\mathrm{R}$ at Kiel & $43^{\circ} 54^{\prime} 43^{\prime \prime}$ & $88^{\circ} 01^{\prime} 45^{\prime \prime}$ & 04086000 & 153 & .0150 & relation & 12.6 & 1.1 \\
\hline 66 & 04085510 & Sheboygan R Tr nr Kiel & $43^{\circ} 55^{\prime} 12^{\prime \prime}$ & $87^{\circ} 59^{\prime} 02^{\prime \prime}$ & 04086000 & 15.4 & .0431 & equation & 2.9 & 2.5 \\
\hline 67 & 04085600 & Sheboygan R nr Lk Mich & $43^{\circ} 51^{\prime} 46^{\prime \prime}$ & $87^{\circ} 55^{\prime} 22^{\prime \prime}$ & 04086000 & 185 & .0524 & equation & 40.2 & 3.0 \\
\hline 68 & 04085700 & Sheboygan R Tr nr Plymouth & $43^{\circ} 47^{\prime} 26^{\prime \prime}$ & $87^{\circ} 56^{\prime} 31^{\prime \prime}$ & 04086000 & 6.51 & .3150 & relation & 2.9 & 6.1 \\
\hline 69 & 04085748 & Mullet R at Greenbush & $43^{\circ} 46^{\prime} 39^{\prime \prime}$ & $88^{\circ} 05^{\prime} 05^{\prime \prime}$ & 04086000 & 24.4 & .0471 & equation & 4.8 & 2.7 \\
\hline 70 & 04085751 & Mullet $\mathrm{R}$ at Glenbeulah & $43^{\circ} 47^{\prime} 51^{\prime \prime}$ & $88^{\circ} 02^{\prime} 28^{\prime \prime}$ & 04086000 & 30 & -- & relation & 13.2 & 6.0 \\
\hline 71 & 04085770 & Mullet R at Plymouth & $43^{\circ} 44^{\prime} 50^{\prime \prime}$ & $87^{\circ} 58^{\prime} 52^{\prime \prime}$ & 04086000 & 53 & .1919 & equation & 22.1 & 5.7 \\
\hline 72 & 04085778 & Mullet R at Sheboygan Falls & $43^{\circ} 43^{\prime} 21^{\prime \prime}$ & $87^{\circ} 49^{\prime} 37^{\prime \prime}$ & 04086000 & 78.2 & .2047 & relation & 50.0 & 8.7 \\
\hline 73 & 04085800 & Onion R nr Waldo & $43^{\circ} 41^{\prime} 39^{\prime \prime}$ & $87^{\circ} 57^{\prime} 37^{\prime \prime}$ & 04086000 & 20.7 & .6140 & relation & 20.5 & 13.5 \\
\hline 74 & 04085810 & Onion $\mathrm{R}$ at Hingham & $43^{\circ} 38^{\prime} 28^{\prime \prime}$ & $87^{\circ} 54^{\prime} 53^{\prime \prime}$ & 04086000 & 36.2 & -- & relation & 16.0 & 6.0 \\
\hline
\end{tabular}

${ }^{1}$ Low-flow discharge measurements at partial-record stations were compared to one or more nearby USGS gaging stations to establish a relation between measured discharge and concurrent daily discharge. The nearby gaging station that provided the best relation with the partial-record station was chosen as the index station and was associated with nearby miscellaneous measurement sites.

${ }^{2}$ Mismatch between surface-water and ground-water drainage area. 
[USGS, U.S. Geological Survey; ID, identification number; $\mathrm{dd}^{\circ} \mathrm{mm}$ 'ss", degrees, minutes, seconds; $\mathrm{mi}^{2}$, square mile; $\mathrm{ft}^{3} / \mathrm{s} / \mathrm{mi}^{2}$, cubic foot per second per square mile; $\mathrm{ft}^{3} / \mathrm{s}$, cubic foot per second; equation, statewide regression equation; relation, established relation line with index station; R, River; Bk, Brook; Br, Branch; Fk, Fork; Ck, Creek; Tr, Tributary; nr, near; N, North; S, South; E, East; W, West;

--, not determined in historical low-flow reports. See references for full list of low-flow reports.]

\begin{tabular}{|c|c|c|c|c|c|c|c|c|c|c|}
\hline $\begin{array}{c}\text { Map } \\
\text { number }\end{array}$ & $\begin{array}{l}\text { USGS } \\
\text { station ID }\end{array}$ & Station name & $\begin{array}{c}\text { Latitude } \\
\text { (dd }{ }^{\circ} \mathrm{mm} \text { 'ss") }\end{array}$ & $\begin{array}{l}\text { Longitude } \\
\text { (dd }{ }^{\circ} \mathrm{mm} \text { 'ss") }\end{array}$ & $\begin{array}{l}\text { Index } \\
\text { station }{ }^{1}\end{array}$ & $\begin{array}{c}\text { Drainage } \\
\text { area } \\
\left(\mathrm{mi}^{2}\right)\end{array}$ & $\begin{array}{c}\text { Base flow } \\
\text { factor } \\
\left(\mathrm{ft}^{3} / \mathrm{s} / \mathrm{mi}^{2}\right)\end{array}$ & $\begin{array}{l}\text { Base flow } \\
\text { method }\end{array}$ & $\begin{array}{c}\text { Base flow } \\
\quad\left(\mathrm{ft}^{3} / \mathrm{s}\right)\end{array}$ & $\begin{array}{c}\text { Recharge } \\
\text { (inches) }\end{array}$ \\
\hline 75 & 04085830 & Onion $\mathrm{R}$ at Gibbsville & $43^{\circ} 39^{\prime} 16^{\prime \prime}$ & $87^{\circ} 49^{\prime} 18^{\prime \prime}$ & 04086000 & 82 & 0.2050 & relation & 24.0 & 4.0 \\
\hline 76 & 04085950 & Onion $\mathrm{R}$ at Sheboygan Falls & $43^{\circ} 43^{\prime} 16^{\prime \prime}$ & $87^{\circ} 48^{\prime} 19^{\prime \prime}$ & 04086000 & 98 & .1184 & equation & 32.1 & 4.5 \\
\hline 77 & 04086005 & Black R nr Oostburg & $43^{\circ} 37^{\prime} 56^{\prime \prime}$ & $87^{\circ} 45^{\prime} 32^{\prime \prime}$ & 04086000 & 5.38 & .0558 & equation & 1.1 & 2.8 \\
\hline 78 & 04086013 & Sucker Ck nr Lk Church & $43^{\circ} 29^{\prime} 05^{\prime \prime}$ & $87^{\circ} 49^{\prime} 22^{\prime \prime}$ & 04087000 & 6.35 & .0486 & equation & 1.2 & 2.7 \\
\hline 79 & 040860146 & Sauk Ck Tr nr Random Lk & $43^{\circ} 31^{\prime} 59^{\prime \prime}$ & $87^{\circ} 55^{\prime} 16^{\prime \prime}$ & 04087000 & 2.68 & .0770 & equation & .7 & 3.3 \\
\hline 80 & 04086015 & Sauk Ck Tr nr Random Lk & $43^{\circ} 29^{\prime} 05^{\prime \prime}$ & $87^{\circ} 52^{\prime} 13^{\prime \prime}$ & 04087000 & 17.9 & .0260 & equation & 2.6 & 2.0 \\
\hline 81 & 04086020 & Sauk Ck at Port Washington & $43^{\circ} 24^{\prime} 45^{\prime \prime}$ & $87^{\circ} 52^{\prime} 49^{\prime \prime}$ & 04087000 & 27.4 & .0160 & equation & 2.5 & ${ }^{2} 1.2$ \\
\hline 82 & 04086100 & Milwaukee R at Campbellsport & $43^{\circ} 36^{\prime} 26^{\prime \prime}$ & $88^{\circ} 15^{\prime} 52^{\prime \prime}$ & 05424000 & 51.4 & .0120 & relation & 17.0 & 4.5 \\
\hline 83 & 04086130 & $\begin{array}{l}\text { W Br Milwaukee R nr Campbell- } \\
\text { sport }\end{array}$ & $43^{\circ} 35^{\prime} 40^{\prime \prime}$ & $88^{\circ} 20^{\prime} 20^{\prime \prime}$ & 04087000 & 27.4 & .1159 & equation & 8.7 & 4.3 \\
\hline 84 & 04086170 & E Br Milwaukee R at Dundee & $43^{\circ} 38^{\prime} 58^{\prime \prime}$ & $88^{\circ} 10^{\prime} 36^{\prime \prime}$ & 04086200 & 26.3 & .1541 & equation & 9.6 & 5.0 \\
\hline 85 & 04086177 & E Br Milwaukee R nr Dundee & $43^{\circ} 36^{\prime} 58^{\prime \prime}$ & $88^{\circ} 10^{\prime} 52^{\prime \prime}$ & 04087000 & 36.2 & -- & relation & 16.0 & 6.0 \\
\hline 86 & 04086185 & E Br Milwaukee R nr Beechwood & $43^{\circ} 36^{\prime} 58^{\prime \prime}$ & $88^{\circ} 09^{\prime} 16^{\prime \prime}$ & 04086200 & 8.45 & .0197 & equation & 1.0 & 1.7 \\
\hline 87 & 04086210 & Milwaukee R at Kewaskum & $43^{\circ} 29^{\prime} 51^{\prime \prime}$ & $88^{\circ} 11^{\prime} 41^{\prime \prime}$ & 04087000 & 215 & .0907 & equation & 62.3 & 3.9 \\
\hline 88 & 04086226 & Silver Ck nr W Bend & $43^{\circ} 24^{\prime} 41^{\prime \prime}$ & $88^{\circ} 13^{\prime} 00^{\prime \prime}$ & 04087000 & 2.34 & .3899 & equation & 1.3 & 7.7 \\
\hline 89 & 04086237 & Milwaukee R at W Bend & $43^{\circ} 25^{\prime} 21^{\prime \prime}$ & $88^{\circ} 10^{\prime} 52^{\prime \prime}$ & 04087000 & 236 & -- & relation & 94.0 & 5.4 \\
\hline 90 & 04086240 & Milwaukee R nr W Bend & $43^{\circ} 25^{\prime} 34^{\prime \prime}$ & $88^{\circ} 09^{\prime} 42^{\prime \prime}$ & 04087000 & 238 & .1368 & equation & 85.6 & 4.9 \\
\hline 91 & 04086260 & Milwaukee R Tr nr Newburg & $43^{\circ} 24^{\prime} 23^{\prime \prime}$ & $88^{\circ} 04^{\prime} 49^{\prime \prime}$ & 04087000 & 2.03 & .0840 & equation & .5 & 3.4 \\
\hline 92 & 04086270 & Milwaukee $\mathrm{R}$ at Newburg & $43^{\circ} 26^{\prime} 04^{\prime \prime}$ & $88^{\circ} 02^{\prime} 55^{\prime \prime}$ & 04087000 & 265 & -- & relation & 110.0 & 5.6 \\
\hline 93 & 04086280 & N Br Milwaukee R Tr at Adell & $43^{\circ} 37^{\prime} 50^{\prime \prime}$ & $87^{\circ} 57^{\prime} 29^{\prime \prime}$ & 04087000 & 4.23 & .1210 & relation & 1.4 & 4.5 \\
\hline 94 & 04086285 & N Br Milwaukee R nr Cascade & $43^{\circ} 38^{\prime} 25^{\prime \prime}$ & $88^{\circ} 01^{\prime} 03^{\prime \prime}$ & 04087000 & 19.5 & .3880 & equation & 11.5 & 8.0 \\
\hline 95 & 04086292 & Chambers Ck nr Cascade & $43^{\circ} 37^{\prime} 51^{\prime \prime}$ & $88^{\circ} 02^{\prime} 29^{\prime \prime}$ & 04087000 & 2.89 & .1920 & equation & 1.1 & 5.3 \\
\hline 96 & 04086296 & Melius Ck nr Batavia & $43^{\circ} 37^{\prime} 12^{\prime \prime}$ & $88^{\circ} 03^{\prime} 00^{\prime \prime}$ & 04087000 & 4.02 & .6329 & equation & 3.0 & 10.0 \\
\hline 97 & 04086300 & N Br Milwaukee R nr Cascade & $43^{\circ} 36^{\prime} 03^{\prime \prime}$ & $88^{\circ} 00^{\prime} 43^{\prime \prime}$ & 04087000 & 34.2 & .4650 & equation & 26.0 & 10.3 \\
\hline 98 & 04086306 & Batavia Ck nr Batavia & $43^{\circ} 35^{\prime} 11^{\prime \prime}$ & $88^{\circ} 02^{\prime} 07^{\prime \prime}$ & 04087000 & 6.94 & .1267 & equation & 2.2 & 4.4 \\
\hline
\end{tabular}

${ }^{1}$ Low-flow discharge measurements at partial-record stations were compared to one or more nearby USGS gaging stations to establish a relation between measured discharge and concurrent daily discharge. The nearby gaging station that provided the best relation with the partial-record station was chosen as the index station and was associated with nearby miscellaneous measurement sites.

${ }^{2}$ Mismatch between surface-water and ground-water drainage area. 
Appendix 9D. Average annual base flow and corresponding recharge, 1970-99, for partial-record stations in the Lake Michigan Basin-Continued.

[USGS, U.S. Geological Survey; ID, identification number; $\mathrm{dd}^{\circ} \mathrm{mm}$ 'ss", degrees, minutes, seconds; $\mathrm{mi}^{2}$, square mile; $\mathrm{ft}^{3} / \mathrm{s} / \mathrm{mi}^{2}$, cubic foot per second per square mile; $\mathrm{ft}^{3} / \mathrm{s}$, cubic foot per second; equation, statewide regression equation; relation, established relation line with index station; R, River; Bk, Brook; Br, Branch; Fk, Fork; Ck, Creek; Tr, Tributary; nr, near; N, North; S, South; E, East; W, West; ,-- not determined in historical low-flow reports. See references for full list of low-flow reports.]

\begin{tabular}{|c|c|c|c|c|c|c|c|c|c|c|}
\hline $\begin{array}{c}\text { Map } \\
\text { number }\end{array}$ & $\begin{array}{l}\text { USGS } \\
\text { station ID }\end{array}$ & Station name & $\begin{array}{c}\text { Latitude } \\
\text { (dd }{ }^{\circ} \mathrm{mm} \text { 'ss") }\end{array}$ & $\begin{array}{l}\text { Longitude } \\
\text { (dd }{ }^{\circ} \mathrm{mm} \text { 'ss") }\end{array}$ & $\begin{array}{l}\text { Index } \\
\text { station }\end{array}$ & $\begin{array}{c}\text { Drainage } \\
\text { area } \\
\left(\mathrm{mi}^{2}\right)\end{array}$ & $\begin{array}{l}\text { Base flow } \\
\text { factor } \\
\left(\mathrm{ft}^{3} / \mathrm{s} / \mathrm{mi}^{2}\right)\end{array}$ & $\begin{array}{l}\text { Base flow } \\
\text { method }\end{array}$ & $\begin{array}{l}\text { Base flow } \\
\quad\left(\mathrm{ft}^{3} / \mathrm{s}\right)\end{array}$ & $\begin{array}{c}\text { Recharge } \\
\text { (inches) }\end{array}$ \\
\hline 99 & 040863075 & N Br Milwaukee R nr Random Lk & $43^{\circ} 33^{\prime} 25^{\prime \prime}$ & $88^{\circ} 03^{\prime} 10^{\prime \prime}$ & 04087000 & 51.4 & 0.3428 & equation & 28.9 & 7.6 \\
\hline 100 & 04086315 & Mink Ck nr Batavia & $43^{\circ} 33^{\prime} 28^{\prime \prime}$ & $88^{\circ} 03^{\prime} 20^{\prime \prime}$ & 04087000 & 19 & .2436 & equation & 8.8 & 6.3 \\
\hline 101 & 04086319 & Silver Ck at Random Lk & $43^{\circ} 33^{\prime} 26^{\prime \prime}$ & $87^{\circ} 58^{\prime} 27^{\prime \prime}$ & 04087000 & 11.7 & .0930 & relation & 2.9 & 3.4 \\
\hline 102 & 04086322 & Silver Ck nr Batavia & $43^{\circ} 33^{\prime} 25^{\prime \prime}$ & $88^{\circ} 02^{\prime} 15^{\prime \prime}$ & 04087000 & 18.7 & .0549 & equation & 4.0 & 2.9 \\
\hline 103 & 04086326 & N Br Milwaukee R nr Random Lk & $43^{\circ} 30^{\prime} 45^{\prime \prime}$ & $88^{\circ} 04^{\prime} 28^{\prime \prime}$ & 04087000 & 103.53 & .2394 & equation & 49.0 & 6.4 \\
\hline 104 & 04086330 & Stony $\mathrm{Ck}$ at Boltonville & $43^{\circ} 31^{\prime} 33^{\prime \prime}$ & $88^{\circ} 06^{\prime} 13^{\prime \prime}$ & 04087000 & 17.9 & .0605 & equation & 4.0 & 3.0 \\
\hline 105 & 04086350 & N Br Milwaukee R nr Fillmore & $43^{\circ} 28^{\prime} 45^{\prime \prime}$ & $88^{\circ} 02^{\prime} 05^{\prime \prime}$ & 04087000 & 149 & .1821 & equation & 61.6 & 5.6 \\
\hline 106 & 04086404 & Milwaukee R at Saukville & $43^{\circ} 22^{\prime} 55^{\prime \prime}$ & $87^{\circ} 57^{\prime} 15^{\prime \prime}$ & 04087000 & 457 & -- & relation & 203.0 & 6.0 \\
\hline 107 & 04086405 & Milwaukee R at Saukville & $43^{\circ} 22^{\prime} 31^{\prime \prime}$ & $87^{\circ} 57^{\prime} 34^{\prime \prime}$ & 04086500 & 461 & .2021 & equation & 205.8 & 6.1 \\
\hline 108 & 04086409 & Milwaukee R at Grafton & $43^{\circ} 20^{\prime} 11^{\prime \prime}$ & $87^{\circ} 56^{\prime} 57^{\prime \prime}$ & 04087000 & 8.53 & .2586 & equation & 4.0 & 6.4 \\
\hline 109 & 04086415 & Milwaukee R at Grafton & $43^{\circ} 18^{\prime} 27^{\prime \prime}$ & $87^{\circ} 57^{\prime} 08^{\prime \prime}$ & 04087000 & 476 & .1967 & equation & 209.7 & 6.0 \\
\hline 110 & 04086420 & Cedar Cr nr W Bend & $43^{\circ} 22^{\prime} 41^{\prime \prime}$ & $88^{\circ} 14^{\prime} 47^{\prime \prime}$ & 04086500 & 10.3 & .1611 & equation & 3.8 & 5.0 \\
\hline 111 & 04086425 & Cedar Cr nr Slinger & $43^{\circ} 21^{\prime} 13^{\prime \prime}$ & $88^{\circ} 14^{\prime} 01^{\prime \prime}$ & 04086500 & 13.9 & .0759 & equation & 3.5 & 3.4 \\
\hline 112 & 04086450 & Cedar Cr Tr \#3 nr Rockfield & $43^{\circ} 16^{\prime} 50^{\prime \prime}$ & $88^{\circ} 09^{\prime} 29^{\prime \prime}$ & 04086500 & 10.7 & .3680 & equation & 6.1 & 7.7 \\
\hline 113 & 04086460 & Cedar Cr nr Jackson & $43^{\circ} 19^{\prime} 25^{\prime \prime}$ & $88^{\circ} 08^{\prime} 32^{\prime \prime}$ & 04086500 & 53.6 & .2930 & relation & 36.0 & 9.1 \\
\hline 114 & 04086465 & Cedar Cr Tr nr Jackson & $43^{\circ} 20^{\prime} 25^{\prime \prime}$ & $88^{\circ} 09^{\prime} 41^{\prime \prime}$ & 04086500 & 4.06 & .0824 & equation & 1.0 & 3.5 \\
\hline 115 & 04086475 & Cedar Ck nr Jackson & $43^{\circ} 20^{\prime} 40^{\prime \prime}$ & $88^{\circ} 04^{\prime} 39^{\prime \prime}$ & 04086500 & 84.3 & .1755 & equation & 33.8 & 5.4 \\
\hline 116 & 04086478 & Cedar Ck Tr nr Newburg & $43^{\circ} 24^{\prime} 23^{\prime \prime}$ & $88^{\circ} 02^{\prime} 26^{\prime \prime}$ & 04086500 & 2.58 & .1845 & equation & 1.0 & 5.2 \\
\hline 117 & 04086485 & Cedar Ck Tr nr Jackson & $43^{\circ} 21^{\prime} 05^{\prime \prime}$ & $88^{\circ} 04^{\prime} 28^{\prime \prime}$ & 04086500 & 13.8 & .0965 & equation & 3.9 & 3.8 \\
\hline 118 & 04086700 & Pigeon $\mathrm{Ck}$ at Mequon & $43^{\circ} 13^{\prime} 57^{\prime \prime}$ & $87^{\circ} 59^{\prime} 01^{\prime \prime}$ & 04087000 & 11.6 & .0399 & equation & 2.1 & 2.4 \\
\hline 119 & 04086800 & Milwaukee $\mathrm{R}$ at Brown Deer & $43^{\circ} 10^{\prime} 39^{\prime \prime}$ & $87^{\circ} 57^{\prime} 20^{\prime \prime}$ & 04087000 & 656 & .1627 & equation & 263.5 & 5.5 \\
\hline 120 & 04087020 & $\begin{array}{l}\text { Menomonee } \mathrm{R} \text { at Menomonee } \\
\text { Falls }\end{array}$ & $43^{\circ} 11^{\prime} 32^{\prime \prime}$ & $88^{\circ} 07^{\prime} 58^{\prime \prime}$ & 04087120 & 30.7 & .1530 & relation & 10.7 & 4.7 \\
\hline 121 & 04087040 & Menomonee $\mathrm{R}$ at Butler & $43^{\circ} 06^{\prime} 51^{\prime \prime}$ & $88^{\circ} 03^{\prime} 56^{\prime \prime}$ & 04087120 & 60.6 & .1420 & relation & 18.0 & 4.0 \\
\hline 122 & 040872162 & Tr To Root R Tr nr Muskego & $42^{\circ} 55^{\prime} 25^{\prime \prime}$ & $88^{\circ} 05^{\prime} 46^{\prime \prime}$ & 04087240 & 3.07 & .0108 & equation & .3 & 1.2 \\
\hline
\end{tabular}

${ }^{1}$ Low-flow discharge measurements at partial-record stations were compared to one or more nearby USGS gaging stations to establish a relation between measured discharge and concurrent daily discharge. The nearby gaging station that provided the best relation with the partial-record station was chosen as the index station and was associated with nearby miscellaneous measurement sites.

${ }^{2}$ Mismatch between surface-water and ground-water drainage area. 
Appendix 9D. Average annual base flow and corresponding recharge, 1970-99, for partial-record stations in the Lake Michigan Basin-Continued.

[USGS, U.S. Geological Survey; ID, identification number; $\mathrm{dd}^{\circ} \mathrm{mm}$ 'ss", degrees, minutes, seconds; $\mathrm{mi}^{2}$, square mile; $\mathrm{ft}^{3} / \mathrm{s} / \mathrm{mi}^{2}$, cubic foot per second per square mile; $\mathrm{ft}^{3} / \mathrm{s}$, cubic foot per second; equation, statewide regression equation; relation, established relation line with index station; R, River; Bk, Brook; Br, Branch; Fk, Fork; Ck, Creek; Tr, Tributary; nr, near; N, North; S, South; E, East; W, West; ,-- not determined in historical low-flow reports. See references for full list of low-flow reports.]

\begin{tabular}{|c|c|c|c|c|c|c|c|c|c|c|}
\hline $\begin{array}{c}\text { Map } \\
\text { number }\end{array}$ & $\begin{array}{l}\text { USGS } \\
\text { station ID }\end{array}$ & Station name & $\begin{array}{c}\text { Latitude } \\
\text { (dd }{ }^{\circ} \mathrm{mm} \text { 'ss") }\end{array}$ & $\begin{array}{l}\text { Longitude } \\
\text { (dd }{ }^{\circ} \mathrm{mm} \text { 'ss") }\end{array}$ & $\begin{array}{l}\text { Index } \\
\text { station }{ }^{1}\end{array}$ & $\begin{array}{c}\text { Drainage } \\
\text { area } \\
\left(\mathrm{mi}^{2}\right)\end{array}$ & $\begin{array}{c}\text { Base flow } \\
\text { factor } \\
\left(\mathrm{ft}^{3} / \mathrm{s} / \mathrm{mi}^{2}\right)\end{array}$ & $\begin{array}{l}\text { Base flow } \\
\text { method }\end{array}$ & $\begin{array}{l}\text { Base flow } \\
\qquad\left(\mathrm{ft}^{3} / \mathrm{s}\right)\end{array}$ & $\begin{array}{c}\text { Recharge } \\
\text { (inches) }\end{array}$ \\
\hline 123 & 04087224 & $\begin{array}{l}\text { E Branch Root R Canal nr } \\
\text { Sturtevant }\end{array}$ & $42^{\circ} 43^{\prime} 09^{\prime \prime}$ & $87^{\circ} 58^{\prime} 56^{\prime \prime}$ & 04087240 & 5.51 & 0.0196 & equation & .7 & 1.6 \\
\hline 124 & 04087227 & $\begin{array}{l}\text { W Br Root R Canal at Union } \\
\text { Grove }\end{array}$ & $42^{\circ} 41^{\prime} 23^{\prime \prime}$ & $88^{\circ} 01^{\prime} 56^{\prime \prime}$ & 04087240 & 3.94 & .0801 & equation & 1.0 & 3.4 \\
\hline 125 & 040872325 & Root R Canal nr Raymond & $42^{\circ} 48^{\prime} 02^{\prime \prime}$ & $87^{\circ} 59^{\prime} 32^{\prime \prime}$ & 04087240 & 54.8 & .0788 & equation & 14.4 & 3.6 \\
\hline 126 & 04087235 & Root R nr Caledonia & $42^{\circ} 50^{\prime} 30^{\prime \prime}$ & $87^{\circ} 54^{\prime} 50^{\prime \prime}$ & 04087240 & 150 & .0310 & relation & 34.0 & 3.1 \\
\hline 127 & 04087238 & Hoods Ck nr Sturtevant & $42^{\circ} 43^{\prime} 14^{\prime \prime}$ & $87^{\circ} 55^{\prime} 23^{\prime \prime}$ & 04087240 & 3.38 & .0089 & equation & .3 & 1.1 \\
\hline 128 & 040872383 & Hoods Ck nr Sturtevant & $42^{\circ} 44^{\prime} 19^{\prime \prime}$ & $87^{\circ} 54^{\prime} 40^{\prime \prime}$ & 04087240 & 8.84 & .0085 & equation & .7 & 1.1 \\
\hline 129 & 04087248 & Pike R nr Sturtevant & $42^{\circ} 39^{\prime} 38^{\prime \prime}$ & $87^{\circ} 52^{\prime} 23^{\prime \prime}$ & 04087257 & 17.9 & .3422 & equation & 9.8 & 7.5 \\
\hline 130 & 04087251 & Pike Ck Tr nr Somers & $42^{\circ} 37^{\prime} 16^{\prime \prime}$ & $87^{\circ} 53^{\prime} 42^{\prime \prime}$ & 04087257 & 2.09 & .0228 & equation & .3 & 1.8 \\
\hline 131 & 04087252 & Pike Ck nr Somers & $42^{\circ} 38^{\prime} 23^{\prime \prime}$ & $87^{\circ} 52^{\prime} 54^{\prime \prime}$ & 04087257 & 13.08 & .0219 & equation & 1.7 & 1.8 \\
\hline 132 & 04087254 & Pike Ck Tr nr Somers & $42^{\circ} 38^{\prime} 39^{\prime \prime}$ & $87^{\circ} 52^{\prime} 54^{\prime \prime}$ & 04087257 & 2.71 & .0384 & equation & .5 & 2.3 \\
\hline 133 & 040872635 & Pike R nr Kenosha & $42^{\circ} 39^{\prime} 11^{\prime \prime}$ & $87^{\circ} 49^{\prime} 24^{\prime \prime}$ & 04087257 & 45.9 & .1613 & equation & 17.4 & 5.1 \\
\hline 134 & 04087269 & Pike Ck at Kenosha' & $42^{\circ} 35^{\prime} 58^{\prime \prime}$ & $87^{\circ} 50^{\prime} 25^{\prime \prime}$ & 04087257 & 2.37 & .1073 & equation & .7 & 3.9 \\
\hline
\end{tabular}

${ }^{1}$ Low-flow discharge measurements at partial-record stations were compared to one or more nearby USGS gaging stations to establish a relation between measured discharge and concurrent daily discharge. The nearby gaging station that provided the best relation with the partial-record station was chosen as the index station and was associated with nearby miscellaneous measurement sites.

${ }^{2}$ Mismatch between surface-water and ground-water drainage area. 

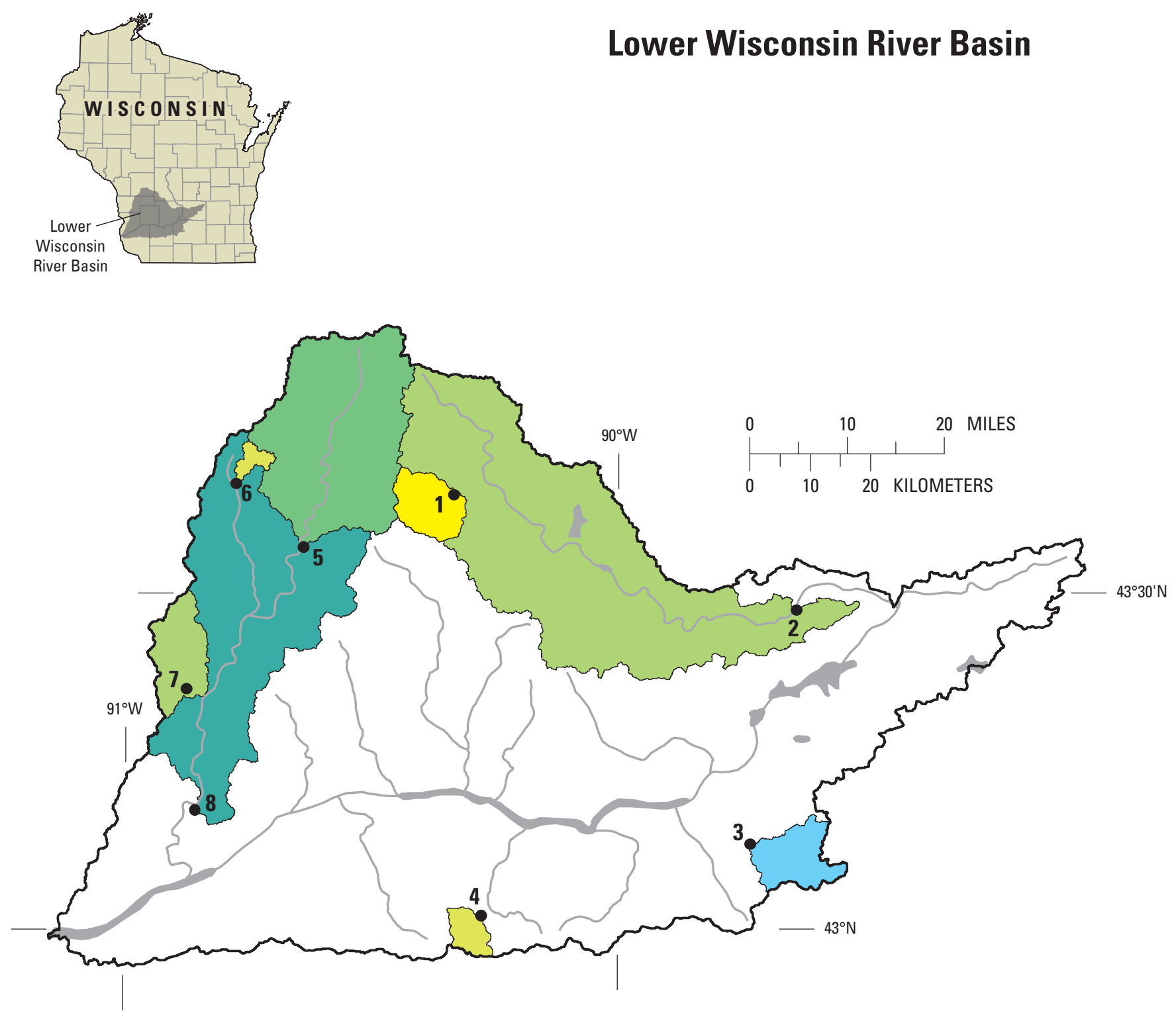

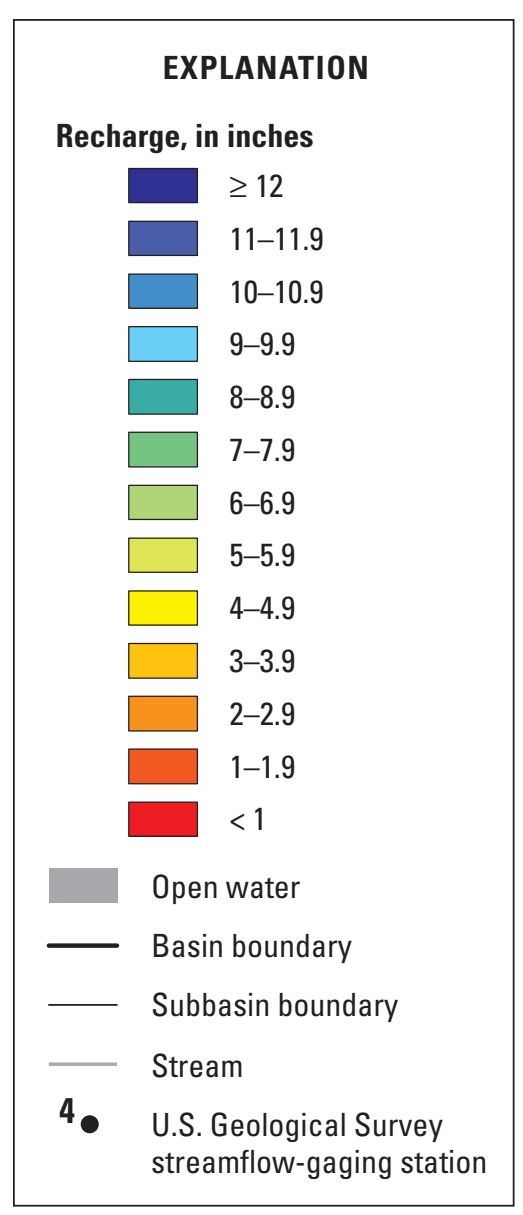




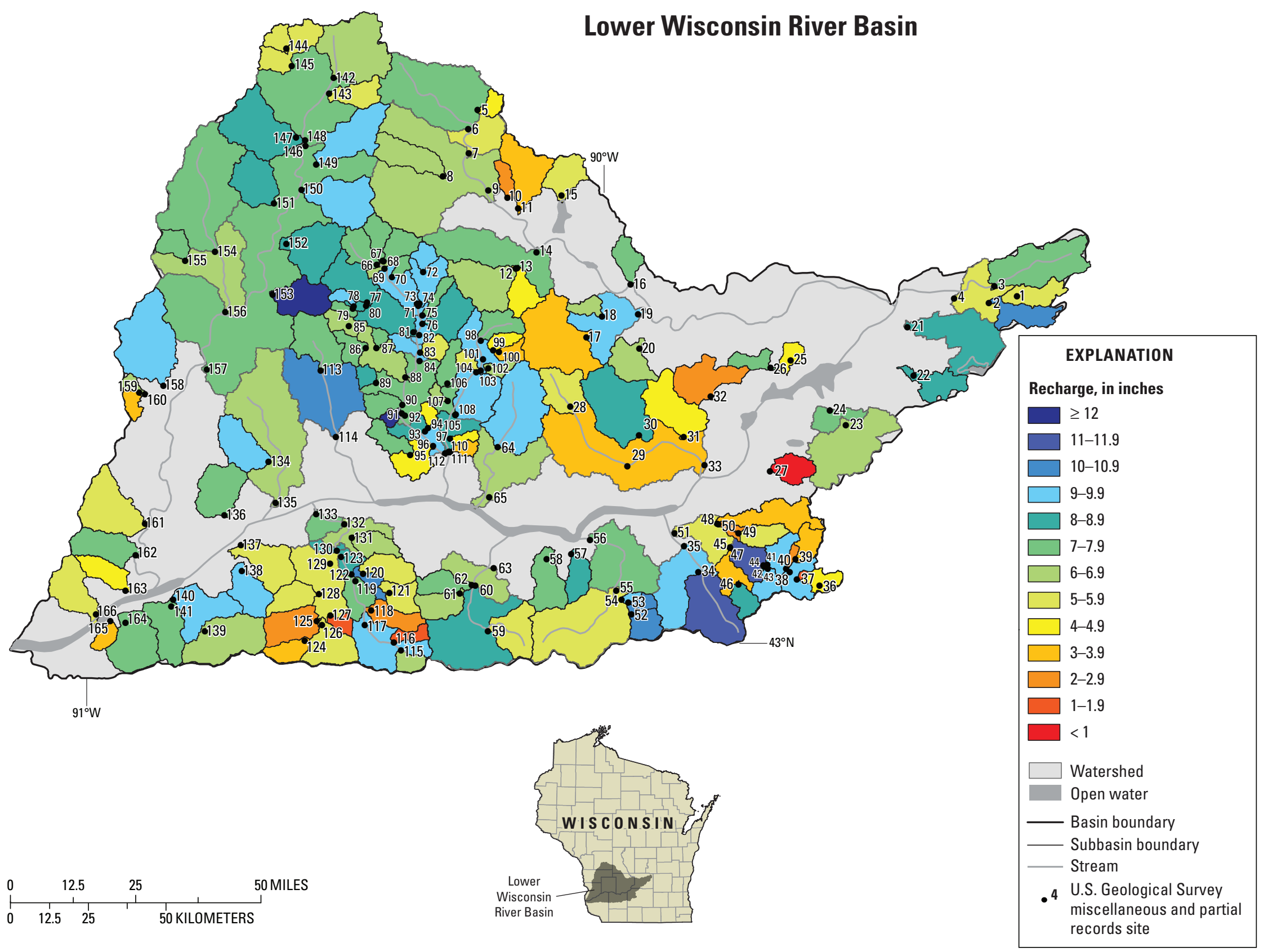


Appendix 10C. Average annual base flow and corresponding recharge, 1970-99, for streamflow-gaging stations in the Lower Wisconsin River Basin.

[USGS, U.S. Geological Survey; ID, identification number; mi² $^{2}$ square mile; ft³/s, cubic foot per second; BFI, Baseflow Index; ex rec, extended record; R, River; Bk, Brook; Br, Branch; Fk, Fork; Ck, Creek; Tr, Tributary; nr, near; N, North; S, South; E, East; W, West; --, not determined in historical low-flow reports. See references for full list of low-flow reports.]

\begin{tabular}{|c|c|c|c|c|c|c|c|c|c|c|c|}
\hline $\begin{array}{c}\text { Map } \\
\text { number }\end{array}$ & $\begin{array}{l}\text { USGS } \\
\text { station } \\
\text { ID }\end{array}$ & Station name & $\begin{array}{c}\text { Drainage } \\
\text { area } \\
\left(\mathrm{mi}^{2}\right)\end{array}$ & $\begin{array}{c}\text { Period of } \\
\text { analysis } \\
\text { (water years }{ }^{1} \text { ) }\end{array}$ & $\begin{array}{c}\text { Base flow } \\
\text { method }\end{array}$ & $\begin{array}{l}\text { Base } \\
\text { flow } \\
\text { index }\end{array}$ & $\begin{array}{c}\text { Base flow } \\
\quad\left(\mathrm{ft}^{3} / \mathrm{s}\right)\end{array}$ & $\begin{array}{c}\text { Recharge } \\
\text { (inches) }\end{array}$ & $\begin{array}{c}\text { Total } \\
\text { streamflow } \\
\left(\mathrm{ft}^{3} / \mathrm{s}\right)\end{array}$ & $\begin{array}{c}\text { Total } \\
\text { streamflow } \\
\text { (inches) }\end{array}$ & $\begin{array}{r}2090 \\
\left(\mathrm{ft}^{3} / \mathrm{s}\right)\end{array}$ \\
\hline 1 & 05404116 & S Br Baraboo R at Hillsboro & 39.1 & 1989-1999 & ex rec & -- & 12 & 4.1 & -- & -- & 66 \\
\hline 2 & 05405000 & Baraboo R nr Baraboo & 609 & 1970-1999 & BFI & 0.6400 & 270 & 6.0 & 430 & 9.56 & 170 \\
\hline 3 & 05406500 & Black Earth Ck at Black Earth & 45.6 & 1970-1999 & BFI & .8420 & 32 & 9.5 & 38 & 11.3 & 23 \\
\hline 4 & 05406640 & Otter Ck nr Highland & 16.8 & $1971-1975$ & ex rec & -- & 6.5 & 5.3 & -- & -- & -- \\
\hline 5 & 05408000 & Kickapoo R at La Farge & 266 & 1970-1999 & BFI & .7540 & 140 & 7.4 & 190 & 9.9 & 110 \\
\hline 6 & 05408500 & Knapp Ck nr Bloomingdale & 8.44 & 1955-1969 & ex rec & -- & 3.3 & 5.3 & -- & -- & 2.3 \\
\hline 7 & 05409890 & Nederlo Ck nr Gays Mills & 9.46 & $\begin{array}{c}1935-1960 \\
1979-1981\end{array}$ & ex rec & -- & 4.6 & 6.6 & -- & -- & 3.8 \\
\hline 8 & 05410490 & Kickapoo R at Steuben & 687 & 1970-1999 & BFI & .8430 & 454 & 9.0 & 540 & 10.7 & 320 \\
\hline
\end{tabular}

Water year is a 12-month period from October 1 through September 30, and is designated by the calendar year in which it ends (for example, the 2004 water year occurred October 1, 2003 through September 30, 2004).

${ }^{2}$ Discharge that is exceeded 90 percent of the time. 
Appendix 10D. Average annual base flow and corresponding recharge, 1970-99, for partial-record stations in the Lower Wisconsin River Basin.

[USGS, U.S. Geological Survey; ID, identification number; $\mathrm{dd}^{\circ} \mathrm{mm}$ 'ss", degrees, minutes, seconds; $\mathrm{mi}^{2}$, square mile; $\mathrm{ft}^{3} / \mathrm{s} / \mathrm{mi}^{2}$, cubic foot per second per square mile; $\mathrm{ft}^{3} / \mathrm{s}$, cubic foot per second; equation, statewide regression equation; relation, established relation line with index station; R, River; Bk, Brook; Br, Branch; Fk, Fork; Ck, Creek; Tr, Tributary; nr, near; N, North; S, South; E, East; W, West; ,-- not determined in historical low-flow reports. See references for full list of low-flow reports.]

\begin{tabular}{|c|c|c|c|c|c|c|c|c|c|c|}
\hline $\begin{array}{c}\text { Map } \\
\text { number }\end{array}$ & $\begin{array}{c}\text { USGS } \\
\text { station ID }\end{array}$ & Station name & $\begin{array}{c}\text { Latitude } \\
\text { (dd }{ }^{\circ} \mathrm{mm} \text { 'ss") }\end{array}$ & $\begin{array}{l}\text { Longitude } \\
\text { (dd }{ }^{\circ} \mathbf{m m} \mathbf{s s}^{\prime \prime)}\end{array}$ & $\begin{array}{c}\text { Index } \\
\text { station }^{1}\end{array}$ & $\begin{array}{c}\text { Drainage } \\
\text { area } \\
\left(\mathrm{mi}^{2}\right)\end{array}$ & $\begin{array}{c}\text { Base flow } \\
\text { factor } \\
\left(\mathrm{ft}^{3} / \mathrm{s} / \mathrm{mi}^{2}\right)\end{array}$ & $\begin{array}{c}\text { Base flow } \\
\text { method }\end{array}$ & $\begin{array}{c}\text { Base flow } \\
\quad\left(\mathrm{ft}^{3} / \mathbf{s}\right)\end{array}$ & $\begin{array}{c}\text { Recharge } \\
\text { (inches) }\end{array}$ \\
\hline 1 & 05404025 & Middle Br Duck Ck nr Rio & $43^{\circ} 29^{\prime} 54^{\prime \prime}$ & $89^{\circ} 11^{\prime} 30^{\prime \prime}$ & 05405000 & 7.72 & 0.1795 & equation & 3.0 & 5.3 \\
\hline 2 & 05404027 & Middle Br Duck Ck nr Rio & $43^{\circ} 29^{\prime} 20^{\prime \prime}$ & $89^{\circ} 14^{\prime} 47^{\prime \prime}$ & 05405000 & 28 & .4963 & equation & 18.9 & 9.1 \\
\hline 3 & 05404030 & N Br Duck Ck nr Pardeeville & $43^{\circ} 30^{\prime} 47^{\prime \prime}$ & $89^{\circ} 14^{\prime} 10^{\prime \prime}$ & 05405000 & 33.6 & .5220 & relation & 18.5 & 7.5 \\
\hline 4 & 05404033 & Duck Ck at Wyocena & $43^{\circ} 29^{\prime} 45^{\prime \prime}$ & $89^{\circ} 18^{\prime} 54^{\prime \prime}$ & 05405000 & 79.6 & .2220 & relation & 32.2 & 5.5 \\
\hline 5 & 05404050 & Cleaver Ck Tr nr Elroy & $43^{\circ} 46^{\prime} 00^{\prime \prime}$ & $90^{\circ} 14^{\prime} 57^{\prime \prime}$ & 05405000 & 4.16 & .1166 & equation & 1.3 & 4.1 \\
\hline 6 & 05404080 & Baraboo R at Elroy & $43^{\circ} 44^{\prime} 23^{\prime \prime}$ & $90^{\circ} 16^{\prime} 02^{\prime \prime}$ & 05405000 & 59.4 & .2935 & equation & 30.9 & 7.1 \\
\hline 7 & 05404100 & Baraboo R nr Union Center & $43^{\circ} 42^{\prime} 18^{\prime \prime}$ & $90^{\circ} 15^{\prime} 59^{\prime \prime}$ & 05405000 & 73.1 & .2870 & relation & 29.0 & 5.4 \\
\hline 8 & 05404118 & Hills Ck nr Hillsboro & $43^{\circ} 40^{\prime} 19^{\prime \prime}$ & $90^{\circ} 19^{\prime} 02^{\prime \prime}$ & 05405000 & 16.2 & .2612 & equation & 7.7 & 6.5 \\
\hline 9 & 05404123 & Baraboo $\mathrm{R}$ at Wonewok & $43^{\circ} 39^{\prime} 08^{\prime \prime}$ & $90^{\circ} 13^{\prime} 40^{\prime \prime}$ & 05405000 & 174 & .2702 & equation & 88.6 & 6.9 \\
\hline 10 & 05404125 & Gardner $\mathrm{Ck}$ at Wonewoc & $43^{\circ} 38^{\prime} 32^{\prime \prime}$ & $90^{\circ} 11^{\prime} 24^{\prime \prime}$ & 05405000 & 4.45 & .0623 & equation & 1.0 & 3.0 \\
\hline 11 & 05404127 & Crossman Ck nr Lavalle & $43^{\circ} 37^{\prime} 36^{\prime \prime}$ & $90^{\circ} 10^{\prime} 08^{\prime \prime}$ & 05405000 & 19.9 & .1018 & equation & 5.8 & 4.0 \\
\hline 12 & 05404133 & Mcglynn Ck nr Cazenovia & $43^{\circ} 32^{\prime} 24^{\prime \prime}$ & $90^{\circ} 10^{\prime} 23^{\prime \prime}$ & 05405000 & 23.9 & .2624 & equation & 11.5 & 6.5 \\
\hline 13 & 05404134 & Mcglynn Ck nr Ironton & $43^{\circ} 32^{\prime} 27^{\prime \prime}$ & $90^{\circ} 10^{\prime} 12^{\prime \prime}$ & 05405000 & 33.5 & .1169 & equation & 10.7 & 4.3 \\
\hline 14 & 05404137 & Little Baraboo R nr Lavalle & $43^{\circ} 33^{\prime} 50^{\prime \prime}$ & $90^{\circ} 07^{\prime} 59^{\prime \prime}$ & 05405000 & 69 & .2099 & equation & 30.2 & 6.0 \\
\hline 15 & 05404140 & W Br Big Ck nr La Valle & $43^{\circ} 38^{\prime} 41^{\prime \prime}$ & $90^{\circ} 05^{\prime} 01^{\prime \prime}$ & 05405000 & 11.9 & .1718 & equation & 4.5 & 5.2 \\
\hline 16 & 05404175 & Copper Ck nr Reedsburg & $43^{\circ} 31^{\prime} 05^{\prime \prime}$ & $89^{\circ} 56^{\prime} 53^{\prime \prime}$ & 05405000 & 8.25 & .3500 & equation & 4.5 & 7.4 \\
\hline 17 & 05404200 & Narrows $\mathrm{Ck}$ at Loganville & $43^{\circ} 26^{\prime} 32^{\prime \prime}$ & $90^{\circ} 02^{\prime} 06^{\prime \prime}$ & 05405000 & 40.1 & .1440 & relation & 11.5 & 3.9 \\
\hline 18 & 05404205 & Narrows Ck Tr nr Rock Springs & $43^{\circ} 28^{\prime} 20^{\prime \prime}$ & $90^{\circ} 00^{\prime} 14^{\prime \prime}$ & 05405000 & 4.57 & .2402 & equation & 2.0 & 6.0 \\
\hline 19 & 05404250 & Narrows Ck nr Rock Springs & $43^{\circ} 28^{\prime} 31^{\prime \prime}$ & $89^{\circ} 56^{\prime} 02^{\prime \prime}$ & 05405000 & 68.1 & .2000 & equation & 29.1 & 5.8 \\
\hline 20 & 05404400 & Seeley Ck nr N Freedom & $43^{\circ} 25^{\prime} 37^{\prime \prime}$ & $89^{\circ} 55^{\prime} 51^{\prime \prime}$ & 05405000 & 12.4 & .2518 & equation & 5.8 & 6.3 \\
\hline 21 & 05405560 & Rocky Run nr Poynette & $43^{\circ} 27^{\prime} 21^{\prime \prime}$ & $89^{\circ} 24^{\prime} 24^{\prime \prime}$ & 05405000 & 32.9 & .4129 & equation & 20.2 & 8.3 \\
\hline 22 & 05405600 & Rowan $\mathrm{Ck}$ at Poynette & $43^{\circ} 23^{\prime} 11^{\prime \prime}$ & $89^{\circ} 23^{\prime} 43^{\prime \prime}$ & 05406500 & 10.4 & .5030 & relation & 6.8 & 8.9 \\
\hline 23 & 05405800 & Spring $\mathrm{Ck}$ at Lodi & $43^{\circ} 19^{\prime} 00^{\prime \prime}$ & $89^{\circ} 31^{\prime} 40^{\prime \prime}$ & 05405000 & 37 & .5750 & relation & 18.0 & 6.6 \\
\hline 24 & 05405812 & Spring Ck nr Lodi & $43^{\circ} 20^{\prime} 14^{\prime \prime}$ & $89^{\circ} 33^{\prime} 34^{\prime \prime}$ & 05405000 & 45.5 & .3094 & equation & 24.2 & 7.2 \\
\hline 25 & 05405820 & Wisconsin R Tr nr Merrimac & $43^{\circ} 24^{\prime} 33^{\prime \prime}$ & $89^{\circ} 38^{\prime} 08^{\prime \prime}$ & 05405000 & 2.82 & .1224 & equation & 0.9 & 4.2 \\
\hline 26 & 05405850 & Wisconsin R Tr nr Merrimac & $43^{\circ} 23^{\prime} 56^{\prime \prime}$ & $89^{\circ} 40^{\prime} 30^{\prime \prime}$ & 05405000 & 3.16 & .3390 & equation & 1.7 & 7.2 \\
\hline
\end{tabular}

${ }^{1}$ Low-flow discharge measurements at partial-record stations were compared to one or more nearby USGS gaging stations to establish a relation between measured discharge and concurrent daily discharge. The nearby gaging station that provided the best relation with the partial-record station was chosen as the index station and was associated with nearby miscellaneous measurement sites.

${ }^{2}$ Mismatch between surface-water and ground-water drainage area. 
Appendix 10D. Average annual base flow and corresponding recharge, 1970-99, for partial-record stations in the Lower Wisconsin River Basin-Continued.

[USGS, U.S. Geological Survey; ID, identification number; $\mathrm{dd}^{\circ} \mathrm{mm}$ 'ss", degrees, minutes, seconds; $\mathrm{mi}^{2}$, square mile; $\mathrm{ft}^{3} / \mathrm{s} / \mathrm{mi}^{2}$, cubic foot per second per square mile; $\mathrm{ft}^{3} / \mathrm{s}$, cubic foot per second; equation, statewide regression equation; relation, established relation line with index station; R, River; Bk, Brook; Br, Branch; Fk, Fork; Ck, Creek; Tr, Tributary; nr, near; N, North; S, South; E, East; W, West; --, not determined in historical low-flow reports. See references for full list of low-flow reports.]

\begin{tabular}{|c|c|c|c|c|c|c|c|c|c|c|}
\hline $\begin{array}{l}\text { Map } \\
\text { number }\end{array}$ & $\begin{array}{c}\text { USGS } \\
\text { station ID }\end{array}$ & Station name & $\begin{array}{c}\text { Latitude } \\
\text { (dd }{ }^{\circ} \mathrm{mm} \text { 'ss") }\end{array}$ & $\begin{array}{l}\text { Longitude } \\
\text { (ddºmm'ss") }\end{array}$ & $\begin{array}{l}\text { Index } \\
\text { station }{ }^{1}\end{array}$ & $\begin{array}{c}\text { Drainage } \\
\text { area } \\
\left(\mathrm{mi}^{2}\right)\end{array}$ & $\begin{array}{c}\text { Base flow } \\
\text { factor } \\
\left(\mathrm{ft}^{3} / \mathrm{s} / \mathrm{mi}^{2}\right)\end{array}$ & $\begin{array}{l}\text { Base flow } \\
\text { method }\end{array}$ & $\begin{array}{l}\text { Base flow } \\
\quad\left(\mathrm{ft}^{3} / \mathrm{s}\right)\end{array}$ & $\begin{array}{c}\text { Recharge } \\
\text { (inches) }\end{array}$ \\
\hline 27 & 05406060 & Blum'S Ck at Roxbury & $43^{\circ} 15^{\prime} 06^{\prime \prime}$ & $89^{\circ} 40^{\prime} 34^{\prime \prime}$ & 05406500 & 9.27 & 0.0035 & equation & .5 & .7 \\
\hline 28 & 05406110 & Honey Ck nr Plain & $43^{\circ} 20^{\prime} 38^{\prime \prime}$ & $90^{\circ} 03^{\prime} 58^{\prime \prime}$ & 05405000 & 14 & .1807 & equation & 5.5 & 5.3 \\
\hline 29 & 05406200 & Honey Creek Nr Plain & $43^{\circ} 15^{\prime} 31^{\prime \prime}$ & $89^{\circ} 57^{\prime} 15^{\prime \prime}$ & 05406500 & 55.93 & .1630 & relation & 14.6 & 3.5 \\
\hline 30 & 05406220 & N Br Honey Ck nr Leland & $43^{\circ} 18^{\prime} 10^{\prime \prime}$ & $89^{\circ} 55^{\prime} 55^{\prime \prime}$ & 05405000 & 35.3 & .3902 & equation & 21.1 & 8.1 \\
\hline 31 & 05406230 & E Br Honey Ck nr Witwen & $43^{\circ} 18^{\prime} 02^{\prime \prime}$ & $89^{\circ} 50^{\prime} 41^{\prime \prime}$ & 05405000 & 23 & .1529 & equation & 8.4 & 4.9 \\
\hline 32 & 05406240 & Otter Ck nr Prairie Du Sac & $43^{\circ} 21^{\prime} 30^{\prime \prime}$ & $89^{\circ} 47^{\prime} 30^{\prime \prime}$ & 05405000 & 17.9 & .0339 & equation & 3.0 & 2.2 \\
\hline 33 & 05406300 & Honey Ck nr Sauk City & $43^{\circ} 15^{\prime} 35^{\prime \prime}$ & $89^{\circ} 48^{\prime} 16^{\prime \prime}$ & 05405000 & 187 & .2350 & relation & 62.3 & 4.5 \\
\hline 34 & 05406400 & $\begin{array}{l}\text { E Br Blue Mounds Ck nr Black } \\
\text { Earth }\end{array}$ & $43^{\circ} 06^{\prime} 30^{\prime \prime}$ & $89^{\circ} 49^{\prime} 00^{\prime \prime}$ & 05406500 & 29 & .6790 & relation & 24.1 & 11.3 \\
\hline 35 & 05406430 & Blue Mounds Ck nr Mazomanie & $43^{\circ} 08^{\prime} 41^{\prime \prime}$ & $89^{\circ} 50^{\prime} 40^{\prime \prime}$ & 05406500 & 55 & .5707 & equation & 40.4 & 10.0 \\
\hline 36 & 05406450 & Black Earth Ck nr Cross Plains & $43^{\circ} 05^{\prime} 16^{\prime \prime}$ & $89^{\circ} 34^{\prime} 52^{\prime \prime}$ & 05406500 & 3.32 & .1428 & equation & 1.1 & 4.6 \\
\hline 37 & 054064509 & Black Earth Ck nr Cross Plains & $43^{\circ} 05^{\prime} 49^{\prime \prime}$ & $89^{\circ} 37^{\prime} 32^{\prime \prime}$ & 05406500 & 9.53 & -- & relation & 1.2 & ${ }^{2} 1.7$ \\
\hline 38 & 05406455 & Black Earth Ck at Cross Plains & $43^{\circ} 06^{\prime} 25^{\prime \prime}$ & $89^{\circ} 38^{\prime} 22^{\prime \prime}$ & 05406500 & 11.3 & .5464 & equation & 7.9 & 9.4 \\
\hline 39 & 054064685 & Brewery Cr nr Cross Plains & $43^{\circ} 07^{\prime} 33^{\prime \prime}$ & $89^{\circ} 37^{\prime} 40^{\prime \prime}$ & 05406500 & 9.3 & .1061 & equation & 2.8 & 4.0 \\
\hline 40 & 05406475 & Brewery Cr nr Cross Plains & $43^{\circ} 06^{\prime} 43^{\prime \prime}$ & $89^{\circ} 38^{\prime} 46^{\prime \prime}$ & 05406500 & 12.5 & .0571 & equation & 2.7 & 2.9 \\
\hline 41 & 05406487 & Black Earth Ck nr Cross Plains & $43^{\circ} 07^{\prime} 05^{\prime \prime}$ & $89^{\circ} 40^{\prime} 57^{\prime \prime}$ & 05406500 & 27 & .5833 & equation & 19.8 & 9.9 \\
\hline 42 & 05406489 & Black Earth Ck above Garfoot Ck & $43^{\circ} 06^{\prime} 56^{\prime \prime}$ & $89^{\circ} 41^{\prime} 18^{\prime \prime}$ & 05406500 & 33 & -- & relation & 22.5 & 9.3 \\
\hline 43 & 054064915 & Garfoot Ck nr Cross Plains & $43^{\circ} 06^{\prime} 43^{\prime \prime}$ & $89^{\circ} 40^{\prime} 54^{\prime \prime}$ & 05406500 & 3.91 & .6284 & equation & 2.9 & 9.9 \\
\hline 44 & 05406494 & Black Earth Ck nr Cross Plains & $43^{\circ} 07^{\prime} 02^{\prime \prime}$ & $89^{\circ} 41^{\prime} 24^{\prime \prime}$ & 05406500 & 39.1 & -- & relation & 24.7 & 8.6 \\
\hline 45 & 05406510 & Black Earth $\mathrm{Ck}$ atVermont $\mathrm{Ck}$ & $43^{\circ} 08^{\prime} 34^{\prime \prime}$ & $89^{\circ} 45^{\prime} 18^{\prime \prime}$ & 05406500 & 48.4 & .4909 & equation & 32.8 & 9.2 \\
\hline 46 & 05406513 & Vermont Cr nr Black Earth & $43^{\circ} 05^{\prime} 24^{\prime \prime}$ & $89^{\circ} 44^{\prime} 19^{\prime \prime}$ & 05406500 & 6.1 & .4338 & equation & 3.7 & 8.3 \\
\hline 47 & 05406515 & Vermont Ck at Mouth & $43^{\circ} 08^{\prime} 33^{\prime \prime}$ & $89^{\circ} 45^{\prime} 18^{\prime \prime}$ & 05406500 & 14.9 & -- & relation & 6.2 & 5.7 \\
\hline 48 & 05406524 & Halfway Prairie $\mathrm{Cr}$ at Mazomanie & $43^{\circ} 10^{\prime} 37^{\prime \prime}$ & $89^{\circ} 46^{\prime} 48^{\prime \prime}$ & 05406500 & 16.7 & -- & relation & 4.9 & 4.0 \\
\hline 49 & 05406527 & Marsh Valley Cr nr Black Earth & $43^{\circ} 09^{\prime} 45^{\prime \prime}$ & $89^{\circ} 44^{\prime} 19^{\prime \prime}$ & 05406500 & 8.1 & .2172 & equation & 3.5 & 5.9 \\
\hline 50 & 054065295 & Marsh Valley Cr at Mazomanie & $43^{\circ} 10^{\prime} 34^{\prime \prime}$ & $89^{\circ} 46^{\prime} 40^{\prime \prime}$ & 05406500 & 10.5 & -- & relation & 4.0 & 5.2 \\
\hline 51 & 05406548 & Black Earth Ck nr Mazomanie & $43^{\circ} 09^{\prime} 46^{\prime \prime}$ & $89^{\circ} 51^{\prime} 46^{\prime \prime}$ & 05406500 & 105 & -- & relation & 41.1 & 5.3 \\
\hline
\end{tabular}

${ }^{1}$ Low-flow discharge measurements at partial-record stations were compared to one or more nearby USGS gaging stations to establish a relation between measured discharge and concurrent daily discharge. The nearby gaging station that provided the best relation with the partial-record station was chosen as the index station and was associated with nearby miscellaneous measurement sites.

${ }^{2}$ Mismatch between surface-water and ground-water drainage area. 
[USGS, U.S. Geological Survey; ID, identification number; $\mathrm{dd}^{\circ} \mathrm{mm}$ 'ss", degrees, minutes, seconds; $\mathrm{mi}^{2}$, square mile; $\mathrm{ft}^{3} / \mathrm{s} / \mathrm{mi}^{2}$, cubic foot per second per square mile; $\mathrm{ft}^{3} / \mathrm{s}$, cubic foot per second; equation, statewide regression equation; relation, established relation line with index station; R, River; Bk, Brook; Br, Branch; Fk, Fork; Ck, Creek; Tr, Tributary; nr, near; N, North; S, South; E, East; W, West; --, not determined in historical low-flow reports. See references for full list of low-flow reports.]

\begin{tabular}{|c|c|c|c|c|c|c|c|c|c|c|}
\hline $\begin{array}{c}\text { Map } \\
\text { number }\end{array}$ & $\begin{array}{l}\text { USGS } \\
\text { station ID }\end{array}$ & Station name & $\begin{array}{c}\text { Latitude } \\
\text { (dd }{ }^{\circ} \mathrm{mm} \text { 'ss") }\end{array}$ & $\begin{array}{l}\text { Longitude } \\
\text { (dd }{ }^{\circ} \mathrm{mm} \text { 'ss") }\end{array}$ & $\begin{array}{l}\text { Index } \\
\text { station }{ }^{1}\end{array}$ & $\begin{array}{c}\text { Drainage } \\
\text { area } \\
\left(\mathrm{mi}^{2}\right) \\
\end{array}$ & $\begin{array}{c}\text { Base flow } \\
\text { factor } \\
\left(\mathrm{ft}^{3} / \mathrm{s} / \mathrm{mi}^{2}\right)\end{array}$ & $\begin{array}{l}\text { Base flow } \\
\text { method }\end{array}$ & $\begin{array}{c}\text { Base flow } \\
\quad\left(\mathrm{ft}^{3} / \mathrm{s}\right)\end{array}$ & $\begin{array}{c}\text { Recharge } \\
\text { (inches) }\end{array}$ \\
\hline 52 & 05406573 & Trout Ck nr Barneveld & $43^{\circ} 02^{\prime} 52^{\prime \prime}$ & $89^{\circ} 56^{\prime} 48^{\prime \prime}$ & 05406500 & 8.37 & -- & relation & 6.3 & 10.2 \\
\hline 53 & 05406575 & $\begin{array}{l}\text { Trout Ck at Co Hwy T nr } \\
\text { Barneveld }\end{array}$ & $43^{\circ} 03^{\prime} 52^{\prime \prime}$ & $89^{\circ} 57^{\prime} 10^{\prime \prime}$ & 05406500 & 12.1 & -- & relation & 9.3 & 10.4 \\
\hline 54 & 05406577 & Trout Ck nr Ridgeway & $43^{\circ} 04^{\prime} 08^{\prime \prime}$ & $89^{\circ} 57^{\prime} 57^{\prime \prime}$ & 05406500 & 13.5 & -- & relation & 9.6 & 9.7 \\
\hline 55 & 05406580 & Mill Ck nr Barneveld & $43^{\circ} 04^{\prime} 54^{\prime \prime}$ & $89^{\circ} 58^{\prime} 35^{\prime \prime}$ & 05406500 & 66.5 & 0.2084 & equation & 29.0 & 5.9 \\
\hline 56 & 05406600 & Mill Ck nr Spring Green & $43^{\circ} 09^{\prime} 15^{\prime \prime}$ & $90^{\circ} 01^{\prime} 38^{\prime \prime}$ & 05406500 & 96.8 & .5570 & relation & 56.2 & 7.9 \\
\hline 57 & 05406605 & Lowery Ck nr Spring Green & $43^{\circ} 08^{\prime} 00^{\prime \prime}$ & $90^{\circ} 03^{\prime} 52^{\prime \prime}$ & 05406500 & 8.76 & .4405 & equation & 5.4 & 8.4 \\
\hline 58 & 05406615 & Rush Ck nr Spring Green & $43^{\circ} 07^{\prime} 33^{\prime \prime}$ & $90^{\circ} 06^{\prime} 41^{\prime \prime}$ & 05406500 & 13.9 & -- & relation & 7.2 & 7.0 \\
\hline 59 & 05406643 & Flint Ck nr Clyde & $43^{\circ} 01^{\prime} 24^{\prime \prime}$ & $90^{\circ} 13^{\prime} 35^{\prime \prime}$ & 05406500 & 16.7 & .1871 & equation & 6.7 & 5.5 \\
\hline 60 & 05406646 & Otter Ck nr Highland & $43^{\circ} 05^{\prime} 18^{\prime \prime}$ & $90^{\circ} 15^{\prime} 03^{\prime \prime}$ & 05406500 & 60.5 & .4065 & equation & 37.3 & 8.4 \\
\hline 61 & 054066478 & Tr To Otter Ck Tr nr Highland & $43^{\circ} 04^{\prime} 36^{\prime \prime}$ & $90^{\circ} 16^{\prime} 50^{\prime \prime}$ & 05406500 & 10.3 & .2367 & equation & 4.6 & 6.1 \\
\hline 62 & 05406648 & Otter Ck Tr nr Highland & $43^{\circ} 05^{\prime} 21^{\prime \prime}$ & $90^{\circ} 15^{\prime} 26^{\prime \prime}$ & 05406500 & 19.8 & .3692 & equation & 11.4 & 7.8 \\
\hline 63 & 05406650 & Otter Ck nr Clyde & $43^{\circ} 06^{\prime} 48^{\prime \prime}$ & $90^{\circ} 12^{\prime} 53^{\prime \prime}$ & 05406500 & 95.6 & .3530 & relation & 49.0 & 7.0 \\
\hline 64 & 05406680 & Bear Ck nr Plain & $43^{\circ} 17^{\prime} 09^{\prime \prime}$ & $90^{\circ} 12^{\prime} 29^{\prime \prime}$ & 05406500 & 37.5 & .4964 & equation & 25.4 & 9.2 \\
\hline 65 & 05406700 & Bear Ck nr Lone Rock & $43^{\circ} 12^{\prime} 54^{\prime \prime}$ & $90^{\circ} 13^{\prime} 27^{\prime \prime}$ & 05406500 & 65.5 & .3400 & relation & 29.3 & 6.1 \\
\hline 66 & 05406704 & Pine R nr Yuba & $43^{\circ} 32^{\prime} 42^{\prime \prime}$ & $90^{\circ} 26^{\prime} 45^{\prime \prime}$ & 05408000 & 9.64 & .3521 & equation & 5.3 & 7.5 \\
\hline 67 & 05406706 & Pine R Tr nr Yuba & $43^{\circ} 33^{\prime} 03^{\prime \prime}$ & $90^{\circ} 26^{\prime} 03^{\prime \prime}$ & 05408000 & 7.37 & .3691 & equation & 4.1 & 7.6 \\
\hline 68 & 05406708 & Tr To Pine R Tr nr Yuba & $43^{\circ} 33^{\prime} 02^{\prime \prime}$ & $90^{\circ} 25^{\prime} 57^{\prime \prime}$ & 05408000 & 8.13 & .3493 & equation & 4.5 & 7.4 \\
\hline 69 & 05406710 & Pine $R$ at Yuba & $43^{\circ} 32^{\prime} 22^{\prime \prime}$ & $90^{\circ} 25^{\prime} 50^{\prime \prime}$ & 05408000 & 27.2 & -- & relation & 12.6 & 6.3 \\
\hline 70 & 05406711 & Indian Ck nr Yuba & $43^{\circ} 31^{\prime} 40^{\prime \prime}$ & $90^{\circ} 24^{\prime} 57^{\prime \prime}$ & 05408000 & 2.59 & .3308 & equation & 1.3 & 7.1 \\
\hline 71 & 05406714 & Pine R nr Hub City & $43^{\circ} 29^{\prime} 24^{\prime \prime}$ & $90^{\circ} 22^{\prime} 00^{\prime \prime}$ & 05408000 & 41.1 & .4987 & equation & 28.0 & 9.2 \\
\hline 72 & 05406716 & Melancthon Ck nr Yuba & $43^{\circ} 32^{\prime} 08^{\prime \prime}$ & $90^{\circ} 21^{\prime} 15^{\prime \prime}$ & 05408000 & 4.66 & .5505 & equation & 3.2 & 9.3 \\
\hline 73 & 05406717 & Melancthon Ck nr Hub City & $43^{\circ} 29^{\prime} 25^{\prime \prime}$ & $90^{\circ} 21^{\prime} 39^{\prime \prime}$ & 05408000 & 14.3 & .5225 & equation & 9.8 & 9.3 \\
\hline 74 & 05406718 & Pine R nr Hub City & $43^{\circ} 29^{\prime} 13^{\prime \prime}$ & $90^{\circ} 21^{\prime} 50^{\prime \prime}$ & 05408000 & 55.5 & .5159 & equation & 38.7 & 9.5 \\
\hline 75 & 05406719 & Soules Ck at Hub City & $43^{\circ} 28^{\prime} 25^{\prime \prime}$ & $90^{\circ} 21^{\prime} 20^{\prime \prime}$ & 05408000 & 4.4 & .3733 & equation & 2.5 & 7.6 \\
\hline 76 & 05406722 & Hawkins Ck at Hub City & $43^{\circ} 27^{\prime} 40^{\prime \prime}$ & $90^{\circ} 21^{\prime} 20^{\prime \prime}$ & 05408000 & 15.2 & .4852 & equation & 10.0 & 8.9 \\
\hline 77 & 05406732 & W Br Pine R nr Bloom City & $43^{\circ} 29^{\prime} 30^{\prime \prime}$ & $90^{\circ} 27^{\prime} 50^{\prime \prime}$ & 05408000 & 14.6 & .4353 & equation & 9.1 & 8.4 \\
\hline
\end{tabular}

${ }^{1}$ Low-flow discharge measurements at partial-record stations were compared to one or more nearby USGS gaging stations to establish a relation between measured discharge and concurrent daily discharge. The nearby gaging station that provided the best relation with the partial-record station was chosen as the index station and was associated with nearby miscellaneous measurement sites

${ }^{2}$ Mismatch between surface-water and ground-water drainage area.v 
Appendix 10D. Average annual base flow and corresponding recharge, 1970-99, for partial-record stations in the Lower Wisconsin River Basin-Continued.

[USGS, U.S. Geological Survey; ID, identification number; $\mathrm{dd}^{\circ} \mathrm{mm}$ 'ss", degrees, minutes, seconds; $\mathrm{mi}^{2}$, square mile; $\mathrm{ft}^{3} / \mathrm{s} / \mathrm{mi}^{2}$, cubic foot per second per square mile; $\mathrm{ft}^{3} / \mathrm{s}$, cubic foot per second; equation, statewide regression equation; relation, established relation line with index station; R, River; Bk, Brook; Br, Branch; Fk, Fork; Ck, Creek; Tr, Tributary; nr, near; N, North; S, South; E, East; W, West; ,-- not determined in historical low-flow reports. See references for full list of low-flow reports.]

\begin{tabular}{|c|c|c|c|c|c|c|c|c|c|c|}
\hline $\begin{array}{c}\text { Map } \\
\text { number }\end{array}$ & $\begin{array}{c}\text { USGS } \\
\text { station ID }\end{array}$ & Station name & $\begin{array}{c}\text { Latitude } \\
\text { (dd }{ }^{\circ} \mathbf{m m} \mathbf{s s}^{\prime \prime)}\end{array}$ & $\begin{array}{l}\text { Longitude } \\
\text { (ddºmm'ss") }\end{array}$ & $\begin{array}{l}\text { Index } \\
\text { station }{ }^{1}\end{array}$ & $\begin{array}{c}\text { Drainage } \\
\text { area } \\
\left(\mathrm{mi}^{2}\right)\end{array}$ & $\begin{array}{l}\text { Base flow } \\
\text { factor } \\
\left(\mathrm{ft}^{3} / \mathrm{s} / \mathrm{mi}^{2}\right)\end{array}$ & $\begin{array}{l}\text { Base flow } \\
\text { method }\end{array}$ & $\begin{array}{l}\text { Base flow } \\
\quad\left(\mathrm{ft}^{3} / \mathrm{s}\right)\end{array}$ & $\begin{array}{c}\text { Recharge } \\
\text { (inches) }\end{array}$ \\
\hline 78 & 05406735 & W Br Pine R Tr nr Bloom City & $43^{\circ} 29^{\prime} 10^{\prime \prime}$ & $90^{\circ} 29^{\prime 2} 24^{\prime \prime}$ & 05408000 & 2.47 & 0.5801 & equation & 1.7 & 9.5 \\
\hline 79 & 05406737 & $\begin{array}{l}\text { Tr To W Br Pine R Tr nr Bloom } \\
\text { City }\end{array}$ & $43^{\circ} 28^{\prime} 57^{\prime \prime}$ & $90^{\circ} 29^{\prime} 30^{\prime \prime}$ & 05408000 & 2.59 & .3728 & equation & 1.4 & 7.5 \\
\hline 80 & 05406740 & W Br Pine R Tr at Bloom City & $43^{\circ} 29^{\prime} 12^{\prime \prime}$ & $90^{\circ} 27^{\prime} 56^{\prime \prime}$ & 05408000 & 6.13 & .4881 & equation & 4.0 & 8.8 \\
\hline 81 & 05406743 & W Br Pine R nr Rockbridge & $43^{\circ} 26^{\prime} 58^{\prime \prime}$ & $90^{\circ} 22^{\prime} 22^{\prime \prime}$ & 05408000 & 37.6 & -- & relation & 23.1 & 8.3 \\
\hline 82 & 05406745 & Pine $\mathrm{R}$ at Rockbridge & $43^{\circ} 26^{\prime} 43^{\prime \prime}$ & $90^{\circ} 21^{\prime} 43^{\prime \prime}$ & 05408000 & 117 & -- & relation & 79.1 & 9.2 \\
\hline 83 & 05406748 & S Buck Ck at Buck Ck & $43^{\circ} 25^{\prime} 13^{\prime \prime}$ & $90^{\circ} 21 ' 37^{\prime \prime}$ & 05408000 & 4.95 & .2267 & equation & 2.1 & 5.9 \\
\hline 84 & 05406750 & Pine R nr Buck Ck & $43^{\circ} 24^{\prime} 30^{\prime \prime}$ & $90^{\circ} 21^{\prime} 40^{\prime \prime}$ & 05408000 & 128 & .5020 & relation & 90.7 & 9.6 \\
\hline 85 & 05406752 & Fancy Ck nr Bloom City & $43^{\circ} 27^{\prime} 26^{\prime \prime}$ & $90^{\circ} 29^{\prime} 58^{\prime \prime}$ & 05408000 & 4.14 & .3020 & equation & 2.1 & 6.8 \\
\hline 86 & 05406765 & Fancy Ck Tr nr Gillingham & $43^{\circ} 25^{\prime} 35^{\prime \prime}$ & $90^{\circ} 27^{\prime} 58^{\prime \prime}$ & 05408000 & 6.21 & .3763 & equation & 3.5 & 7.7 \\
\hline 87 & 05406770 & Fancy $\mathrm{Ck}$ Tr at Gillingham & $43^{\circ} 25^{\prime} 36^{\prime \prime}$ & $90^{\circ} 26^{\prime} 45^{\prime \prime}$ & 05408000 & 2.28 & .3682 & equation & 1.3 & 7.4 \\
\hline 88 & 05406780 & Fancy Ck nr Richland Center & $43^{\circ} 23^{\prime} 08^{\prime \prime}$ & $90^{\circ} 23^{\prime} 20^{\prime \prime}$ & 05408000 & 28.4 & -- & relation & 14.5 & 6.9 \\
\hline 89 & 05406781 & Horse Ck nr Gillingham & $43^{\circ} 22^{\prime} 37^{\prime \prime}$ & $90^{\circ} 26^{\prime} 44^{\prime \prime}$ & 05408000 & 3.44 & .4684 & equation & 2.2 & 8.5 \\
\hline 90 & 05406789 & Brush Ck at Richland Center & $43^{\circ} 20^{\prime} 42^{\prime \prime}$ & $90^{\circ} 23^{\prime} 41^{\prime \prime}$ & 05408000 & 6.77 & .3558 & equation & 3.7 & 7.5 \\
\hline 91 & 05406793 & Center $\mathrm{Ck}$ at Richland Center & $43^{\circ} 20^{\prime} 02^{\prime \prime}$ & $90^{\circ} 23^{\prime} 41^{\prime \prime}$ & 05408000 & 2.57 & .2882 & equation & 1.2 & 6.6 \\
\hline 92 & 05406795 & Pine R at Richland Center & $43^{\circ} 19^{\prime} 50^{\prime \prime}$ & $90^{\circ} 23^{\prime} 20^{\prime \prime}$ & 05408000 & 190 & -- & relation & 110.0 & 7.9 \\
\hline 93 & 05406820 & Pine R nr Richland Center & $43^{\circ} 18^{\prime} 29^{\prime \prime}$ & $90^{\circ} 20^{\prime} 58^{\prime \prime}$ & 05408000 & 198 & .5150 & relation & 129.0 & 8.8 \\
\hline 94 & 05406825 & Spring Ck nr Richland Center & $43^{\circ} 18^{\prime} 47^{\prime \prime}$ & $90^{\circ} 20^{\prime} 36^{\prime \prime}$ & 05408000 & 2.74 & .1648 & equation & 1.0 & 4.9 \\
\hline 95 & 05406845 & Ash Ck nr Richland Center & $43^{\circ} 16^{\prime} 28^{\prime \prime}$ & $90^{\circ} 22^{\prime} 42^{\prime \prime}$ & 05408000 & 7.95 & .3246 & equation & 4.2 & 7.2 \\
\hline 96 & 05406850 & Ash Ck nr Richland Center & $43^{\circ} 17^{\prime} 16^{\prime \prime}$ & $90^{\circ} 20^{\prime} 03^{\prime \prime}$ & 05408000 & 18.4 & .2510 & relation & 6.5 & 4.8 \\
\hline 97 & 05406851 & Pine $R$ at Twin Bluffs & $43^{\circ} 16^{\prime} 37^{\prime \prime}$ & $90^{\circ} 18^{\prime} 38^{\prime \prime}$ & 05408000 & 224 & .4694 & equation & 152.8 & 9.3 \\
\hline 98 & 05406853 & Willow Ck nr Loyd & $43^{\circ} 26^{\prime} 17^{\prime \prime}$ & $90^{\circ} 14^{\prime} 30^{\prime \prime}$ & 05408000 & 9.28 & .3159 & equation & 4.8 & 7.1 \\
\hline 99 & 05406858 & Willow Ck Tr nr Loyd & $43^{\circ} 25^{\prime} 25^{\prime \prime}$ & $90^{\circ} 13^{\prime} 00^{\prime \prime}$ & 05408000 & 3.67 & .1305 & equation & 1.2 & 4.4 \\
\hline 100 & 05406860 & Tr To Willow Ck Tr nr Loyd & $43^{\circ} 25^{\prime} 16^{\prime \prime}$ & $90^{\circ} 12^{\prime} 21^{\prime \prime}$ & 05408000 & 3.52 & .1061 & equation & 1.0 & 3.9 \\
\hline 101 & 05406863 & Willow Ck Tr nr Loyd & $43^{\circ} 24^{\prime} 41^{\prime \prime}$ & $90^{\circ} 14^{\prime} 12^{\prime \prime}$ & 05408000 & 9.96 & .3148 & equation & 5.2 & 7.1 \\
\hline 102 & 05406864 & Willow Ck Tr nr Loyd & $43^{\circ} 23^{\prime} 54^{\prime \prime}$ & $90^{\circ} 13^{\prime} 36^{\prime \prime}$ & 05408000 & 2.63 & .3141 & equation & 1.3 & 6.9 \\
\hline
\end{tabular}

${ }^{1}$ Low-flow discharge measurements at partial-record stations were compared to one or more nearby USGS gaging stations to establish a relation between measured discharge and concurrent daily discharge. The nearby gaging station that provided the best relation with the partial-record station was chosen as the index station and was associated with nearby miscellaneous measurement sites.

${ }^{2}$ Mismatch between surface-water and ground-water drainage area. 
[USGS, U.S. Geological Survey; ID, identification number; $\mathrm{dd}^{\circ} \mathrm{mm}$ 'ss", degrees, minutes, seconds; $\mathrm{mi}^{2}$, square mile; $\mathrm{ft}^{3} / \mathrm{s} / \mathrm{mi}^{2}$, cubic foot per second per square mile; $\mathrm{ft}^{3} / \mathrm{s}$, cubic foot per second; equation, statewide regression equation; relation, established relation line with index station; R, River; Bk, Brook; Br, Branch; Fk, Fork; Ck, Creek; Tr, Tributary; nr, near; N, North; S, South; E, East; W, West; ,-- not determined in historical low-flow reports. See references for full list of low-flow reports.]

\begin{tabular}{|c|c|c|c|c|c|c|c|c|c|c|}
\hline $\begin{array}{c}\text { Map } \\
\text { number }\end{array}$ & $\begin{array}{c}\text { USGS } \\
\text { station ID }\end{array}$ & Station name & $\begin{array}{c}\text { Latitude } \\
\text { (dd }{ }^{\circ} \mathrm{mm} \text { 'ss") }\end{array}$ & $\begin{array}{l}\text { Longitude } \\
\text { (ddºmm'ss") }\end{array}$ & $\begin{array}{l}\text { Index } \\
\text { station }\end{array}$ & $\begin{array}{c}\text { Drainage } \\
\text { area } \\
\left(\mathrm{mi}^{2}\right)\end{array}$ & $\begin{array}{c}\text { Base flow } \\
\text { factor } \\
\left(\mathrm{ft}^{3} / \mathrm{s} / \mathrm{mi}^{2}\right)\end{array}$ & $\begin{array}{l}\text { Base flow } \\
\text { method }\end{array}$ & $\begin{array}{c}\text { Base flow } \\
\qquad\left(\mathrm{ft}^{3} / \mathrm{s}\right)\end{array}$ & $\begin{array}{r}\text { Recharge } \\
\text { (inches) }\end{array}$ \\
\hline 103 & 05406865 & Willow Ck nr Richland Center & $43^{\circ} 23^{\prime} 41^{\prime \prime}$ & $90^{\circ} 14^{\prime} 30^{\prime \prime}$ & 05408000 & 32.4 & 0.5145 & equation & 22.3 & 9.3 \\
\hline 104 & 05406867 & Willow Ck Tr nr Loyd & $43^{\circ} 23^{\prime} 33^{\prime \prime}$ & $90^{\circ} 14^{\prime} 59^{\prime \prime}$ & 05408000 & 2.94 & .2290 & equation & 1.3 & 5.8 \\
\hline 105 & 05406875 & Willow Ck nr Richland Center & $43^{\circ} 19^{\prime} 54^{\prime \prime}$ & $90^{\circ} 17^{\prime} 27^{\prime \prime}$ & 05408000 & 54.7 & .5014 & equation & 37.5 & 9.3 \\
\hline 106 & 05406877 & Little Willow Ck nr Neptune & $43^{\circ} 22^{\prime} 35^{\prime \prime}$ & $90^{\circ} 18^{\prime} 24^{\prime \prime}$ & 05408000 & 6.71 & .3397 & equation & 3.6 & 7.3 \\
\hline 107 & 05406879 & Little Willow Ck Tr nr Ithaca & $43^{\circ} 21^{\prime} 05^{\prime \prime}$ & $90^{\circ} 18^{\prime} 19^{\prime \prime}$ & 05408000 & 2.32 & .3205 & equation & 1.2 & 6.9 \\
\hline 108 & 05406882 & $\begin{array}{l}\text { Little Willow Ck nr Richland } \\
\text { Center }\end{array}$ & $43^{\circ} 19^{\prime} 55^{\prime \prime}$ & $90^{\circ} 17^{\prime} 22^{\prime \prime}$ & 05408000 & 13.9 & .3332 & equation & 7.5 & 7.3 \\
\hline 109 & 05406885 & Willow Ck Tr nr Sextonville & $43^{\circ} 17^{\prime} 52^{\prime \prime}$ & $90^{\circ} 18^{\prime} 04^{\prime \prime}$ & 05408000 & 2.29 & .1360 & equation & .7 & 4.4 \\
\hline 110 & 05406886 & Willow Ck at Sextonville & $43^{\circ} 16^{\prime} 47^{\prime \prime}$ & $90^{\circ} 18^{\prime} 09^{\prime \prime}$ & 05408000 & 79.5 & .4597 & equation & 52.5 & 9.0 \\
\hline 111 & 05406889 & Willow $\mathrm{Ck} \mathrm{Tr}$ at Sextonville & $43^{\circ} 16^{\prime} 44^{\prime \prime}$ & $90^{\circ} 18^{\prime} 02^{\prime \prime}$ & 05408000 & 2.95 & .0725 & equation & .7 & 3.2 \\
\hline 112 & 05406890 & Willow Ck nr Richland Center & $43^{\circ} 16^{\prime} 44^{\prime \prime}$ & $90^{\circ} 18^{\prime} 12^{\prime \prime}$ & 05408000 & 82.5 & .5150 & relation & 47.7 & 7.9 \\
\hline 113 & 05407003 & Mill Ck nr Boaz & $43^{\circ} 23^{\prime} 38^{\prime \prime}$ & $90^{\circ} 33^{\prime} 14^{\prime \prime}$ & 05408000 & 19.5 & .3564 & equation & 11.0 & 7.6 \\
\hline 114 & 05407013 & Mill Ck nr Boaz & $43^{\circ} 17^{\prime} 57^{\prime \prime}$ & $90^{\circ} 31 ' 24^{\prime \prime}$ & 05408000 & 61.5 & .5220 & equation & 43.2 & 9.5 \\
\hline 115 & 054070182 & Foreman Hollow nr Montfort & $42^{\circ} 59^{\prime} 45^{\prime \prime}$ & $90^{\circ} 23^{\prime} 42^{\prime \prime}$ & 05406500 & 4.88 & .2676 & equation & 2.3 & 6.4 \\
\hline 116 & 054070187 & Blue R Tr nr Highland & $43^{\circ} 00^{\prime} 24^{\prime \prime}$ & $90^{\circ} 24^{\prime} 30^{\prime \prime}$ & 05406500 & 3.93 & .0088 & equation & .3 & 1.1 \\
\hline 117 & 05407021 & Blue R nr Highland & $43^{\circ} 01^{\prime} 53^{\prime \prime}$ & $90^{\circ} 27 ' 53^{\prime \prime}$ & 05406500 & 29.8 & .3902 & equation & 17.7 & 8.1 \\
\hline 118 & 054070215 & Big Rock Br nr Highland & $43^{\circ} 03^{\prime} 08^{\prime \prime}$ & $90^{\circ} 27^{\prime} 12^{\prime \prime}$ & 05406500 & 9.42 & .0575 & equation & 2.0 & 2.9 \\
\hline 119 & 05407023 & Blue R nr Muscoda & $43^{\circ} 05^{\prime} 37^{\prime \prime}$ & $90^{\circ} 29^{\prime} 03^{\prime \prime}$ & 05406500 & 50 & .3542 & equation & 28.6 & 7.8 \\
\hline 120 & 05407024 & Six Mile Br nr Muscoda & $43^{\circ} 06^{\prime} 18^{\prime \prime}$ & $90^{\circ} 28^{\prime} 09^{\prime \prime}$ & 05406500 & 11.3 & .2107 & equation & 4.8 & 5.8 \\
\hline 121 & 05407026 & Big Spring Br nr Muscoda & $43^{\circ} 04^{\prime} 40^{\prime \prime}$ & $90^{\circ} 25^{\prime} 03^{\prime \prime}$ & 05406500 & 7.26 & .1738 & equation & 2.8 & 5.2 \\
\hline 122 & 05407029 & Big Spring Br nr Muscoda & $43^{\circ} 06^{\prime} 15^{\prime \prime}$ & $90^{\circ} 29^{\prime} 30^{\prime \prime}$ & 05406500 & 12 & .3454 & equation & 6.6 & 7.4 \\
\hline 123 & 05407030 & Blue $\mathrm{R}$ nr Blue $\mathrm{R}$ & $43^{\circ} 07^{\prime} 41^{\prime \prime}$ & $90^{\circ} 30^{\prime} 44^{\prime \prime}$ & 05406500 & 79.6 & .4486 & equation & 51.9 & 8.9 \\
\hline 124 & 05407037 & Fennimore Fk nr Castle Rock & $43^{\circ} 00^{\prime} 33^{\prime \prime}$ & $90^{\circ} 34^{\prime} 56^{\prime \prime}$ & 05414000 & 7.1 & .1031 & equation & 2.1 & 3.9 \\
\hline 125 & 05407040 & Fennimore Fk nr Castle Rock & $43^{\circ} 02^{\prime} 12^{\prime \prime}$ & $90^{\circ} 33^{\prime} 31^{\prime \prime}$ & 05414000 & 23.3 & .0281 & equation & 3.5 & 2.0 \\
\hline 126 & 05407042 & Fennimore Fk Tr nr Castle Rock & $43^{\circ} 01^{\prime} 53^{\prime \prime}$ & $90^{\circ} 32^{\prime} 53^{\prime \prime}$ & 05414000 & 15.5 & .1734 & equation & 6.0 & 5.2 \\
\hline 127 & 05407044 & Fennimore Fk Tr at Castle Rock & $43^{\circ} 02^{\prime} 40^{\prime \prime}$ & $90^{\circ} 31^{\prime} 54^{\prime \prime}$ & 05414000 & 3.73 & .0186 & equation & .4 & 1.6 \\
\hline
\end{tabular}

${ }^{1}$ Low-flow discharge measurements at partial-record stations were compared to one or more nearby USGS gaging stations to establish a relation between measured discharge and concurrent daily discharge. The nearby gaging station that provided the best relation with the partial-record station was chosen as the index station and was associated with nearby miscellaneous measurement sites.

${ }^{2}$ Mismatch between surface-water and ground-water drainage area. 
Appendix 10D. Average annual base flow and corresponding recharge, 1970-99, for partial-record stations in the Lower Wisconsin River Basin-Continued.

[USGS, U.S. Geological Survey; ID, identification number; $\mathrm{dd}^{\circ} \mathrm{mm}$ 'ss", degrees, minutes, seconds; $\mathrm{mi}^{2}$, square mile; $\mathrm{ft}^{3} / \mathrm{s} / \mathrm{mi}^{2}$, cubic foot per second per square mile; $\mathrm{ft}^{3} / \mathrm{s}$, cubic foot per second; equation, statewide regression equation; relation, established relation line with index station; R, River; Bk, Brook; Br, Branch; Fk, Fork; Ck, Creek; Tr, Tributary; nr, near; N, North; S, South; E, East; W, West;

--, not determined in historical low-flow reports. See references for full list of low-flow reports.]

\begin{tabular}{|c|c|c|c|c|c|c|c|c|c|c|}
\hline $\begin{array}{c}\text { Map } \\
\text { number }\end{array}$ & $\begin{array}{c}\text { USGS } \\
\text { station ID }\end{array}$ & Station name & $\begin{array}{c}\text { Latitude } \\
\text { (dd }{ }^{\circ} \mathrm{mm} \text { 'ss") }\end{array}$ & $\begin{array}{l}\text { Longitude } \\
\text { (dd }{ }^{\circ} \mathrm{mm} \text { 'ss") }\end{array}$ & $\begin{array}{l}\text { Index } \\
\text { station }^{1}\end{array}$ & $\begin{array}{c}\text { Drainage } \\
\text { area } \\
\left(\mathrm{mi}^{2}\right)\end{array}$ & $\begin{array}{c}\text { Base flow } \\
\text { factor } \\
\left(\mathrm{ft}^{3} / \mathrm{s} / \mathrm{mi}^{2}\right)\end{array}$ & $\begin{array}{c}\text { Base flow } \\
\text { method }\end{array}$ & $\begin{array}{c}\text { Base flow } \\
\qquad\left(\mathrm{ft}^{3} / \mathrm{s}\right)\end{array}$ & $\begin{array}{c}\text { Recharge } \\
\text { (inches) }\end{array}$ \\
\hline 128 & 05407045 & Fennimore Fk Tr nr Blue R & $43^{\circ} 04^{\prime} 31^{\prime \prime}$ & $90^{\circ} 33^{\prime} 17^{\prime \prime}$ & 05414000 & 10.7 & 0.1989 & equation & 4.4 & 5.6 \\
\hline 129 & 05407050 & Fennimore Fk nr Blue R & $43^{\circ} 07^{\prime} 06^{\prime \prime}$ & $90^{\circ} 32^{\prime} 00^{\prime \prime}$ & 05414000 & 77.2 & .4180 & relation & 34.0 & 6.0 \\
\hline 130 & 05407054 & Blue R nr Boscobel & $43^{\circ} 08^{\prime} 12^{\prime \prime}$ & $90^{\circ} 31^{\prime} 13^{\prime \prime}$ & 05406500 & 161 & .4081 & equation & 101.4 & 8.6 \\
\hline 131 & 05407061 & Sand Br nr Muscoda & $43^{\circ} 09^{\prime} 19^{\prime \prime}$ & $90^{\circ} 29^{\prime} 29^{\prime \prime}$ & 05406500 & 8.42 & .2787 & equation & 4.1 & 6.6 \\
\hline 132 & 05407063 & Blue R nr Blue R & $43^{\circ} 10^{\prime} 30^{\prime \prime}$ & $90^{\circ} 30^{\prime} 21^{\prime \prime}$ & 05406500 & 186 & -- & relation & 87.0 & 6.4 \\
\hline 133 & 05407064 & Blue $\mathrm{R}$ at Blue $\mathrm{R}$ & $43^{\circ} 11^{\prime} 20^{\prime \prime}$ & $90^{\circ} 33^{\prime} 40^{\prime \prime}$ & 05406500 & 193 & .3515 & equation & 112.9 & 7.9 \\
\hline 134 & 05407078 & W Fk Knapp Ck nr Excelsior & $43^{\circ} 15^{\prime} 47^{\prime \prime}$ & $90^{\circ} 39^{\prime} 17^{\prime \prime}$ & 05410500 & 17.1 & .4970 & equation & 11.4 & 9.1 \\
\hline 135 & 05407080 & Knapp Ck nr Wport & $43^{\circ} 12^{\prime} 19^{\prime \prime}$ & $90^{\circ} 38^{\prime} 25^{\prime \prime}$ & 05410500 & 85.1 & .5290 & relation & 46.0 & 7.3 \\
\hline 136 & 05407100 & Richland Ck nr Plugtown & $43^{\circ} 11^{\prime} 12^{\prime \prime}$ & $90^{\circ} 44^{\prime} 24^{\prime \prime}$ & 05410500 & 19.2 & .5880 & relation & 11.1 & 7.9 \\
\hline 137 & 05407130 & Saunders Ck at Boscobel & $43^{\circ} 08^{\prime} 39^{\prime \prime}$ & $90^{\circ} 42^{\prime} 27^{\prime \prime}$ & 05413500 & 17.1 & -- & relation & 6.5 & 5.2 \\
\hline 138 & 05407200 & Crooked Ck nr Boscobel & $43^{\circ} 06^{\prime} 27^{\prime \prime}$ & $90^{\circ} 42^{\prime} 19^{\prime \prime}$ & 05413500 & 12.9 & .6680 & relation & 8.8 & 9.3 \\
\hline 139 & 05407250 & Big Green R nr Wauzeka & $43^{\circ} 01^{\prime} 16^{\prime \prime}$ & $90^{\circ} 46^{\prime} 34^{\prime \prime}$ & 05413500 & 22.5 & .2489 & equation & 10.5 & 6.4 \\
\hline 140 & 05407260 & Big Green R nr Wauzeka & $43^{\circ} 03^{\prime} 54^{\prime \prime}$ & $90^{\circ} 50^{\prime} 18^{\prime \prime}$ & 05413500 & 48.4 & .3916 & equation & 29.1 & 8.2 \\
\hline 141 & 05407280 & Little Green R nr Wauzeka & $43^{\circ} 03^{\prime} 22^{\prime \prime}$ & $90^{\circ} 50^{\prime} 29^{\prime \prime}$ & 05413500 & 16.5 & .3730 & equation & 9.5 & 7.8 \\
\hline 142 & 05407360 & Kickapoo R at Wilton & $43^{\circ} 48^{\prime} 40^{\prime \prime}$ & $90^{\circ} 31^{\prime} 56^{\prime \prime}$ & 05408000 & 34.9 & -- & relation & 17.8 & 6.9 \\
\hline 143 & 05407380 & Pee Ck nr Wilton & $43^{\circ} 47^{\prime} 21^{\prime \prime}$ & $90^{\circ} 32^{\prime} 27^{\prime \prime}$ & 05408000 & 9.87 & .2180 & equation & 4.2 & 5.8 \\
\hline 144 & 05407400 & Morris Ck Tr nr Norwalk & $43^{\circ} 51^{\prime} 10^{\prime \prime}$ & $90^{\circ} 37^{\prime} 32^{\prime \prime}$ & 05383000 & 4.59 & .2150 & relation & 1.8 & 5.3 \\
\hline 145 & 05407411 & Morris $\operatorname{Tr}$ at Norwalk & $43^{\circ} 49^{\prime} 41^{\prime \prime}$ & $90^{\circ} 36^{\prime} 55^{\prime \prime}$ & 05408000 & 19.9 & -- & relation & 8.4 & 5.7 \\
\hline 146 & 05407470 & Kickapoo R at Ontario & $43^{\circ} 43^{\prime} 18^{\prime \prime}$ & $90^{\circ} 35^{\prime} 15^{\prime \prime}$ & 05408000 & 117 & -- & relation & 58.0 & 6.7 \\
\hline 147 & 05407490 & Brush $\mathrm{Ck}$ at Ontario & $43^{\circ} 43^{\prime} 32^{\prime \prime}$ & $90^{\circ} 36^{\prime} 19^{\prime \prime}$ & 05408000 & 31.2 & .3957 & equation & 18.7 & 8.1 \\
\hline 148 & 05407500 & Kickapoo R at Ontario & $43^{\circ} 42^{\prime} 52^{\prime \prime}$ & $90^{\circ} 35^{\prime} 13^{\prime \prime}$ & 05408000 & 151 & -- & relation & 84.0 & 7.6 \\
\hline 149 & 05407710 & Billings Ck nr Ontario & $43^{\circ} 41^{\prime} 16^{\prime \prime}$ & $90^{\circ} 33^{\prime} 56^{\prime \prime}$ & 05408000 & 23 & -- & relation & 16.8 & 9.9 \\
\hline 150 & 05407800 & Warner Ck nr La Farge & $43^{\circ} 39^{\prime} 06^{\prime \prime}$ & $90^{\circ} 35^{\prime} 40^{\prime \prime}$ & 05408000 & 24.9 & .5100 & equation & 17.0 & 9.3 \\
\hline 151 & 05407900 & Weistee Ck nr La Farge & $43^{\circ} 37^{\prime} 55^{\prime \prime}$ & $90^{\circ} 38^{\prime} 52^{\prime \prime}$ & 05408000 & 18 & .4540 & equation & 11.5 & 8.7 \\
\hline 152 & 05408200 & Bear Ck nr La Farge & $43^{\circ} 34^{\prime} 27^{\prime \prime}$ & $90^{\circ} 37^{\prime} 24^{\prime \prime}$ & 05408000 & 23.4 & .3980 & equation & 14.0 & 8.1 \\
\hline 153 & 05408300 & Camp Ck nr Viola & $43^{\circ} 30^{\prime} 07^{\prime \prime}$ & $90^{\circ} 38^{\prime} 59^{\prime \prime}$ & 05408000 & 16 & .6526 & equation & 12.3 & 10.4 \\
\hline 154 & 05408700 & W Fk Kickapoo R nr Viroqua & $43^{\circ} 33^{\prime} 44^{\prime \prime}$ & $90^{\circ} 45^{\prime} 45^{\prime \prime}$ & 05408500 & 63.5 & .4490 & relation & 33.0 & 7.1 \\
\hline
\end{tabular}

${ }^{1}$ Low-flow discharge measurements at partial-record stations were compared to one or more nearby USGS gaging stations to establish a relation between measured discharge and concurrent daily discharge. The nearby gaging station that provided the best relation with the partial-record station was chosen as the index station and was associated with nearby miscellaneous measurement sites.

${ }^{2}$ Mismatch between surface-water and ground-water drainage area. 
Appendix 10D. Average annual base flow and corresponding recharge, 1970-99, for partial-record stations in the Lower Wisconsin River Basin—Continued.

[USGS, U.S. Geological Survey; ID, identification number; $\mathrm{dd}^{\circ} \mathrm{mm}$ 'ss", degrees, minutes, seconds; $\mathrm{mi}^{2}$, square mile; $\mathrm{ft}^{3} / \mathrm{s} / \mathrm{mi}^{2}$, cubic foot per second per square mile; $\mathrm{ft}^{3} / \mathrm{s}$, cubic foot per second; equation, statewide regression equation; relation, established relation line with index station; R, River; Bk, Brook; Br, Branch; Fk, Fork; Ck, Creek; Tr, Tributary; nr, near; N, North; S, South; E, East; W, West; ,-- not determined in historical low-flow reports. See references for full list of low-flow reports.]

\begin{tabular}{|c|c|c|c|c|c|c|c|c|c|c|}
\hline $\begin{array}{c}\text { Map } \\
\text { number }\end{array}$ & $\begin{array}{c}\text { USGS } \\
\text { station ID }\end{array}$ & Station name & $\begin{array}{c}\text { Latitude } \\
\text { (dd }{ }^{\circ} \mathrm{mm} \text { 'ss") }\end{array}$ & $\begin{array}{l}\text { Longitude } \\
\text { (dd }{ }^{\circ} \mathrm{mm} \text { 'ss") }\end{array}$ & $\begin{array}{l}\text { Index } \\
\text { station }^{1}\end{array}$ & $\begin{array}{c}\text { Drainage } \\
\text { area } \\
\left(\mathrm{mi}^{2}\right)\end{array}$ & $\begin{array}{c}\text { Base flow } \\
\text { factor } \\
\left(\mathrm{ft}^{3} / \mathrm{s} / \mathrm{mi}^{2}\right)\end{array}$ & $\begin{array}{c}\text { Base flow } \\
\text { method }\end{array}$ & $\begin{array}{c}\text { Base flow } \\
\quad\left(\mathrm{ft}^{3} / \mathrm{s}\right)\end{array}$ & $\begin{array}{r}\text { Recharge } \\
\text { (inches) }\end{array}$ \\
\hline 155 & 05408800 & Bishops Ck nr Viroqua & $43^{\circ} 32^{\prime} 55^{\prime \prime}$ & $90^{\circ} 49^{\prime} 15^{\prime \prime}$ & 05408000 & 7.27 & 0.4090 & relation & 4.0 & 7.5 \\
\hline 156 & 05409000 & W Fk Kickapoo R nr Readstown & $43^{\circ} 28^{\prime} 35^{\prime \prime}$ & $90^{\circ} 44^{\prime} 30^{\prime \prime}$ & 05408000 & 106 & -- & relation & 53.0 & 6.8 \\
\hline 157 & 05409500 & Kickapoo R at Soldiers Grove & $43^{\circ} 23^{\prime} 40^{\prime \prime}$ & $90^{\circ} 46^{\prime} 35^{\prime \prime}$ & 05408000 & 530 & -- & relation & 284.0 & 7.3 \\
\hline 158 & 05409800 & Tainter Ck nr Gays Mills & $43^{\circ} 22^{\prime} 13^{\prime \prime}$ & $90^{\circ} 51^{\prime} 41^{\prime \prime}$ & 05408000 & 38.3 & .5150 & relation & 27.6 & 9.8 \\
\hline 159 & 05409860 & S Fk Nederlo Ck nr Gays Mills & $43^{\circ} 21^{\prime} 36^{\prime \prime}$ & $90^{\circ} 54^{\prime} 31^{\prime \prime}$ & 05409890 & 4.11 & -- & relation & 1.2 & 4.0 \\
\hline 160 & 05409870 & Nederlo Ck nr Gays Mills & $43^{\circ} 21^{\prime} 30^{\prime \prime}$ & $90^{\circ} 53^{\prime} 49^{\prime \prime}$ & 05409890 & 6.7 & -- & relation & 2.4 & 4.9 \\
\hline 161 & 05410600 & Pine Ck nr Steuben & $43^{\circ} 10^{\prime} 25^{\prime \prime}$ & $90^{\circ} 53^{\prime} 39^{\prime \prime}$ & 05413500 & 26.6 & .4860 & relation & 9.8 & 5.0 \\
\hline 162 & 05410650 & Plum Ck nr Wauzeka & $43^{\circ} 07^{\prime} 41^{\prime \prime}$ & $90^{\circ} 54^{\prime} 42^{\prime \prime}$ & 05413500 & 16.4 & .3656 & equation & 9.3 & 7.7 \\
\hline 163 & 05410750 & Little Kickapoo Ck nr Wauzeka & $43^{\circ} 04^{\prime} 41^{\prime \prime}$ & $90^{\circ} 55^{\prime} 51^{\prime \prime}$ & 05413500 & 9.94 & .1378 & equation & 3.4 & 4.6 \\
\hline 164 & 05410850 & Millville Ck nr Wauzeka & $43^{\circ} 01^{\prime} 55^{\prime \prime}$ & $90^{\circ} 55^{\prime} 50^{\prime \prime}$ & 05413500 & 16.3 & .3845 & equation & 9.5 & 7.9 \\
\hline 165 & 05410910 & Dutch Hollow Ck nr Wauzeka & $43^{\circ} 02^{\prime} 02^{\prime \prime}$ & $90^{\circ} 57^{\prime} 34^{\prime \prime}$ & 05413500 & 4.57 & .0780 & equation & 1.1 & 3.4 \\
\hline 166 & 05411000 & Gran Grae Ck nr Wauzeka & $43^{\circ} 02^{\prime} 36^{\prime \prime}$ & $90^{\circ} 59^{\prime} 15^{\prime \prime}$ & 05413500 & 17.1 & .3800 & relation & 6.3 & 5.0 \\
\hline
\end{tabular}

${ }^{1}$ Low-flow discharge measurements at partial-record stations were compared to one or more nearby USGS gaging stations to establish a relation between measured discharge and concurrent daily discharge. The nearby gaging station that provided the best relation with the partial-record station was chosen as the index station and was associated with nearby miscellaneous measurement sites

${ }^{2}$ Mismatch between surface-water and ground-water drainage area. 

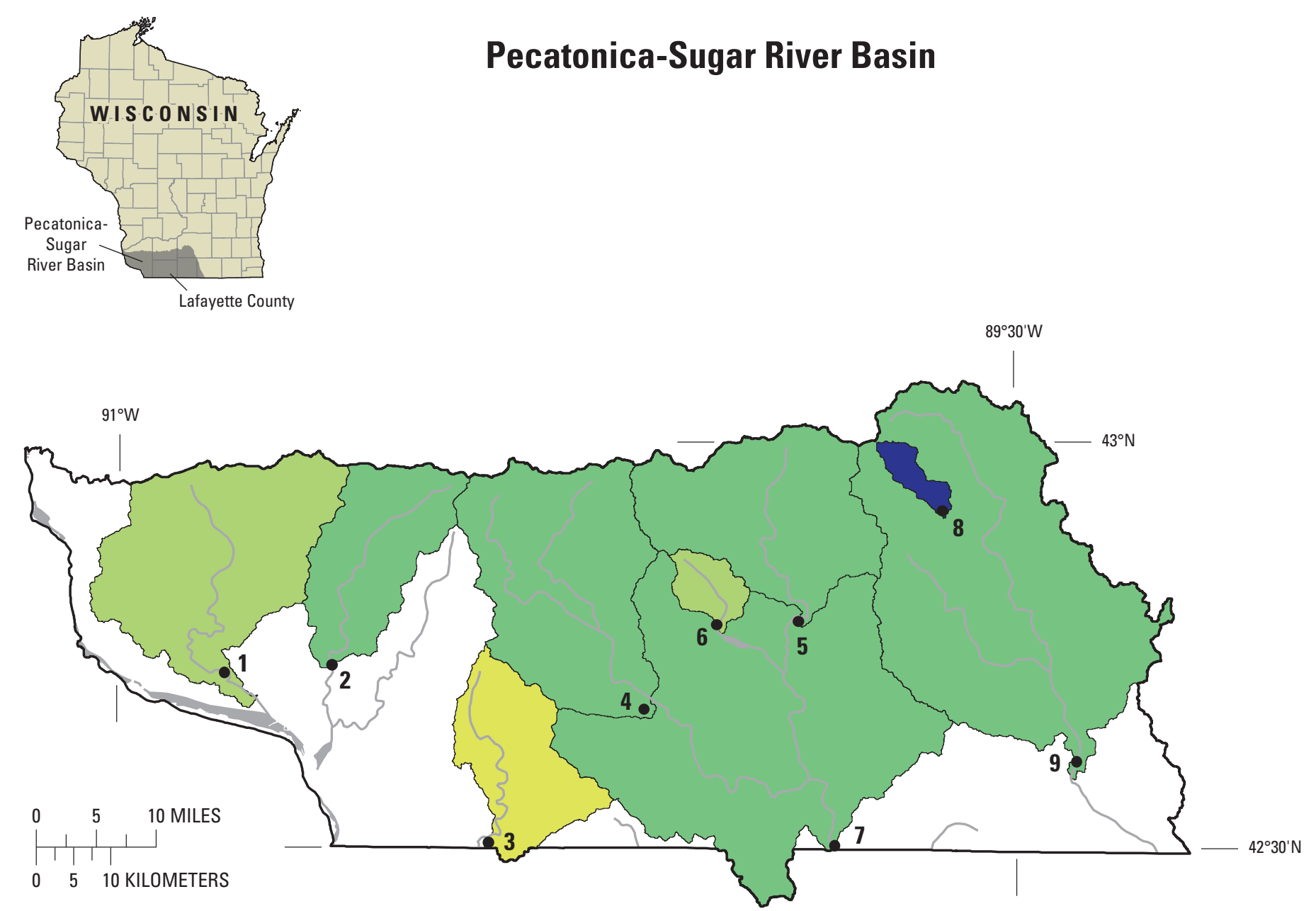

Appendix 11-A. Locations of streamflow-gaging stations in the Pecatonica-Sugar River Basin and spatial distribution of average annual recharge.

\begin{tabular}{|c|c|}
\hline & EXPLANATION \\
\hline \multicolumn{2}{|c|}{ Recharge, in inches } \\
\hline & $\geq 12$ \\
\hline & $11-11.9$ \\
\hline & $10-10.9$ \\
\hline & $9-9.9$ \\
\hline & $8-8.9$ \\
\hline & $7-7.9$ \\
\hline & $6-6.9$ \\
\hline & $5-5.9$ \\
\hline & $4-4.9$ \\
\hline & $3-3.9$ \\
\hline & $2-2.9$ \\
\hline & $1-1.9$ \\
\hline & $<1$ \\
\hline & Open water \\
\hline & Basin boundary \\
\hline & Subbasin boundary \\
\hline & Stream \\
\hline${ }^{4} \bullet$ & $\begin{array}{l}\text { U.S. Geological Survey } \\
\text { streamflow-gaging station }\end{array}$ \\
\hline
\end{tabular}

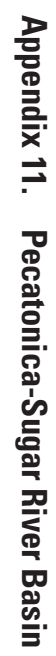




\section{Pecatonica-Sugar River Basin}

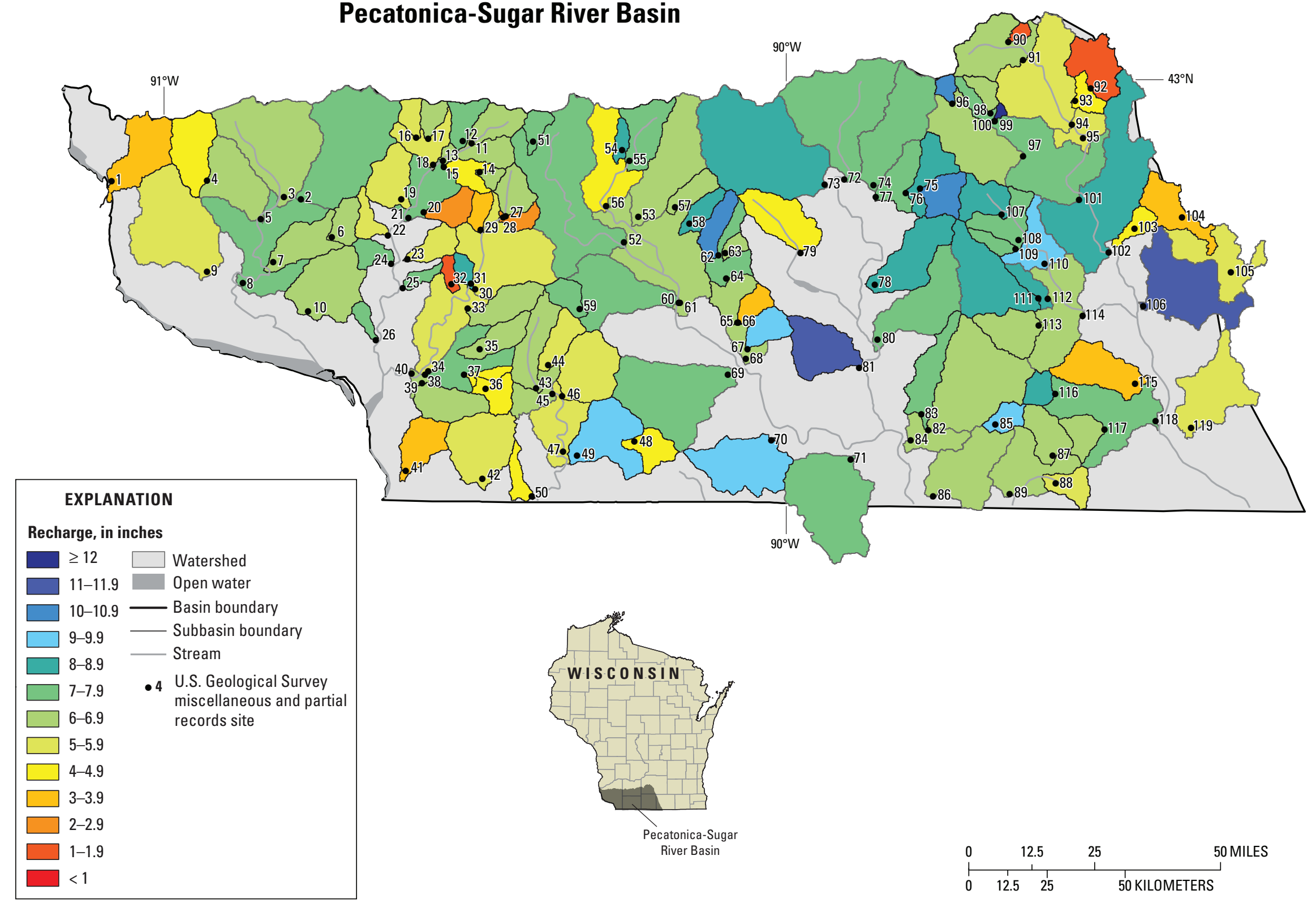

Appendix 11-B. Locations of miscellaneous and partial-record sites in the Pecatonica-Sugar River Basin and spatial distribution of estimated average annual recharge. 
Appendix 11C. Average annual base flow and corresponding recharge, 1970-99, for streamflow-gaging stations in the Pecatonica-Sugar River Basin.

[USGS, U.S. Geological Survey; ID, identification number; mi² $^{2}$ square mile; ft³/s, cubic foot per second; BFI, Baseflow Index; ex rec, extended record; R, River; Bk, Brook; Br, Branch; Fk, Fork; Ck, Creek; Tr, Tributary; nr, near; N, North; S, South; E, East; W, West; --, not determined in historical low-flow reports. See references for full list of low-flow reports.]

\begin{tabular}{|c|c|c|c|c|c|c|c|c|c|c|c|}
\hline $\begin{array}{c}\text { Map } \\
\text { number }\end{array}$ & $\begin{array}{c}\text { USGS } \\
\text { station } \\
\text { ID }\end{array}$ & Station name & $\begin{array}{c}\text { Drainage } \\
\text { area } \\
\left(\mathrm{mi}^{2}\right)\end{array}$ & $\begin{array}{c}\text { Period of } \\
\text { analysis } \\
\text { (water years }{ }^{1} \text { ) }\end{array}$ & $\begin{array}{c}\text { Base flow } \\
\text { method }\end{array}$ & $\begin{array}{l}\text { Base flow } \\
\text { index }\end{array}$ & $\begin{array}{c}\text { Base flow } \\
\quad\left(\mathrm{ft}^{3} / \mathrm{s}\right)\end{array}$ & $\begin{array}{c}\text { Recharge } \\
\text { (inches) }\end{array}$ & $\begin{array}{c}\text { Total } \\
\text { streamflow } \\
\left(\mathrm{ft}^{3} / \mathbf{s}\right)\end{array}$ & $\begin{array}{c}\text { Total } \\
\text { streamflow } \\
\text { (inches) }\end{array}$ & $\begin{array}{l}2090 \\
\left(\mathrm{ftt}^{3} / \mathrm{s}\right)\end{array}$ \\
\hline 1 & 05413500 & Grant $\mathrm{R}$ at Burton & 269 & 1970-1999 & BFI & 0.7560 & 140 & 6.8 & 180 & 9.1 & 84 \\
\hline 2 & 05414000 & Platte R nr Rockville & 142 & 1970-1999 & BFI & .7450 & 78 & 7.5 & 110 & 10.1 & 44 \\
\hline 3 & 05415000 & Galena $\mathrm{R}$ at Buncombe & 125 & 1948-1992 & ex rec & -- & 52 & 5.6 & -- & -- & 28 \\
\hline 4 & 05432500 & Pecatonica $\mathrm{R}$ at Darlington & 273 & 1970-1999 & BFI & .6900 & 140 & 7.1 & 210 & 10.4 & 75 \\
\hline 5 & 05433000 & E Br Pecatonica R nr Blanchardville & 221 & 1970-1999 & BFI & .7760 & 130 & 8.0 & 170 & 10.4 & 78 \\
\hline 6 & 05433500 & Yellowstone R nr Blanchardville & 28.5 & $1955-1979$ & ex rec & -- & 14 & 6.7 & -- & -- & 8.0 \\
\hline 7 & 05434500 & Pecatonica $\mathrm{R}$ at Martintown & 1034 & 1970-1999 & BFI & .7510 & 600 & 7.9 & 810 & 10.6 & 330 \\
\hline 8 & 05436000 & Mt Vernon Ck nr Mt Vernon & 16.4 & $1955-1980$ & ex rec & -- & 18 & 14.9 & -- & -- & 14 \\
\hline 9 & 05436500 & Sugar R nr Brodhead & 523 & 1970-1999 & BFI & .7490 & 290 & 7.6 & 400 & 10.3 & 180 \\
\hline
\end{tabular}

${ }^{1}$ Water year is a 12-month period from October 1 through September 30, and is designated by the calendar year in which it ends (for example, the 2004 water year occurred October 1, 2003 through September 30, 2004).

${ }^{2}$ Discharge that is exceeded 90 percent of the time. 
[USGS, U.S. Geological Survey; ID, identification number; $\mathrm{dd}^{\circ} \mathrm{mm}$ 'ss", degrees, minutes, seconds; $\mathrm{mi}^{2}$, square mile; $\mathrm{ft}^{3} / \mathrm{s} / \mathrm{mi}^{2}$, cubic foot per second per square mile; $\mathrm{ft}^{3} / \mathrm{s}$, cubic foot per second; equation, statewide regression equation; relation, established relation line with index station; R, River; Bk, Brook; Br, Branch; Fk, Fork; Ck, Creek; Tr, Tributary; nr, near; N, North; S, South; E, East; W, West;

,-- not determined in historical low-flow reports. See references for full list of low-flow reports.]

\begin{tabular}{|c|c|c|c|c|c|c|c|c|c|c|}
\hline $\begin{array}{c}\text { Map } \\
\text { number }\end{array}$ & $\begin{array}{c}\text { USGS } \\
\text { station ID }\end{array}$ & Station name & $\begin{array}{c}\text { Latitude } \\
\text { (dd }{ }^{\circ} \mathrm{mm} \text { 'ss") }\end{array}$ & $\begin{array}{l}\text { Longitude } \\
\text { (ddºmm'ss") }\end{array}$ & $\begin{array}{l}\text { Index } \\
\text { station' }\end{array}$ & $\begin{array}{c}\text { Drainage } \\
\text { area } \\
\left(\mathrm{mi}^{2}\right)\end{array}$ & $\begin{array}{l}\text { Base flow } \\
\text { factor } \\
\left(\mathrm{ft}^{3} / \mathrm{s} / \mathrm{mi}^{2}\right)\end{array}$ & $\begin{array}{l}\text { Base flow } \\
\text { method }\end{array}$ & $\begin{array}{c}\text { Base flow } \\
\left(\mathrm{ft}^{3} / \mathrm{s}\right)\end{array}$ & $\begin{array}{c}\text { Recharge } \\
\text { (inches) }\end{array}$ \\
\hline 1 & 05411510 & Sandy Ck nr Bagley & $42^{\circ} 52^{\prime} 45^{\prime \prime}$ & $91^{\circ} 04^{\prime} 30^{\prime \prime}$ & 05413500 & 18.9 & 0.0290 & relation & 5.3 & 3.8 \\
\hline 2 & 05413000 & Grant R nr Lancaster & $42^{\circ} 51^{\prime} 37^{\prime \prime}$ & $90^{\circ} 46^{\prime} 22^{\prime \prime}$ & 05413500 & 50.9 & .3407 & equation & 28.5 & 7.6 \\
\hline 3 & 05413100 & Little Grant R nr Lancaster & $42^{\circ} 51^{\prime} 47^{\prime \prime}$ & $90^{\circ} 47^{\prime} 59^{\prime \prime}$ & 05413500 & 45.5 & .3540 & relation & 21.8 & 6.5 \\
\hline 4 & 05413140 & Blake Fk at Bloomington & $42^{\circ} 52^{\prime} 51^{\prime \prime}$ & $90^{\circ} 55^{\prime} 22^{\prime \prime}$ & 05413500 & 17.9 & .2260 & relation & 6.5 & 4.9 \\
\hline 5 & 05413250 & Blake Fk nr Beetown & $42^{\circ} 50^{\prime} 11^{\prime \prime}$ & $90^{\circ} 50^{\prime} 11^{\prime \prime}$ & 05413500 & 39.7 & .1900 & equation & 16.3 & 5.6 \\
\hline 6 & 05413400 & Pigeon Ck nr Lancaster & $42^{\circ} 48^{\prime} 59^{\prime \prime}$ & $90^{\circ} 43 ' 24^{\prime \prime}$ & 05413500 & 6.93 & .4390 & relation & 3.2 & 6.3 \\
\hline 7 & 05413415 & Pigeon Ck nr Beetown & $42^{\circ} 47^{\prime} 11^{\prime \prime}$ & $90^{\circ} 48^{\prime} 59^{\prime \prime}$ & 05413500 & 21 & .2788 & equation & 10.4 & 6.7 \\
\hline 8 & 05413430 & Grant R nr Beetown & $42^{\circ} 45^{\prime} 43^{\prime \prime}$ & $90^{\circ} 51^{\prime} 51^{\prime \prime}$ & 05413500 & 195 & .2911 & equation & 103.5 & 7.2 \\
\hline 9 & 05413450 & Rattlesnake Ck nr Cassville & $42^{\circ} 46^{\prime} 28^{\prime \prime}$ & $90^{\circ} 55^{\prime} 18^{\prime \prime}$ & 05413500 & 44.7 & .2060 & relation & 17.4 & 5.3 \\
\hline 10 & 05413600 & Boice Ck nr Potosi & $42^{\circ} 43^{\prime} 43^{\prime \prime}$ & $90^{\circ} 45^{\prime} 35^{\prime \prime}$ & 05413500 & 26.3 & .3480 & relation & 12.9 & 6.7 \\
\hline 11 & 05413700 & Platte R nr Livingston & $42^{\circ} 55^{\prime} 38^{\prime \prime}$ & $90^{\circ} 30^{\prime} 06^{\prime \prime}$ & 05414000 & 9.67 & .3035 & equation & 4.9 & 6.9 \\
\hline 12 & 05413720 & Platte R Tr nr Livingston & $42^{\circ} 55^{\prime} 49^{\prime \prime}$ & $90^{\circ} 30^{\prime} 57^{\prime \prime}$ & 05414000 & 6.78 & .3201 & equation & 3.5 & 7.1 \\
\hline 13 & 05413750 & Plate R nr Stitzer & $42^{\circ} 54^{\prime} 23^{\prime \prime}$ & $90^{\circ} 32^{\prime} 48^{\prime \prime}$ & 05414000 & 27 & .2963 & equation & 13.9 & 7.0 \\
\hline 14 & 05413770 & Crow $\mathrm{Br}$ nr Livingston & $42^{\circ} 53^{\prime} 37^{\prime \prime}$ & $90^{\circ} 29^{\prime} 18^{\prime \prime}$ & 05414000 & 2.17 & .2920 & equation & 1.0 & 6.3 \\
\hline 15 & 05413780 & Crow Br nr Stitzer & $42^{\circ} 53^{\prime} 56^{\prime \prime}$ & $90^{\circ} 32^{\prime} 45^{\prime \prime}$ & 05414000 & 8.89 & .2420 & relation & 3.0 & 4.6 \\
\hline 16 & 05413790 & Leggett Ck nr Stitzer & $42^{\circ} 56^{\prime} 00^{\prime \prime}$ & $90^{\circ} 35^{\prime} 25^{\prime \prime}$ & 05414000 & 4.45 & .2051 & equation & 1.8 & 5.6 \\
\hline 17 & 05413805 & Newell Ck nr Stitzer & $42^{\circ} 55^{\prime} 57^{\prime \prime}$ & $90^{\circ} 34^{\prime} 14^{\prime \prime}$ & 05414000 & 4.64 & .2518 & equation & 2.1 & 6.2 \\
\hline 18 & 05413810 & Leggett Ck nr Stitzer & $42^{\circ} 54^{\prime} 08^{\prime \prime}$ & $90^{\circ} 33^{\prime} 45^{\prime \prime}$ & 05414000 & 18.4 & .2009 & equation & 7.6 & 5.6 \\
\hline 19 & 05413850 & Platte R Tr \#2 nr Lancaster & $42^{\circ} 51^{\prime} 42^{\prime \prime}$ & $90^{\circ} 36^{\prime} 46^{\prime \prime}$ & 05414000 & 13.3 & .1726 & equation & 5.1 & 5.2 \\
\hline 20 & 05413880 & Bacon Br nr Ellenboro & $42^{\circ} 50^{\prime} 46^{\prime \prime}$ & $90^{\circ} 34^{\prime} 38^{\prime \prime}$ & 05414000 & 7.56 & .0850 & relation & 1.6 & 2.9 \\
\hline 21 & 05413900 & Platte R nr Lancaster & $42^{\circ} 50^{\prime} 22^{\prime \prime}$ & $90^{\circ} 36^{\prime} 06^{\prime \prime}$ & 05414000 & 90.8 & .2990 & relation & 39.8 & 6.0 \\
\hline 22 & 05413930 & Austin Br nr Ellenboro & $42^{\circ} 49^{\prime} 07^{\prime \prime}$ & $90^{\circ} 38^{\prime} 03^{\prime \prime}$ & 05414000 & 5.52 & .4810 & relation & 4.1 & 10.1 \\
\hline 23 & 05413960 & Willow Br nr Ellenboro & $42^{\circ} 47^{\prime} 27^{\prime \prime}$ & $90^{\circ} 36^{\prime} 08^{\prime \prime}$ & 05414000 & 7.88 & .1846 & equation & 3.1 & 5.3 \\
\hline 24 & 05413970 & Mcpherson Br nr Ellenboro & $42^{\circ} 47^{\prime} 09^{\prime \prime}$ & $90^{\circ} 37^{\prime} 43^{\prime \prime}$ & 05414000 & 5.12 & .4147 & equation & 3.0 & 8.0 \\
\hline 25 & 05413980 & Culver Br nr Ellenboro & $42^{\circ} 45^{\prime} 26^{\prime \prime}$ & $90^{\circ} 36^{\prime} 35^{\prime \prime}$ & 05414000 & 3.11 & .3377 & equation & 1.6 & 7.2 \\
\hline
\end{tabular}

${ }^{1}$ Low-flow discharge measurements at partial-record stations were compared to one or more nearby USGS gaging stations to establish a relation between measured discharge and concurrent daily discharge.

The nearby gaging station that provided the best relation with the partial-record station was chosen as the index station and was associated with nearby miscellaneous measurement sites.

${ }^{2}$ Mismatch between surface-water and ground-water drainage area. 
Appendix 11D. Average annual base flow and corresponding recharge, 1970-99, for partial-record stations in the Pecatonica-Sugar River Basin-Continued.

[USGS, U.S. Geological Survey; ID, identification number; $\mathrm{dd}^{\circ} \mathrm{mm}$ 'ss", degrees, minutes, seconds; $\mathrm{mi}^{2}$, square mile; $\mathrm{ft}^{3} / \mathrm{s} / \mathrm{mi}^{2}$, cubic foot per second per square mile; $\mathrm{ft}^{3} / \mathrm{s}$, cubic foot per second; equation, statewide regression equation; relation, established relation line with index station; R, River; Bk, Brook; Br, Branch; Fk, Fork; Ck, Creek; Tr, Tributary; nr, near; N, North; S, South; E, East; W, West; ,-- not determined in historical low-flow reports. See references for full list of low-flow reports.]

\begin{tabular}{|c|c|c|c|c|c|c|c|c|c|c|}
\hline $\begin{array}{c}\text { Map } \\
\text { number }\end{array}$ & $\begin{array}{c}\text { USGS } \\
\text { station ID }\end{array}$ & Station name & $\begin{array}{c}\text { Latitude } \\
\text { (dd }{ }^{\circ} \mathrm{mm} \text { 'ss") }\end{array}$ & $\begin{array}{c}\text { Longitude } \\
\text { (ddºmm'ss") }\end{array}$ & $\begin{array}{l}\text { Index } \\
\text { station }^{1}\end{array}$ & $\begin{array}{c}\text { Drainage } \\
\text { area } \\
\left(\mathrm{mi}^{2}\right)\end{array}$ & $\begin{array}{l}\text { Base flow } \\
\text { factor } \\
\left(\mathrm{ft}^{3} / \mathrm{s} / \mathrm{mi}^{2}\right)\end{array}$ & $\begin{array}{l}\text { Base flow } \\
\text { method }\end{array}$ & $\begin{array}{c}\text { Base flow } \\
\left(\mathrm{ft}^{3} / \mathrm{s}\right)\end{array}$ & $\begin{array}{c}\text { Recharge } \\
\text { (inches) }\end{array}$ \\
\hline 26 & 05414030 & Platte R Tr \#3 nr Tennyson & $42^{\circ} 41^{\prime} 46^{\prime \prime}$ & $90^{\circ} 39^{\prime} 08^{\prime \prime}$ & 05414000 & 7.19 & 0.3353 & equation & 3.8 & 7.2 \\
\hline 27 & 05414060 & Little Platte R at Arthur & $42^{\circ} 50^{\prime} 33^{\prime \prime}$ & $90^{\circ} 26^{\prime} 49^{\prime \prime}$ & 05414000 & 9 & .2743 & equation & 4.3 & 6.6 \\
\hline 28 & 05414070 & Little Platte R Tr at Arthur & $42^{\circ} 50^{\prime} 30^{\prime \prime}$ & $90^{\circ} 26^{\prime} 51^{\prime \prime}$ & 05414000 & 3.48 & .0564 & equation & .7 & 2.8 \\
\hline 29 & 05414080 & Little Platte R Tr nr Arthur & $42^{\circ} 49^{\prime} 33^{\prime \prime}$ & $90^{\circ} 29^{\prime} 11^{\prime \prime}$ & 05414000 & 4.44 & .1022 & equation & 1.3 & 3.9 \\
\hline 30 & 05414150 & Little Platte R nr Platteville & $42^{\circ} 45^{\prime} 23^{\prime \prime}$ & $90^{\circ} 29^{\prime} 40^{\prime \prime}$ & 05414000 & 54 & .1870 & relation & 22.2 & 5.6 \\
\hline 31 & 05414200 & Bear Br nr Platteville & $42^{\circ} 45^{\prime} 46^{\prime \prime}$ & $90^{\circ} 30^{\prime} 06^{\prime \prime}$ & 05414000 & 2.72 & .3770 & relation & 1.7 & 8.5 \\
\hline 32 & 05414205 & Young Br nr Platteville & $42^{\circ} 45^{\prime} 43^{\prime \prime}$ & $90^{\circ} 31^{\prime} 56^{\prime \prime}$ & 05414000 & 2.34 & .0820 & relation & .3 & 2.0 \\
\hline 33 & 05414210 & Roundtree $\mathrm{Br}$ at Platteville & $42^{\circ} 44^{\prime} 02^{\prime \prime}$ & $90^{\circ} 30^{\prime} 22^{\prime \prime}$ & 05414000 & 12.5 & .4750 & relation & 5.7 & 6.2 \\
\hline 34 & 05414220 & Little Platte R nr Dickeyville & $42^{\circ} 39^{\prime} 34^{\prime \prime}$ & $90^{\circ} 34^{\prime} 07^{\prime \prime}$ & 05414000 & 96.6 & .2270 & relation & 42.1 & 5.9 \\
\hline 35 & 05414227 & Blockhouse Ck at Platteville & $42^{\circ} 41^{\prime} 10^{\prime \prime}$ & $90^{\circ} 29^{\prime} 12^{\prime \prime}$ & 05415000 & 8.19 & .2501 & equation & 3.8 & 6.2 \\
\hline 36 & 05414242 & French Ck nr Georgetown & $42^{\circ} 38^{\prime} 25^{\prime \prime}$ & $90^{\circ} 28^{\prime} 38^{\prime \prime}$ & 05415000 & 5.76 & .1571 & equation & 2.1 & 4.9 \\
\hline 37 & 05414245 & French Ck nr Georgetown & $42^{\circ} 39^{\prime} 23^{\prime \prime}$ & $90^{\circ} 30^{\prime} 40^{\prime \prime}$ & 05415000 & 9.94 & .1497 & equation & 3.5 & 4.8 \\
\hline 38 & 05414250 & Blockhouse Ck nr Dickeyville & $42^{\circ} 39^{\prime} 22^{\prime \prime}$ & $90^{\circ} 34^{\prime} 24^{\prime \prime}$ & 05415000 & 36.3 & .2330 & relation & 16.9 & 6.3 \\
\hline 39 & 05414265 & Mcadam Br nr Dickeyville & $42^{\circ} 38^{\prime} 45^{\prime \prime}$ & $90^{\circ} 34^{\prime} 42^{\prime \prime}$ & 05414000 & 13.2 & .2665 & equation & 6.3 & 6.5 \\
\hline 40 & 05414270 & Little Platte R nr Dickeyville & $42^{\circ} 39^{\prime} 24^{\prime \prime}$ & $90^{\circ} 35^{\prime} 40^{\prime \prime}$ & 05414000 & 148 & .2168 & equation & 67.0 & 6.1 \\
\hline 41 & 05414759 & Menominee R nr Kieler & $42^{\circ} 32^{\prime} 34^{\prime \prime}$ & $90^{\circ} 36^{\prime} 09^{\prime \prime}$ & 05414000 & 12 & .1450 & relation & 3.5 & 4.0 \\
\hline 42 & 05414800 & Sinsinawa R nr Hazel Green & $42^{\circ} 32^{\prime} 02^{\prime \prime}$ & $90^{\circ} 28^{\prime} 53^{\prime \prime}$ & 05415000 & 24.9 & .2040 & relation & 9.8 & 5.3 \\
\hline 43 & 05414890 & Galena R nr Cuba City & $42^{\circ} 38^{\prime} 27^{\prime \prime}$ & $90^{\circ} 23^{\prime} 49^{\prime \prime}$ & 05415000 & 15.2 & .2704 & equation & 7.4 & 6.6 \\
\hline 44 & 05414900 & Pats Ck nr Elk Grove & $42^{\circ} 40^{\prime} 03^{\prime \prime}$ & $90^{\circ} 22^{\prime} 40^{\prime \prime}$ & 05415000 & 8.5 & .1910 & relation & 3.5 & 5.6 \\
\hline 45 & 05414910 & Galena R nr Cuba City & $42^{\circ} 38^{\prime} 03^{\prime \prime}$ & $90^{\circ} 22^{\prime} 16^{\prime \prime}$ & 05415000 & 33.2 & .2435 & equation & 15.5 & 6.3 \\
\hline 46 & 05414925 & Madden Br nr Cuba City & $42^{\circ} 37^{\prime} 53^{\prime \prime}$ & $90^{\circ} 21^{\prime} 20^{\prime \prime}$ & 05415000 & 21.9 & .2017 & equation & 9.2 & 5.7 \\
\hline 47 & 05414935 & Galena R nr Benton & $42^{\circ} 34^{\prime} 00^{\prime \prime}$ & $90^{\circ} 21^{\prime} 14^{\prime \prime}$ & 05415000 & 71 & .2240 & relation & 29.4 & 5.6 \\
\hline 48 & 05414950 & Shullsburg Br at Shullsburg & $42^{\circ} 34^{\prime} 44^{\prime \prime}$ & $90^{\circ} 14^{\prime} 26^{\prime \prime}$ & 05415000 & 7.72 & .1330 & relation & 2.8 & 4.9 \\
\hline 49 & 05414970 & Shullsburg Br at Lead Mine & $42^{\circ} 33^{\prime} 42^{\prime \prime}$ & $90^{\circ} 19^{\prime} 54^{\prime \prime}$ & 05415000 & 31.1 & .4000 & equation & 18.8 & 8.2 \\
\hline 50 & 05415038 & Scrabble Br nr Hazel Green & $42^{\circ} 30^{\prime} 52^{\prime \prime}$ & $90^{\circ} 24^{\prime} 10^{\prime \prime}$ & 05415000 & 4.69 & .2050 & relation & 1.7 & 4.9 \\
\hline
\end{tabular}

${ }^{1}$ Low-flow discharge measurements at partial-record stations were compared to one or more nearby USGS gaging stations to establish a relation between measured discharge and concurrent daily discharge. The nearby gaging station that provided the best relation with the partial-record station was chosen as the index station and was associated with nearby miscellaneous measurement sites.

${ }^{2}$ Mismatch between surface-water and ground-water drainage area. 
[USGS, U.S. Geological Survey; ID, identification number; $\mathrm{dd}^{\circ} \mathrm{mm}$ 'ss", degrees, minutes, seconds; $\mathrm{mi}^{2}$, square mile; $\mathrm{ft}^{3} / \mathrm{s} / \mathrm{mi}^{2}$, cubic foot per second per square mile; $\mathrm{ft}^{3} / \mathrm{s}$, cubic foot per second; equation, statewide regression equation; relation, established relation line with index station; R, River; Bk, Brook; Br, Branch; Fk, Fork; Ck, Creek; Tr, Tributary; nr, near; N, North; S, South; E, East; W, West;

,-- not determined in historical low-flow reports. See references for full list of low-flow reports.]

\begin{tabular}{|c|c|c|c|c|c|c|c|c|c|c|}
\hline $\begin{array}{l}\text { Map } \\
\text { number }\end{array}$ & $\begin{array}{l}\text { USGS } \\
\text { station ID }\end{array}$ & Station name & $\begin{array}{c}\text { Latitude } \\
\text { (ddºm'ss") }\end{array}$ & $\begin{array}{l}\text { Longitude } \\
\text { (ddºmm'ss") }\end{array}$ & $\begin{array}{l}\text { Index } \\
\text { station }{ }^{1}\end{array}$ & $\begin{array}{c}\text { Drainage } \\
\text { area } \\
\left(\mathrm{mi}^{2}\right)\end{array}$ & $\begin{array}{c}\text { Base flow } \\
\text { factor } \\
\left(\mathrm{ft}^{3} / \mathrm{s} / \mathrm{mi}^{2}\right)\end{array}$ & $\begin{array}{l}\text { Base flow } \\
\text { method }\end{array}$ & $\begin{array}{l}\text { Base flow } \\
\quad\left(\mathrm{ft}^{3} / \mathrm{s}\right)\end{array}$ & $\begin{array}{c}\text { Recharge } \\
\text { (inches) }\end{array}$ \\
\hline 51 & 05432050 & Pecatonica R Tr nr Livingston & $42^{\circ} 55^{\prime} 46^{\prime \prime}$ & $90^{\circ} 24^{\prime} 13^{\prime \prime}$ & 05432500 & 4.32 & 0.2450 & relation & 2.3 & 7.2 \\
\hline 52 & 05432100 & Pecatonica R nr Mineral Point & $42^{\circ} 48^{\prime} 43^{\prime \prime}$ & $90^{\circ} 15^{\prime} 30^{\prime \prime}$ & 05432500 & 68.8 & .2480 & relation & 35.6 & 7.0 \\
\hline 53 & 05432170 & Mineral Point Br nr Mineral Point & $42^{\circ} 50^{\prime} 31^{\prime \prime}$ & $90^{\circ} 14^{\prime} 07^{\prime \prime}$ & 05432500 & 32.1 & .2425 & equation & 14.9 & 6.3 \\
\hline 54 & 05432207 & E Pecatonica R Tr nr Linden & $42^{\circ} 55^{\prime} 13^{\prime \prime}$ & $90^{\circ} 15^{\prime} 42^{\prime \prime}$ & 05432500 & 2.03 & .1970 & relation & 1.3 & 8.7 \\
\hline 55 & 05432219 & Pedler Ck nr Linden & $42^{\circ} 54^{\prime} 27^{\prime \prime}$ & $90^{\circ} 14^{\prime} 59^{\prime \prime}$ & 05432500 & 13.6 & .2290 & relation & 7.5 & 7.5 \\
\hline 56 & 05432230 & E Pecatonica R nr Mifflin & $42^{\circ} 51^{\prime} 18^{\prime \prime}$ & $90^{\circ} 17^{\prime} 12^{\prime \prime}$ & 05432500 & 39.8 & .2095 & equation & 17.2 & 5.9 \\
\hline 57 & 05432270 & Brewery Ck at Mineral Point & $42^{\circ} 51^{\prime} 13^{\prime \prime}$ & $90^{\circ} 10^{\prime} 38^{\prime \prime}$ & 05432500 & 6.74 & .2220 & relation & 3.3 & 6.7 \\
\hline 58 & 05432300 & Rock Br nr Mineral Point & $42^{\circ} 50^{\prime} 02^{\prime \prime}$ & $90^{\circ} 09^{\prime} 15^{\prime \prime}$ & 05432500 & 4.83 & .2480 & relation & 3.0 & 8.4 \\
\hline 59 & 05432350 & Bonner $\mathrm{Br}$ at Belmont & $42^{\circ} 44^{\prime} 02^{\prime \prime}$ & $90^{\circ} 19^{\prime} 42^{\prime \prime}$ & 05432500 & 5.62 & .2580 & relation & 2.9 & 7.0 \\
\hline 60 & 05432400 & Bonner $\mathrm{Br}$ at Calamine & $42^{\circ} 44^{\prime} 31^{\prime \prime}$ & $90^{\circ} 10^{\prime} 15^{\prime \prime}$ & 05432500 & 34.5 & .3232 & equation & 18.7 & 7.4 \\
\hline 61 & 05432410 & Pecatonica $\mathrm{R}$ at Calamine & $42^{\circ} 44^{\prime} 28^{\prime \prime}$ & $90^{\circ} 10^{\prime} 08^{\prime \prime}$ & 05432500 & 232 & .2543 & equation & 115.1 & 6.7 \\
\hline 62 & 05432508 & Otter Ck nr Waldwick & $42^{\circ} 47^{\prime} 49^{\prime \prime}$ & $90^{\circ} 06^{\prime} 28^{\prime \prime}$ & 05432500 & 8.36 & .3830 & relation & 6.4 & 10.4 \\
\hline 63 & 05432512 & Otter Ck Tr nr Waldwick & $42^{\circ} 47^{\prime} 58^{\prime \prime}$ & $90^{\circ} 05^{\prime} 48^{\prime \prime}$ & 05432500 & 2.36 & .2948 & equation & 1.1 & 6.6 \\
\hline 64 & 05432514 & Otter Ck nr Fayette & $42^{\circ} 46^{\prime} 13^{\prime \prime}$ & $90^{\circ} 05^{\prime} 43^{\prime \prime}$ & 05432500 & 19.8 & .3069 & equation & 10.3 & 7.1 \\
\hline 65 & 05432518 & Otter Ck nr Fayette & $42^{\circ} 43^{\prime} 06^{\prime \prime}$ & $90^{\circ} 04^{\prime} 40^{\prime \prime}$ & 05432500 & 30.6 & .2780 & equation & 15.3 & 6.8 \\
\hline 66 & 05432520 & Otter Ck Tr nr Fayette & $42^{\circ} 43^{\prime} 06^{\prime \prime}$ & $90^{\circ} 04^{\prime} 34^{\prime \prime}$ & 05432500 & 5.25 & .0640 & equation & 1.2 & 3.1 \\
\hline 67 & 05432522 & Otter Ck Tr nr Darlington & $42^{\circ} 41^{\prime} 15^{\prime \prime}$ & $90^{\circ} 03^{\prime} 40^{\prime \prime}$ & 05432500 & 8.21 & .1750 & relation & 5.6 & 9.3 \\
\hline 68 & 05432525 & Otter Ck nr Darlington & $42^{\circ} 40^{\prime} 32^{\prime \prime}$ & $90^{\circ} 03^{\prime} 51^{\prime \prime}$ & 05432500 & 49.3 & .2420 & relation & 24.3 & 6.7 \\
\hline 69 & 05432550 & Ames Br nr Darlington & $42^{\circ} 39^{\prime} 26^{\prime \prime}$ & $90^{\circ} 05^{\prime} 34^{\prime \prime}$ & 05432500 & 44.3 & .2610 & relation & 23.8 & 7.3 \\
\hline 70 & 05432600 & Wolf Ck at Gratiot & $42^{\circ} 34^{\prime} 49^{\prime \prime}$ & $90^{\circ} 01 ' 27^{\prime \prime}$ & 05432500 & 27.6 & .1740 & relation & 18.8 & 9.3 \\
\hline 71 & 05432645 & Spafford Ck nr S Wayne & $42^{\circ} 33^{\prime} 30^{\prime \prime}$ & $89^{\circ} 53^{\prime} 51^{\prime \prime}$ & 05432500 & 43.4 & .2410 & relation & 23.1 & 7.2 \\
\hline 72 & 05432740 & Pecatonica R nr Hollandale & $42^{\circ} 53^{\prime} 11^{\prime \prime}$ & $89^{\circ} 54^{\prime} 26^{\prime \prime}$ & 05433000 & 52.8 & .3097 & equation & 28.2 & 7.3 \\
\hline 73 & 05432800 & Dodge $\mathrm{Br}$ at Hollandale & $42^{\circ} 52^{\prime} 49^{\prime \prime}$ & $89^{\circ} 56^{\prime} 18^{\prime \prime}$ & 05432500 & 66 & .3440 & relation & 40.5 & 8.3 \\
\hline 74 & 05432900 & Blue Mounds Br nr Hollandale & $42^{\circ} 52^{\prime} 46^{\prime \prime}$ & $89^{\circ} 51^{\prime} 38^{\prime \prime}$ & 05433000 & 31 & .3782 & equation & 18.2 & 8.0 \\
\hline 75 & 05432924 & $\begin{array}{l}\text { Pleasant Vallet } \mathrm{Br} \text { Tr nr Blanchard- } \\
\text { ville }\end{array}$ & $42^{\circ} 52^{\prime} 30^{\prime \prime}$ & $89^{\circ} 47^{\prime} 13^{\prime \prime}$ & 05433000 & 9.83 & .6977 & equation & 7.7 & 10.7 \\
\hline
\end{tabular}

${ }^{1}$ Low-flow discharge measurements at partial-record stations were compared to one or more nearby USGS gaging stations to establish a relation between measured discharge and concurrent daily discharge. The nearby gaging station that provided the best relation with the partial-record station was chosen as the index station and was associated with nearby miscellaneous measurement sites.

${ }^{2}$ Mismatch between surface-water and ground-water drainage area. 
Appendix 11D. Average annual base flow and corresponding recharge, 1970-99, for partial-record stations in the Pecatonica-Sugar River Basin—Continued.

[USGS, U.S. Geological Survey; ID, identification number; $\mathrm{dd}^{\circ} \mathrm{mm}$ 'ss", degrees, minutes, seconds; $\mathrm{mi}^{2}$, square mile; $\mathrm{ft}^{3} / \mathrm{s} / \mathrm{mi}^{2}$, cubic foot per second per square mile; $\mathrm{ft}^{3} / \mathrm{s}$, cubic foot per second; equation, statewide regression equation; relation, established relation line with index station; R, River; Bk, Brook; Br, Branch; Fk, Fork; Ck, Creek; Tr, Tributary; nr, near; N, North; S, South; E, East; W, West; --, not determined in historical low-flow reports. See references for full list of low-flow reports.]

\begin{tabular}{|c|c|c|c|c|c|c|c|c|c|c|}
\hline $\begin{array}{c}\text { Map } \\
\text { number }\end{array}$ & $\begin{array}{c}\text { USGS } \\
\text { station ID }\end{array}$ & Station name & $\begin{array}{c}\text { Latitude } \\
\text { (dd }{ }^{\circ} \mathrm{mm} \text { 'ss") }\end{array}$ & $\begin{array}{c}\text { Longitude } \\
\text { (dd }{ }^{\circ} \mathrm{mm} \text { 'ss") }\end{array}$ & $\begin{array}{l}\text { Index } \\
\text { station }^{1}\end{array}$ & $\begin{array}{c}\text { Drainage } \\
\text { area } \\
\left(\mathrm{mi}^{2}\right)\end{array}$ & $\begin{array}{c}\text { Base flow } \\
\text { factor } \\
\left(\mathrm{ft}^{3} / \mathrm{s} / \mathrm{mi}^{2}\right)\end{array}$ & $\begin{array}{c}\text { Base flow } \\
\text { method }\end{array}$ & $\begin{array}{c}\text { Base flow } \\
\qquad\left(\mathrm{ft}^{3} / \mathrm{s}\right)\end{array}$ & $\begin{array}{c}\text { Recharge } \\
\text { (inches) }\end{array}$ \\
\hline 76 & 05432928 & $\begin{array}{l}\text { Pleasant Valley } \mathrm{Br} \mathrm{nr} \\
\text { Blanchardville }\end{array}$ & $42^{\circ} 52^{\prime} 13^{\prime \prime}$ & $89^{\circ} 48^{\prime} 33^{\prime \prime}$ & 05433000 & 21.5 & 0.5345 & equation & 15.0 & 9.5 \\
\hline 77 & 05432940 & Pleasant Valley Br nr Hollandale & $42^{\circ} 51^{\prime} 54^{\prime \prime}$ & $89^{\circ} 51^{\prime} 26^{\prime \prime}$ & 05433000 & 33 & .3349 & equation & 18.2 & 7.5 \\
\hline 78 & 05433200 & Sawmill Ck nr Blanchardville & $42^{\circ} 45^{\prime} 46^{\prime \prime}$ & $89^{\circ} 51^{\prime} 33^{\prime \prime}$ & 05433000 & 21.9 & .3931 & equation & 13.0 & 8.1 \\
\hline 79 & 05433400 & Yellowstone R nr Waldwick & $42^{\circ} 48^{\prime} 01^{\prime \prime}$ & $89^{\circ} 58^{\prime} 38^{\prime \prime}$ & 05433000 & 18.7 & .1430 & equation & 6.5 & 4.8 \\
\hline 80 & 05433620 & Dougherty $\mathrm{Ck}$ at Argyle & $42^{\circ} 41^{\prime} 53^{\prime \prime}$ & $89^{\circ} 51^{\prime} 18^{\prime \prime}$ & 05433000 & 27.1 & .3545 & equation & 15.3 & 7.7 \\
\hline 81 & 05433700 & Whiteside Ck nr Argyle & $43^{\circ} 40^{\prime} 10^{\prime \prime}$ & $89^{\circ} 53^{\prime} 13^{\prime \prime}$ & 05432500 & 19.9 & .4100 & relation & 17.0 & 11.6 \\
\hline 82 & 05434260 & Skinner Ck nr Browntown & $42^{\circ} 35^{\prime} 32^{\prime \prime}$ & $89^{\circ} 46^{\prime} 30^{\prime \prime}$ & 05432500 & 47.3 & .2880 & equation & 24.3 & 7.0 \\
\hline 83 & 05434300 & Jordan Ck nr Browntown & $42^{\circ} 36^{\prime} 38^{\prime \prime}$ & $89^{\circ} 47^{\prime} 08^{\prime \prime}$ & 05432500 & 14.3 & .3410 & relation & 7.7 & 7.3 \\
\hline 84 & 05434317 & Skinner Ck at Browntown & $42^{\circ} 34^{\prime} 48^{\prime \prime}$ & $89^{\circ} 48^{\prime} 09^{\prime \prime}$ & 05432500 & 71 & .3030 & relation & 34.0 & 6.5 \\
\hline 85 & 05435030 & Honey Ck nr Monroe & $42^{\circ} 35^{\prime} 54^{\prime \prime}$ & $89^{\circ} 40^{\prime} 05^{\prime \prime}$ & 05432500 & 4.74 & .5740 & relation & 3.3 & 9.5 \\
\hline 86 & 05435100 & Honey Ck nr Martintown & $42^{\circ} 30^{\prime} 51^{\prime \prime}$ & $89^{\circ} 46^{\prime} 03^{\prime \prime}$ & 05432500 & 32.8 & -- & relation & 16.3 & 6.8 \\
\hline 87 & 05435290 & Richland Ck nr Monroe & $42^{\circ} 33^{\prime} 41^{\prime \prime}$ & $89^{\circ} 34^{\prime} 39^{\prime \prime}$ & 05436500 & 10.9 & .2880 & relation & 5.6 & 7.0 \\
\hline 88 & 05435300 & Twin Grove Br nr Monroe & $42^{\circ} 31^{\prime} 47^{\prime \prime}$ & $89^{\circ} 34^{\prime} 22^{\prime \prime}$ & 05436500 & 6.43 & .2500 & relation & 2.7 & 5.7 \\
\hline 89 & 05435325 & Richland Ck at Clarno & $42^{\circ} 31^{\prime} 02^{\prime \prime}$ & $89^{\circ} 38^{\prime} 46^{\prime \prime}$ & 05436500 & 39.3 & .2720 & equation & 19.5 & 6.7 \\
\hline 90 & 05435900 & Sugar R Tr nr Pine Bluff & $43^{\circ} 02^{\prime} 48^{\prime \prime}$ & $89^{\circ} 38^{\prime} 42^{\prime \prime}$ & 05436500 & 7.42 & .0261 & equation & 1.1 & 1.9 \\
\hline 91 & 05435910 & Sugar R at Riley & $43^{\circ} 01^{\prime} 31^{\prime \prime}$ & $89^{\circ} 37^{\prime} 18^{\prime \prime}$ & 05436500 & 14.2 & .2852 & equation & 7.1 & 6.8 \\
\hline 92 & 05435936 & Badger Mill Ck nr Verona & $42^{\circ} 59^{\prime} 31^{\prime \prime}$ & $89^{\circ} 30^{\prime} 49^{\prime \prime}$ & 05436500 & 14.9 & .0207 & equation & 1.9 & 1.7 \\
\hline 93 & 05435943 & Badger Mill $\mathrm{Ck}$ at Verona & $42^{\circ} 58^{\prime} 38^{\prime \prime}$ & $89^{\circ} 32^{\prime} 21^{\prime \prime}$ & 05436500 & 20.3 & .1770 & relation & 7.0 & 4.7 \\
\hline 94 & 05435950 & Sugar R nr Verona & $42^{\circ} 56^{\prime} 58^{\prime \prime}$ & $89^{\circ} 32^{\prime} 40^{\prime \prime}$ & 05436500 & 82.7 & .1940 & relation & 33.1 & 5.4 \\
\hline 95 & 05435959 & Sugar R at Paoli & $42^{\circ} 56^{\prime} 01^{\prime \prime}$ & $89^{\circ} 31^{\prime} 35^{\prime \prime}$ & 05436500 & 88.7 & .2580 & relation & 35.2 & 5.4 \\
\hline 96 & 05435970 & W Br Sugar R nr Mt Horeb & $42^{\circ} 58^{\prime} 28^{\prime \prime}$ & $89^{\circ} 44^{\prime} 05^{\prime \prime}$ & 05436500 & 2.5 & .6048 & equation & 1.8 & 9.7 \\
\hline 97 & 05435980 & W Br Sugar R nr Mt Vernon & $42^{\circ} 54^{\prime} 47^{\prime \prime}$ & $89^{\circ} 37^{\prime} 19^{\prime \prime}$ & 05436500 & 32.7 & -- & relation & 16.2 & 6.7 \\
\hline 98 & 05435983 & Fryes Feeder nr Mt. Vernon & $42^{\circ} 57^{\prime} 46^{\prime \prime}$ & $89^{\circ} 40^{\prime} 28^{\prime \prime}$ & 05436500 & 4.56 & .2879 & equation & 2.2 & 6.6 \\
\hline 99 & 05435991 & Mt Vernon Ck at Mt Vernon & $42^{\circ} 57^{\prime} 15^{\prime \prime}$ & $89^{\circ} 40^{\prime} 04^{\prime \prime}$ & 05436500 & 9.27 & .3250 & equation & 4.9 & 7.2 \\
\hline 100 & 05435993 & Mt Vernon Ck at Mt Vernon & $42^{\circ} 57^{\prime} 15^{\prime \prime}$ & $89^{\circ} 40^{\prime} 01^{\prime \prime}$ & 05436500 & 9.29 & 1.4151 & equation & 10.6 & ${ }^{2} 15.4$ \\
\hline
\end{tabular}

${ }^{1}$ Low-flow discharge measurements at partial-record stations were compared to one or more nearby USGS gaging stations to establish a relation between measured discharge and concurrent daily discharge. The nearby gaging station that provided the best relation with the partial-record station was chosen as the index station and was associated with nearby miscellaneous measurement sites.

${ }^{2}$ Mismatch between surface-water and ground-water drainage area. 
[USGS, U.S. Geological Survey; ID, identification number; $\mathrm{dd}^{\circ} \mathrm{mm}$ 'ss", degrees, minutes, seconds; $\mathrm{mi}^{2}$, square mile; $\mathrm{ft}^{3} / \mathrm{s} / \mathrm{mi}^{2}$, cubic foot per second per square mile; $\mathrm{ft}^{3} / \mathrm{s}$, cubic foot per second; equation, statewide regression equation; relation, established relation line with index station; R, River; Bk, Brook; Br, Branch; Fk, Fork; Ck, Creek; Tr, Tributary; nr, near; N, North; S, South; E, East; W, West;

--, not determined in historical low-flow reports. See references for full list of low-flow reports.]

\begin{tabular}{|c|c|c|c|c|c|c|c|c|c|c|}
\hline $\begin{array}{c}\text { Map } \\
\text { number }\end{array}$ & $\begin{array}{c}\text { USGS } \\
\text { station ID }\end{array}$ & Station name & $\begin{array}{c}\text { Latitude } \\
\text { (dd }{ }^{\circ} \mathrm{mm} \text { 'ss") }\end{array}$ & $\begin{array}{l}\text { Longitude } \\
\text { (ddºm'ss") }\end{array}$ & $\begin{array}{l}\text { Index } \\
\text { station }^{1}\end{array}$ & $\begin{array}{c}\text { Drainage } \\
\text { area } \\
\left(\mathrm{mi}^{2}\right)\end{array}$ & $\begin{array}{c}\text { Base flow } \\
\text { factor } \\
\left(\mathrm{ft}^{3} / \mathrm{s} / \mathrm{mi}^{2}\right)\end{array}$ & $\begin{array}{c}\text { Base flow } \\
\text { method }\end{array}$ & $\begin{array}{c}\text { Base flow } \\
\qquad\left(\mathrm{ft}^{3} / \mathrm{s}\right)\end{array}$ & $\begin{array}{r}\text { Recharge } \\
\text { (inches) }\end{array}$ \\
\hline 101 & 05436076 & Sugar R at Belleville & $42^{\circ} 51^{\prime} 40^{\prime \prime}$ & $89^{\circ} 32^{\prime} 01^{\prime \prime}$ & 05436500 & 172 & 0.4260 & relation & 94.3 & 7.4 \\
\hline 102 & 05436190 & Sugar R nr Dayton & $42^{\circ} 47^{\prime} 59^{\prime \prime}$ & $89^{\circ} 29^{\prime} 12^{\prime \prime}$ & 05436500 & 233 & .3334 & equation & 133.1 & 7.8 \\
\hline 103 & 05436200 & Gill Ck nr Bklyn & $42^{\circ} 49^{\prime} 38^{\prime \prime}$ & $89^{\circ} 26^{\prime} 43^{\prime \prime}$ & 05436500 & 3.33 & .2070 & relation & 1.2 & 4.9 \\
\hline 104 & 05436215 & Allen Ck at Bklyn & $42^{\circ} 50^{\prime} 24^{\prime \prime}$ & $89^{\circ} 22^{\prime} 09^{\prime \prime}$ & 05436500 & 10.8 & .1320 & relation & 2.5 & 3.1 \\
\hline 105 & 05436225 & Allen Ck at Evansville & $42^{\circ} 46^{\prime} 30^{\prime \prime}$ & $89^{\circ} 17^{\prime} 34^{\prime \prime}$ & 05436500 & 27.5 & .1280 & relation & 9.6 & 4.7 \\
\hline 106 & 05436250 & Allen Ck nr Albany & $42^{\circ} 44^{\prime} 11^{\prime \prime}$ & $89^{\circ} 25^{\prime} 57^{\prime \prime}$ & 05436500 & 70.5 & .2570 & relation & 46.8 & 9.0 \\
\hline 107 & 05436270 & Little Sugar R nr New Glarus & $42^{\circ} 50^{\prime} 39^{\prime \prime}$ & $89^{\circ} 39^{\prime} 24^{\prime \prime}$ & 05436500 & 10.5 & .4950 & equation & 6.9 & 9.0 \\
\hline 108 & 05436281 & Little Sugar R at New Glarus & $42^{\circ} 48^{\prime} 52^{\prime \prime}$ & $89^{\circ} 37^{\prime} 49^{\prime \prime}$ & 05436500 & 22.4 & .3930 & relation & 13.0 & 7.9 \\
\hline 109 & 05436285 & Legler School Br at New Glarus & $42^{\circ} 48^{\prime} 13^{\prime \prime}$ & $89^{\circ} 38^{\prime} 07^{\prime \prime}$ & 05436500 & 4.01 & .3872 & equation & 2.3 & 7.7 \\
\hline 110 & 05436300 & Little Sugar R nr New Glarus & $42^{\circ} 47^{\prime} 11^{\prime \prime}$ & $89^{\circ} 35^{\prime} 21^{\prime \prime}$ & 05436500 & 39.6 & .3760 & relation & 24.5 & 8.4 \\
\hline 111 & 05436360 & W Br Litle Sugar R at Monticello & $42^{\circ} 44^{\prime} 46^{\prime \prime}$ & $89^{\circ} 35^{\prime} 57^{\prime \prime}$ & 05436500 & 29 & .4409 & equation & 18.4 & 8.6 \\
\hline 112 & 05436362 & W Br Little Sugar R at Monticello & $42^{\circ} 44^{\prime} 43^{\prime \prime}$ & $89^{\circ} 35^{\prime} 02^{\prime \prime}$ & 05436500 & 33.6 & .4460 & relation & 21.1 & 8.5 \\
\hline 113 & 05436380 & Burgy Ck nr Monticello & $42^{\circ} 42^{\prime} 52^{\prime \prime}$ & $89^{\circ} 35^{\prime} 56^{\prime \prime}$ & 05436500 & 14 & .2568 & equation & 6.6 & 6.4 \\
\hline 114 & 05436400 & Little Sugar R nr Monticello & $42^{\circ} 43^{\prime} 30^{\prime \prime}$ & $89^{\circ} 31^{\prime} 44^{\prime \prime}$ & 05436500 & 118 & .3490 & relation & 67.6 & 7.8 \\
\hline 115 & 05436440 & Searles Ck nr Brodhead & $42^{\circ} 38^{\prime} 43^{\prime \prime}$ & $89^{\circ} 26^{\prime} 47^{\prime \prime}$ & 05436500 & 19.3 & .0659 & equation & 4.5 & 3.2 \\
\hline 116 & 05436510 & Sylvester Ck nr Monroe & $42^{\circ} 38^{\prime} 03^{\prime \prime}$ & $89^{\circ} 34^{\prime} 21^{\prime \prime}$ & 04073500 & 6.06 & .3300 & relation & 3.7 & 8.3 \\
\hline 117 & 05436542 & Juda Br at Juda & $42^{\circ} 35^{\prime} 30^{\prime \prime}$ & $89^{\circ} 29^{\prime} 43^{\prime \prime}$ & 05436500 & 8.13 & .3570 & relation & 3.6 & 6.0 \\
\hline 118 & 05436600 & Sylvester Cr nr Brodhead & $42^{\circ} 36^{\prime} 07^{\prime \prime}$ & $89^{\circ} 24^{\prime} 51^{\prime \prime}$ & 05436500 & 44.7 & .2880 & relation & 24.4 & 7.4 \\
\hline 119 & 05436700 & Taylor Ck nr Brodhead & $42^{\circ} 35^{\prime} 35^{\prime \prime}$ & $89^{\circ} 21^{\prime} 29^{\prime \prime}$ & 05436500 & 26.1 & .1630 & relation & 11.1 & 5.8 \\
\hline
\end{tabular}

${ }^{1}$ Low-flow discharge measurements at partial-record stations were compared to one or more nearby USGS gaging stations to establish a relation between measured discharge and concurrent daily discharge. The nearby gaging station that provided the best relation with the partial-record station was chosen as the index station and was associated with nearby miscellaneous measurement sites.

${ }^{2}$ Mismatch between surface-water and ground-water drainage area. 


\section{Rock-Fox River Basin}
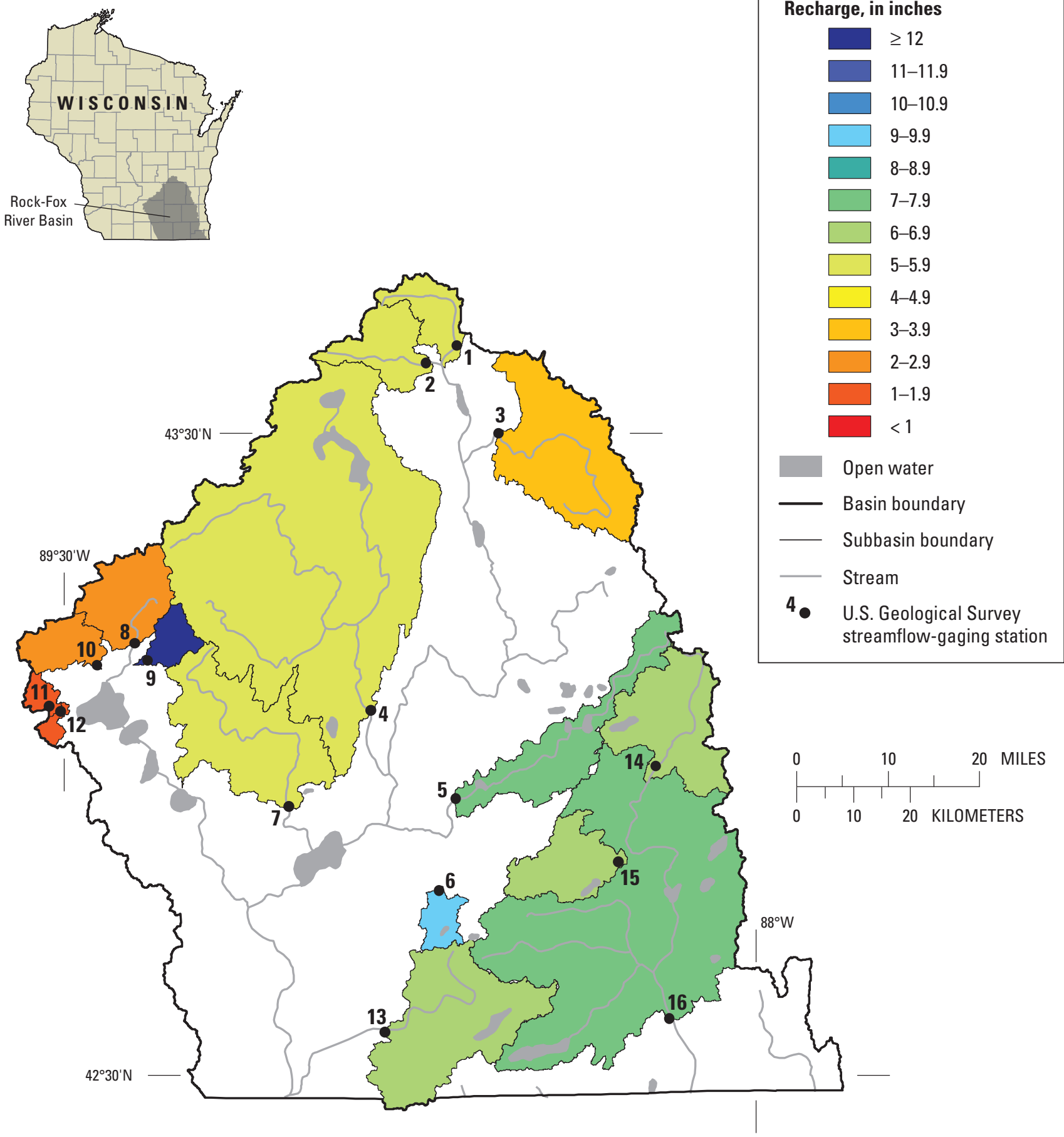

Appendix 12-A. Locations of streamflow-gaging stations in the Rock-Fox River Basin and spatial distribution of average annual recharge.

\section{EXPLANATION}

Recharge, in inches

\begin{tabular}{|l|l}
\hline & $\geq 12$ \\
\hline & $11-11.9$ \\
\hline & $10-10.9$ \\
\hline & $9-9.9$ \\
\hline & $8-8.9$ \\
\hline & $7-7.9$ \\
\hline & $6-6.9$ \\
\hline & $5-5.9$ \\
\hline & $4-4.9$ \\
\hline & $3-3.9$ \\
\hline & $2-2.9$ \\
\hline & $1-1.9$ \\
\hline & $<1$
\end{tabular}

Open water

Stream

J.S. Geological Survey streamflow-gaging station 

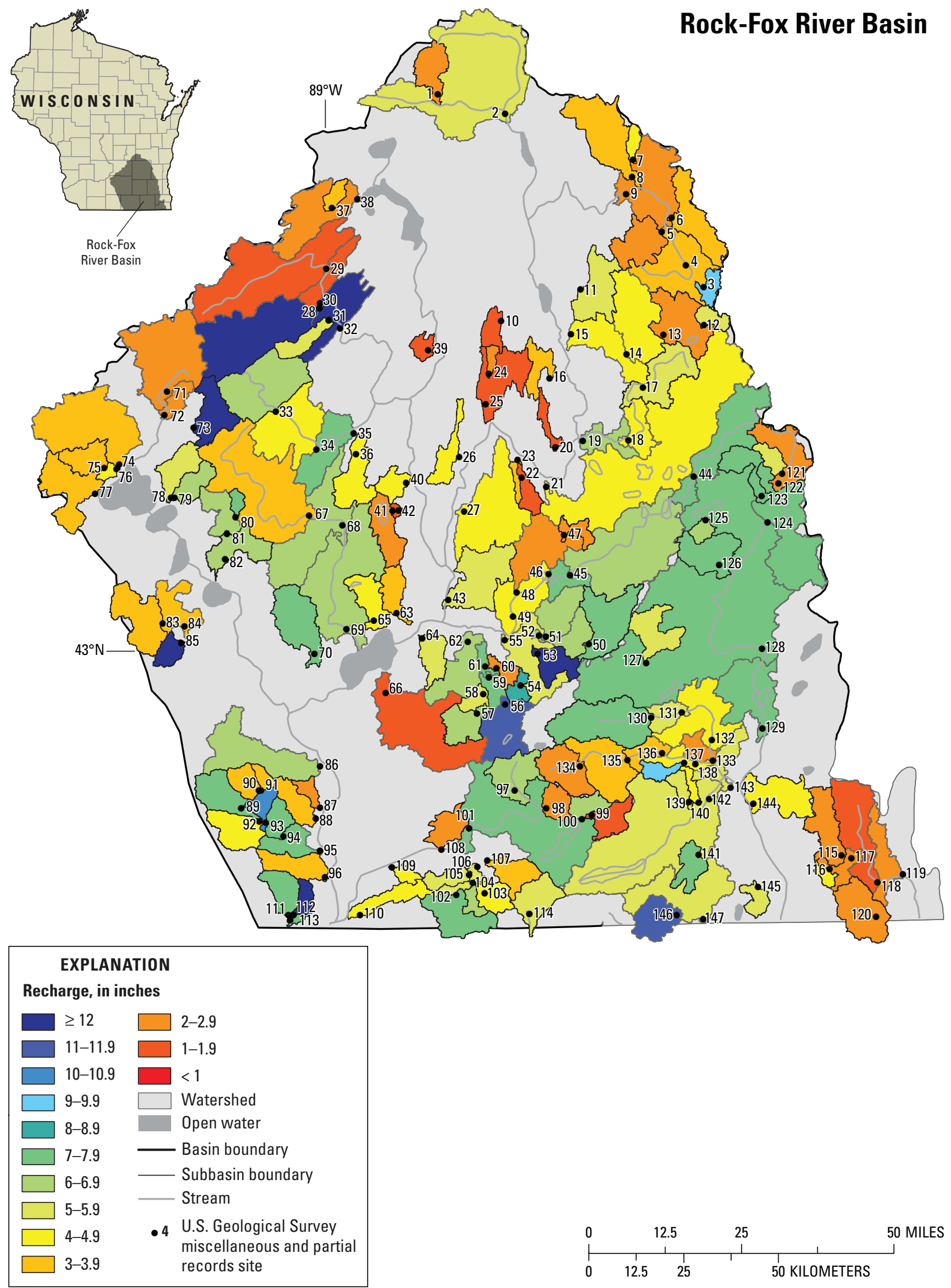

Appendix 12-B. Locations of miscellaneous and partial-record sites in the Rock-Fox River Basin and spatial distribution of estimated average annual recharge. 
Appendix 12C. Average annual base flow and corresponding recharge, 1970-99, for streamflow-gaging stations in the Rock-Fox River Basin.

[USGS, U.S. Geological Survey; ID, identification number; mi² $^{2}$, square mile; $\mathrm{ft}^{3} / \mathrm{s}$, cubic foot per second; BFI, Baseflow Index; ex rec, extended record; R, River; Bk, Brook; Br, Branch; Fk, Fork; Ck, Creek; Tr, Tributary; nr, near; N, North; S, South; E, East; W, West; --, not determined in historical low-flow reports. See references for full list of low-flow reports.]

\begin{tabular}{|c|c|c|c|c|c|c|c|c|c|c|c|}
\hline $\begin{array}{c}\text { Map } \\
\text { number }\end{array}$ & $\begin{array}{l}\text { USGS } \\
\text { station } \\
\text { ID }\end{array}$ & Station name & $\begin{array}{c}\text { Drainage } \\
\text { area } \\
\left(\mathrm{mi}^{2}\right)\end{array}$ & $\begin{array}{c}\text { Period of } \\
\text { analysis } \\
\text { (water years') }\end{array}$ & $\begin{array}{c}\text { Base flow } \\
\text { method }\end{array}$ & $\begin{array}{l}\text { Base flow } \\
\text { index }\end{array}$ & $\begin{array}{c}\text { Base flow } \\
\quad\left(\mathrm{ft}^{3} / \mathrm{s}\right)\end{array}$ & $\begin{array}{c}\text { Recharge } \\
\text { (inches) }\end{array}$ & $\begin{array}{c}\text { Total } \\
\text { streamflow } \\
\left(\mathrm{ft}^{3} / \mathrm{s}\right)\end{array}$ & $\begin{array}{c}\text { Total } \\
\text { streamflow } \\
\text { (inches) }\end{array}$ & $\begin{array}{l}{ }^{2} 090 \\
\left(\mathrm{ft}^{3} / \mathrm{s}\right)\end{array}$ \\
\hline 1 & 05423000 & W Br Rock R nr Waupun & 40.7 & $1950-1981$ & ex rec & -- & 17 & 5.7 & -- & -- & 3.2 \\
\hline 2 & 05423500 & S Br Rock R at Waupun & 63.6 & 1970-1999 & ex rec & -- & 25 & 5.3 & -- & -- & 9.5 \\
\hline 3 & 05424000 & E Br Rock R nr Mayville & 179 & $1950-1970$ & ex rec & -- & 50 & 3.8 & -- & -- & 18 \\
\hline 4 & 05426000 & Crawfish $\mathrm{R}$ at Milford & 762 & 1970-1999 & BFI & 0.6010 & 320 & 5.8 & 530 & 9.4 & 87 \\
\hline 5 & 05426250 & Bark R nr Rome & 122 & 1980-1999 & ex rec & -- & 66 & 7.4 & -- & -- & 28 \\
\hline 6 & 05427000 & Whitewater Ck at Whitewater & 22.8 & $\begin{array}{c}1927, \\
1944-1954\end{array}$ & ex rec & -- & 16 & 9.5 & -- & -- & 11 \\
\hline 7 & 05427507 & Koshkonong Ck nr Rockdale & 150 & $1978-1982$ & ex rec & -- & 62 & 5.6 & -- & -- & 28 \\
\hline 8 & 05427718 & Yahara R at Windsor & 73.6 & $1970-1999$ & ex rec & -- & 15 & 2.7 & -- & -- & 9.2 \\
\hline 9 & 05427800 & Token Ck nr Madison & 24.3 & $1976-1980$ & ex rec & -- & 22 & 12.3 & -- & -- & 16 \\
\hline 10 & 05427900 & Sixmile Ck nr Waunakee & 41.1 & $1977-1981$ & ex rec & -- & 8.2 & 2.7 & -- & -- & 4.5 \\
\hline 11 & 05427943 & Pheasant Br nr Middleton & 9.61 & $1978-1981$ & ex rec & -- & 1.0 & 1.4 & -- & -- & -- \\
\hline 12 & 05427948 & Pheasant $\mathrm{Br}$ at Middleton & 18.3 & $1975-1999$ & BFI & .3460 & 1.5 & 1.1 & 5.0 & 3.7 & .80 \\
\hline 13 & 05431486 & Turtle Ck nr Clinton & 2.57 & 1970-1999 & BFI & .6720 & 97 & 6.6 & 50 & 10.0 & 56 \\
\hline 14 & 05543830 & Fox R at Waukesha & 126 & $1970-1999$ & BFI & .5730 & 64 & 6.9 & 110 & 12.3 & 28 \\
\hline 15 & 05544200 & Mukwonago R at Mukwonago & 74.1 & 1974-1998 & BFI & .6050 & 33 & 6.0 & 55 & 10.1 & 22 \\
\hline 16 & 05545750 & Fox R at New Munster & 811 & $1970-1998$ & BFI & .6260 & 420 & 7.1 & 670 & 11.3 & 190 \\
\hline
\end{tabular}

${ }^{1}$ Water year is a 12-month period from October 1 through September 30, and is designated by the calendar year in which it ends (for example, the 2004 water year occurred October 1,2003 through September 30, 2004).

${ }^{2}$ Discharge that is exceeded 90 percent of the time. 
[USGS, U.S. Geological Survey; ID, identification number; $\mathrm{dd}^{\circ} \mathrm{mm}$ 'ss", degrees, minutes, seconds; $\mathrm{mi}^{2}$, square mile; $\mathrm{ft}^{3} / \mathrm{s} / \mathrm{mi}^{2}$, cubic foot per second per square mile; $\mathrm{ft}^{3} / \mathrm{s}$, cubic foot per second; equation, statewide regression equation; relation, established relation line with index station; R, River; Bk, Brook; Br, Branch; Fk, Fork; Ck, Creek; Tr, Tributary; nr, near; N, North; S, South; E, East; W, West;

--, not determined in historical low-flow reports. See references for full list of low-flow reports.]

\begin{tabular}{|c|c|c|c|c|c|c|c|c|c|c|}
\hline $\begin{array}{c}\text { Map } \\
\text { number }\end{array}$ & $\begin{array}{c}\text { USGS } \\
\text { station ID }\end{array}$ & Station name & $\begin{array}{c}\text { Latitude } \\
\text { (dd }{ }^{\circ} \mathrm{mm} \text { 'ss") }\end{array}$ & $\begin{array}{l}\text { Longitude } \\
\text { (ddºmm'ss") }\end{array}$ & $\begin{array}{l}\text { Index } \\
\text { station }^{1}\end{array}$ & $\begin{array}{c}\text { Drainage } \\
\text { area } \\
\left(\mathrm{mi}^{2}\right)\end{array}$ & $\begin{array}{c}\text { Base flow } \\
\text { factor } \\
\left(\mathrm{ft}^{3} / \mathrm{s} / \mathrm{mi}^{2}\right)\end{array}$ & $\begin{array}{l}\text { Base flow } \\
\text { method }\end{array}$ & $\begin{array}{c}\text { Base flow } \\
\left(\mathrm{ft}^{3} / \mathrm{s}\right)\end{array}$ & $\begin{array}{c}\text { Recharge } \\
\text { (inches) }\end{array}$ \\
\hline 1 & 05423300 & S Branch Rock R Tr nr Waupun & $43^{\circ} 39^{\prime} 46^{\prime \prime}$ & $88^{\circ} 48^{\prime} 55^{\prime \prime}$ & 05423500 & 12.6 & -- & relation & 2.2 & 2.4 \\
\hline 2 & 05423510 & W Branch Rock R nr Waupun & $43^{\circ} 38^{\prime} 04^{\prime \prime}$ & $88^{\circ} 41^{\prime} 08^{\prime \prime}$ & 05423500 & 113 & 0.1824 & equation & 46.5 & 5.6 \\
\hline 3 & 05423800 & E Branch Rock R Tr nr Slinger & $43^{\circ} 23^{\prime} 06^{\prime \prime}$ & $88^{\circ} 18^{\prime} 29^{\prime \prime}$ & 04086500 & 4.42 & 1.6090 & relation & 3.1 & 9.5 \\
\hline 4 & 05423825 & E Branch Rock R at Allenton & $43^{\circ} 25^{\prime} 01^{\prime \prime}$ & $88^{\circ} 20^{\prime} 29^{\prime \prime}$ & 05424000 & 28.1 & -- & relation & 7.1 & 3.4 \\
\hline 5 & 05423840 & E Branch Rock R Tr \#2 nr Nenno & $43^{\circ} 27^{\prime} 51^{\prime \prime}$ & $88^{\circ} 23^{\prime} 15^{\prime \prime}$ & 05424000 & 17.3 & .0579 & equation & 3.8 & 3.0 \\
\hline 6 & 05423850 & Kohlsville R nr Kohlsville & $43^{\circ} 29^{\prime} 02^{\prime \prime}$ & $88^{\circ} 22^{\prime} 03^{\prime \prime}$ & 05424000 & 20 & .0686 & equation & 4.8 & 3.2 \\
\hline 7 & 05423890 & E Branch Rock R Tr nr Lomira & $43^{\circ} 34^{\prime} 00^{\prime \prime}$ & $88^{\circ} 26^{\prime} 24^{\prime \prime}$ & 05424000 & 3.7 & -- & relation & 1.3 & 4.8 \\
\hline 8 & 05423947 & Kummel Ck nr Theresa & $43^{\circ} 32^{\prime} 32^{\prime \prime}$ & $88^{\circ} 26^{\prime} 33^{\prime \prime}$ & 05424000 & 28.7 & .0617 & equation & 6.5 & 3.1 \\
\hline 9 & 05423960 & E Branch Rock R at Theresa & $43^{\circ} 31^{\prime} 08^{\prime \prime}$ & $88^{\circ} 27^{\prime} 15^{\prime \prime}$ & 05424000 & 140 & -- & relation & 31.7 & 3.1 \\
\hline 10 & 05424071 & Dead Ck Tr nr Clyman & $43^{\circ} 20^{\prime} 34^{\prime \prime}$ & $88^{\circ} 42^{\prime} 00^{\prime \prime}$ & 05426000 & 5.3 & .0188 & equation & .6 & 1.6 \\
\hline 11 & 05424085 & Wildcat Ck nr Iron Ridge & $43^{\circ} 23^{\prime} 07^{\prime \prime}$ & $88^{\circ} 32^{\prime} 46^{\prime \prime}$ & 05426000 & 21 & -- & relation & 9.1 & 5.9 \\
\hline 12 & 05424093 & Rubicon Ck nr Slinger & $43^{\circ} 19^{\prime} 56^{\prime \prime}$ & $88^{\circ} 18^{\prime} 32^{\prime \prime}$ & 04087000 & 5.24 & .0800 & relation & 2.0 & 5.2 \\
\hline 13 & 05424097 & Rubicon R at Hartford & $43^{\circ} 19^{\prime} 12^{\prime \prime}$ & $88^{\circ} 23^{\prime} 15^{\prime \prime}$ & 04087000 & 27.8 & .0820 & relation & 6.0 & 2.9 \\
\hline 14 & 05424100 & Rubicon R nr Hartford & $43^{\circ} 17^{\prime} 36^{\prime \prime}$ & $88^{\circ} 27^{\prime} 32^{\prime \prime}$ & 04086500 & 60 & -- & relation & 16.1 & 3.6 \\
\hline 15 & 05424150 & Rubicon R nr Hustisford & $43^{\circ} 19^{\prime} 25^{\prime \prime}$ & $88^{\circ} 33^{\prime} 56^{\prime \prime}$ & 05426000 & 80.5 & .0960 & equation & 23.6 & 4.0 \\
\hline 16 & 05424158 & Baker Ck nr Lebanon & $43^{\circ} 15^{\prime} 41^{\prime \prime}$ & $88^{\circ} 36^{\prime} 29^{\prime \prime}$ & 05426000 & 8.63 & .0863 & equation & 2.3 & 3.6 \\
\hline 17 & 05424190 & Ashippun R nr Alderly & $43^{\circ} 14^{\prime} 46^{\prime \prime}$ & $88^{\circ} 25^{\prime} 44^{\prime \prime}$ & 05426000 & 18.6 & .1890 & equation & 7.5 & 5.5 \\
\hline 18 & 05424200 & Ashippun R nr Oconomowoc & $43^{\circ} 10^{\prime} 21^{\prime \prime}$ & $88^{\circ} 27^{\prime} 32^{\prime \prime}$ & 05426000 & 33.8 & .3330 & relation & 12.8 & 5.1 \\
\hline 19 & 05424250 & Ashippun R nr Monterey & $43^{\circ} 10^{\prime} 20^{\prime \prime}$ & $88^{\circ} 32^{\prime} 48^{\prime \prime}$ & 05426000 & 44.6 & .1781 & equation & 17.8 & 5.4 \\
\hline 20 & 05424270 & Rock R Tr nr Ixonia & $43^{\circ} 09^{\prime} 52^{\prime \prime}$ & $88^{\circ} 35^{\prime} 58^{\prime \prime}$ & 05426000 & 3.74 & .0077 & equation & .3 & 1.0 \\
\hline 21 & 05425300 & Oconomowoc R nr Pipersville & $43^{\circ} 06^{\prime} 30^{\prime \prime}$ & $88^{\circ} 37^{\prime} 05^{\prime \prime}$ & 05426000 & 123 & .1433 & equation & 44.7 & 4.9 \\
\hline 22 & 05425320 & Rock R Tr nr Pipersville & $43^{\circ} 07^{\prime} 21^{\prime \prime}$ & $88^{\circ} 39^{\prime} 54^{\prime \prime}$ & 05426000 & 5.31 & .0229 & equation & .7 & 1.8 \\
\hline 23 & 05425340 & Rock R Tr nr Watertown & $43^{\circ} 08^{\prime} 51^{\prime \prime}$ & $88^{\circ} 40^{\prime} 20^{\prime \prime}$ & 05426000 & 8.39 & .0365 & equation & 1.4 & 2.3 \\
\hline 24 & 05425392 & Clyman Ck nr Clyman & $43^{\circ} 16^{\prime} 11^{\prime \prime}$ & $88^{\circ} 43^{\prime} 28^{\prime \prime}$ & 05426000 & 2.38 & .0592 & equation & .5 & 2.9 \\
\hline
\end{tabular}

1 Low-flow discharge measurements at partial-record stations were compared to one or more nearby USGS gaging stations to establish a relation between measured discharge and concurrent daily discharge.

The nearby gaging station that provided the best relation with the partial-record station was chosen as the index station and was associated with nearby miscellaneous measurement sites

${ }^{2}$ Mismatch between surface-water and ground-water drainage area. 
Appendix 12D. Average annual base flow and corresponding recharge, 1970-99, for partial-record stations in the Rock-Fox River Basin-Continued.

[USGS, U.S. Geological Survey; ID, identification number; $\mathrm{dd}^{\circ} \mathrm{mm}$ 'ss", degrees, minutes, seconds; $\mathrm{mi}^{2}$, square mile; $\mathrm{ft}^{3} / \mathrm{s} / \mathrm{mi}^{2}$, cubic foot per second per square mile; $\mathrm{ft}^{3} / \mathrm{s}$, cubic foot per second; equation, statewide regression equation; relation, established relation line with index station; R, River; Bk, Brook; Br, Branch; Fk, Fork; Ck, Creek; Tr, Tributary; nr, near; N, North; S, South; E, East; W, West; --, not determined in historical low-flow reports. See references for full list of low-flow reports.]

\begin{tabular}{|c|c|c|c|c|c|c|c|c|c|c|}
\hline $\begin{array}{c}\text { Map } \\
\text { number }\end{array}$ & $\begin{array}{c}\text { USGS } \\
\text { station ID }\end{array}$ & Station name & $\begin{array}{c}\text { Latitude } \\
\text { (dd }{ }^{\circ} \mathrm{mm} \text { 'ss") }\end{array}$ & $\begin{array}{c}\text { Longitude } \\
\text { (ddºmm'ss") }\end{array}$ & $\begin{array}{l}\text { Index } \\
\text { station }{ }^{1}\end{array}$ & $\begin{array}{c}\text { Drainage } \\
\text { area } \\
\left(\mathrm{mi}^{2}\right)\end{array}$ & $\begin{array}{l}\text { Base flow } \\
\text { factor } \\
\left(\mathrm{ft}^{3} / \mathrm{s} / \mathrm{mi}^{2}\right)\end{array}$ & $\begin{array}{c}\text { Base flow } \\
\text { method }\end{array}$ & $\begin{array}{c}\text { Base flow } \\
\qquad\left(\mathrm{ft}^{3} / \mathrm{s}\right)\end{array}$ & $\begin{array}{c}\text { Recharge } \\
\text { (inches) }\end{array}$ \\
\hline 25 & 05425400 & Silver Ck nr Watertown & $43^{\circ} 13^{\prime} 35^{\prime \prime}$ & $88^{\circ} 43^{\prime} 54^{\prime \prime}$ & 05426000 & 21.9 & 0.0870 & relation & 3.2 & 2.0 \\
\hline 26 & 05425520 & Rock R Tr nr Milford & $43^{\circ} 09^{\prime} 11^{\prime \prime}$ & $88^{\circ} 47^{\prime} 04^{\prime \prime}$ & 05426000 & 9.88 & .1426 & equation & 3.4 & 4.7 \\
\hline 27 & 05425560 & Johnson Ck at Johnson Ck & $43^{\circ} 04^{\prime} 34^{\prime \prime}$ & $88^{\circ} 46^{\prime} 33^{\prime \prime}$ & 05426000 & 43.9 & .0130 & equation & 14.2 & 4.4 \\
\hline 28 & 05425580 & Crawfish R nr Fall R, X & $43^{\circ} 21^{\prime} 52^{\prime \prime}$ & $89^{\circ} 02^{\prime} 55^{\prime \prime}$ & 05426000 & 54.2 & -- & relation & 49.3 & 12.4 \\
\hline 29 & 05425600 & N Br Crawfish R nr Columbus & $43^{\circ} 25^{\prime} 13^{\prime \prime}$ & $89^{\circ} 02^{\prime} 07^{\prime \prime}$ & 05426000 & 69.1 & -- & relation & 5.2 & 1.0 \\
\hline 30 & 05425650 & N Br Crawfish R nr Fall R & $43^{\circ} 22^{\prime} 17^{\prime \prime}$ & $89^{\circ} 02^{\prime} 54^{\prime \prime}$ & 05426000 & 80 & .0151 & equation & 9.0 & 1.5 \\
\hline 31 & 05425700 & Robbins Ck nr Columbus & $43^{\circ} 20^{\prime} 48^{\prime \prime}$ & $89^{\circ} 01^{\prime} 55^{\prime \prime}$ & 05426000 & 8.01 & .0920 & relation & 3.1 & 5.3 \\
\hline 32 & 05425716 & Crawfish $\mathrm{R}$ at Columbus & $43^{\circ} 20^{\prime} 09^{\prime \prime}$ & $89^{\circ} 00^{\prime} 34^{\prime \prime}$ & 05426000 & 164 & -- & relation & 83.2 & 6.9 \\
\hline 33 & 05425830 & Maunesha R nr Sun Prairie & $43^{\circ} 13^{\prime} 11^{\prime \prime}$ & $89^{\circ} 08^{\prime} 06^{\prime \prime}$ & 05426000 & 34.45 & -- & relation & 17.5 & 6.9 \\
\hline 34 & 05425840 & Maunesha R at Marshall & $43^{\circ} 09^{\prime} 57^{\prime \prime}$ & $89^{\circ} 03^{\prime} 28^{\prime \prime}$ & 05426000 & 70.6 & .1060 & relation & 28.9 & 5.6 \\
\hline 35 & 05425847 & Maunesha $\mathrm{R}$ at Waterloo & $43^{\circ} 11^{\prime} 18^{\prime \prime}$ & $88^{\circ} 59^{\prime} 09^{\prime \prime}$ & 05426000 & 91.3 & -- & relation & 40.9 & 6.1 \\
\hline 36 & 05425860 & Stony Bk nr Waterloo & $43^{\circ} 09^{\prime} 30^{\prime \prime}$ & $88^{\circ} 58^{\prime} 56^{\prime \prime}$ & 05426000 & 14.2 & .1463 & equation & 5.0 & 4.8 \\
\hline 37 & 05425898 & Beaver Ck Tr nr Randolph & $43^{\circ} 30^{\prime} 19^{\prime \prime}$ & $89^{\circ} 01^{\prime} 24^{\prime \prime}$ & 05423500 & 4.58 & -- & relation & 1.2 & 3.6 \\
\hline 38 & 05425900 & Beaver Ck nr Randolph & $43^{\circ} 31^{\prime} 00^{\prime \prime}$ & $88^{\circ} 58^{\prime} 23^{\prime \prime}$ & 05423500 & 34.3 & .0431 & equation & 6.5 & 2.6 \\
\hline 39 & 05425926 & Beaverdam R Tr at Reeseville & $43^{\circ} 18^{\prime} 13^{\prime \prime}$ & $88^{\circ} 50^{\prime} 28^{\prime \prime}$ & 05426000 & 3.51 & .0216 & equation & .4 & 1.7 \\
\hline 40 & 05425960 & Crawfish R Tr nr Lk Mills & $43^{\circ} 07^{\prime} 02^{\prime \prime}$ & $88^{\circ} 53^{\prime} 10^{\prime \prime}$ & 05426000 & 5.11 & .1384 & equation & 1.7 & 4.6 \\
\hline 41 & 05425983 & Rock Ck at Lk Mills & $43^{\circ} 04^{\prime} 43^{\prime \prime}$ & $88^{\circ} 54^{\prime} 48^{\prime \prime}$ & 05426000 & 15 & .0397 & equation & 2.7 & 2.4 \\
\hline 42 & 05425988 & Rock Ck at Lk Mills & $43^{\circ} 04^{\prime} 43^{\prime \prime}$ & $88^{\circ} 54^{\prime} 06^{\prime \prime}$ & 05426000 & 15.7 & -- & relation & 2.0 & 1.7 \\
\hline 43 & 05426045 & Deer Ck nr Fort atkinson & $42^{\circ} 57^{\prime} 05^{\prime \prime}$ & $88^{\circ} 48^{\prime} 34^{\prime \prime}$ & 05426000 & 23.9 & .1882 & equation & 9.7 & 5.5 \\
\hline 44 & 05426060 & Bark R at Hartland & $43^{\circ} 07^{\prime} 11^{\prime \prime}$ & $88^{\circ} 20^{\prime} 07^{\prime \prime}$ & 05426000 & 30.2 & .4340 & relation & 16.0 & 7.2 \\
\hline 45 & 05426200 & Bark R nr Sullivan & $42^{\circ} 59^{\prime} 03^{\prime \prime}$ & $88^{\circ} 34^{\prime} 30^{\prime \prime}$ & 05426000 & 104.7 & .2570 & relation & 54.0 & 7.0 \\
\hline 46 & 05426230 & Bark R at Rome & $42^{\circ} 59^{\prime} 10^{\prime \prime}$ & $88^{\circ} 36^{\prime} 58^{\prime \prime}$ & 05426000 & 114 & .3313 & equation & 64.0 & 7.6 \\
\hline 47 & 05426260 & Duck Cr nr Sullivan & $43^{\circ} 02^{\prime} 28^{\prime \prime}$ & $88^{\circ} 35^{\prime} 07^{\prime \prime}$ & 05426000 & 7.44 & -- & relation & 1.3 & 2.4 \\
\hline 48 & 05426300 & Duck Ck nr Sullivan & $42^{\circ} 57^{\prime} 41^{\prime \prime}$ & $88^{\circ} 40^{\prime} 41^{\prime \prime}$ & 05426000 & 33.1 & .1150 & relation & 5.8 & 2.4 \\
\hline
\end{tabular}

${ }^{1}$ Low-flow discharge measurements at partial-record stations were compared to one or more nearby USGS gaging stations to establish a relation between measured discharge and concurrent daily discharge. The nearby gaging station that provided the best relation with the partial-record station was chosen as the index station and was associated with nearby miscellaneous measurement sites.

${ }^{2}$ Mismatch between surface-water and ground-water drainage area. 
[USGS, U.S. Geological Survey; ID, identification number; $\mathrm{dd}^{\circ} \mathrm{mm}$ 'ss", degrees, minutes, seconds; $\mathrm{mi}^{2}$, square mile; $\mathrm{ft}^{3} / \mathrm{s} / \mathrm{mi}^{2}$, cubic foot per second per square mile; $\mathrm{ft}^{3} / \mathrm{s}$, cubic foot per second; equation, statewide regression equation; relation, established relation line with index station; R, River; Bk, Brook; Br, Branch; Fk, Fork; Ck, Creek; Tr, Tributary; nr, near; N, North; S, South; E, East; W, West; ,-- not determined in historical low-flow reports. See references for full list of low-flow reports.]

\begin{tabular}{|c|c|c|c|c|c|c|c|c|c|c|}
\hline $\begin{array}{c}\text { Map } \\
\text { number }\end{array}$ & $\begin{array}{c}\text { USGS } \\
\text { station ID }\end{array}$ & Station name & $\begin{array}{c}\text { Latitude } \\
\text { (dd }{ }^{\circ} \mathrm{mm} \text { 'ss") }\end{array}$ & $\begin{array}{l}\text { Longitude } \\
\text { (dd }{ }^{\circ} \mathrm{mm} \text { 'ss") }\end{array}$ & $\begin{array}{l}\text { Index } \\
\text { station }^{1}\end{array}$ & $\begin{array}{c}\text { Drainage } \\
\text { area } \\
\left(\mathrm{mi}^{2}\right)\end{array}$ & $\begin{array}{c}\text { Base flow } \\
\text { factor } \\
\left(\mathrm{ft}^{3} / \mathrm{s} / \mathrm{mi}^{2}\right)\end{array}$ & $\begin{array}{l}\text { Base flow } \\
\text { method }\end{array}$ & $\begin{array}{c}\text { Base flow } \\
\quad\left(\mathrm{ft}^{3} / \mathrm{s}\right)\end{array}$ & $\begin{array}{c}\text { Recharge } \\
\text { (inches) }\end{array}$ \\
\hline 49 & 05426330 & Bark R at Hebron & $42^{\circ} 55^{\prime} 38^{\prime \prime}$ & $88^{\circ} 41^{\prime} 07^{\prime \prime}$ & 05426000 & 163 & 0.1159 & equation & 53.4 & 4.4 \\
\hline 50 & 05426400 & Scuppernong R nr Palmyra & $42^{\circ} 53^{\prime} 10^{\prime \prime}$ & $88^{\circ} 32^{\prime} 29^{\prime \prime}$ & 05426000 & 24.8 & .4500 & relation & 13.0 & 7.1 \\
\hline 51 & 05426425 & Scuppernong R above Mud Ck & $42^{\circ} 53^{\prime} 53^{\prime \prime}$ & $88^{\circ} 37^{\prime} 24^{\prime \prime}$ & 05426000 & 35.24 & .2875 & equation & 17.9 & 6.9 \\
\hline 52 & 05426430 & Scuppernong R Below Mud Ck & $42^{\circ} 54^{\prime} 02^{\prime \prime}$ & $88^{\circ} 38^{\prime} 13^{\prime \prime}$ & 05426000 & 50.4 & .2319 & equation & 23.1 & 6.2 \\
\hline 53 & 05426437 & Spring Ck nr Whitewater & $42^{\circ} 52^{\prime} 26^{\prime \prime}$ & $88^{\circ} 38^{\prime} 23^{\prime \prime}$ & 05426000 & 10.1 & 1.1277 & equation & 10.2 & 13.7 \\
\hline 54 & 05426450 & Steel Bk Tr nr Whitewater & $42^{\circ} 49^{\prime} 48^{\prime \prime}$ & $88^{\circ} 40^{\prime} 23^{\prime \prime}$ & 05426000 & 3.19 & .4692 & equation & 2.0 & 8.5 \\
\hline 55 & 05426460 & Bark R nr Hebron & $42^{\circ} 53^{\prime} 40^{\prime \prime}$ & $88^{\circ} 42^{\prime} 06^{\prime \prime}$ & 05426000 & 254 & .1608 & equation & 99.5 & 5.3 \\
\hline 56 & 05426900 & Whitewater Ck nr Whitewater & $42^{\circ} 48^{\prime} 14^{\prime \prime}$ & $88^{\circ} 42^{\prime} 11^{\prime \prime}$ & 05431486 & 20.6 & -- & relation & 17.3 & 11.4 \\
\hline 57 & 05427008 & Spring Bk nr Whitewater & $42^{\circ} 47^{\prime} 29^{\prime \prime}$ & $88^{\circ} 45^{\prime} 28^{\prime \prime}$ & 05431486 & 11.05 & .2473 & equation & 5.1 & 6.3 \\
\hline 58 & 05427010 & Spring Bk at Whitewater & $42^{\circ} 49^{\prime} 08^{\prime \prime}$ & $88^{\circ} 44^{\prime} 41^{\prime \prime}$ & 05431486 & 16.28 & -- & relation & 7.1 & 5.9 \\
\hline 59 & 05427013 & Whitewater $\mathrm{Ck}$ at Whitewater & $42^{\circ} 50^{\prime} 34^{\prime \prime}$ & $88^{\circ} 44^{\prime} 02^{\prime \prime}$ & 05431486 & 42.7 & .3170 & relation & 23.5 & 7.5 \\
\hline 60 & 05427014 & Whitewater Ck Tr nr Whitewater & $42^{\circ} 51^{\prime} 18^{\prime \prime}$ & $88^{\circ} 43^{\prime} 08^{\prime \prime}$ & 05431486 & 3.95 & -- & relation & .8 & 2.8 \\
\hline 61 & 05427017 & Whitewater Ck nr Whitewater & $42^{\circ} 51^{\prime} 26^{\prime \prime}$ & $88^{\circ} 44^{\prime} 26^{\prime \prime}$ & 05431486 & 49.39 & .3061 & equation & 26.2 & 7.2 \\
\hline 62 & 05427037 & Whitewater Ck at Cold Spring & $42^{\circ} 53^{\prime} 33^{\prime \prime}$ & $88^{\circ} 46^{\prime} 22^{\prime \prime}$ & 05431486 & 70 & -- & relation & 31.6 & 6.1 \\
\hline 63 & 05427130 & Rock R Tr nr Busseyville & $42^{\circ} 56^{\prime} 05^{\prime \prime}$ & $88^{\circ} 54^{\prime} 28^{\prime \prime}$ & 05426000 & 8.73 & .0922 & equation & 2.4 & 3.7 \\
\hline 64 & 05427200 & Allen Ck nr Fort atkinson & $42^{\circ} 53^{\prime} 54 "$ & $88^{\circ} 51^{\prime} 35^{\prime \prime}$ & 05431486 & 10.2 & .0700 & relation & 4.2 & 5.6 \\
\hline 65 & 05427223 & Rock R Tr nr Busseyville & $42^{\circ} 55^{\prime} 27^{\prime \prime}$ & $88^{\circ} 57^{\prime} 07^{\prime \prime}$ & 05426000 & 9.83 & .1158 & equation & 3.0 & 4.2 \\
\hline 66 & 05427230 & Otter Ck nr Milton & $42^{\circ} 49^{\prime} 19^{\prime \prime}$ & $88^{\circ} 55^{\prime} 52^{\prime \prime}$ & 05426000 & 47.4 & .0360 & relation & 3.6 & 1.0 \\
\hline 67 & 05427350 & Koshkonong Cr nr Deerfield & $43^{\circ} 04^{\prime} 25^{\prime \prime}$ & $89^{\circ} 04^{\prime} 25^{\prime \prime}$ & 05426000 & 68.6 & .3440 & relation & 21.2 & 4.2 \\
\hline 68 & 05427430 & Koshkonong $\mathrm{Ck}$ at London & $43^{\circ} 03^{\prime} 31^{\prime \prime}$ & $89^{\circ} 00^{\prime} 35^{\prime \prime}$ & 05426000 & 108 & .2369 & equation & 50.9 & 6.4 \\
\hline 69 & 05427510 & Koshkonong Ck nr Busseyville & $42^{\circ} 54^{\prime} 44^{\prime \prime}$ & $89^{\circ} 00^{\prime} 15^{\prime \prime}$ & 05426000 & 161 & .2348 & equation & 76.1 & 6.4 \\
\hline 70 & 05427550 & Saunders Ck at Albion & $42^{\circ} 52^{\prime} 43^{\prime \prime}$ & $89^{\circ} 03^{\prime} 57^{\prime \prime}$ & 05431486 & 21.2 & .2430 & relation & 12.2 & 7.8 \\
\hline 71 & 05427640 & Yahara R at Deforest & $43^{\circ} 14^{\prime} 58^{\prime \prime}$ & $89^{\circ} 20^{\prime} 37^{\prime \prime}$ & 05406500 & 39.2 & -- & relation & 7.2 & ${ }^{2} 2.5$ \\
\hline 72 & 05427700 & Yahara R nr Deforest & $43^{\circ} 12^{\prime} 59^{\prime \prime}$ & $89^{\circ} 20^{\prime} 58^{\prime \prime}$ & 05406500 & 48.6 & .1010 & relation & 8.5 & 22.4 \\
\hline
\end{tabular}

${ }^{1}$ Low-flow discharge measurements at partial-record stations were compared to one or more nearby USGS gaging stations to establish a relation between measured discharge and concurrent daily discharge. The nearby gaging station that provided the best relation with the partial-record station was chosen as the index station and was associated with nearby miscellaneous measurement sites.

${ }^{2}$ Mismatch between surface-water and ground-water drainage area. 
Appendix 12D. Average annual base flow and corresponding recharge, 1970-99, for partial-record stations in the Rock-Fox River Basin—Continued.

[USGS, U.S. Geological Survey; ID, identification number; $\mathrm{dd}^{\circ} \mathrm{mm}$ 'ss", degrees, minutes, seconds; $\mathrm{mi}^{2}$, square mile; $\mathrm{ft}^{3} / \mathrm{s} / \mathrm{mi}^{2}$, cubic foot per second per square mile; $\mathrm{ft}^{3} / \mathrm{s}$, cubic foot per second; equation, statewide regression equation; relation, established relation line with index station; R, River; Bk, Brook; Br, Branch; Fk, Fork; Ck, Creek; Tr, Tributary; nr, near; N, North; S, South; E, East; W, West; ,-- not determined in historical low-flow reports. See references for full list of low-flow reports.]

\begin{tabular}{|c|c|c|c|c|c|c|c|c|c|c|}
\hline $\begin{array}{c}\text { Map } \\
\text { number }\end{array}$ & $\begin{array}{c}\text { USGS } \\
\text { station ID }\end{array}$ & Station name & $\begin{array}{c}\text { Latitude } \\
\text { (dd }{ }^{\circ} \mathrm{mm} \text { 'ss") }\end{array}$ & $\begin{array}{l}\text { Longitude } \\
\text { (ddºmm'ss") }\end{array}$ & $\begin{array}{l}\text { Index } \\
\text { station }^{1}\end{array}$ & $\begin{array}{c}\text { Drainage } \\
\text { area } \\
\left(\mathrm{mi}^{2}\right)\end{array}$ & $\begin{array}{c}\text { Base flow } \\
\text { factor } \\
\left(\mathrm{ft}^{3} / \mathrm{s} / \mathrm{mi}^{2}\right)\end{array}$ & $\begin{array}{c}\text { Base flow } \\
\text { method }\end{array}$ & $\begin{array}{c}\text { Base flow } \\
\left(\mathrm{ft}^{3} / \mathrm{s}\right)\end{array}$ & $\begin{array}{c}\text { Recharge } \\
\text { (inches) }\end{array}$ \\
\hline 73 & 05427767 & Token Ck nr Token Ck & $43^{\circ} 11 ' 53^{\prime \prime}$ & $89^{\circ} 17^{\prime} 35^{\prime \prime}$ & 05406500 & 4.78 & -- & relation & 9.4 & 226.7 \\
\hline 74 & 05427910 & Sixmile Ck nr Waunakee & $43^{\circ} 08^{\prime} 49^{\prime \prime}$ & $89^{\circ} 26^{\prime} 13^{\prime \prime}$ & 05406500 & 48.3 & 0.0987 & equation & 14.2 & 4.0 \\
\hline 75 & 05427927 & Spring Ck nr Waunakee & $43^{\circ} 08^{\prime} 34^{\prime \prime}$ & $89^{\circ} 27^{\prime} 56^{\prime \prime}$ & 05406500 & 9.95 & -- & relation & 2.3 & 3.1 \\
\hline 76 & 05427930 & Spring Ck nr Waunakee & $43^{\circ} 08^{\prime} 25^{\prime \prime}$ & $89^{\circ} 26^{\prime} 32^{\prime \prime}$ & 05406500 & 12.6 & -- & relation & 4.6 & 5.0 \\
\hline 77 & 05427952 & Pheasant $\mathrm{Br}$ at Middleton & $43^{\circ} 06^{\prime} 22^{\prime \prime}$ & $89^{\circ} 29^{\prime} 01^{\prime \prime}$ & 05406500 & 24.5 & -- & relation & 5.8 & 3.2 \\
\hline 78 & 05428600 & W Br Starkweather Ck at Madison & $43^{\circ} 06^{\prime} 00^{\prime \prime}$ & $89^{\circ} 20^{\prime} 18^{\prime \prime}$ & 05426000 & 10.08 & -- & relation & 3.8 & 5.1 \\
\hline 79 & 05428650 & E Br Starkweather Ck at Madison & $43^{\circ} 05^{\prime} 58^{\prime \prime}$ & $89^{\circ} 19^{\prime} 54^{\prime \prime}$ & 05426000 & 7.41 & -- & relation & 3.5 & 6.4 \\
\hline 80 & 05429570 & Door $\mathrm{Ck}$ Tr at Cottage Grove & $43^{\circ} 04^{\prime} 19^{\prime \prime}$ & $89^{\circ} 12^{\prime} 52^{\prime \prime}$ & 05426000 & 2.73 & -- & relation & 1.5 & 7.5 \\
\hline 81 & 05429580 & Door Ck nr Cotage Grove & $43^{\circ} 02^{\prime} 54^{\prime \prime}$ & $89^{\circ} 13^{\prime} 54^{\prime \prime}$ & 05426000 & 12.24 & -- & relation & 5.4 & 6.0 \\
\hline 82 & 05429600 & Door Ck nr Mcfarland & $43^{\circ} 00^{\prime} 46^{\prime \prime}$ & $89^{\circ} 14^{\prime} 08^{\prime \prime}$ & 05426000 & 26.7 & .1240 & relation & 12.0 & 6.1 \\
\hline 83 & 05430040 & Oregon $\mathrm{Br}$ nr Oregon & $42^{\circ} 55^{\prime} 24^{\prime \prime}$ & $89^{\circ} 21^{\prime 22 "}$ & 05436500 & 21.4 & .0609 & equation & 4.8 & 3.1 \\
\hline 84 & 05430055 & Oregon Br nr Oregon & $42^{\circ} 55^{\prime} 10^{\prime \prime}$ & $89^{\circ} 18^{\prime} 50^{\prime \prime}$ & 05436500 & 27.9 & .0974 & equation & 8.1 & 3.9 \\
\hline 85 & 05430085 & Rutland Br Badfish Ck nr Bklyn & $42^{\circ} 53^{\prime} 45^{\prime \prime}$ & $89^{\circ} 19^{\prime} 15^{\prime \prime}$ & 05436500 & 5.94 & 1.0575 & equation & 5.7 & 13.1 \\
\hline 86 & 05430300 & Marsh Ck nr Janesville & $42^{\circ} 43^{\prime} 15^{\prime \prime}$ & $89^{\circ} 03^{\prime} 30^{\prime \prime}$ & 05436500 & 35.8 & .4320 & relation & 18.0 & 6.8 \\
\hline 87 & 05430400 & Fisher Ck nr Janesville & $42^{\circ} 39^{\prime} 44^{\prime \prime}$ & $89^{\circ} 03^{\prime} 31^{\prime \prime}$ & 05436500 & 3.93 & .0453 & equation & .7 & 2.5 \\
\hline 88 & 05430450 & Markham Ck nr Janesville & $42^{\circ} 38^{\prime} 48^{\prime \prime}$ & $89^{\circ} 04^{\prime} 00^{\prime \prime}$ & 05436500 & 9.99 & .0792 & equation & 2.5 & 3.5 \\
\hline 89 & 05430525 & Bass $\mathrm{Ck}$ at Footville & $42^{\circ} 39^{\prime} 45^{\prime \prime}$ & $89^{\circ} 12^{\prime} 32^{\prime \prime}$ & 05436500 & 14 & -- & relation & 7.4 & 7.2 \\
\hline 90 & 05430530 & Stevens Ck nr Footville & $42^{\circ} 41^{\prime} 16^{\prime \prime}$ & $89^{\circ} 10^{\prime} 31^{\prime \prime}$ & 05436500 & 7.22 & .0741 & equation & 1.8 & 3.3 \\
\hline 91 & 05430535 & Stevens Ck Tr nr Footville & $42^{\circ} 41^{\prime} 13^{\prime \prime}$ & $89^{\circ} 10^{\prime} 13^{\prime \prime}$ & 05436500 & 3.18 & .1095 & equation & .9 & 4.0 \\
\hline 92 & 05430560 & Bass $\mathrm{Ck}$ Tr at Hanover & $42^{\circ} 38^{\prime} 36^{\prime \prime}$ & $89^{\circ} 10^{\prime} 29^{\prime \prime}$ & 05436500 & 14.7 & .1069 & equation & 4.4 & 4.1 \\
\hline 93 & 05430580 & Bass Ck at Hanover & $42^{\circ} 38^{\prime} 31^{\prime \prime}$ & $89^{\circ} 09^{\prime} 44^{\prime \prime}$ & 05436500 & 47 & .2165 & equation & 20.8 & 6.0 \\
\hline 94 & 05430600 & Bass Ck nr Janesville & $42^{\circ} 37^{\prime} 20^{\prime \prime}$ & $89^{\circ} 07^{\prime} 45^{\prime \prime}$ & 05436500 & 57.9 & .2920 & relation & 26.9 & 6.3 \\
\hline 95 & 05430610 & Bass Ck at Afton & $42^{\circ} 36^{\prime} 04^{\prime \prime}$ & $89^{\circ} 03^{\prime} 36^{\prime \prime}$ & 05436500 & 65.5 & .2483 & equation & 31.3 & 6.5 \\
\hline 96 & 05430650 & Rock R Tr nr Beloit & $42^{\circ} 33^{\prime} 53^{\prime \prime}$ & $89^{\circ} 03^{\prime} 04^{\prime \prime}$ & 05436500 & 12.3 & .0806 & equation & 3.2 & 3.5 \\
\hline
\end{tabular}

${ }^{1}$ Low-flow discharge measurements at partial-record stations were compared to one or more nearby USGS gaging stations to establish a relation between measured discharge and concurrent daily discharge. The nearby gaging station that provided the best relation with the partial-record station was chosen as the index station and was associated with nearby miscellaneous measurement sites.

${ }^{2}$ Mismatch between surface-water and ground-water drainage area. 
[USGS, U.S. Geological Survey; ID, identification number; $\mathrm{dd}^{\circ} \mathrm{mm}$ 'ss", degrees, minutes, seconds; $\mathrm{mi}^{2}$, square mile; $\mathrm{ft}^{3} / \mathrm{s} / \mathrm{mi}^{2}$, cubic foot per second per square mile; $\mathrm{ft}^{3} / \mathrm{s}$, cubic foot per second; equation, statewide regression equation; relation, established relation line with index station; R, River; Bk, Brook; Br, Branch; Fk, Fork; Ck, Creek; Tr, Tributary; nr, near; N, North; S, South; E, East; W, West;

--, not determined in historical low-flow reports. See references for full list of low-flow reports.]

\begin{tabular}{|c|c|c|c|c|c|c|c|c|c|c|}
\hline $\begin{array}{c}\text { Map } \\
\text { number }\end{array}$ & $\begin{array}{c}\text { USGS } \\
\text { station ID }\end{array}$ & Station name & $\begin{array}{c}\text { Latitude } \\
\text { (dd }{ }^{\circ} \mathrm{mm} \text { 'ss") }\end{array}$ & $\begin{array}{l}\text { Longitude } \\
\text { (dd }{ }^{\circ} \mathrm{mm} \text { 'ss") }\end{array}$ & $\begin{array}{l}\text { Index } \\
\text { station }\end{array}$ & $\begin{array}{c}\text { Drainage } \\
\text { area } \\
\left(\mathrm{mi}^{2}\right)\end{array}$ & $\begin{array}{c}\text { Base flow } \\
\text { factor } \\
\left(\mathrm{ft}^{3} / \mathrm{s} / \mathrm{mi}^{2}\right)\end{array}$ & $\begin{array}{l}\text { Base flow } \\
\text { method }\end{array}$ & $\begin{array}{c}\text { Base flow } \\
\qquad\left(\mathrm{ft}^{3} / \mathrm{s}\right)\end{array}$ & $\begin{array}{c}\text { Recharge } \\
\text { (inches) }\end{array}$ \\
\hline 97 & 05431005 & Turtle Ck nr Delavan & $42^{\circ} 40^{\prime} 59^{\prime \prime}$ & $88^{\circ} 41^{\prime} 16^{\prime \prime}$ & 05431486 & 14.2 & -- & relation & 7.3 & 7.0 \\
\hline 98 & 05431010 & Turtle Ck Tr nr Delavan & $42^{\circ} 39^{\prime} 27^{\prime \prime}$ & $88^{\circ} 37^{\prime} 42^{\prime \prime}$ & 05431486 & 6.59 & 0.0286 & equation & 1.0 & 2.0 \\
\hline 99 & 05431015 & Jackson Ck nr Elkhorn & $42^{\circ} 38^{\prime} 45^{\prime \prime}$ & $88^{\circ} 32^{\prime} 29^{\prime \prime}$ & 05431486 & 9.69 & -- & relation & 1.4 & 2.0 \\
\hline 100 & 05431016 & $\begin{array}{l}\text { Johnson Ck at Mounds Rd nr } \\
\text { Elkhorn }\end{array}$ & $42^{\circ} 38^{\prime} 27^{\prime \prime}$ & $88^{\circ} 33^{\prime} 39^{\prime \prime}$ & 05431486 & 16.8 & .1041 & equation & 5.0 & 4.0 \\
\hline 101 & 05431070 & Turtle Ck nr Allens Grove & $42^{\circ} 37^{\prime} 51^{\prime \prime}$ & $88^{\circ} 46^{\prime} 30^{\prime \prime}$ & 05431486 & 111 & .2848 & equation & 57.6 & 7.0 \\
\hline 102 & 05431200 & Little Turtle Ck nr Allens Grove & $42^{\circ} 32^{\prime} 12^{\prime \prime}$ & $88^{\circ} 48^{\prime} 04^{\prime \prime}$ & 05431486 & 18.2 & .3160 & equation & 9.6 & 7.2 \\
\hline 103 & 05431250 & Ladd Ck nr Allens Grove & $42^{\circ} 32^{\prime} 20^{\prime \prime}$ & $88^{\circ} 44^{\prime} 50^{\prime \prime}$ & 05431486 & 6.35 & -- & relation & 2.0 & 4.3 \\
\hline 104 & 05431300 & Ladd Ck nr Allens Grove & $42^{\circ} 33^{\prime} 14^{\prime \prime}$ & $88^{\circ} 46^{\prime} 12^{\prime \prime}$ & 05431486 & 12.6 & .1895 & equation & 5.1 & 5.5 \\
\hline 105 & 05431350 & Little Turtle Ck Tr nr Allens Grove & $42^{\circ} 33^{\prime} 53^{\prime \prime}$ & $88^{\circ} 46^{\prime} 35^{\prime \prime}$ & 05431486 & 6.46 & .1707 & equation & 2.4 & 5.1 \\
\hline 106 & 05431400 & Little Turtle $\mathrm{Ck}$ at Allens Grove & $42^{\circ} 34^{\prime} 33^{\prime \prime}$ & $88^{\circ} 45^{\prime} 40^{\prime \prime}$ & 05431486 & 42.4 & .0810 & relation & 19.1 & 6.1 \\
\hline 107 & 05431420 & Little Turtle Ck Tr nr Allens Grove & $42^{\circ} 35^{\prime} 06^{\prime \prime}$ & $88^{\circ} 44^{\prime} 28^{\prime \prime}$ & 05431486 & 11.2 & -- & relation & 3.1 & 3.8 \\
\hline 108 & 05431480 & Spring Bk nr Clinton & $42^{\circ} 36^{\prime} 04^{\prime \prime}$ & $88^{\circ} 49^{\prime} 44^{\prime \prime}$ & 05431486 & 10 & .0523 & equation & 2.0 & 2.8 \\
\hline 109 & 05431600 & Turtle Ck Tr \# 4 at Shopiere & $42^{\circ} 34^{\prime} 38^{\prime \prime}$ & $88^{\circ} 55^{\prime} 24^{\prime \prime}$ & 05431486 & 3.32 & .1198 & equation & 1.0 & 4.2 \\
\hline 110 & 05431740 & Spring Bk nr Beloit & $42^{\circ} 30^{\prime} 37^{\prime \prime}$ & $88^{\circ} 59^{\prime} 05^{\prime \prime}$ & 05431486 & 9.33 & .1618 & equation & 3.4 & 5.0 \\
\hline 111 & 05437180 & E Fk Raccoon Ck nr Beloit & $42^{\circ} 30^{\prime} 41^{\prime \prime}$ & $89^{\circ} 07^{\prime} 16^{\prime \prime}$ & 05436500 & 10.6 & .1890 & relation & 6.1 & 7.8 \\
\hline 112 & 05437200 & E Fk Racoon Ck Tr nr Beloit & $42^{\circ} 30^{\prime} 44^{\prime \prime}$ & $89^{\circ} 06^{\prime} 40^{\prime \prime}$ & 05436500 & 4.64 & .1140 & relation & 4.4 & 12.9 \\
\hline 113 & 05437210 & E Fk Raccoon Ck nr Beloit & $42^{\circ} 30^{\prime} 15^{\prime \prime}$ & $89^{\circ} 07^{\prime} 02^{\prime \prime}$ & 05436500 & 16.2 & .2350 & relation & 9.5 & 8.0 \\
\hline 114 & 054382832 & Raccoon Ck nr Sharon & $42^{\circ} 30^{\prime} 33^{\prime \prime}$ & $88^{\circ} 39^{\prime} 47^{\prime \prime}$ & 05431486 & 11.2 & -- & relation & 4.9 & 5.9 \\
\hline 115 & 05527662 & Brighton Ck nr Paddock Lk & $42^{\circ} 34^{\prime} 57^{\prime \prime}$ & $88^{\circ} 04^{\prime} 04^{\prime \prime}$ & 05545750 & 16.6 & .0572 & equation & 3.6 & 2.9 \\
\hline 116 & 05527672 & Salem Br nr Salem & $42^{\circ} 33^{\prime} 48^{\prime \prime}$ & $88^{\circ} 05^{\prime} 29^{\prime \prime}$ & 05545750 & 3.77 & .1237 & equation & 1.2 & 4.3 \\
\hline 117 & 05527675 & Brighton Ck nr Bristol & $42^{\circ} 34^{\prime} 42^{\prime \prime}$ & $88^{\circ} 02^{\prime} 56^{\prime \prime}$ & 05545750 & 25.5 & .0562 & equation & 5.5 & 2.9 \\
\hline 118 & 05527700 & Des Plaines R nr Kenosha & $42^{\circ} 32^{\prime} 37^{\prime \prime}$ & $88^{\circ} 00^{\prime} 04^{\prime \prime}$ & 05545750 & 56.2 & .0210 & equation & 7.4 & 1.8 \\
\hline 119 & 05527720 & Des Plaines R nr Pleasant Priarie & $42^{\circ} 33^{\prime} 14^{\prime \prime}$ & $87^{\circ} 57^{\prime} 08^{\prime \prime}$ & 05545750 & 71.5 & .0331 & equation & 12.0 & 2.3 \\
\hline
\end{tabular}

${ }^{1}$ Low-flow discharge measurements at partial-record stations were compared to one or more nearby USGS gaging stations to establish a relation between measured discharge and concurrent daily discharge. The nearby gaging station that provided the best relation with the partial-record station was chosen as the index station and was associated with nearby miscellaneous measurement sites.

${ }^{2}$ Mismatch between surface-water and ground-water drainage area. 
Appendix 12D. Average annual base flow and corresponding recharge, 1970-99, for partial-record stations in the Rock-Fox River Basin-Continued.

[USGS, U.S. Geological Survey; ID, identification number; $\mathrm{dd}^{\circ} \mathrm{mm}$ 'ss", degrees, minutes, seconds; $\mathrm{mi}^{2}$, square mile; $\mathrm{ft}^{3} / \mathrm{s} / \mathrm{mi}^{2}$, cubic foot per second per square mile; $\mathrm{ft}^{3} / \mathrm{s}$, cubic foot per second; equation, statewide regression equation; relation, established relation line with index station; R, River; Bk, Brook; Br, Branch; Fk, Fork; Ck, Creek; Tr, Tributary; nr, near; N, North; S, South; E, East; W, West; ,-- not determined in historical low-flow reports. See references for full list of low-flow reports.]

\begin{tabular}{|c|c|c|c|c|c|c|c|c|c|c|}
\hline $\begin{array}{c}\text { Map } \\
\text { number }\end{array}$ & $\begin{array}{c}\text { USGS } \\
\text { station ID }\end{array}$ & Station name & $\begin{array}{c}\text { Latitude } \\
\text { (dd }{ }^{\circ} \mathrm{mm} \text { 'ss") }\end{array}$ & $\begin{array}{l}\text { Longitude } \\
\text { (dd }{ }^{\circ} \mathrm{mm} \text { 'ss") }\end{array}$ & $\begin{array}{l}\text { Index } \\
\text { station }{ }^{1}\end{array}$ & $\begin{array}{c}\text { Drainage } \\
\text { area } \\
\left(\mathrm{mi}^{2}\right)\end{array}$ & $\begin{array}{c}\text { Base flow } \\
\text { factor } \\
\left(\mathrm{ft}^{3} / \mathrm{s} / \mathrm{mi}^{2}\right)\end{array}$ & $\begin{array}{l}\text { Base flow } \\
\text { method }\end{array}$ & $\begin{array}{c}\text { Base flow } \\
\quad\left(\mathrm{ft}^{3} / \mathrm{s}\right)\end{array}$ & $\begin{array}{c}\text { Recharge } \\
\text { (inches) }\end{array}$ \\
\hline 120 & 05527895 & Dutch Gap Canal nr Benet Lk & $42^{\circ} 29^{\prime} 43^{\prime \prime}$ & $88^{\circ} 00^{\prime} 18^{\prime \prime}$ & 05545750 & 16.1 & 0.0364 & equation & 2.8 & 2.3 \\
\hline 121 & 055437888 & Fox R Tr nr Sussex & $43^{\circ} 07^{\prime} 17^{\prime \prime}$ & $88^{\circ} 09^{\prime} 54^{\prime \prime}$ & 05543830 & 5.39 & .2276 & equation & 2.3 & 5.9 \\
\hline 122 & 055437901 & Fox R nr Sussex & $43^{\circ} 06^{\prime} 27^{\prime \prime}$ & $88^{\circ} 10^{\prime} 20^{\prime \prime}$ & 05543830 & 22.01 & .0578 & equation & 4.8 & 3.0 \\
\hline 123 & 055437907 & Fox R Tr nr Sussex & $43^{\circ} 05^{\prime} 27^{\prime \prime}$ & $88^{\circ} 12^{\prime} 19^{\prime \prime}$ & 05543830 & 10.5 & .3694 & equation & 5.9 & 7.7 \\
\hline 124 & 05543800 & $\begin{array}{l}\text { Fox R nr Waukesha at Watertown } \\
\text { Rd }\end{array}$ & $43^{\circ} 03^{\prime} 12^{\prime \prime}$ & $88^{\circ} 11^{\prime} 41^{\prime \prime}$ & 05543830 & 77.4 & .1150 & relation & 36.1 & 6.3 \\
\hline 125 & 05543808 & Zion Ck nr Pewaukee & $43^{\circ} 03^{\prime} 27^{\prime \prime}$ & $88^{\circ} 18^{\prime} 50^{\prime \prime}$ & 05545750 & 3.4 & .3046 & equation & 1.7 & 6.8 \\
\hline 126 & 05543850 & Pebble Ck nr Waukesha & $42^{\circ} 59^{\prime} 39^{\prime \prime}$ & $88^{\circ} 17^{\prime} 22^{\prime \prime}$ & 05543830 & 17.3 & .3360 & relation & 9.4 & 7.4 \\
\hline 127 & 05544080 & Jericho Ck nr Jericho & $42^{\circ} 51^{\prime} 32^{\prime \prime}$ & $88^{\circ} 25^{\prime} 57^{\prime \prime}$ & 05545750 & 12.2 & .1625 & equation & 4.5 & 5.0 \\
\hline 128 & 05544320 & Fox $\mathrm{R}$ at Big Bend & $42^{\circ} 52^{\prime} 34^{\prime \prime}$ & $88^{\circ} 12^{\prime} 38^{\prime \prime}$ & 05545750 & 322 & .2410 & relation & 168.0 & 7.1 \\
\hline 129 & 05544350 & Fox R at Waterford & $42^{\circ} 45^{\prime} 46^{\prime \prime}$ & $88^{\circ} 12^{\prime} 48^{\prime \prime}$ & 05545750 & 357 & .2787 & equation & 187.4 & 7.1 \\
\hline 130 & 05544838 & Honey $\mathrm{Ck}$ at $\mathrm{E}$ Troy & $42^{\circ} 463^{\prime} 53^{\prime \prime}$ & $88^{\circ} 25^{\prime} 31^{\prime \prime}$ & 05431486 & 40.9 & .2979 & equation & 21.3 & 7.1 \\
\hline 131 & 05544850 & Honey Ck nr E Troy & $42^{\circ} 47^{\prime} 18^{\prime \prime}$ & $88^{\circ} 22^{\prime} 00^{\prime \prime}$ & 05431486 & 48.4 & -- & relation & 19.8 & 5.6 \\
\hline 132 & 05544970 & Honey $\mathrm{Ck}$ at Honey $\mathrm{Ck}$ & $42^{\circ} 44^{\prime} 54^{\prime \prime}$ & $88^{\circ} 18^{\prime} 36^{\prime \prime}$ & 05431486 & 74.2 & -- & relation & 27.7 & 5.1 \\
\hline 133 & 05544990 & Honey Ck nr Burlington & $42^{\circ} 43^{\prime} 09^{\prime \prime}$ & $88^{\circ} 18^{\prime} 03^{\prime \prime}$ & 05545750 & 84.8 & -- & relation & 29.3 & 4.7 \\
\hline 134 & 05545080 & Sugar Ck nr Elkhorn & $42^{\circ} 42^{\prime} 54^{\prime \prime}$ & $88^{\circ} 33^{\prime} 44^{\prime \prime}$ & 05431486 & 27.2 & -- & relation & 5.0 & 2.5 \\
\hline 135 & 05545109 & Sugar Ck nr Spring Prairie & $42^{\circ} 43^{\prime} 21^{\prime \prime}$ & $88^{\circ} 28^{\prime} 17^{\prime \prime}$ & 05431486 & 54.8 & -- & relation & 13.6 & 3.4 \\
\hline 136 & 05545110 & Suger Ck nr Troy & $42^{\circ} 43^{\prime} 55^{\prime \prime}$ & $88^{\circ} 24^{\prime} 21^{\prime \prime}$ & 05431486 & 60.9 & -- & relation & 16.6 & 3.7 \\
\hline 137 & 05545118 & Sugar Ck Tr nr Honey Lk & $42^{\circ} 43^{\prime} 01^{\prime \prime}$ & $88^{\circ} 21^{\prime} 50^{\prime \prime}$ & 05431486 & 4.95 & -- & relation & 3.4 & 9.3 \\
\hline 138 & 05545120 & Sugar Ck nr Vienna & $42^{\circ} 42^{\prime} 54^{\prime \prime}$ & $88^{\circ} 20^{\prime} 31^{\prime \prime}$ & 05431486 & 76.4 & .2080 & relation & 28.0 & 5.0 \\
\hline 139 & 05545203 & White R Tr at Lyons & $42^{\circ} 39^{\prime} 43^{\prime \prime}$ & $88^{\circ} 21 ' 21 "$ & 05431486 & 4.11 & .1722 & equation & 1.5 & 5.1 \\
\hline 140 & 05545208 & White R Tr nr Lyons & $42^{\circ} 39^{\prime} 40^{\prime \prime}$ & $88^{\circ} 20^{\prime} 15^{\prime \prime}$ & 05431486 & 2.9 & .1298 & equation & .9 & 4.4 \\
\hline 141 & 05545240 & White R Tr nr Pell Lk & $42^{\circ} 35^{\prime} 14^{\prime \prime}$ & $88^{\circ} 20^{\prime} 23^{\prime \prime}$ & 05431486 & 10.5 & .3455 & equation & 5.7 & 7.4 \\
\hline 142 & 05545300 & White $\mathrm{R}$ at Burlington & $42^{\circ} 39^{\prime} 57^{\prime \prime}$ & $88^{\circ} 19^{\prime} 03^{\prime \prime}$ & 05431486 & 110 & .2715 & relation & 46.0 & 5.7 \\
\hline
\end{tabular}

${ }^{1}$ Low-flow discharge measurements at partial-record stations were compared to one or more nearby USGS gaging stations to establish a relation between measured discharge and concurrent daily discharge. The nearby gaging station that provided the best relation with the partial-record station was chosen as the index station and was associated with nearby miscellaneous measurement sites.

${ }^{2}$ Mismatch between surface-water and ground-water drainage area. 
Appendix 12D. Average annual base flow and corresponding recharge, 1970-99, for partial-record stations in the Rock-Fox River Basin-Continued.

[USGS, U.S. Geological Survey; ID, identification number; $\mathrm{dd}^{\circ} \mathrm{mm}$ 'ss", degrees, minutes, seconds; $\mathrm{mi}^{2}$, square mile; $\mathrm{ft}^{3} / \mathrm{s} / \mathrm{mi}^{2}$, cubic foot per second per square mile; $\mathrm{ft}^{3} / \mathrm{s}$, cubic foot per second; equation, statewide regression equation; relation, established relation line with index station; R, River; Bk, Brook; Br, Branch; Fk, Fork; Ck, Creek; Tr, Tributary; nr, near; N, North; S, South; E, East; W, West; ,-- not determined in historical low-flow reports. See references for full list of low-flow reports.]

\begin{tabular}{|c|c|c|c|c|c|c|c|c|c|c|}
\hline $\begin{array}{c}\text { Map } \\
\text { number }\end{array}$ & $\begin{array}{c}\text { USGS } \\
\text { station ID }\end{array}$ & Station name & $\begin{array}{c}\text { Latitude } \\
\text { (dd }{ }^{\circ} \mathrm{mm} \text { 'ss") }\end{array}$ & $\begin{array}{c}\text { Longitude } \\
\text { (dd }{ }^{\circ} \mathrm{mm} \text { 'ss") }\end{array}$ & $\begin{array}{l}\text { Index } \\
\text { station }{ }^{1}\end{array}$ & $\begin{array}{c}\text { Drainage } \\
\text { area } \\
\left(\mathrm{mi}^{2}\right)\end{array}$ & $\begin{array}{c}\text { Base flow } \\
\text { factor } \\
\left(\mathrm{ft}^{3} / \mathrm{s} / \mathrm{mi}^{2}\right)\end{array}$ & $\begin{array}{l}\text { Base flow } \\
\text { method }\end{array}$ & $\begin{array}{c}\text { Base flow } \\
\left(\mathrm{ft}^{3} / \mathrm{s}\right)\end{array}$ & $\begin{array}{c}\text { Recharge } \\
\text { (inches) }\end{array}$ \\
\hline 143 & 05545329 & Honey $\mathrm{Ck}$ at Burlington & $42^{\circ} 40^{\prime} 53^{\prime \prime}$ & $88^{\circ} 16^{\prime} 34^{\prime \prime}$ & 05545750 & 288 & 0.1888 & equation & 123.0 & 5.8 \\
\hline 144 & 05545600 & Hoosier Ck nr Burlington & $42^{\circ} 39^{\prime} 30^{\prime \prime}$ & $88^{\circ} 14^{\prime} 01^{\prime \prime}$ & 05545750 & 20.7 & .1250 & relation & 6.6 & 4.3 \\
\hline 145 & 05545955 & Basset Ck nr Twin Lks & $42^{\circ} 32^{\prime} 27^{\prime \prime}$ & $88^{\circ} 13^{\prime} 40^{\prime \prime}$ & 05545750 & 5.09 & .2007 & equation & 2.1 & 5.5 \\
\hline 146 & 05548150 & N Br Nippersink Ck nr Genoa City & $42^{\circ} 30^{\prime} 15^{\prime \prime}$ & $88^{\circ} 23^{\prime} 01^{\prime \prime}$ & 05431486 & 13.6 & .5660 & relation & 11.6 & 11.6 \\
\hline 147 & 05548170 & N Br Nippersink Ck at Genoa City & $42^{\circ} 29^{\prime} 51^{\prime \prime}$ & $88^{\circ} 20^{\prime} 00^{\prime \prime}$ & 05431486 & 41.7 & .3237 & equation & 22.7 & 7.4 \\
\hline
\end{tabular}

${ }^{1}$ Low-flow discharge measurements at partial-record stations were compared to one or more nearby USGS gaging stations to establish a relation between measured discharge and concurrent daily discharge. The nearby gaging station that provided the best relation with the partial-record station was chosen as the index station and was associated with nearby miscellaneous measurement sites.

${ }^{2}$ Mismatch between surface-water and ground-water drainage area. 

Publishing support provided by the U.S. Geological Survey Science Publishing Network, Columbus Publishing Service Center

For more information concerning the research in this report, contact the

Director, Wisconsin Water Science Center

U.S. Geological Survey

8505 Research Way

Middleton, Wisconsin 53562

http://wi.water.usgs.gov/ 

